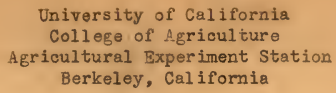

\title{
DECIDUOUS FRUIT STATISTICS \\ as of January, 1942
}

by

\section{S. W. Shear}

\author{
LIBRARY \\ UNIVERSITY OF CALIFORNIA \\ DAVIS \\ Contribution from the \\ Giannini Foundation of Agricultural Economics \\ Wimeographed Report No. 79 \\ UNIVERSITY OF CALIFUKNIA \\ LIBRARY \\ OOULEE OF $\triangle$.RICULTURE \\ ir is
}





\section{DECIDUOUS FRUIT STATISTICS}

as of January, 1942

CONT ENTS

Page

Guide to users $\ldots \ldots \ldots \ldots \ldots \ldots \ldots \ldots \ldots \ldots \ldots \ldots \ldots \ldots$ I

Comparative summaries $\ldots \ldots \ldots \ldots \ldots \ldots \ldots \ldots \ldots \ldots \ldots \ldots$. 1

Apples $\ldots \ldots \ldots \ldots \ldots \ldots \ldots \ldots \ldots \ldots \ldots \ldots \ldots \ldots \ldots \ldots \ldots \ldots, 35$

Apricots $\ldots \ldots \ldots \ldots \ldots \ldots \ldots \ldots \ldots \ldots \ldots \ldots \ldots \ldots \ldots \ldots . . \ldots 42$

Cherries $\ldots \ldots \ldots \ldots \ldots \ldots \ldots \ldots \ldots \ldots \ldots \ldots \ldots \ldots \ldots \ldots, 48$

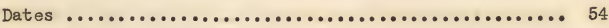

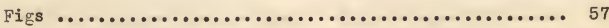

Grapes $\ldots \ldots \ldots \ldots \ldots \ldots \ldots \ldots \ldots \ldots \ldots \ldots \ldots \ldots \ldots \ldots . \ldots \ldots$

Peaches $\ldots \ldots \ldots \ldots \ldots \ldots \ldots \ldots \ldots \ldots \ldots \ldots \ldots \ldots \ldots \ldots \ldots . . \ldots 4$

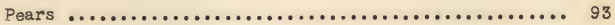

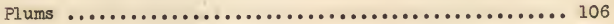

Prunes, dried .............................. 110

NOTE:

LATEST 1941 CALIFORIA DRIGD FRUIT PRODUCTIUIN FIGU KES as reported by the Dried Fruit Association, itarch 13, 1942, are given in Comparative Summaries, table 16 , page 16. Please substitute these for the rough preiminary trade estimates as of January, 1942, on dried fruit production given elsewhere in the tables on Apples, Apricots, Pigs, Peaches, Jears, and Prumes. 


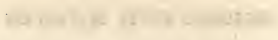

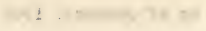
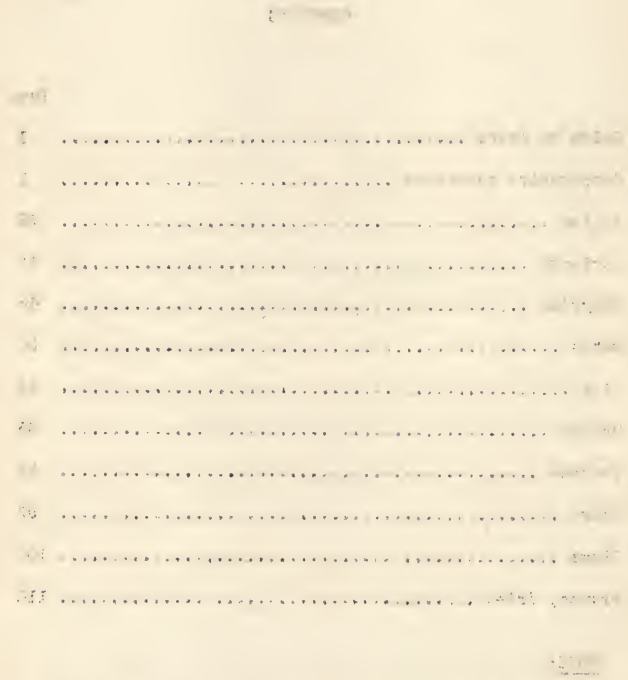

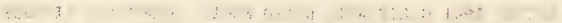

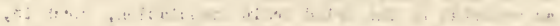

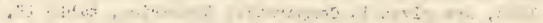

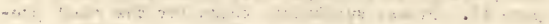
$+\ldots \ldots+\ldots, \ldots, \ldots=$

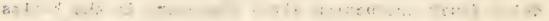

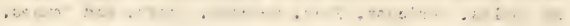




\section{DECIDUOUS FRUIT STATISTICS $1 /$}

\section{as of January, 1942 \\ Compiled by S. T?. Shear $\underline{2 /}$}

\section{GUIDE TO USERS}

Those seriously interested in using this compendium of deciduous fruit statistics will find it helpful to read the following brief comments about the data included and where additional supplementary data may be secured. Users will also find it worth while to read the footnotes to individual tables which briefly describe the data and indicate the original sources.

This is the fourth compendium of selected Deciduous Fruit Statistics published as a special mimeographed report by the Giamini Foundation. The data included have been selected as being the most generally useful of a much larger group of statistics that the compiler has found necessary to keep up to date in order quickly to help responsible groups in diagnosing some of the major economic problems of the deciduous fruit industri es of the state.

This compendium makes readily available, in one place, selected historical series of statistical data on supply, demand, and prices of fruits, evaluated and briefly described, and conveniently arranged to throw light on the direction or tendencies of the more important economic changes in the California deciduous fruit industries. It contains tables relating mostly to ten fruits grown extensively in California, namely, apples, apricots, cherries, dates, figs, grapes, peaches, pears, plums, and dried prunes. However, some data on all fruits, including citrus, are included in the first section, Comparative Sumaries. A section on dates is also included although they are not a deciduous fruit. The

1) Contribution from the Giannini Foundation of Agricultural Economics, Mimeographed Report I'o. 79. January, 1942.

2) Associate Agricultural Economist in the Fxperiment Station and on the Giannin $\bar{i}$ Foundation of Agricultural Economics, University of California. 


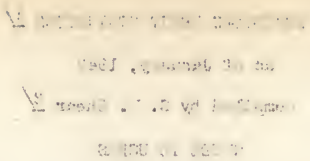

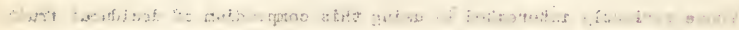

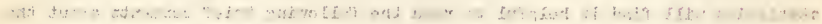

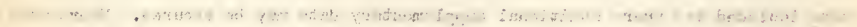

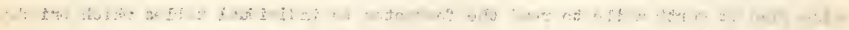

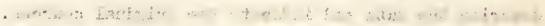

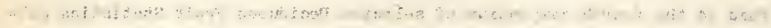

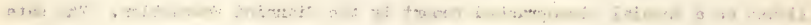

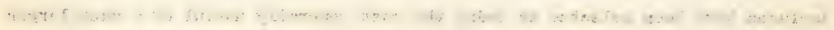

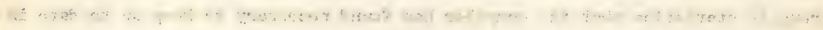

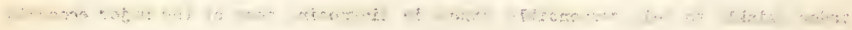

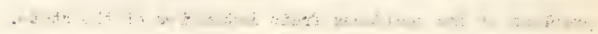

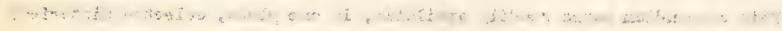

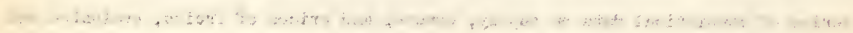

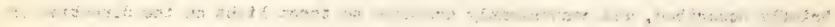

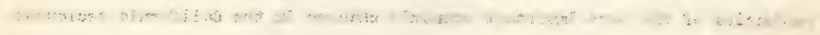

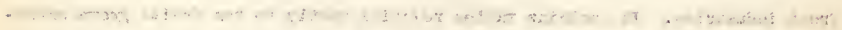

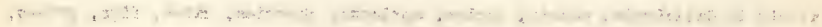

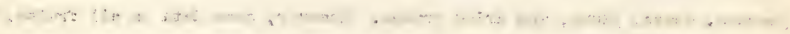

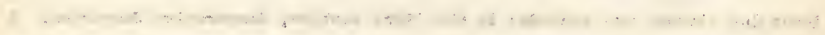

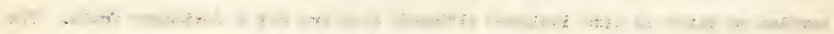

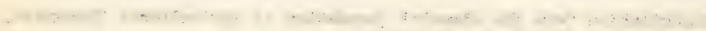

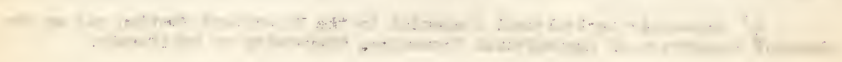


data given are mostly for the past fifteen or twenty crop years and deal largely with acreage, production, yields, utilization, consumption, shipments, exports, imports, and prices, with special reference to California and the United States. Several tables on exports are included to help visualize the importance of our fruit exports to Europe and the extent to which they have been curtailed by the present war. Data on United States exports by country of destination for the crop year, 1940-41, are incomplete as publication was discontinued after March 31, 1941. Publication of all export and import data by individual commodities was also discontinued after September 30, 1941. Some export tables are in the Comparative Summaries and others in the sections on individual fruits. The comparisons of United States and California fruit production with total exports and with exports to Europe by kinds of fruit and by uses, shown in the section on Comparative Summaries, tables 25-28, pages 25-28, are particularly helpful in indicating the relative importance of exports to the fruit industry as a whole and to each fruit during the pre-War period, 1934-1938.

Data on California grape production and utilization in the Comparative Summaries are preliminary official estimates of December, 1941, whereas those in the section on Grapes are unofficial revisions involving data released in March, 1942 , on the commercial crush by the wine Institute and the dried raisin pack by the Dried Fruit Association.

This compendium supplements the extensive body of fruit statistics included in Agricultural Statistios, the statistical yearbook of the United States Department of Agriculture. Of the numerous current reports of the United States Department of Agriculture dealing with fruit statistics, the mimeographed monthly Fruit Situation is of the most general interest. The September, 1941, issue of the Fruit Situation and the October, 1941, is sue of the Agricultural Situation contain very brief 1942 outlook statements and charts on fruits. More detailed statistics are available in the monthly Fruit and Nut Crop Reports of the Federal and State Crop 


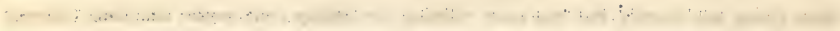

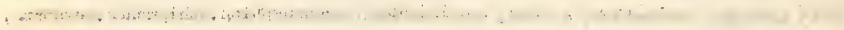

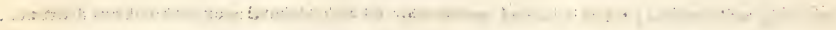

S.

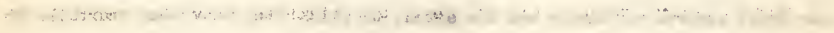

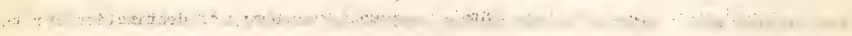

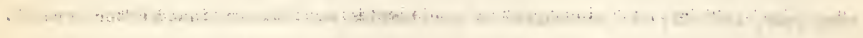

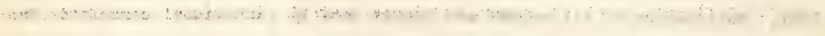

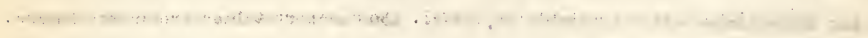

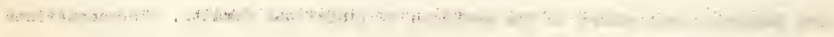

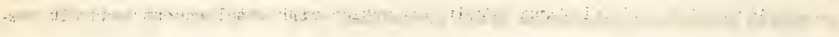

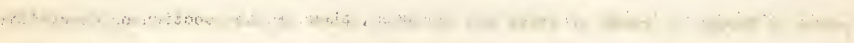

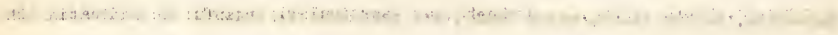

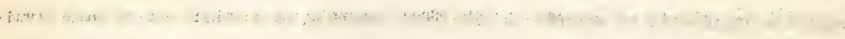

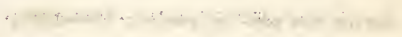

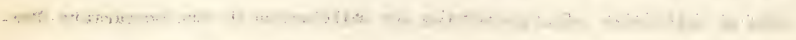

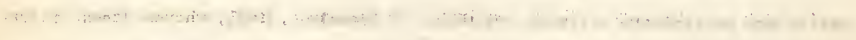

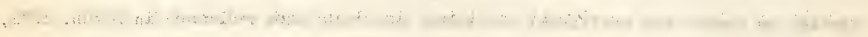

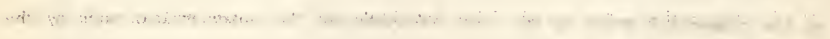

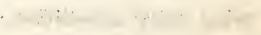

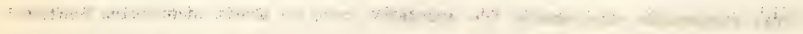

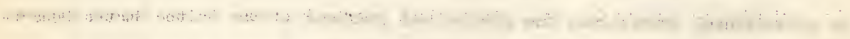

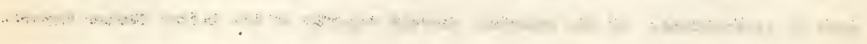

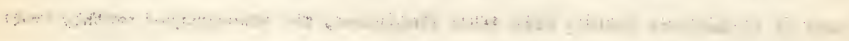

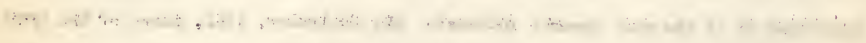

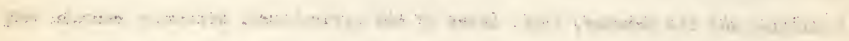

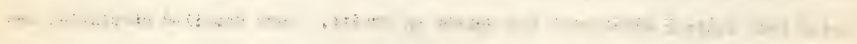

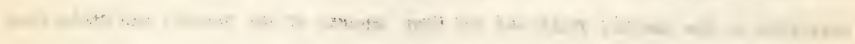


Reporting Service and the numerous releases and annual summaries on fresh and dried fruits of the Federal-State Narket News Service at San Francisco and Sacramento.

Footnotes on sources and description of data as given in the tables are shortened considerably in order to confine each table to one page. Exact details are available in the compiler's files. However, the agency or agencies from whom the compiler secured the data are given credit for their contributions in the source stated at the foot of each table. Those who quote or reproduce data from this report will help to maintain more adequate and improved basic statistics if they, likewise, will give proper credit to the specific agency or agencies named as the original source from which the data have been secured.

Although the compiler takes the responsibility for the tables as presented, this compendium is really the joint contribution of many persons and agencies, official and private, engaged in the collection and compilation of economic statistics. The compiler wishes again to thank each of the persons and agencies who have generously cooperated in making the data in this report available. He is particularly indebted to ir. R. F. Blair of the California Cooperative Crop Reporting Service for his very generous personal assistance. Miss Valerie $\pi$. Smith, Statistical Clerk in the Statistical Laboratory of the Giannini Foundation, deserves much credit for the accuracy of the tables and footnotes which have involved a tremendous amount of detailed, painstaking, thoughtful work in compiling, checking, and documentation. 

University of California, college of Agrioulture

Agricultural Experiment Station, Berkeley, January 1942

DECIDUOUS FRUIT STATISTICS

COIPARATIVE SUMAARIES

Table 1

United States Per-Capita Consumption of Fruits, All Uses,* Averages 1924-1938

\begin{tabular}{|c|c|c|c|c|c|c|c|c|}
\hline \multirow[t]{2}{*}{ Kind of fruit } & \multicolumn{3}{|c|}{ Quantity * } & \multicolumn{3}{|c|}{ Per cent of total } & \multicolumn{2}{|c|}{$\begin{array}{c}\text { Per cent in- } \\
\text { crease or de- } \\
\text { crease 1934- } \\
1938 \text { productiono }\end{array}$} \\
\hline & $\begin{array}{l}1924= \\
1928\end{array}$ & $\begin{array}{l}1929- \\
1933\end{array}$ & $\begin{array}{l}1934- \\
1938\end{array}$ & $\begin{array}{l}1924- \\
1928\end{array}$ & $\begin{array}{l}1929- \\
1933\end{array}$ & $\begin{array}{l}1934- \\
1938\end{array}$ & $\begin{array}{l}1924- \\
1928 \\
\end{array}$ & $\begin{array}{l}1929- \\
1933 \\
\end{array}$ \\
\hline & \multicolumn{3}{|c|}{ Pounds, fresh weight } & \multicolumn{3}{|c|}{ Per cont } & \multicolumn{2}{|c|}{ Per cent } \\
\hline $\begin{array}{l}\text { Total } \\
\text { Deciduous-tree } \\
\text { Grapes } \\
\text { Citrus } \\
\text { Others }\end{array}$ & $\begin{array}{r}198.1 \\
.98 .0 \\
33.8 \\
34.9 \\
31.4\end{array}$ & $\begin{array}{r}184.5 \\
88.1 \\
26.7 \\
40.5 \\
29.2\end{array}$ & $\begin{array}{r}209.3 \\
88.3 \\
30.4 \\
56.7 \\
33.9\end{array}$ & $\begin{array}{r}100.0 \\
49.5 \\
17.1 \\
17.6 \\
15.8\end{array}$ & $\begin{array}{r}100.0 \\
47.7 \\
14.5 \\
22.0 \\
15.8\end{array}$ & $\begin{array}{r}100.0 \\
42.2 \\
14.5 \\
27.1 \\
16.2\end{array}$ & $\begin{array}{l}+6 \\
-10 \\
-10 \\
+62 \\
+8\end{array}$ & $\begin{array}{r}+13 \\
+ \\
+14 \\
+40 \\
+16\end{array}$ \\
\hline $\begin{array}{l}\text { Deciduous-tree, } \\
\text { total } \\
\text { Apples } \\
\text { Peaches } \\
\text { Pears } \\
\text { Prunes } \\
\text { Apricots } \\
\text { Flums } \\
\text { Cherries } \\
\text { Figs }\end{array}$ & $\begin{array}{r}98.0 \\
59.5 \\
20.3 \\
7.3 \\
4.2 \\
1.8 \\
1.8 \\
1.6 \\
1.5\end{array}$ & $\begin{array}{r}88.1 \\
52.3 \\
17.0 \\
7.0 \\
4.1 \\
2.4 \\
2.0 \\
2.0 \\
1.3\end{array}$ & $\begin{array}{r}88.3 \\
49.7 \\
17.9 \\
8.4 \\
4.8 \\
2.0 \\
2.0 \\
2.0 \\
1.5\end{array}$ & $\begin{array}{r}49.5 \\
30.0 \\
10.3 \\
3.7 \\
2.1 \\
0.9 \\
0.9 \\
0.8 \\
0.8\end{array}$ & $\begin{array}{r}47.7 \\
28.3 \\
9.2 \\
3.8 \\
2.2 \\
1.3 \\
1.1 \\
1.1 \\
0.7\end{array}$ & $\begin{array}{r}42.2 \\
23.7 \\
8.5 \\
4.0 \\
2.3 \\
1.0 \\
1.0 \\
1.0 \\
0.7\end{array}$ & $\begin{array}{r}-10 \\
-16 \\
-12 \\
+15 \\
+14 \\
+11 \\
+11 \\
+25 \\
0\end{array}$ & $\begin{array}{r}+ \\
-5 \\
+5 \\
+20 \\
+17 \\
-17 \\
0 \\
0 \\
+15\end{array}$ \\
\hline Grapes, total & 33.8 & $26 \cdot 7$ & 30.4 & $17 \cdot 1$ & 14.5 & $14 \cdot 5$ & -10 & +14 \\
\hline $\begin{array}{l}\text { Citrus, total } \\
\text { Oranges } \\
\text { Grapefruit } \\
\text { Lemons }\end{array}$ & $\begin{array}{r}34.9 \\
23.2 \\
7.1 \\
4.6\end{array}$ & $\begin{array}{r}40.5 \\
27.2 \\
8.9 \\
4.4\end{array}$ & $\begin{array}{r}56.7 \\
35.5 \\
16.0 \\
5.2\end{array}$ & $\begin{array}{r}17.6 \\
11.7 \\
3.6 \\
2.3\end{array}$ & $\begin{array}{r}22.0 \\
14.8 \\
4.8 \\
2.4\end{array}$ & $\begin{array}{r}27.1 \\
17.0 \\
7.6 \\
2.5\end{array}$ & $\begin{array}{r}+62 \\
+53 \\
+125 \\
+13\end{array}$ & $\begin{array}{l}+40 \\
+31 \\
+80 \\
+18\end{array}$ \\
\hline $\begin{array}{l}\text { Others, total } \\
\text { Dates } \\
\text { Cranberries } \\
\text { Strawberries } \\
\text { Bananas } \\
\text { Pineapple }\end{array}$ & $\begin{array}{r}31.4 \\
1.8 \\
0.5 \\
3.4 \\
20.3 \\
5.4\end{array}$ & $\begin{array}{r}29.2 \\
1.6 \\
0.5 \\
3.2 \\
18.0 \\
5.9\end{array}$ & $\begin{array}{r}33.9 \\
1.9 \\
0.4 \\
3.0 \\
19.5 \\
9.1\end{array}$ & $\begin{array}{r}15.8 \\
0.9 \\
0.3 \\
1.7 \\
10.2 \\
2.7\end{array}$ & $\begin{array}{r}15.8 \\
0.9 \\
0.3 \\
1.7 \\
9.7 \\
3.2\end{array}$ & $\begin{array}{r}16.2 \\
0.9 \\
0.2 \\
1.4 \\
9.3 \\
4.4\end{array}$ & $\begin{array}{l}+8 \\
+6 \\
-20 \\
-12 \\
-4 \\
+69\end{array}$ & $\begin{array}{l}+16 \\
+19 \\
-20 \\
-6 \\
+8 \\
+54\end{array}$ \\
\hline
\end{tabular}

* Approximate quantity of fresh fruit as harvested with no deductions for loss of weight in commercial processing and packing or in marketing and in

home preparation for consumption. Includes consumption of cenner? fruit salad and ccoktail and oried frit salad.

† Loss th.an + 0.5\%.

Source of data: Complled by $S$. W. Shear, Giannini Foundation of Agricultural Economics, University of California, largely upon the basis of offioial data. 

Table 2. United States Per-Capita Consumption* of Dried Fruits and of All Uses of These Fruits, in Terms of Fresh and of Dry Weight, Averages 1924-1938

\begin{tabular}{|c|c|c|c|c|c|c|c|c|c|c|c|c|}
\hline \multirow{4}{*}{$\begin{array}{l}\text { Kind } \\
\text { of } \\
\text { fruit }\end{array}$} & \multicolumn{4}{|c|}{ 1924-1928 } & \multicolumn{4}{|c|}{ 1929-1933 } & \multicolumn{4}{|c|}{$1934-1938$} \\
\hline & \multirow{3}{*}{$\begin{array}{c}\text { Total } \\
\text { all uses, } \\
\text { fresh } \\
\text { weight }\end{array}$} & \multirow{3}{*}{$\begin{array}{c}\text { Dr } \\
\text { Per cent } \\
\text { of } \\
\text { total }\end{array}$} & ied & & \multirow{3}{*}{$\begin{array}{l}\text { Total } \\
\text { all uses, } \\
\text { fresh } \\
\text { weight }\end{array}$} & \multicolumn{3}{|c|}{ Dried } & \multirow{3}{*}{$\begin{array}{c}\text { Total } \\
\text { all uses, } \\
\text { fresh } \\
\text { weight }\end{array}$} & \multicolumn{3}{|c|}{ Dried } \\
\hline & & & Quent & ity & & \multirow{2}{*}{$\begin{array}{l}\text { Per cent } \\
\text { of } \\
\text { total }\end{array}$} & \multicolumn{2}{|c|}{ Quantity } & & \multirow{2}{*}{$\begin{array}{c}\text { Per cent } \\
\text { of } \\
\text { total }\end{array}$} & \multicolumn{2}{|c|}{ Quantity } \\
\hline & & & $\begin{array}{l}\text { Fresh } t \\
\text { weight }\end{array}$ & $\begin{array}{c}\text { Dry } \\
\text { weight }\end{array}$ & & & $\begin{array}{l}\text { Fresh } \\
\text { weight }\end{array}$ & $\begin{array}{c}\text { Dry } \\
\text { weight }\end{array}$ & & & $\begin{array}{l}\text { Fresh }+ \\
\text { weight }\end{array}$ & $\begin{array}{c}\text { Dry } \\
\text { weight }\end{array}$ \\
\hline & Pounds & Per cent & \multicolumn{3}{|c|}{ Pounds } & Per cent & \multicolumn{3}{|c|}{ Pounds } & Per cent & \multicolumn{2}{|c|}{ Founds } \\
\hline $\begin{array}{l}\text { Total } \\
\text { Deciduous }\end{array}$ & 130.2 & 18.0 & 23.5 & 6.34 & 112.4 & 18.1 & 20.3 & 5.49 & 116.6 & 18.0 & 21.0 & 5.76 \\
\hline tree $\neq$ & 94.6 & 10.1 & 9.6 & 2.85 & 84.1 & 10.9 & 9.2 & 2.72 & 84.3 & 11.7 & 9.9 & 2.99 \\
\hline Grapes & 33.8 & 35.8 & 12.1 & 3.03 & 26.7 & 35.6 & 9.5 & 2.37 & 30.4 & 30.3 & 9.2 & 2.30 \\
\hline Dates & 1.8 & 100.0 & 1.8 & 0.46 & 1.6 & 100.0 & 1.6 & 0.40 & 1.9 & 100.0 & 1.9 & 0.47 \\
\hline Deciduous & & & & & & & & & & & & \\
\hline tree $\neq$ & 94.6 & 10.1 & 9.6 & 2.85 & 84.1 & 10.9 & 9.2 & 2.72 & 84.3 & 11.7 & 9.9 & 2.99 \\
\hline Apples & 59.5 & 1.8 & 1.1 & 0.16 & 52.3 & 1.5 & 0.8 & 0.12 & 49.7 & 1.8 & 0.9 & 0.13 \\
\hline Peaches & 20.3 & 8.9 & 1.8 & 0.33 & 17.0 & 8.8 & 1.5 & 0.27 & 17.9 & 8.9 & 1.6 & 0.29 \\
\hline Pears & 7.3 & 1.4 & 0.1 & 0.02 & 7.0 & 1.4 & 0.1 & 0.01 & 8.4 & 1.2 & 0.1 & 0.02 \\
\hline Prunes & 4.2 & 100.0 & 4.2 & 1.70 & 4.1 & 100.0 & 4.1 & 1.66 & 4.8 & 100.0 & 4.8 & 1.90 \\
\hline Aprictos & 1.8 & 55.6 & 1.0 & 0.18 & 2.4 & 62.5 & 1.5 & 0.27 & 2.0 & 60.0 & 1.2 & 0.21 \\
\hline Figs $\oint$ & 1.5 & 93.3 & 1.4 & 0.46 & 1.3 & 92.3 & 1.2 & 0.39 & 1.5 & 86.7 & 1.3 & 0.44 \\
\hline
\end{tabular}

* Consumption of fruits in dried fruit salad or compote is included in data by kind of fruit.

T Unprocessed dry weight converted to fresh equivalent by multiplying by the following factors: apples, 7 ; peaches, pears, and apricots, 5.5; prunes, 2.5; figs, 3 ; and raisins and dates, 4.

$\neq$ Includes only fruits listed in this table. $\quad \zeta$ Includes all grades of figs, standard and substandard.

Sources of data:

Compiled by S. W. Shear, Giannini Foundation of Agricultural Economics, University of California, from official data and reliable unofficial trade estimates. 


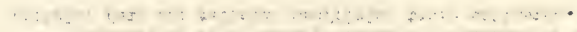

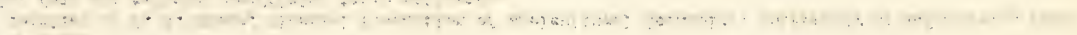

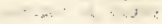

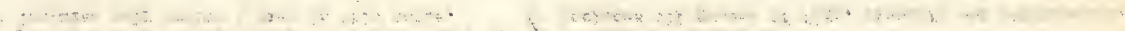

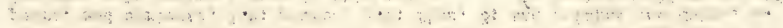

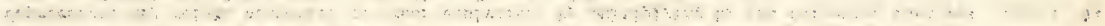

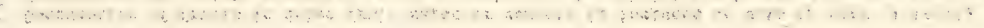

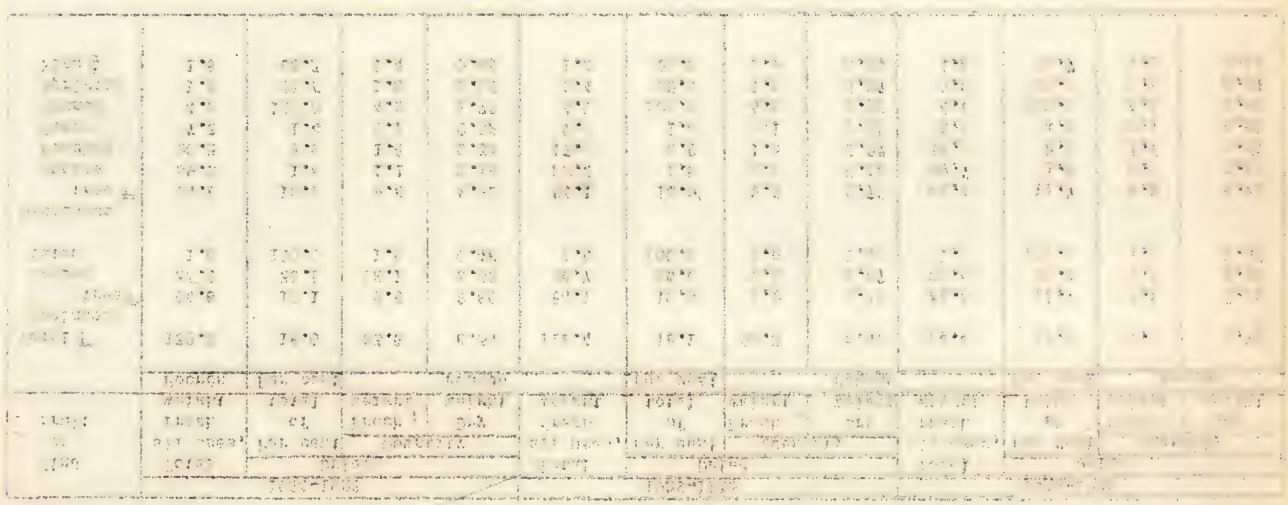

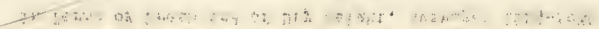

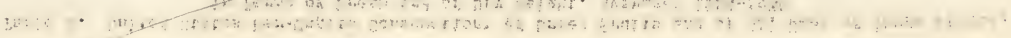

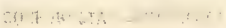


Table 3. United States Per-Capita Consumption* of Chief ${ }^{\dagger}$ Canned Fruits ${ }^{\ddagger}$ and of All Uses of These Fruits in Terms of Fresh Weight, Averages 1924-1938

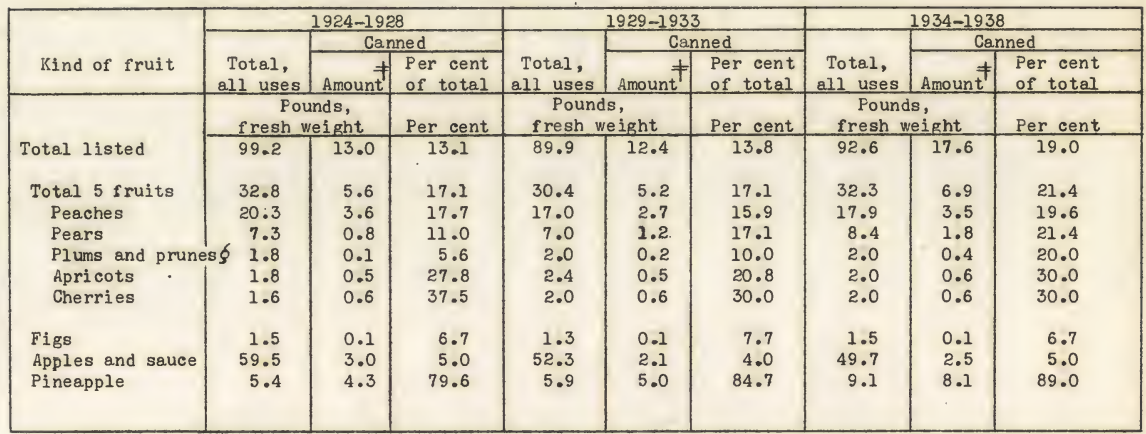

* Consumption of fruits in canned fruit salad and cocktail is included in data by kind of fruit.

+ Table excludes canned citrus as complete data unavailable except 1934-1938 average of 6.4 pounds.

\# Approximate quantity of fresh fruit used in canning including loss in weight from peeling, coring, and pitting.

$\oint$ Includes only canned fresh prunes.

Sources of data:

Compiled by S. W. Shear, Giannini Foundation of Agricultural Economics, University of California, from official data and reliable trade estimates. 


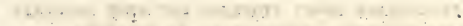

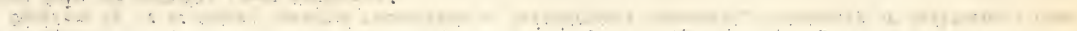
$\therefore \because \div, \cdots+$
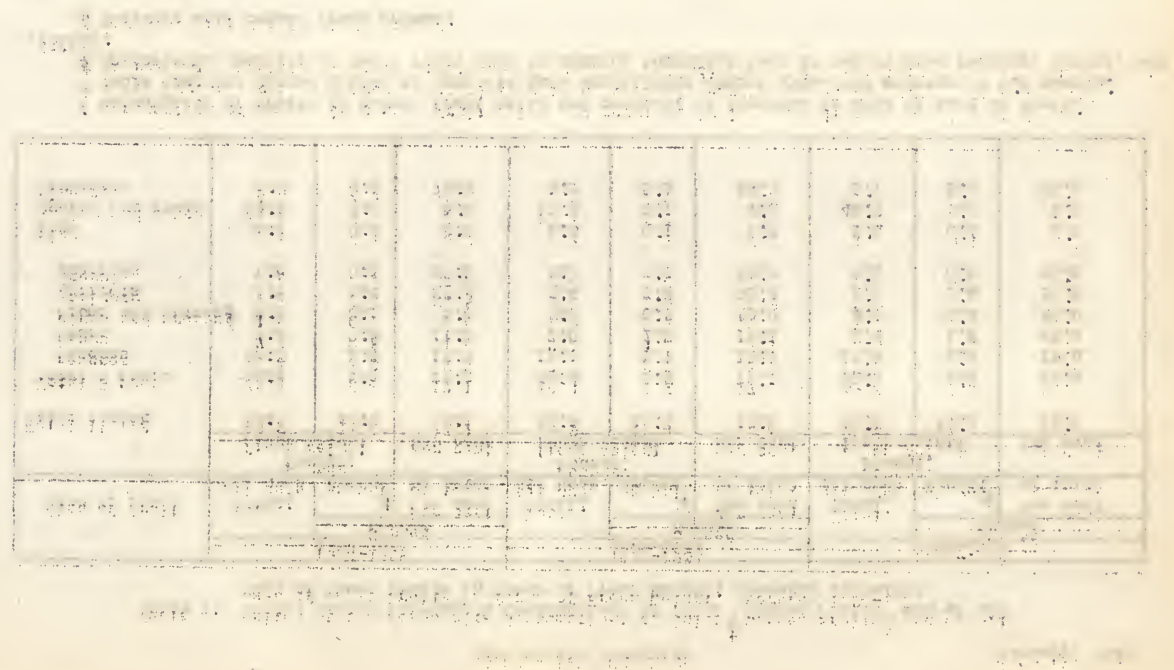
Table 4. United States and California Total Production* of Chief Fruits, Averages 1919-1938

\begin{tabular}{|c|c|c|c|c|c|c|c|c|c|c|c|c|}
\hline \multirow[b]{3}{*}{ Kind of fruit } & \multicolumn{3}{|c|}{$1919-1923$} & \multicolumn{3}{|c|}{$1924-1928$} & \multicolumn{3}{|c|}{$1929-1933$} & \multicolumn{3}{|c|}{$1934-1938$} \\
\hline & \multirow[b]{2}{*}{$\begin{array}{c}\text { United } \\
\text { States } \\
\text { produc- } \\
\text { tion }\end{array}$} & \multicolumn{2}{|c|}{ California } & \multirow[b]{2}{*}{$\begin{array}{l}\text { United } \\
\text { States } \\
\text { produc- } \\
\text { tion }\end{array}$} & \multicolumn{2}{|c|}{ Californis } & \multirow[b]{2}{*}{$\begin{array}{l}\text { United } \\
\text { States } \\
\text { produc } \\
\text { tion }\end{array}$} & \multicolumn{2}{|c|}{ California } & \multirow[b]{2}{*}{$\begin{array}{c}\text { United } \\
\text { States } \\
\text { produc- } \\
\text { tion }\end{array}$} & \multicolumn{2}{|c|}{ Californis } \\
\hline & & Quaintity & $\begin{array}{c}\text { Per cent } \\
\text { of } \\
\text { United } \\
\text { States }\end{array}$ & & Guantity & $\begin{array}{c}\text { Per cent } \\
\text { of } \\
\text { United } \\
\text { States }\end{array}$ & & Quantity & \begin{tabular}{|c|} 
Per cent \\
of \\
United \\
States \\
\end{tabular} & & Quantity & $\begin{array}{l}\text { Per cent } \\
\text { of } \\
\text { United } \\
\text { States }\end{array}$ \\
\hline & $\begin{array}{r}1,000 \mathrm{~s} \\
\text { fres }\end{array}$ & $\begin{array}{l}\text { short tons } \\
\text { h wt. }\end{array}$ & fer cent & $\begin{array}{l}1,000 \mathrm{sh} \\
\text { fres }\end{array}$ & $\begin{array}{l}\text { nort tons } \\
\text { n wt. }\end{array}$ & Per cent & $\begin{array}{r}1,000 \mathrm{sh} \\
\text { fresh }\end{array}$ & $\begin{array}{l}\text { nort tons } \\
\text { vt. }\end{array}$ & Per cent & $\begin{array}{r}1,000 \mathrm{sh} \\
\text { fresh }\end{array}$ & $\begin{array}{l}\text { hort } t \\
\text { wt. }\end{array}$ & Per cent \\
\hline $\begin{array}{l}\text { Total listed } \\
\text { Deciduous tree } \\
\text { Grapes } \\
\text { Citrus }\end{array}$ & \begin{tabular}{|l|}
9,511 \\
6,136 \\
1,730 \\
1,645
\end{tabular} & $\begin{array}{r}3,637 \\
1,228 \\
1,511 \\
898\end{array}$ & $\begin{array}{l}38.2 \\
20.0 \\
87.3 \\
54.6\end{array}$ & $\begin{array}{r}11,222 \\
6,776 \\
2,333 \\
2,113\end{array}$ & $\begin{array}{l}4,894 \\
1,600 \\
2,097 \\
1,197\end{array}$ & $\begin{array}{l}43.6 \\
23.6 \\
89.9 \\
56.6\end{array}$ & $\begin{array}{r}11,570 \\
6,837 \\
2,071 \\
2,662\end{array}$ & $\begin{array}{l}5,046 \\
1,874 \\
1,783 \\
1,389\end{array}$ & \begin{tabular}{|l|}
43.6 \\
27.4 \\
86.1 \\
52.2
\end{tabular} & $\begin{array}{r}13,212 \\
6,851 \\
2,368 \\
3,993\end{array}$ & $\begin{array}{l}5,780 \\
1,884 \\
2,118 \\
1,778\end{array}$ & $\begin{array}{l}43.7 \\
27.5 \\
89.4 \\
44.5\end{array}$ \\
\hline $\begin{array}{l}\text { Deciduous tree } \\
\text { Apples } \\
\text { Peaches } \\
\text { Pears } \\
\text { Prunes, dried } \\
\text { Apricots* } \\
\text { Plums* } \\
\text { Cherries } \\
\text { Figs } \neq\end{array}$ & $\begin{array}{c}6,136 \\
3,904 \\
1,116 \\
403 \\
369 \\
150+ \\
91 \\
68 \\
35+1\end{array}$ & $\begin{array}{r}1,228 \\
193 \\
380 \\
118 \\
289 \\
150 \\
47 \\
16 \\
35\end{array}$ & $\begin{array}{c}20.0 \\
4.9 \\
34.1 \\
29.3 \\
78.3 \\
100.0+ \\
51.6 \\
23.5 \\
100.0+\end{array}$ & $\begin{array}{r}6,776 \\
4,013 \\
1,315 \\
523 \\
521 \\
171 \\
110 \\
83 \\
40\end{array}$ & $\begin{array}{r}1,600 \\
220 \\
476 \\
185 \\
441 \\
170 \\
57 \\
15 \\
36\end{array}$ & $\begin{array}{r}23.6 \\
5.5 \\
36.2 \\
35.4 \\
84.6 \\
99.4 \\
51.8 \\
18.1 \\
90.0\end{array}$ & $\begin{array}{r}6,837 \\
3,805 \\
1,275 \\
598 \\
584 \\
250 \\
134 \\
125 \\
66\end{array}$ & $\begin{array}{r}1,874 \\
226 \\
555 \\
229 \\
472 \\
246 \\
62 \\
20 \\
64\end{array}$ & $\begin{array}{r}27.4 \\
5.9 \\
43.5 \\
38.3 \\
80.8 \\
98.4 \\
46.3 \\
16.0 \\
97.0\end{array}$ & $\begin{array}{r}6,851 \\
3,669 \\
1,255 \\
700 \\
643 \\
225 \\
136 \\
134 \\
89\end{array}$ & $\begin{array}{r}1,884 \\
210 \\
497 \\
229 \\
563 \\
216 \\
61 \\
21 \\
87\end{array}$ & $\begin{array}{r}27.5 \\
5.7 \\
39.6 \\
32.7 \\
87.6 \\
96.0 \\
44.9 \\
15.7 \\
97.8\end{array}$ \\
\hline $\begin{array}{l}\text { Grapes, total } \\
\text { Raisin varietie } \\
\text { "ine varieties } \\
\text { Tuble varieties }\end{array}$ & $\begin{array}{r}1,730 \\
887 \\
399 \\
444\end{array}$ & $\begin{array}{r}1,513 \\
887 \\
399 \\
225\end{array}$ & $\begin{array}{r}87.3 \\
100.0 \\
100.0 \\
50.7\end{array}$ & $\begin{array}{r}2,333 \\
1,248 \\
423 \\
662\end{array}$ & $\begin{array}{r}2,097 \\
1,248 \\
423 \\
426\end{array}$ & $\begin{array}{r}89.9 \\
100.0 \\
100.0 \\
64.4\end{array}$ & $\begin{array}{r}2,071 \\
1,074 \\
405 \\
592\end{array}$ & $\begin{array}{r}1,783 \\
1,074 \\
405 \\
304\end{array}$ & $\begin{array}{r}86.1 \\
100.0 \\
100.0 \\
51.4\end{array}$ & $\begin{array}{r}2,368 \\
1,189 \\
558 \\
621\end{array}$ & $\begin{array}{r}2,118 \\
1,189 \\
558 \\
371\end{array}$ & $\begin{array}{r}89.4 \\
100.0 \\
100.0 \\
59.7\end{array}$ \\
\hline $\begin{array}{l}\text { Citrus, total } \\
\text { Oranges } \\
\text { Grapefruit } \\
\text { Lemons }\end{array}$ & $\begin{array}{r}1,645 \\
1,165 \\
292 \\
188\end{array}$ & $\begin{array}{r}898 \\
699 \\
11 \\
188\end{array}$ & $\begin{array}{r}54.6 \\
60.0 \\
3.8 \\
100.0\end{array}$ & $\begin{array}{r}2,113 \\
1,473 \\
393 \\
247\end{array}$ & $\begin{array}{r}1,197 \\
930 \\
20 \\
247\end{array}$ & $\begin{array}{r}56.6 \\
63.1 \\
5.1 \\
100.0\end{array}$ & $\begin{array}{r}2,662 \\
1,811 \\
579 \\
272\end{array}$ & $\begin{array}{r}1,389 \\
1,076 \\
41 \\
272\end{array}$ & $\begin{array}{r}52.2 \\
59.4 \\
7.1 \\
100.0\end{array}$ & $\begin{array}{r}3,993 \\
2,518 \\
1,121 \\
354\end{array}$ & $\begin{array}{r}1,778 \\
1,365 \\
59 \\
354\end{array}$ & $\begin{array}{r}44.5 \\
54.2 \\
5.3 \\
100.0\end{array}$ \\
\hline
\end{tabular}
available.

+No adequate data sveileble on the small quentities produced in states other than California.

\# Merchantable and nonmerchantable figs. \$ Production in states other than Culifornia included in table varieties. Sources of data: Complled by S. W. Shear, Giannini Foundation, from United States and California Crop Reports. 


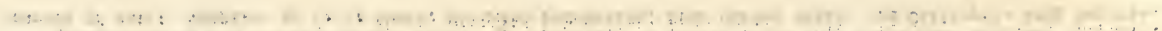
s. A.

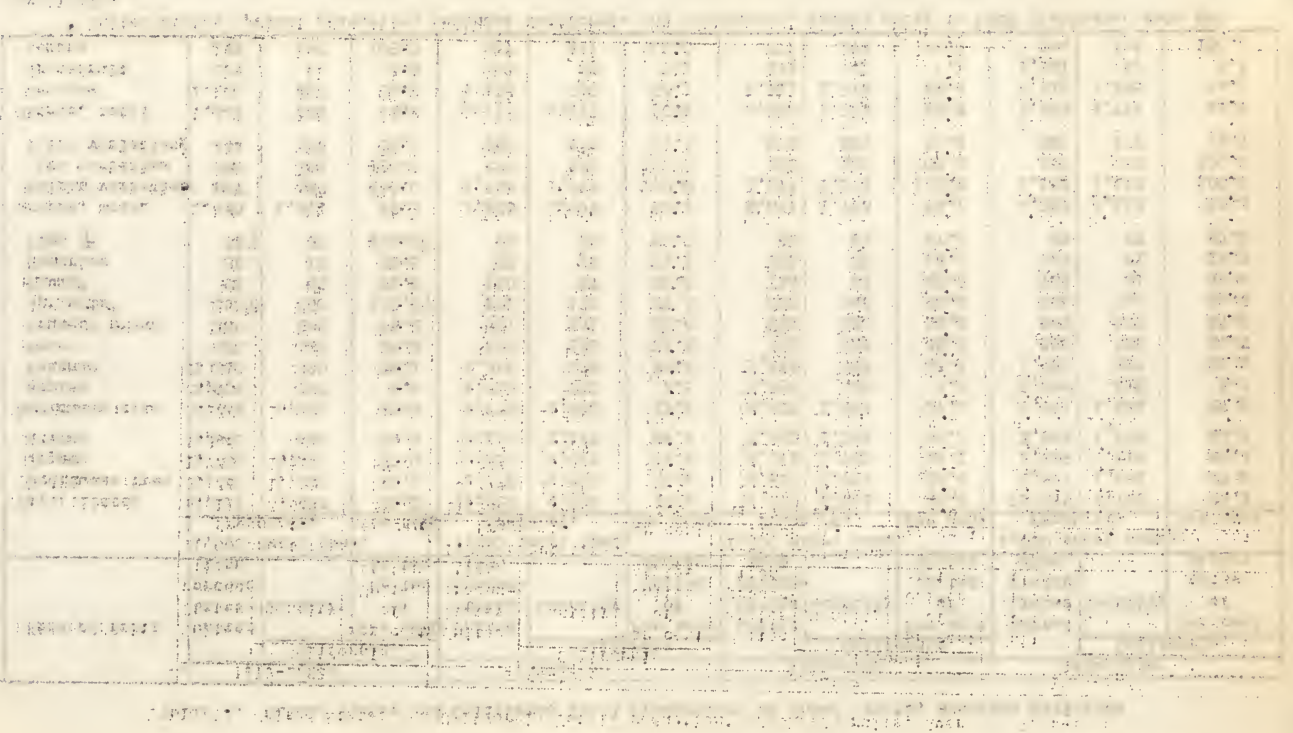

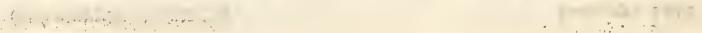


Table 5. United States Fruit Production* by Groups -- Citrus, Grapes, and Deciduous Tree, 1919-1941

\begin{tabular}{|c|c|c|c|c|c|c|}
\hline \multirow{3}{*}{$\begin{array}{l}\text { Crop } \\
\text { yeart }\end{array}$} & \multirow{3}{*}{$\begin{array}{l}\text { Total } \\
\text { deciduous } \\
\text { and citrus }\end{array}$} & \multirow{3}{*}{ Citrus ${ }^{\dagger}$} & \multicolumn{4}{|c|}{ Deciduous fruits } \\
\hline & & & \multirow[b]{2}{*}{ Total } & \multirow[b]{2}{*}{ Grapes } & \multicolumn{2}{|c|}{ Tree fruits } \\
\hline & & & & & Total & $\begin{array}{l}\text { Excluding } \\
\text { apples }\end{array}$ \\
\hline \multirow[b]{3}{*}{ Averages : } & 1 & 2 & 3 & 4 & 5 & 6 \\
\hline & \multicolumn{6}{|c|}{ Short tons, equivalent fresh weight } \\
\hline & & & & & & \\
\hline 1919-1923 & $9,510,600$ & $1,644,600$ & $7,866,000$ & $1,730,200$ & $6,135,800$ & $2,232,000$ \\
\hline $1924-1928$ & $11,222,600$ & $2,113,400$ & $9,109,200$ & $2,333,000$ & $6,776,200$ & $2,763,100$ \\
\hline $1929-1933$ & $11,570,400$ & $2,662,200$ & $8,908,200$ & $2,071,400$ & $6,836,800$ & $3,032,200$ \\
\hline $1934-1938$ & $13,212,200$ & $3,992,700$ & $9,219,500$ & $2,368,600$ & $6,850,900$ & $3,182,400$ \\
\hline \multicolumn{7}{|l|}{ Annual: } \\
\hline 1919 & $8,689,700$ & $1,369,400$ & $7,320,300$ & $1,575,000$ & $5,745,300$ & $2,370,100$ \\
\hline 1920 & $10,303,700$ & $1,723,400$ & $8,580,300$ & $1,521,000$ & $7,059,300$ & $2,098,800$ \\
\hline 1921 & $6,480,500$ & $1,325,500$ & $5,155,000$ & $1,220,000$ & $3,935,000$ & $1,639,700$ \\
\hline 1922 & $11,053,600$ & $1,722,700$ & $9,330,900$ & $2,085,000$ & $7,245,900$ & $2,699,700$ \\
\hline 1923 & $11,025,500$ & $2,081,900$ & $8,945,600$ & $2,250,000$ & $6,693,600$ & $2,351,600$ \\
\hline 1924 & $9,843,500$ & $1,746,800$ & $8,096,700$ & $1,775,000$ & $6,321,700$ & $2,470,700$ \\
\hline 1925 & $10,153,700$ & $1,933,700$ & $8,220,000$ & $2,200,000$ & $6,020,000$ & $2,361,800$ \\
\hline 1926 & $13,294,700$ & $2,143,200$ & $11,151,500$ & $2,444,000$ & $8,707,500$ & $3,195,800$ \\
\hline 1927 & $9,739,400$ & $1,799,000$ & $7,940,400$ & $2,592,000$ & $5,348,400$ & $2,571,400$ \\
\hline 1928 & $13,081,800$ & $2,944,400$ & $10,137,400$ & $2,654,000$ & $7,483,400$ & $3,215,900$ \\
\hline 1929 & $9,787,400$ & $1,886,100$ & $7,901,300$ & $2,085,000$ & $5,816,300$ & $2,574,100$ \\
\hline 1930 & $12,694,100$ & $3,157,300$ & $9,536,800$ & $2,456,000$ & $7,080,800$ & $3,322,000$ \\
\hline 1931 & $13,052,000$ & $2,778,400$ & $10,273,600$ & $1,646,000$ & $8,627,600$ & $3,697,900$ \\
\hline 1932 & $11,344,400$ & $2,813,800$ & $8,530,600$ & $2,231,000$ & $6,299,600$ & $2,775,200$ \\
\hline 1933 & $10,973,800$ & $2,675,300$ & $8,298,500$ & $1,939,000$ & $6,359,500$ & $2,791,700$ \\
\hline 1934 & $11,479,800$ & $3,655,500$ & $7,824,300$ & $1,958,000$ & $5,866,300$ & $2,849,000$ \\
\hline 1935 & $13,057,100$ & $3,001,900$ & $10,055,200$ & $2,488,000$ & $7,567,200$ & $3,297,200$ \\
\hline 1936 & $11,257,100$ & $3,639,100$ & $7,618,000$ & $1,916,000$ & $5,702,000$ & $2,881,900$ \\
\hline 1937 & $15,779,300$ & $4,432,100$ & $11,347,200$ & $2,777,000$ & $8,570,200$ & $3,511,400$ \\
\hline 1938 & $14,487,900$ & $5,234,900$ & $9,253,000$ & $2,704,000$ & $6,549,000$ & $3,372,500$ \\
\hline 1939 & $15,320,200$ & $4,769,700$ & $10,550,500$ & $2,526,000$ & $8,024,500$ & $3,560,500$ \\
\hline 1940 & $14,621,200$ & $5,608,700$ & $9,012,500$ & $2,548,000$ & $6,464,500$ & $3,104,500$ \\
\hline 1941$\}$ & $15,566,600$ & $5,473,600$ & $10,093,000$ & $2,651,000$ & $7,442,000$ & $3,602,000$ \\
\hline & & & & & & \\
\hline
\end{tabular}

* Includes harvested and unharvested production.

+ Citrus data are for 12 months beginnine November 1 of year indicated.

$\neq$ Includes oranges, grapefruit, and lemons; exoludes limes.

g Preliminary.

Sources of data:

Compil ed by S. W. Shear, Giannini Foundation of Agricultural Fconomios, University of California, largely based on data from United States and Cal ifornia Crop Reports. 


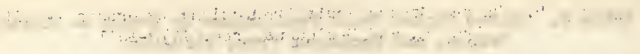

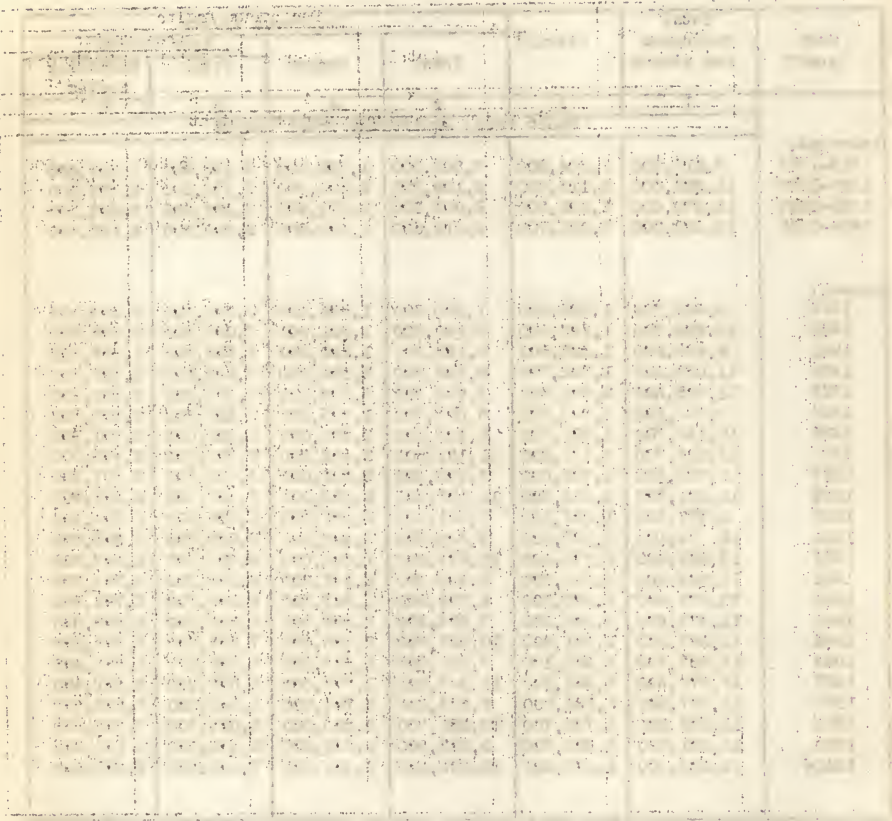

$+4+4=\cdots+\cdots+\cdots+\cdots$

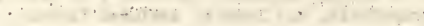


Table 6. United States Production* of Chief Kinds of Deciduous Tree Fruits, 1919-1941

\begin{tabular}{|c|c|c|c|c|c|c|c|c|c|}
\hline Crop & Total & Apples & Apricotst & Cherries & Figs $\neq$ & Peaches & Pears & Plums & Prunes \\
\hline year & 1 & 2 & 3 & 4 & 5 & 6 & 7 & 8 & 9 \\
\hline & \multicolumn{9}{|c|}{ Short tons, equivalent fresh weight } \\
\hline 1919 & $5,745,300$ & $3,375,200$ & 172,000 & $56,300 \oint$ & 36,900 & $1,244,400$ & 367,600 & 77,100 & 415,800 \\
\hline 1920 & $7,059,300$ & $4,960,500$ & 108,000 & $66,800 \oint$ & 38,100 & $1,073,400$ & 431,400 & 69,500 & 311,600 \\
\hline 1921 & $3,935,000$ & $2,295,300$ & 100,000 & 44,900 \& & 30,400 & 793,500 & 285,300 & 87,600 & 298,000 \\
\hline 1922 & $7,245,900$ & $4,546,200$ & 163,000 & $84,100 \oint$ & 35,600 & $1,385,700$ & 505,900 & 87,900 & 437,500 \\
\hline 1923 & $6,693,600$ & $4,342,000$ & 210,000 & 86,700 ई & 32,700 & $1,082,900$ & 426,500 & 131,600 & 381,200 \\
\hline 1924 & $6,321,700$ & $3,851,000$ & 142,000 & 79,300 & 28,900 & $1,248,100$ & 462,600 & 74,800 & 435,000 \\
\hline 1925 & $6,020,000$ & $3,658,200$ & 150,000 & 73,800 & 34,100 & $1,098,400$ & 498,100 & 88,200 & 419,200 \\
\hline 1926 & $8,707,500$ & $5,511,700$ & 176,000 & 107,500 & 44,300 & $1,600,400$ & 612,500 & 132,300 & 522,800 \\
\hline 1927 & $5,348,400$ & $2,777,000$ & 209,600 & 61,400 & 46,300 & $1,044,400$ & 450,700 & 113,200 & 645,800 \\
\hline 1928 & $7,483,400$ & $4,267,500$ & 179,000 & 93,700 & 47,100 & $1,585,400$ & 591,400 & 139,100 & 580,200 \\
\hline 1929 & $5,816,300$ & $3,242,200$ & 221,100 & 98,300 & 61,100 & $1,073,700$ & 532,100 & 130,400 & 457,400 \\
\hline 1930 & $7,080,800$ & $3,758,800$ & 203,400 & 121,800 & 73,700 & $1,328,200$ & 664,100 & 161,100 & 769,700 \\
\hline 1931 & $8,627,600$ & $4,929,700$ & 282,600 & 126,300 & 59,200 & $1,849,200$ & 617,000 & 129,200 & 634,400 \\
\hline 1932 & $6,299,600$ & $3,524,400$ & 274,800 & 143,300 & 64,000 & $1,039,000$ & 595,400 & 135,900 & 522,800 \\
\hline 1933 & $6,359,500$ & $3,567,800$ & 270,300 & 133,800 & 71,100 & $1,086,500$ & 578,900 & 113,900 & 537,200 \\
\hline 1934 & $5,866,300$ & $3,017,300$ & 148,500 & 131,200 & 80,500 & $1,144,400$ & 676,200 & 135,400 & 532,800 \\
\hline 1935 & $7,567,200$ & $4,270,000$ & 223,300 & 138,000 & 83,800 & $1,312,500$ & 625,700 & 131,300 & 782,600 \\
\hline 1936 & $5,702,000$ & $2,820,100$ & 254,000 & 115,200 & 72,500 & $1,139,600$ & 669,100 & 145,500 & 486,000 \\
\hline 1937 & $8,570,200$ & $5,058,800$ & 321,100 & 144,700 & 99,700 & $1,433,300$ & 729,400 & 137,200 & 646,000 \\
\hline 1938 & $6,549,000$ & $3,176,500$ & 178,000 & 140,900 & 106,700 & $1,246,700$ & 800,000 & 130,200 & 770,000 \\
\hline 1939 & $8,024,500$ & $4,464,000 \S$ & 322,700 & 187,000 & 88,400 & $1,465,700$ & 765,600 & $169, ? 00$ & 561,900 \\
\hline 1940 & $6,464,500$ & $3,360,000 €$ & 115,900 & 178,300 & 111,800 & $1,306,300$ & 781,100 & 141,600 & 469,500 \\
\hline 1941 क & $7,442,000$ & $3,840,000 \oint$ & 217,100 & 162,800 & $118,800 \oint$ & $1,670,600$ & 761,200 & 166,600 & 504,900 \\
\hline
\end{tabular}

*Total harvested and unharvested production. See table 4 for averages by five-year periods.

†California production, except Washington included 1927 to date. \# Merchantable and nonmerchantable.

$\oint$ Unofficial estimates of S. W. Shear. $\quad$ ol Preliminary.

Sources of data:

Compiled by S. W. Shear, Giannini Foundation of Agricultural Economics, largely from California and United States Crop Reports, except as noted $\oint$. Cols. 2, 6, and 7: Converted to tons at 48 pounds per bushel except pears other than California at 50 pounds per bushel. Cols. 5 and 9: Dry weight converted to fresh by multiplying figs by 3 , California prunes by $2 \frac{1}{2}$, and other prunes by $3 \frac{1}{2}$. 


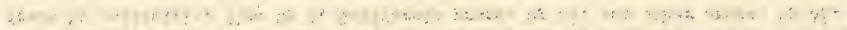

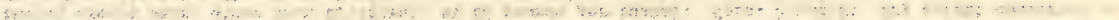

,

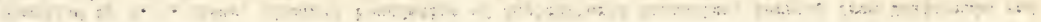

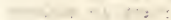

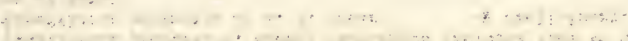

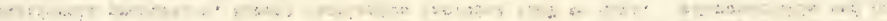

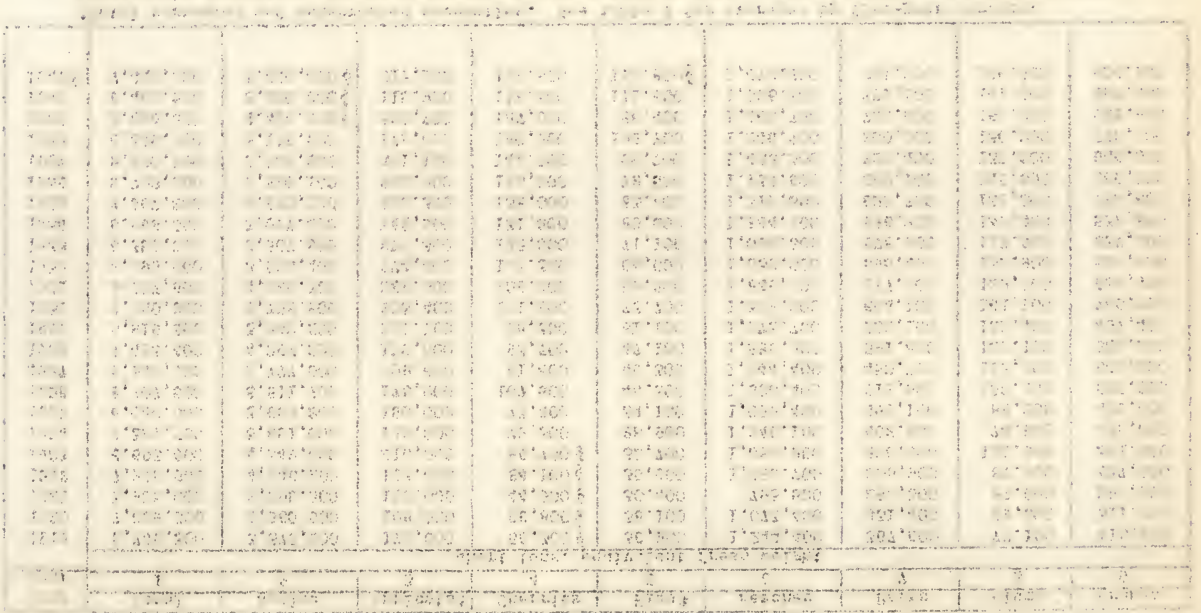

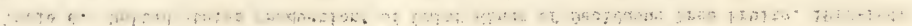


Table 7. California Fruit Production* by Groups -- Citrus, Grapes, and Deciduous Tree, 1919-1941

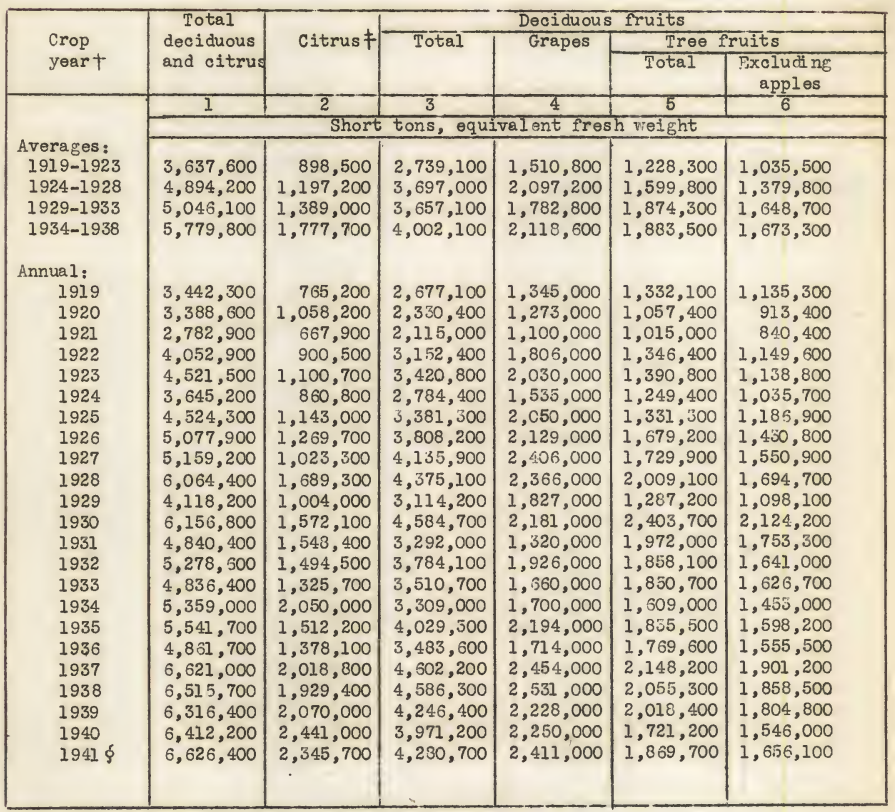

* Includes harvested and unharvested production.

+ Citrus data are for 12 months beginning November 1 of year indicated.

F Includes oranges, grapefruit, and lemons.

$\oint$ Prel iminary.

Sources of data:

Compiled by S. N. Shear, Giannini Foundation of Agricultural

Bconomics, University of California, very largely on the basis of data from United States and California Crop Reports. 


\section{$: \quad, \quad$

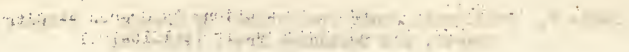

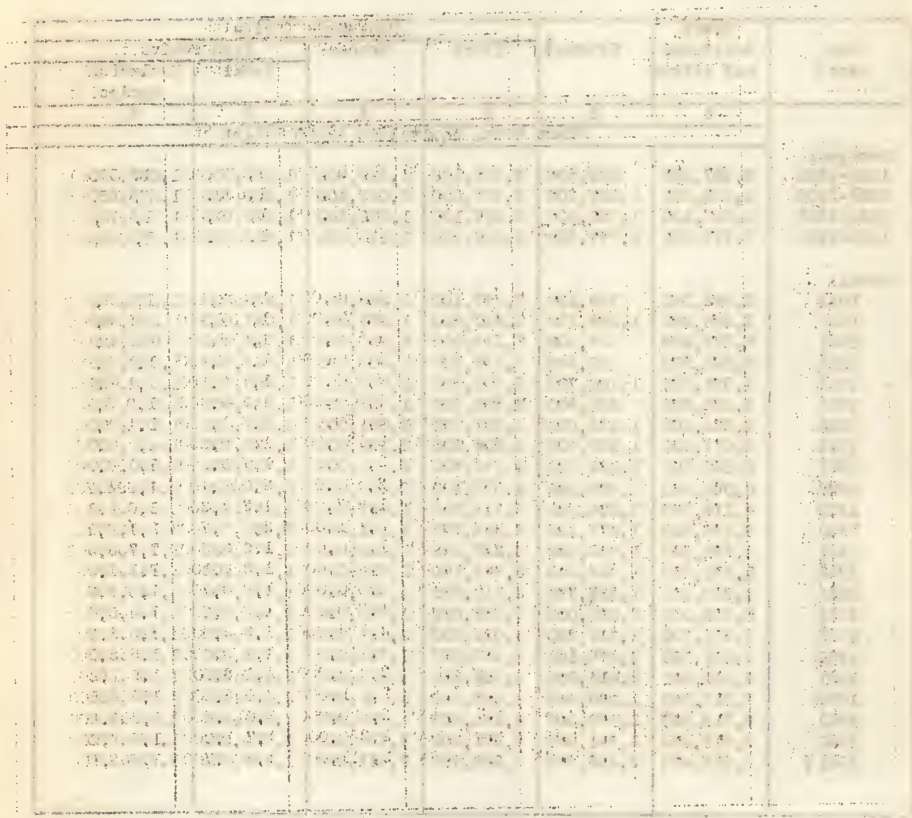

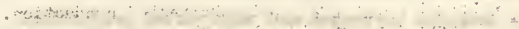

- \&

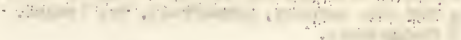

\section{$\because+\cdots+\cdots$}

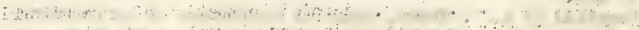

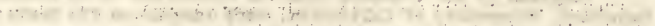

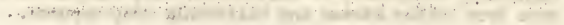


Table 8. California Production* of Chief Kinds of Deciduous Tree Fruits, 1919-1941

\begin{tabular}{|c|c|c|c|c|c|c|c|c|c|}
\hline $\begin{array}{l}\text { Crop } \\
\text { year }\end{array}$ & Total & Apples & Apricots & Cherries & Figst & Peaches & Pears & Plums & Prunes \\
\hline & 1 & 2 & 3 & 4 & 5 & 6 & 7 & 8 & 9 \\
\hline & \multicolumn{9}{|c|}{ Short tons, equivalent fresh weight } \\
\hline 1919 & $1,332,100$ & 196,800 & 172,000 & 12,400 & 36,900 & 411,000 & 111,000 & 42,000 & 350,000 \\
\hline 1920 & $1,057,400$ & 144,000 & 108,000 & 17,500 & 38,100 & 366,000 & 105,000 & 35,000 & 243,800 \\
\hline 1921 & $1,015,000$ & 174,600 & 100,000 & 13,000 & 30,400 & 316,000 & 89,000 & 42,000 & 250,000 \\
\hline 1922 & $1,346,400$ & 196,800 & 163,000 & 17,000 & 35,600 & 421,000 & 150,000 & 48,000 & 315,000 \\
\hline 1923 & $1,390,800$ & 252,000 & 210,000 & 19,000 & 31,800 & 388,000 & 136,000 & 69,000 & 285,000 \\
\hline 1924 & $1,249,400$ & 213,700 & 142,000 & 13,500 & 27,700 & 333,000 & 133,000 & 39,000 & 347,500 \\
\hline 1925 & $1,331,300$ & 144,400 & 150,000 & 12,000 & 31,900 & 396,000 & 181,000 & 51,000 & 365,000 \\
\hline 1926 & $1,679,200$ & 248,400 & 176,000 & 20,000 & 39,300 & 543,000 & 204,000 & 71,000 & 377,500 \\
\hline 1927 & $1,729,900$ & 179,000 & 208,000 & 12,000 & 41,400 & 489,000 & 181,000 & 57,000 & 562,500 \\
\hline 1928 & $2,009,100$ & 314,400 & 175,000 & 16,600 & 40,600 & 618,000 & 226,000 & 66,000 & 552,500 \\
\hline 1929 & $1,287,200$ & 189,100 & 215,000 & 16,300 & 58,300 & 321,000 & 190,000 & 40,000 & 257,500 \\
\hline 1930 & $2,403,700$ & 279,500 & 200,000 & 17,500 & 70,700 & 796,000 & 273,000 & 82,000 & 685,000 \\
\hline 1931 & $1,972,000$ & 218,700 & 277,000 & 23,000 & 57,300 & 579,000 & 217,000 & 65,000 & 535,000 \\
\hline 1932 & $1,858,100$ & 217,100 & 270,000 & 18,500 & 63,500 & 547,000 & 244,000 & 68,000 & 430,000 \\
\hline 1933 & $1,850,700$ & 224,000 & 268,000 & 25,300 & 70,400 & 530,000 & 221,000 & 57,000 & 455,000 \\
\hline 1934 & $1,609,000$ & 156,000 & 139,000 & 17,000 & 79,500 & 495,000 & 233,000 & 62,000 & 427,500 \\
\hline 1935 & $1,835,500$ & 237,300 & 216,000 & 15,000 & 82,200 & 429,000 & 163,000 & 48,000 & 645,000 \\
\hline .1936 & $1,769,600$ & 214,100 & 248,000 & 23,000 & 71,000 & 512,000 & 240,000 & 64,000 & 397,500 \\
\hline 1937 & $2,148,200$ & 247,000 & 311,000 & 21,600 & 98,100 & 558,000 & 224,000 & 66,000 & 622,500 \\
\hline 1938 & $2,055,300$ & $196,800 \xi$ & 166,000 & 30,000 & 105,500 & 492,000 & 282,000 & 63,000 & 720,000 \\
\hline 1939 & $2,018,400$ & $213,600 \dot{0}$ & 312,000 & 36,000 & 87,300 & 583,000 & 253,000 & 71,000 & 462,500 \\
\hline 1940 & $1,721,200$ & $175,200\}$ & 103,000 & 11,000 & 111,000 & 566,000 & 226,000 & 69,000 & 460,000 \\
\hline $1941 \neq$ & $1,869,700$ & $213,600 \hat{s}$ & 205,000 & 20,200 & $117,400 \xi$ & 534,000 & 223,000 & 74,000 & 482,500 \\
\hline
\end{tabular}

† Merchantable and nonmerchantable. $\quad$ Preliminary. $\quad$ Unofficial estimates by S. W. Shear.

Sources of data: Compiled by S. W. Shear, Giannini Foundation of Agricultural Economics, largely from

California and United States Crop Reports except apples, 1938-1941 estimated by S. W. Shear.

Cols. 2, 6, and 7: Apples, pears, peaches converted to tons at 48 pounds per bushel.

Cols. 5 and 9: Dry weight converted to fresh by multiplying figs by 3 and prunes by $2 \frac{1}{2}$. 


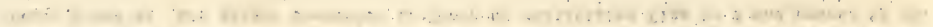

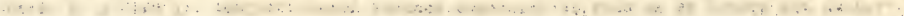

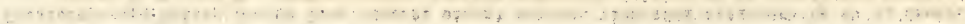

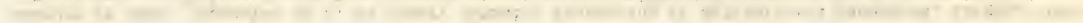

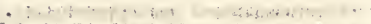

s..

$\because+4 \cdots,+\cdots$

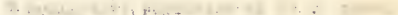

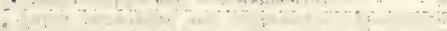

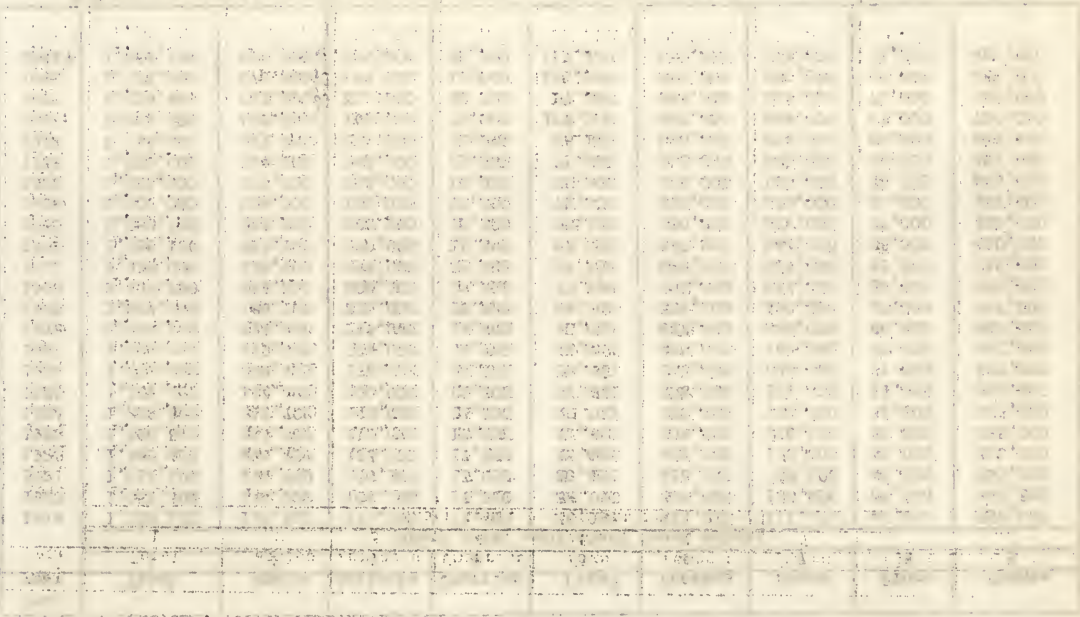

a 
Table 9. United States Bearing Fruit Acreage and Yield Per Bearing Acre by Groups -- Tree and Vine, and Citrus, 1919-1941

\begin{tabular}{|c|c|c|c|c|c|c|c|}
\hline \multirow[b]{2}{*}{ Year } & \multicolumn{3}{|c|}{ Bearing acreage } & \multicolumn{4}{|c|}{ Yield per bearing acre } \\
\hline & $\begin{array}{l}\text { Total } \\
\text { listed }\end{array}$ & Citrus* & $\begin{array}{l}\text { Tree and } \\
\text { vine }+\end{array}$ & $\begin{array}{l}\text { Total } \\
\text { listed }\end{array}$ & Citrus* & $\begin{array}{c}\text { Tree an } \\
\text { vine }\end{array}$ & + \\
\hline & 1 & 2 & 3 & 4 & 5 & 6 & \\
\hline & \multicolumn{3}{|c|}{1,000 acres } & \multicolumn{4}{|c|}{ Tons } \\
\hline $\begin{array}{l}\text { Averages: } \\
1919-1923\end{array}$ & 4,206 & 280 & 3,926 & $2.25 \neq$ & $5.87+$ & 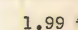 & \\
\hline $1924-1928$ & 4,470 & 408 & 4,062 & $2.49+$ & $5.16+$ & 2.22 & \\
\hline $1929-1933$ & 4,562 & 528 & 4,034 & $2.51+$ & $5.04+$ & 2.18 & \\
\hline $1934-1938$ & 4,504 & 678 & 3,826 & $2.91 \dagger$ & $5.85 \neq$ & $2.38=$ & \\
\hline \multicolumn{8}{|l|}{ Annual: } \\
\hline 1919 & 4,160 & 236 & 3,924 & 2.08 & 5.80 & 1.85 & \\
\hline 1920 & 4,163 & 256 & 3,907 & 2.46 & 6.73 & 2.18 & \\
\hline 1921 & 4,186 & 278 & 3,908 & 1.55 & 4.77 & 1.32 & \\
\hline 1922 & 4,233 & 303 & 3,930 & 2.59 & 5.69 & 2.35 & \\
\hline 1923 & 4,287 & 328 & 3,959 & 2.55 & 6.34 & 2.23 & \\
\hline 1924 & 4,354 & 355 & 3,999 & 2.24 & 4.92 & 2.00 & \\
\hline 1925 & 4,420 & 381 & 4,039 & 2.28 & 5.07 & 2.02 & \\
\hline 1926 & 4,483 & 409 & 4,074 & 2.93 & 5.24 & 2.70 & \\
\hline 1927 & 4,526 & 434 & 4,092 & 2.14 & 4.15 & 1.93 & \\
\hline 1928 & 4,569 & 460 & 4,109 & 2.85 & 6.40 & 2.45 & \\
\hline 1929 & 4,584 & 485 & 4,099 & 2.11 & 3.89 & 1.90 & \\
\hline 1930 & 4,586 & 508 & 4,078 & 2.74 & 6.22 & 2.31 & \\
\hline 1931 & 4,569 & 525 & 4,044 & 2.83 & 5.29 & 2.51 & \\
\hline 1932 & 4,546 & 548 & 3,998 & 2.47 & 5.14 & 2.10 & \\
\hline 1933 & 4,523 & 573 & 3,950 & 2.40 & 4.67 & 2.07 & \\
\hline 1934 & 4,502 & 603 & 3,899 & 2.53 & 6.06 & 1.98 & \\
\hline 1935 & 4,487 & 637 & 3,850 & 2.89 & 4.71 & 2.58 & \\
\hline 1936 & 4,503 & 687 & 3,816 & 2.48 & 5.30 & 1.98 & \\
\hline 1937 & 4,527 & 724 & 3,803 & 3.46 & 6.12 & 2.96 & \\
\hline 1938 & 4,500 & 740 & 3,760 & 3.17 & 7.07 & 2.40 & \\
\hline 1939 & 4,465 & 750 & 3,715 & $3.40 \$$ & 6.36 & 2.80 & $\oint$ \\
\hline 1940 & 4,414 & 752 & 3,662 & $3.29 \$$ & 7.46 & 2.43 & $\oint$ \\
\hline 1941 \$ & 4,352 & 759 & 3,593 & 3.559 & 7.20 & 2.78 & \\
\hline
\end{tabular}

and lemons in California.

† Includes apples, peaches, pears, grapes, plums, prunes, apricots, figs, olives, and avocados. Yields exclude California prunes not harvested.

* Average yields are simple averages of annual data.

$\$$ Partly based on estimates of U. S. total apple: production by S. WT. Shear. कrelininary.

Source of data:

Compiled by S. W. Shear, Giannini Foundation of Agricultural Economics,

from U. S. Dept. Agr., Crops and liarkets, Vol. 18, No. 12, page 298,

December, 1941, except 1939-1940 as noted $\delta$. 


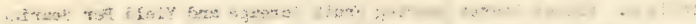
L

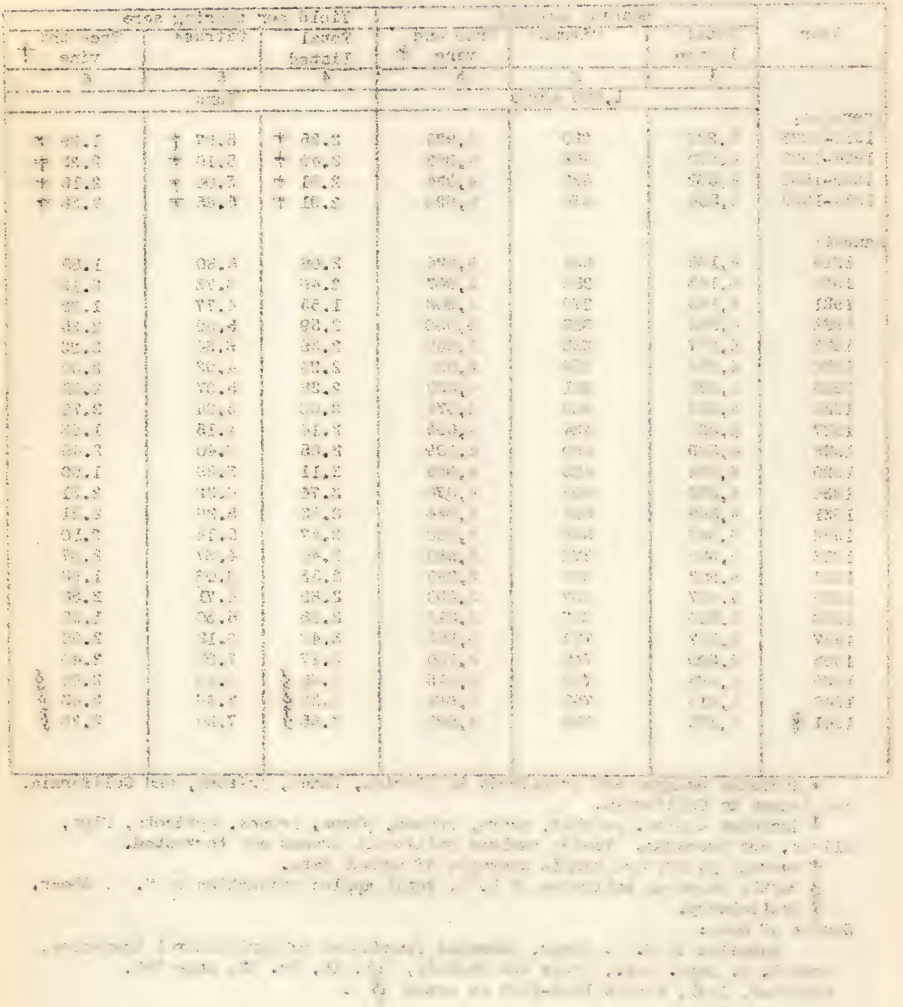


Table 10. California Bearing Acreage* of Fruits by Groups -Citrus, Grapes, and Deciduous Tree, 1919-1941

\begin{tabular}{|c|c|c|c|c|c|c|}
\hline \multirow{3}{*}{$\begin{array}{l}\text { Crop } \\
\text { year }\end{array}$} & \multirow{3}{*}{$\begin{array}{l}\text { Total } \\
\text { deciduous } \\
\text { and citrus }\end{array}$} & \multirow{3}{*}{ Citrust } & \multicolumn{4}{|c|}{ Deciduous fruits } \\
\hline & & & \multirow{2}{*}{ Total } & \multirow{2}{*}{ Grapes } & \multicolumn{2}{|c|}{ Tree fruits } \\
\hline & & & & & Total & $\begin{array}{l}\text { Fixcluding } \\
\text { apples }\end{array}$ \\
\hline \multirow{4}{*}{$\begin{array}{l}\text { Averages: } \\
1919-1923 \\
1924-1928\end{array}$} & 1 & 2 & 3 & 4 & 5 & 6 \\
\hline & \multicolumn{6}{|c|}{ Acres } \\
\hline & & & & & & \\
\hline & $\begin{array}{r}974,512 \\
1,358,012\end{array}$ & $\begin{array}{l}210,655 \\
231,447\end{array}$ & $\begin{array}{r}763,857 \\
1,126,565\end{array}$ & $\begin{array}{l}374,274 \\
604,894\end{array}$ & $\begin{array}{l}389,583 \\
521,671\end{array}$ & $\begin{array}{l}339,948 \\
468,310\end{array}$ \\
\hline $1929-1933$ & $1,367,945$ & 253,812 & $1,114,133$ & 543,411 & 570,722 & 526,750 \\
\hline $1934-1938$ & $1,266,891$ & 280,666 & 986,225 & 485,579 & 500,646 & 461,102 \\
\hline \multicolumn{7}{|l|}{ Annusl: } \\
\hline 1919 & 865,393 & 183,628 & 681,765 & 322,000 & 359,765 & 313,034 \\
\hline 1920 & 921,657 & $205,04.6$ & 715,611 & 346,000 & 369,611 & 322,680 \\
\hline 1921 & 960,630 & 217,836 & 742,794 & 362,000 & 380,794 & 330,896 \\
\hline 1922 & $1,036,804$ & 221,878 & 814,926 & 407,000 & 407,926 & 356,172 \\
\hline 1923 & $1,088,075$ & 223,888 & 864,187 & 434,371 & 429,816 & 376,959 \\
\hline 1924 & $1,194,923$ & 225,398 & 969,525 & 516,698 & 452,827 & 399,055 \\
\hline 1925 & $1,309,345$ & 229,990 & $1,079,355$ & 597,592 & 481,763 & 426,305 \\
\hline 1926 & $1,404,555$ & 232,848 & $1,171,707$ & 643,761 & 524,946 & 468,603 \\
\hline 1927 & $1,429,248$ & 232,455 & $1,196,793$ & 635,464 & 551,329 & 509,987 \\
\hline 1928 & $1,451,987$ & 236,544 & $1,215,443$ & 627,955 & 587,488 & 537,602 \\
\hline 1929 & $1,433,218$ & 243,771 & $1,189,447$ & 606,843 & 582,604 & 535,856 \\
\hline 1930 & $1,375,445$ & 249,468 & $1,125,977$ & 549,862 & 576,115 & 531,638 \\
\hline 1931 & $1,359,699$ & 254,454 & $1,105,245$ & 530,758 & 574,487 & 530,811 \\
\hline 1932 & $1,354,018$ & 250,370 & $1,093,648$ & 525,040 & 568,608 & 525,600 \\
\hline 1933 & $1,317,346$ & 260,999 & $1,056,347$ & 504,552 & 551,795 & 509,845 \\
\hline 1934 & $1,310,422$ & 265,445 & $1,044,977$ & 499,186 & 545,791 & 504,598 \\
\hline 1935 & $1,281,800$ & 271,400 & $1,010,400$ & 491,100 & 519,300 & 476,100 \\
\hline 1936 & $1,220,868$ & 272,680 & 948,188 & 468,468 & 479,720 & 442,174 \\
\hline 1937 & $1,256,584$ & 291,388 & 965,196 & 481,689 & 483,507 & 445,407 \\
\hline 1938 & $1,264,782$ & 302,418 & 962,364 & 487,453 & 474,911 & 437,231 \\
\hline 1939 & $1,264,027$ & 307,798 & 956,229 & 488,428 & 467,801 & 431,565 \\
\hline 1940 & $1,231,474$ & 301,508 & 929,966 & 482,345 & 447,621 & 412,583 \\
\hline $1941 \neq$ & $1,236,300$ & 305,300 & 931,000 & 490,100 & 440,900 & 406,700 \\
\hline
\end{tabular}

* For 1940 county acreage by kinds of fruit and state acreage by variety and age, see R. 5. Blair, and H. C. Phillips, Acreage Estimates of California Fruit and Nut Crops as of 1940, California Cooperative Crop Reporting Service, Sacramento, June, 1941.

† Includes oranges, grapefruit, and lemons.

$\neq$ Preliminary estimates.

Source of data:

Compiled by S. W. Shear, Giannini Foundation of Agricultural Bconomics, University of California, from the latest available estinates of the Califormia Cooperative Crop Reporting Service. 

Table 11. California Bearing Acreage of Chief Kinds of Deciduous Tree Frults, 1919-1941

\begin{tabular}{|c|c|c|c|c|c|c|c|c|c|}
\hline $\begin{array}{l}\text { Crop } \\
\text { year }\end{array}$ & Total & Apples & Apricots & Cherries & Figs & Peaches & Pears & Plums & Prunes \\
\hline & \multicolumn{9}{|c|}{ Acres } \\
\hline 1919 & 359,765 & 46,731 & 46,100 & 8,750 & 10,500 & 103,378 & 23,056 & 17,250 & 104,000 \\
\hline 1920 & 369,611 & 46,931 & 47,907 & 8,884 & 11,023 & 103,200 & 29,366 & 17,300 & 105,000 \\
\hline 1921 & 380,794 & 49,898 & 56,407 & 8,758 & 11,472 & 96,841 & 31,434 & 19,715 & 106,269 \\
\hline 1922 & 407,926 & 51,754 & 60,754 & 9,317 & 12,774 & 101,829 & 37,681 & 22,434 & 111,383 \\
\hline 1923 & 429,816 & 52,857 & 62,287 & 9,646 & 16,979 & 103,856 & 40,962 & 23,800 & 119,429 \\
\hline 1924 & 452,827 & 53,772 & 64,189 & 9,981 & 21,328 & 106,330 & 43,125 & 25,398 & 128,704 \\
\hline 1925 & 481,763 & 55,458 & 66,855 & 10,433 & 23,440 & 111,783 & 46,773 & 28,268 & 138,753 \\
\hline 1926 & 524,946 & 56,343 & 72,107 & 10,828 & 29,537 & 118,079 & 51,993 & 30,081 & 155,978 \\
\hline 1927 & 561,329 & 51,342 & 79,260 & 10,554 & 42,595 & 124,826 & 57,639 & 33,004 & 162,109 \\
\hline 1928 & 587,488 & 49,886 & 82,703 & 11,606 & 47,038 & 134,245 & 60,749 & 33,578 & 167,683 \\
\hline 1929 & 582,604 & 46,748 & 82,136 & 11,984 & 46,353 & 125,832 & 65,637 & 32,584 & 171,330 \\
\hline 1930 & 576,115 & 44,477 & 81,448 & 12,555 & 46,728 & 122,934 & 65,288 & 31,882 & 170,803 \\
\hline 1931 & 574,487 & 43,676 & 80,543 & 13,147 & 46,142 & 122,444 & 66,421 & 31,572 & 170,542 \\
\hline 1932 & 568,608 & 43,008 & 81,534 & 13,621 & 45,760 & 113,628 & 69,580 & 32,119 & 169,358 \\
\hline 1933 & 551,795 & 41,950 & 79,596 & 14,004 & 42,744 & 105,332 & 70,474 & 31,172 & 166,523 \\
\hline 1934 & 545,791 & 41,193 & 78,795 & 14,551 & 41,526 & 103,295 & 68,969 & 31,358 & 166,104 \\
\hline 1935 & 519,300 & 43,200 & 80,000 & 14,200 & 38,000 & 99,000 & 61,000 & 26,900 & 157,000 \\
\hline 1936 & 479,720 & 37,546 & 73,773 & 14,532 & 38,293 & 81,332 & 53,839 & 24,943 & 155,462 \\
\hline 1937 & 483,507 & 38,100 & 74,756 & 15,228 & 38,206 & 81,653 & 53,762 & 25,720 & 156,082 \\
\hline 1938 & 474,911 & 37,680 & 73,571 & 15,211 & 37,689 & 78,132 & 52,801 & 26,032 & 153,795 \\
\hline 1939 & 467,801 & 36,236 & 73,319 & 14,881 & 37,055 & 79,319 & 51,105 & 25,136 & 150,750 \\
\hline 1940 & 447,621 & 35,038 & 70,881 & 13,919 & 35,533 & 77,227 & 46,993 & 21,960 & 146,070 \\
\hline $1941^{*}$ & 440,900 & 34,200 & 69,800 & 13,600 & 35,500 & 77,600 & 45,700 & 22,000 & 142,500 \\
\hline
\end{tabular}

*Preliminary estimates.

Source of data: Compiled by S. W. Shear, Giannini Foundation of Agricultural Economics, University of California, from the latest avallable estimates of the Callfornia Cooperative Crop Reporting Service. 


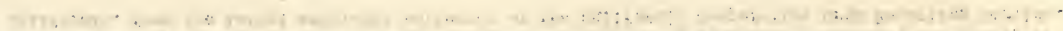

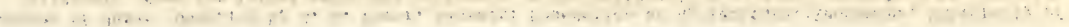

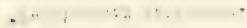

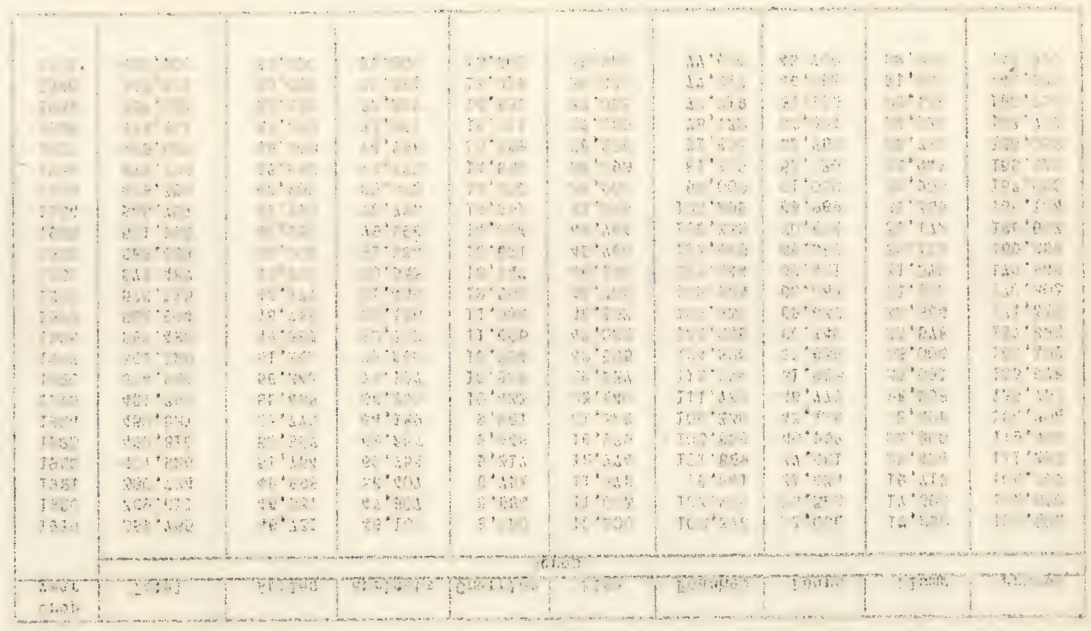

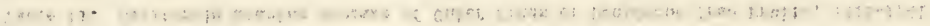


Table 12. California Farm Prices* of Fruits by Groups -Citrus, Grapes, and Deciduous Tree, 1919-1941

\begin{tabular}{|c|c|c|c|c|c|c|}
\hline \multirow{3}{*}{$\begin{array}{l}\text { Crop } \\
\text { yeart }\end{array}$} & \multirow{3}{*}{$\begin{array}{l}\text { Total } \\
\text { deciduous } \\
\text { and citrus }\end{array}$} & \multirow{3}{*}{ Citrus } & \multicolumn{4}{|c|}{ Deciduous fruits } \\
\hline & & & Total & Grapes & Tree & ruits \\
\hline & & & & & Total & $\begin{array}{c}\text { Fxeluding } \\
\text { apples }\end{array}$ \\
\hline \multirow{3}{*}{ Averages: } & 1 & 2 & 3 & 4 & 5 & 6 \\
\hline & \multicolumn{2}{|c|}{ Dollars } & ton, $f r$ & equivale & Weloht & \\
\hline & & & & & & \\
\hline $\begin{array}{l}1919-1923 \\
1924-1928\end{array}$ & 53 & 63 & 49 & 45 & 54 & 59 \\
\hline & 42 & 77 & 31 & 24 & 39 & 41 \\
\hline $1934-1938$ & $\begin{array}{l}32 \\
27\end{array}$ & 51 & 24 & 18 & 29 & 30 \\
\hline & & & & & & \\
\hline $\begin{array}{l}\text { Annual: } \\
1919\end{array}$ & 68 & 81 & 64 & $55 \neq$ & 73 & 79 \\
\hline 1920 & 66 & 57 & 70 & 69 & 71 & 76 \\
\hline 1921 & 60 & 85 & 53 & 58 & 47 & 49 \\
\hline 1922 & 46 & 61 & 42 & 36 & 50 & 56 \\
\hline 1923 & 32 & 46 & 27 & 23 & 33 & 36 \\
\hline 1924 & 49 & 90 & 37 & 32 & 42 & 45 \\
\hline 1925 & 44 & 70 & 35 & 28 & 45 & 47 \\
\hline 1926 & 42 & 76 & 31 & 24 & 39 & 44 \\
\hline 1927 & 46 & 109 & 29 & 24 & 37 & 37 \\
\hline 1928 & 34 & 58 & 24 & 16 & 33 & 36 \\
\hline 1929 & 58 & 115 & 39 & 24 & 62 & 65 \\
\hline 1930 & 29 & 45 & 23 & 16 & 26 & 28 \\
\hline 1931 & 26 & 35 & 22 & 20 & 23 & 24 \\
\hline 1932 & 19 & 32 & 14 & 12 & 16 & 16 \\
\hline 1933 & 29 & 50 & 21 & 16 & 25 & 27 \\
\hline 1934 & 28 & 35 & 23 & 17 & 29 & 30 \\
\hline 1935 & 29 & 54 & 19 & 13 & 27 & 29 \\
\hline 1936 & 35 & 62 & 24 & 19 & 30 & 31 \\
\hline 1937 & 25 & 28 & 24 & 19 & 29 & 31 \\
\hline 1938 & 19 & 27 & 15 & 13 & 19 & 19 \\
\hline 1939 & 24 & 34 & 19 & 14 & 26 & 27 \\
\hline 1940 & 27 & 39 & 20 & 16 & 25 & 26 \\
\hline $1941 \oint$ & 36 & 50 & 29 & 22 & 38 & 39 \\
\hline
\end{tabular}

* Growers ${ }^{\prime}$ returns for naked fruit delivered at growers' first delivery point, except citrus data are net returns to growers for naked fruit delivered at packing-house; averages as well as annual data are weighted vrices. + Citrus data are for 12 months beginning Noverber 1 of year indicated. $\neq$ tixcludes the unknown price of 13,000 tons of raisin grapes marketed fresh. $\$$ Preliminary.

Source of data:

Compiled by S. Whear, Giannini Foundation of Agricultural Jcononies, University of Califomia, by dividing data in tahle 14 by harvested projuction as reported largely in reports of the Jnited States and Jalifornia Crop Reporting Services. 


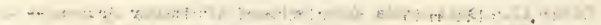

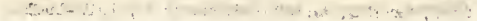

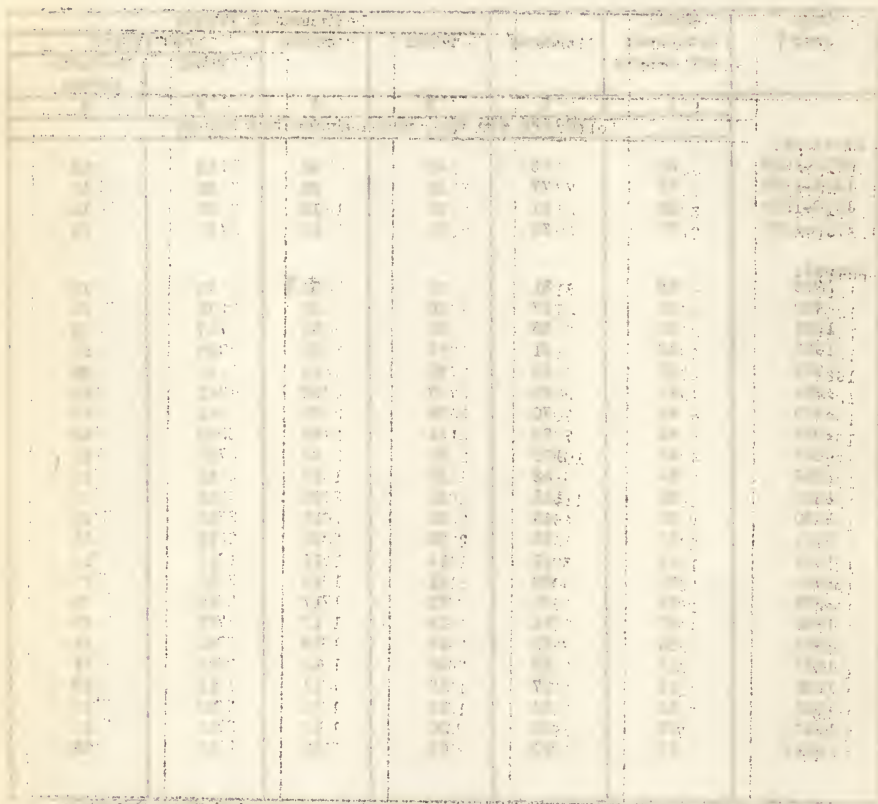

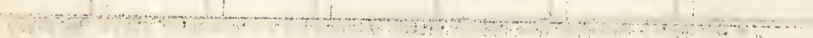

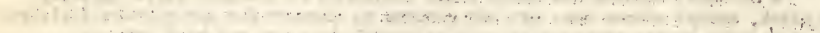

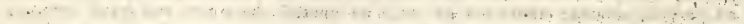

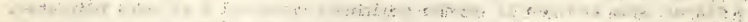

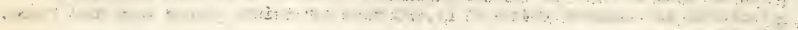

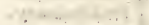

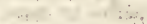

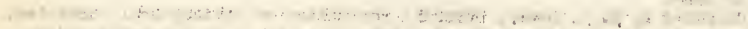

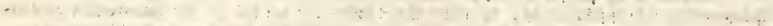

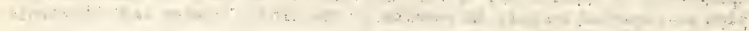

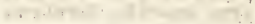


Table 13. California Farm Prices* of Chief Kinds of Deciduous Tree Fruits, 1919-1941

\begin{tabular}{|c|c|c|c|c|c|c|c|c|c|}
\hline $\begin{array}{l}\text { Crop } \\
\text { year }\end{array}$ & Total & Apples & Apricots & Cherries & Figst & Peaches & Pears & Plums & Prunes \\
\hline \multirow[b]{2}{*}{ - } & 1 & 2 & 3 & 4 & 5 & 6 & 7 & 8 & 9 \\
\hline & \multicolumn{9}{|c|}{ Dollars per ton, fresh equivalent weight } \\
\hline 1919 & 73 & 38 & 87 & 150 & $52 \neq$ & 65 & 72 & 74 & 96 \\
\hline 1920 & 71 & 44 & 87 & 200 & $32 \neq$ & 69 & 90 & 102 & 68 \\
\hline 1921 & 47 & 38 & 56 & 125 & $53 \neq$ & 31 & 62 & 58 & 58 \\
\hline 1922 & 50 & 16 & 76 & 180 & $44 \neq$ & 44 & 50 & 45 & 60 \\
\hline 1923 & 33 & 18 & 31 & 160 & $37 \neq$ & 25 & 50 & 34 & 40 \\
\hline 1924 & 42 & 25 & 52 & 140 & $39^{\circ}$ & 31 & 66 & 57 & 44 \\
\hline 1925 & 45 & 33 & 61 & 160 & 43 & 34 & 52 & 47 & 48 \\
\hline 1926 & 39 & 16 & 68 & 180 & 44 & 38 & 35 & 29 & 40 \\
\hline 1927 & 37 & 33 & 57 & 180 & 26 & 25 & 54 & 53 & 30 \\
\hline 1928 & 33 & 19 & 51 & 150 & 26 & 22 & 37 & 45 & 40 \\
\hline 1929 & 62 & 44 & 64 & 190 & 39 & 58 & 69 & 91 & 64 \\
\hline 1930 & 26 & 16 & 39 & 148 & 24 & 20 & 23 & 35 & 25 \\
\hline 1931 & 23 & 18 & 29 & 93 & 19 & 17 & 24 & 26 & 20 \\
\hline 1932 & 16 & 10 & 18 & 60 & 11 & 10 & 12 & 18 & 22 \\
\hline 1933 & 25 & 17 & 30 & 66 & 18 & 19 & 21 & 26 & 32 \\
\hline 1934 & 29 & 19 & 54 & 90 & 23 & 25 & 32 & 32 & 24 \\
\hline 1935 & 27 & 15 & 46 & 125 & 20 & 27 & 28 & 39 & 23 \\
\hline 1936 & 30 & 17 & 38 & 102 & 30 & 27 & 27 & 30 & 32 \\
\hline 1937 & 29 & 13 & 37 & 170 & 28 & 34 & 28 & 49 & 22 \\
\hline 1938 & 19 & 14 & 35 & 84 & 25 & 13 & 14 & 28 & 17 \\
\hline 1939 & 26 & 14 & 33 & 79 & 28 & 21 & 26 & 31 & 27 \\
\hline 1940 & 25 & 14 & 53 & 141 & 28 & 20 & 26 & 41 & 22 \\
\hline $1941 \hat{\oint}$ & 38 & 23 & 45 & 127 & $41 \neq$ & 42 & 40 & 50 & 28 \\
\hline
\end{tabular}

* Growers' returns for naked fruit at growers' first delivery point.

tPrice of all figs, including merchantable and nonmerchantable.

F Partly based on unofficial estimates by S. W. Shear. $\oint$ Preliminary.

Sources of data: Compiled by S. W. Shear, Giannini Foundation of Agricultural Economics, University of California, very largely on the basis of reports of the United States and California Crop Reporting Services. Col. 1 is weighted price obtained by dividing data in table 15, col. 1, by harvested production. 
$2 ; \cdots, \cdots$

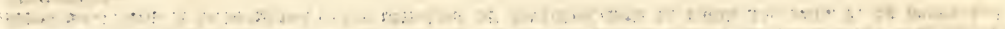

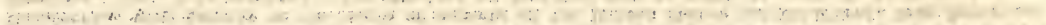

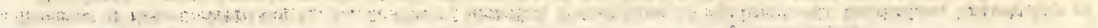

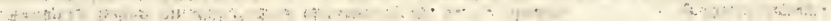

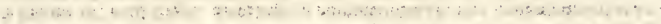

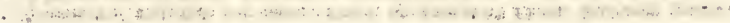

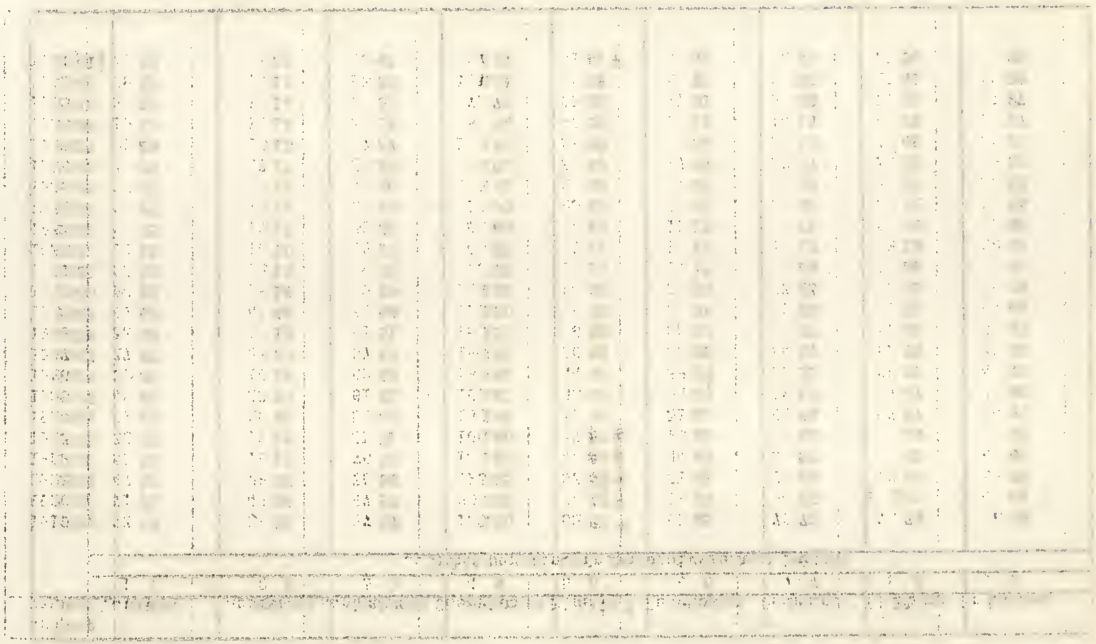

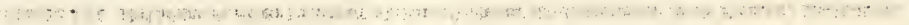


Table 14. California Total Farm Value* of Fruits by Groups -Citrus, Grapes, and Deciduous Tree, 1919-1941

\begin{tabular}{|c|c|c|c|c|c|c|}
\hline \multirow{3}{*}{$\begin{array}{l}\text { Cro } p \\
\text { year }+\end{array}$} & \multirow{3}{*}{$\begin{array}{l}\text { Total } \\
\text { deciduous } \\
\text { and citrus }\end{array}$} & \multirow{3}{*}{ Citrus } & \multicolumn{4}{|c|}{ Deciduous fruits } \\
\hline & & & \multirow[t]{2}{*}{ Total } & \multirow[t]{2}{*}{ Grapes } & \multicolumn{2}{|c|}{ Tree fruits } \\
\hline & & & & & Total & $\begin{array}{c}\text { Fxcluding } \\
\text { apples }\end{array}$ \\
\hline \multirow[b]{3}{*}{ Averages: } & 1 & 2 & 3 & 4 & 5 & 6 \\
\hline & \multicolumn{6}{|c|}{ Thousands of dollars } \\
\hline & & & & & & \\
\hline $\begin{array}{l}1919-1923 \\
1924-1928\end{array}$ & 190,135 & 56,921 & 133,214 & 66,539 & 66,675 & 61,066 \\
\hline & 202,614 & 92,768 & 109,846 & 48,987 & 60,859 & 55,644 \\
\hline $\begin{array}{l}1929-1933 \\
1934-1938\end{array}$ & $\begin{array}{l}149,216 \\
150,388\end{array}$ & 70,480 & 78,736 & 30,207 & 48,529 & 43,976 \\
\hline & & 67,726 & 82,662 & 33,951 & 48,711 & 45,533 \\
\hline \multicolumn{7}{|l|}{ Annual: } \\
\hline 1919 & 232,523 & 61,617 & 170,906 & $73,430 \neq$ & 97,476 & 90,096 \\
\hline 1920 & 223,790 & 60,792 & 162,998 & 87,555 & 75,443 & 69,143 \\
\hline 1921 & 167,868 & 56,608 & 111,260 & 63,540 & 47,720 & 41,172 \\
\hline 1922 & 183,360 & 54,706 & 128,654 & 61,470 & 67,184 & 63,986 \\
\hline 1923 & 143,135 & 50,882 & 92,253 & 46,700 & 45,553 & 40,933 \\
\hline 1924 & 179,397 & 77,668 & 101,729 & $49,3 \in 0$ & 52,369 & 47,027 \\
\hline 1925 & 194,822 & 80,545 & 114,277 & 54,040 & 60,237 & 55,424 \\
\hline 1926 & 213,170 & 96,405 & 116,765 & 50,465 & 66,300 & 62,367 \\
\hline 1927 & 228,596 & 111,642 & 116,954 & 55,530 & 61,424 & 55,458 \\
\hline 1928 & 197,083 & 97,577 & 99,506 & 35,538 & 63,968 & 57,942 \\
\hline 1929 & 238,089 & 115,352 & 122,737 & 43,390 & 79,347 & 71,073 \\
\hline 1930 & 158,724 & 70,008 & 88,716 & 33,649 & 55,067 & 50,526 \\
\hline 1931 & 121,600 & 54,934 & 66,666 & 26,503 & 40,163 & 36,154 \\
\hline 1932 & 92,683 & 47,309 & 45,374 & 20,755 & 24,609 & 22,403 \\
\hline 1933 & 134,981 & 64,796 & 70,185 & 26,727 & 43,458 & 39,725 \\
\hline 1934 & 144,348 & 70,574 & 73,774 & 29,520 & 44,254 & 41,329 \\
\hline 1935 & 157,987 & 79,780 & 78,207 & 28,344 & 49,863 & 46,402 \\
\hline 1936 & 168,359 & 83,112 & 85,247 & 32,741 & 52,506 & 48,848 \\
\hline 1937 & 164,804 & 55,662 & 109,142 & 46,690 & 62,452 & 59,351 \\
\hline 1938 & 116,440 & 49,502 & 66,938 & 32,459 & 34,479 & 31,733 \\
\hline 1939 & 148,772 & 67,915 & 80,857 & 30,523 & 50,334 & 47,562 \\
\hline 1940 & 170,024 & 93,698 & 76,326 & 35,125 & 41,201 & 39,023 \\
\hline 1941 \& & 238,201 & 116,157 & 122,044 & 53,411 & 68,633 & 63,958 \\
\hline & & & & & & \\
\hline
\end{tabular}

* Growers returns for naked fruit delivered at frowers first del ivery point, except citrus data are net returns to growers for naked fruit delivered at packing-house.

tCitrus data are for 12 months beginning November 1 of year indicated. $\$$ Excludes the unknown value of 13,000 tons of rais in grapes marketer fresh. $\oint$ Preliminary.

Sources of data:

Compiled by S. W. Shear, Giannini Foundation of Agricultural Fconomics.

Col. 2: From U.S.D.A., Agr. Ikt. Serv., Production, Disposition, and

Value of Citrus Fruits, Crop Seasons, 1909-10 to 1938-39, hiay, 1941, except

1936 to date based on reports of U.S. and California Crop Reporting Services. Col. 4: From Grapes, table 5, col. 1.

Cols. 5 and 6: Table 15, cols. 1 and 2 . 


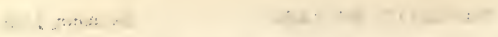

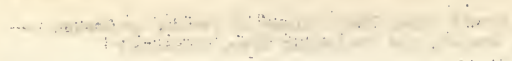

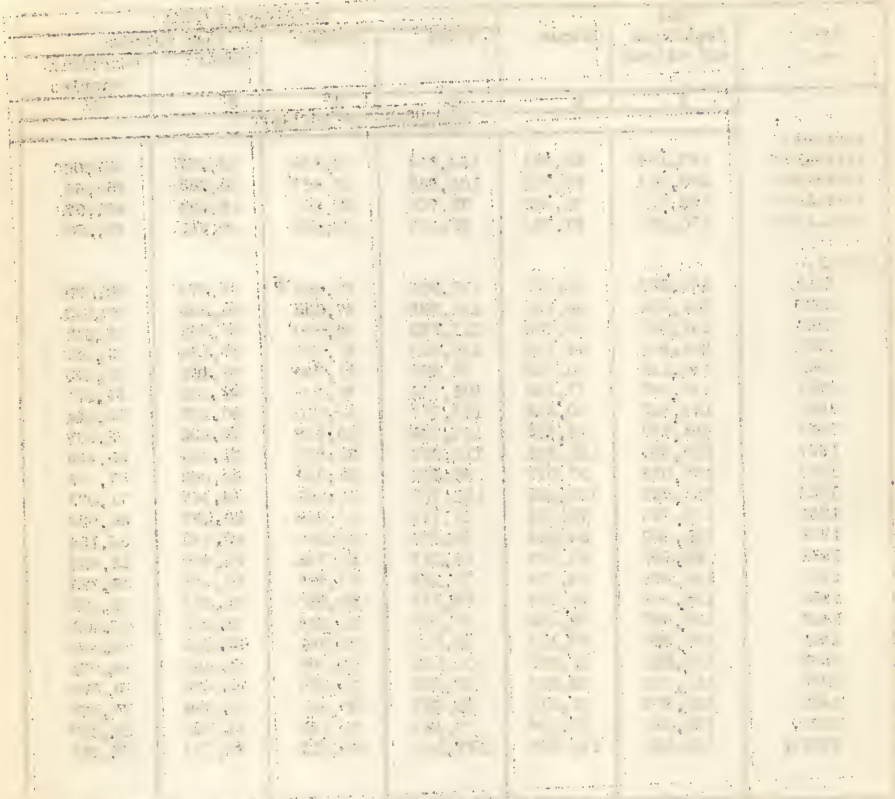

-

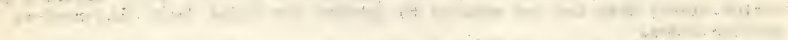

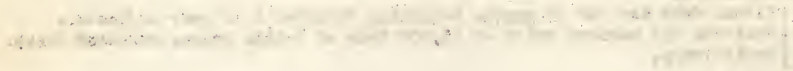

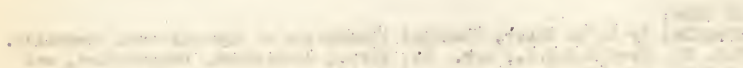

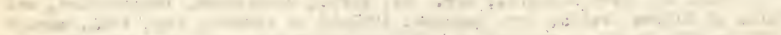

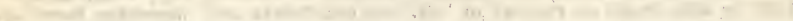


Table 15. California Total Farm Value* of Chief Kinds of Deciduous Tree Fruits, 1919-1941

\begin{tabular}{|c|c|c|c|c|c|c|c|c|c|}
\hline $\begin{array}{l}\text { Crop } \\
\text { year }\end{array}$ & Total & Apples & Apricots & Cherries & Figst & Peaches & Pears & Plums & Prunes \\
\hline & 1 & 2 & 3 & 4 & 5 & 6 & 7 & 8 & 9 \\
\hline & \multicolumn{9}{|c|}{ Thousands of dollars } \\
\hline 1919 & 97,476 & 7,380 & 14,964 & 1,860 & $1,920 \neq$ & 26,652 & 7,992 & 3,108 & 33,600 \\
\hline 1920 & 75,443 & 6,300 & 9,396 & 3,500 & $1,227 \neq$ & 25,340 & 9,450 & 3,570 & 16,660 \\
\hline 1921 & 47,720 & 6,548 & 5,600 & 1,625 & $1,605 \neq$ & 9,888 & 5,518 & 2,436 & 14,500 \\
\hline 1922 & 67,184 & 3,198 & 12,388 & 3,060 & $1,580 \neq$ & 18,398 & 7,500 & 2,160 & 18,900 \\
\hline 1923 & 45,553 & 4,620 & 6,510 & 3,040 & $1,185 \neq$ & 9,652 & 6,800 & 2,346 & 11,400 \\
\hline 1924 & 52,369 & 5,342 & 7,384 & 1,890 & 1,079 & 10,383 & 8,778 & 2,223 & 15,290 \\
\hline 1925 & 60,237 & 4,813 & 9,150 & 1,920 & 1,366 & 13,659 & 9,412 & 2,397 & 17,520 \\
\hline 1926 & 66,300 & 3,933 & 11,968 & 3,600 & 1,711 & 20,789 & 7,140 & 2,059 & 15,100 \\
\hline 1927 & 61,424 & 5,966 & 11,856 & 2,160 & 1,080 & 10,800 & 9,666 & 3,021 & 16,875 \\
\hline 1928 & 63,968 & 6,026 & 8,925 & 2,490 & 1,049 & 12,120 & 8,288 & 2,970 & 22,100 \\
\hline 1929 & 79,347 & 8,274 & 13,760 & 3,097 & 2,260 & 18,726 & 13,110 & 3,640 & 15,480 \\
\hline 1930 & 55,067 & 4,541 & 7,476 & 2,590 & 1,701 & 13,880 & 5,566 & 2,870 & 16,443 \\
\hline 1931 & 40,163 & 4,009 & 7,917 & 1,860 & 1,095 & 8,226 & 4,848 & 1,508 & 10,700 \\
\hline 1932 & 24,609 & 2,206 & 4,626 & 960 & 721 & 3,652 & 2,160 & 1,044 & 9,240 \\
\hline 1933 & 43,458 & 3,733 & 8,040 & 1,643 & 1,240 & 9,141 & 3,801 & 1,300 & 14,560 \\
\hline 1934 & 44,254 & 2,925 & 7,506 & 1,530 & 1,861 & 11,020 & 7,168 & 1,984 & 10,260 \\
\hline 1935 & 49,863 & 3,461 & 9,936 & 1,875 & 1,608 & 11,583 & 4,564 & 1,872 & 14,964 \\
\hline 1936 & 52,506 & 3,658 & 9,424 & 2,346 & 2,134 & 13,824 & 6,480 & 1,920 & 12,720 \\
\hline 1937 & 62,452 & 3,101 & 11,507 & 3,672 & 2,796 & 18,760 & 5,936 & 3,234 & 13,446 \\
\hline 1938 & 34,479 & $2,746 \neq$ & 5,810 & 2,117 & 2,673 & 6,321 & 3,640 & 1,764 & 9,408 \\
\hline 1939 & 50,334 & $2,772 \div$ & 10,032 & 2,607 & 2,485 & 11,611 & 6,448 & 1,984 & 12,395 \\
\hline 1940 & 41,201 & $2,178 \neq$ & 5,459 & 1,551 & 3,102 & 11,020 & 5,642 & 2,624 & 9,625 \\
\hline 19413 & 68,633 & $4,675 \ddagger$ & 9,225 & 2,565 & 4,8337 & 22,407 & 8,920 & 3,450 & 12,558 \\
\hline
\end{tabular}

* Growers' returns for naked fruit delivered at growers' first delivery point.

+ Includes merchantable and nonmerchantable figs.

F Partly basen on unofficial estimates by S. W. Shear. $\quad \dot{\rho}$ Preliminary.

Sources of data: Compiled by S. W. Shear, Giannini Foundation of Agricuitural Economics, University of California, largely calculated from data on harvested production and unit farm price as given

in reports of the United States and California Crop Reporting Services. (See tables on each fruit.) 
$\therefore \quad \div \quad \ldots+\cdots$

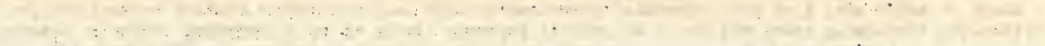

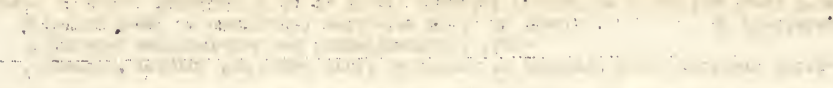

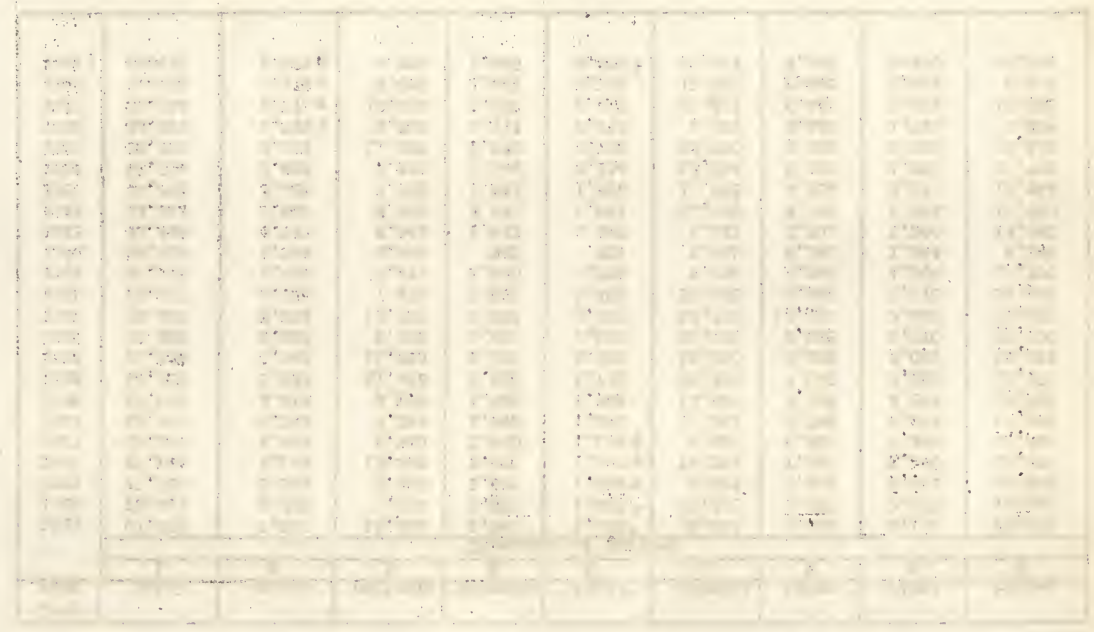

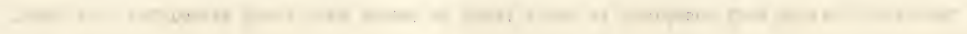


Table 16. Pacific Coast Dried Fruit Production, Five-Year Averages 1894-1938 and Annuel 1932-1941

\begin{tabular}{|c|c|c|c|c|c|c|c|c|c|c|}
\hline \multirow[b]{2}{*}{$\begin{array}{l}\text { Crop } \\
\text { year }\end{array}$} & \multirow[b]{2}{*}{$\begin{array}{l}\text { Pacific Coast } \\
\text { totel* }\end{array}$} & \multirow[b]{2}{*}{$\begin{array}{c}\text { Nor thwest } \\
\text { total* }\end{array}$} & \multicolumn{8}{|c|}{ California } \\
\hline & & & Total & Apples & Apricots & $\mathrm{Figs}^{+}$ & Peaches & Fears & Prunes & $\begin{array}{l}\text { Raisins and other } \\
\text { dried grapes } \neq\end{array}$ \\
\hline Averages: & \multicolumn{10}{|c|}{ Short tons, unprocessed dry weight } \\
\hline $1894-1898$ & & & 105,500 & 2,200 & 8,400 & 1,500 & 10,900 & 3,500 & 35,300 & 43,700 \\
\hline $1899-1903$ & 167,900 & 8,000 & 159,900 & 3,200 & 11,400 & 3,100 & 18,500 & 3,700 & 73,200 & 46,800 \\
\hline $1904-1908$ & 146,500 & 7,200 & 139,300 & 2,600 & 6,700 & 3,300 & 15,500 & 1,600 & 54,600 & 55,000 \\
\hline $1909-1913$ & 201,000 & 10,900 & 190,100 & 3,000 & 14,100 & 4,700 & 23,100 & 1,400 & 72,100 & 71,700 \\
\hline $1914-1918$ & 291,200 & 15,600 & 275,600 & 5,600 & 16,300 & 8,500 & 31,700 & 1,100 & 76,200 & 136,200 \\
\hline $1919-1923$ & 413,600 & 25,300 & 388,300 & 9,000 & 16,500 & 10,900 & 27,000 & 3,100 & 115,500 & 206,300 \\
\hline 1924-1928 & 513,700 & 27,100 & 486,600 & 9,800 & 20,000 & 10,600 & 22,900 & 4,000 & 176,400 & 242,900 \\
\hline $1929-1933$ & 524,100 & 38,800 & 485,300 & 10,700 & 31,200 & 19,100 & 21,700 & 5,100 & 185,600 & 211,900 \\
\hline $1934-1938$ & 555,600 & 31,600 & 524,000 & 10,500 & 26,200 & 25,500 & 23,400 & 5,800 & 212,200 & 220,400 \\
\hline \multicolumn{11}{|l|}{ Annual: } \\
\hline 1932 & 559,800 & 34,500 & 525,300 & 9,800 & 35,300 & 19,000 & 22,200 & 5,500 & 168,000 & 265,500 \\
\hline 1933 & 514,900 & 32,000 & 482,900 & 13,300 & 37,500 & 21,500 & 23,400 & 7,000 & 182,000 & $19^{\circ}, 200$ \\
\hline 1934 & 463,200 & 38,600 & 424,600 & 8,800 & 16,800 & 23,500 & 25,800 & 4,900 & 171,000 & 173,800 \\
\hline 1935 & 599,200 & 49,300 & 549,900 & 12,500 & 25,800 & 24,000 & 19,500 & 6,100 & 258,000 & 204,000 \\
\hline 1936 & 471,100 & 30,300 & 440,800 & 11,700 & 32,200 & 20,000 & 26,400 & 8,100 & 159,000 & 183,400 \\
\hline 1937 & 614,800 & 15,700 & 599,100 & 12,000 & 34,400 & 28,700 & 23,000 & 3,500 & 249,000 & 248,500 \\
\hline 1938 & 629,700 & 24,300 & 605,400 & 7,500 & 21,500 & 31,500 & 22,200 & 6,500 & 224,000 & 292,200 \\
\hline 1939 & 581,500 & 38,400 & 543,100 & 10,900 & 41,000 & 26,000 & 24,900 & 8,100 & 185,000 & 247,200 \\
\hline 1940 & 431,000 & 8,200 & 422,800 & 4,800 & 11,300 & 32,000 & 24,400 & 3,100 & 175,000 & 172,200 \\
\hline 1941$\}$ & 478,000 & 12,400 & 465,600 & 8,500 & 19,700 & 33,000 & 14,800 & 3,600 & 176,000 & 210,000 \\
\hline
\end{tabular}

* Northwest data include only prunes, 1899 through 1918; apples and prunes since 1918. Apples, the only fruit dried commercially in significant quantities in states other than Pacific Cosst, averaged 27,000 tons, 1909-1913; $10,240,1919-1923 ; 10,200,1924-1928 ; 7,110,1929-1933$; and 3,300, 1934-1938. See Apples, table 6.

+Includes merchantable and nonmerchantable figs.

fDried grapes other than raisins are included from 1926 to date. S Preliminary estimates.

Sources of data: Compiled by S. W. Shear, Giannini Foundation of Agriculturai Economics, University of California, lergely from reports of Dried Fruit Association of California and of Cilifornia Cooperative Crop Reporting Service for recent years, and tra de estimates from Californie Fruit News and other sources before 1919. California data for 1941 are from report of Dried Fruit Assn., March 13, 1942, and differ somewhat from most of the preliminary 
$\therefore \quad \therefore \quad \therefore \quad \cdots+\cdots$

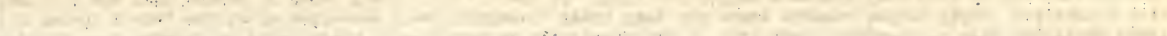
.

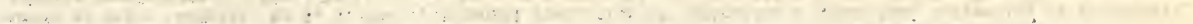

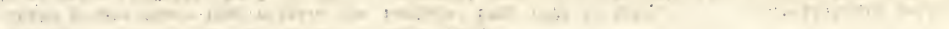

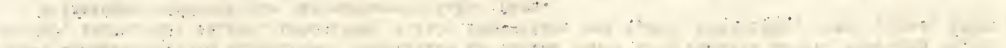

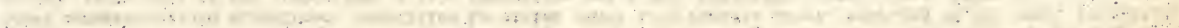

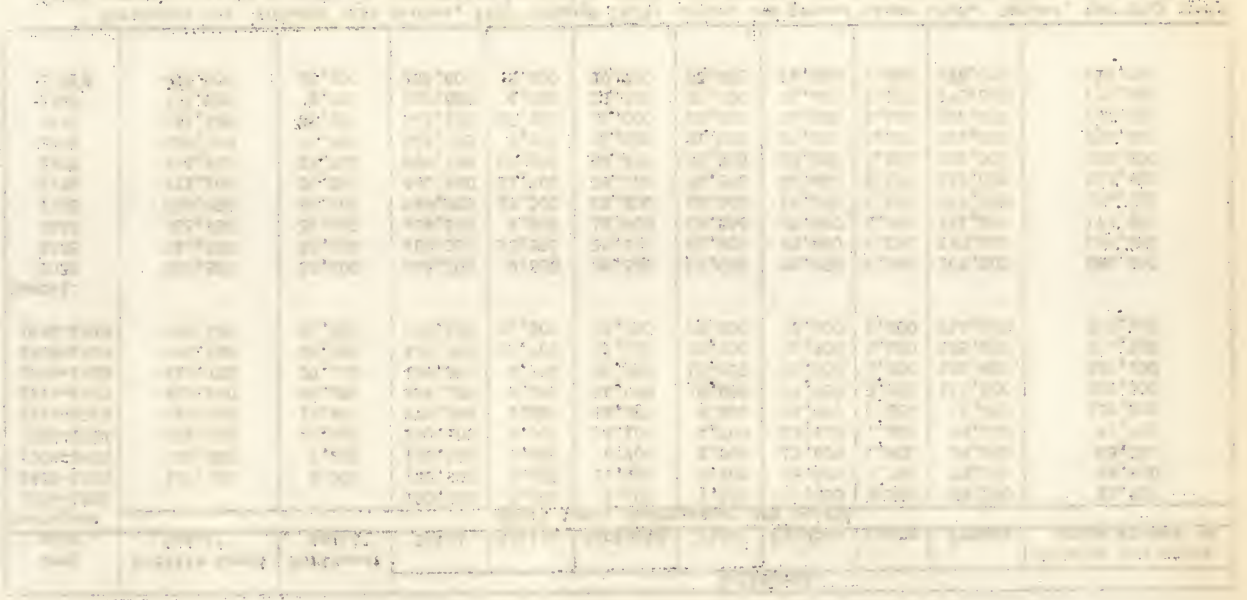


January 1942

CONPARATIVE SUI ARIES

Table 17

F.0.B. California Packers' Frices of Dried Fruits, 1921-1941

\begin{tabular}{|c|c|c|c|c|c|c|c|}
\hline Year & Applas & Apricots & Figs & Peaches & Pears & Prunes & Raisins \\
\hline & 1 & 2 & 3 & 4 & 5 & 6 & 7 \\
\hline & \multicolumn{7}{|c|}{ Cents per pound } \\
\hline Averages : & & & & & & & \\
\hline $1921-1925$ & 10.8 & 17.9 & 7.1 & 10.9 & 13.8 & 8.5 & 9.3 \\
\hline $1920-1929$ & 11.2 & 17.9 & 6.7 & 11.2 & 11.2 & 7.1 & 5.4 \\
\hline $1930-1933$ & 7.4 & 9.4 & 5.2 & 6.5 & 6.6 & 4.2 & 4.3 \\
\hline $1934-1938$ & $7 \cdot 9$ & 14.0 & 6.8 & 8.1 & 7.0 & 4.1 & 4.2 \\
\hline \multicolumn{8}{|l|}{ A nnual: } \\
\hline 1921 & 13.0 & 20.4 & 8.8 & 11.4 & 15.4 & 9.0 & 14.0 \\
\hline 1922 & 9.8 & 23.6 & 9.1 & 11.8 & 13.1 & 10.0 & 10.5 \\
\hline 1923 & 8.0 & 9.7 & 5.8 & 7.5 & 8.2 & 8.0 & 7.3 \\
\hline 1924 & 12.0 & 16.3 & 6.1 & 10.0 & 17.3 & 7.5 & 7.4 \\
\hline 1925 & 11.0 & 19.6 & 5.7 & $13 \cdot 8$ & 14.9 & 7.8 & 7.3 \\
\hline 1926 & 8.5 & 22.1 & 6.2 & 12.5 & 9.1 & ن. 7 & 6.8 \\
\hline 1927 & $12 \cdot 5$ & 16.2 & 5.0 & 9.4 & 10.4 & 5.6 & 5.6 \\
\hline 1928 & 11.5 & 16.4 & 6.2 & 8.4 & 10.0 & 6.6 & 4.4 \\
\hline 1929 & 12.5 & 16.9 & 9.4 & 14.5 & 15.1 & $9 \cdot 6$ & 4.9 \\
\hline 1930 & 8.5 & 11.4 & 5.9 & 6.8 & 7.1 & 4.4 & 4.7 \\
\hline 1931 & $7 \cdot 5$ & 8.4 & 5.5 & 6.8 & 6.6 & 3.4 & 5.1 \\
\hline 1932 & 5.3 & 7.3 & 3.7 & 4.7 & 5.7 & 3.5 & 3.3 \\
\hline 1933 & 8.3 & 10.4 & 5.7 & 7.7 & 6.8 & 5.7 & 4.2 \\
\hline 1934 & 9.0 & 17.9 & 7.0 & 8.7 & 7.6 & 5.1 & 4.3 \\
\hline 1935 & 7.5 & $14.0 ิ$ & 6.3 & 9.0 & 7.7 & 3.5 & 4.1 \\
\hline 1936 & 9.5 & 13.0 & 8.0 & 8.7 & 7.3 & 5.0 & 4.8 \\
\hline 1937 & 6.5 & 10.6 & 6.6 & 7.3 & 5.9 & 3.6 & 4.0 \\
\hline 1938 & 7.0 & 13.9 & 0.1 & 3.9 & 6.4 & 3.3 & 3.7 \\
\hline 1939 & 6.8 & 11.0 & 7.7 & 8.1 & 7.3 & 4.4 & 3.4 \\
\hline 1940 & 5.4 & 14.4 & 7.7 & 6.5 & 6.0 & 4.0 & 4.3 \\
\hline $1941 *$ & 12.8 & 15.3 & 12.6 & 13.4 & 8.1 & $5.8 *$ & $5.7 *$ \\
\hline
\end{tabular}

* Preliminary estimates.

Sources of data: Compiled by S. W. Shear, Giannini Foundation of Agricultural Economics, from weekly issues of the California Fruit News.

Col. 1: Choice dried apples, August-December inclúsive.

Col. 2: Choice Blenheims, July-December inclusive.

Col. 3: Choice Adriatics, season through December 31.

Col. 4: Choice Juirs, August-December inclusive.

Col. 5: Choice Northern Bartletts, seas on through December 31

Col. 6: Monthly prices of 50/60's French for crop years weighted by shipments.

Col. 7: Thompson seedless, choice bulk, monthly prices for crop years weighted by shipments. 

Table 18. United States Dried Fruit Production, Exports, Imports, and Consumption* Average Crop Yesirs, 1934-1938t

\begin{tabular}{|c|c|c|c|c|c|c|c|c|c|c|c|c|c|c|c|}
\hline \multirow[b]{3}{*}{$\begin{array}{l}\text { Kind of } \\
\text { fruit }\end{array}$} & \multirow{2}{*}{\multicolumn{3}{|c|}{$\begin{array}{l}\text { California } \\
\text { Production }\end{array}$}} & \multicolumn{12}{|c|}{ United States, continental and noncontiguous territory } \\
\hline & & & & Pro & ducti & on & Cor & nsumpti & ion* & Dome & stic e & xportst & For & ign imp & rts \\
\hline & $\begin{array}{l}\text { Short } \\
\text { tons } \neq\end{array}$ & $\begin{array}{l}\text { Per } \\
\text { cent } \\
\text { of } \\
\text { total } \\
\end{array}$ & $\begin{array}{l}\text { Per } \\
\text { cent } \\
\text { of } \\
\text { U.S. }\end{array}$ & $\begin{array}{c}\text { Short } \\
\text { tons }\end{array}$ & $\begin{array}{l}\text { Per } \\
\text { cent } \\
\text { of } \\
\text { total }\end{array}$ & $\begin{array}{l}\text { Per } \\
\text { capite } \\
\text { pounds }\end{array}$ & $\begin{array}{l}\text { Short } \\
\text { tons } \neq\end{array}$ & \begin{tabular}{|l|} 
Per \\
cent \\
of \\
total
\end{tabular} & $\begin{array}{l}\text { Per } \\
\text { capita, } \\
\text { pounds }\end{array}$ & $\begin{array}{l}\text { Short } \\
\text { tons } \neq\end{array}$ & \begin{tabular}{|l|} 
Per \\
cent \\
of \\
totel
\end{tabular} & $\begin{array}{l}\text { Per } \\
\text { cent } \\
\text { of U.S. } \\
\text { pro- } \\
\text { duction }\end{array}$ & $\begin{array}{l}\text { Short } \\
\text { tons } \neq\end{array}$ & $\begin{array}{l}\text { Per } \\
\text { cent } \\
\text { of } \\
\text { total }\end{array}$ & $\begin{array}{l}\text { Per } \\
\text { cent } \\
\text { of con- } \\
\text { sump- } \\
\text { tion }\end{array}$ \\
\hline Total & 525,700 & 100.0 & 93.8 & 560,700 & 100.0 & 8.70 & 371,300 & 100.0 & 5.76 & 201,800 & 100.0 & 36.0 & 32,700 & 100.0 & 8.8 \\
\hline Raisins & 218,600 & 41.6 & 100.0 & 218,600 & 39.0 & 3.39 & 145,500 & 39.2 & 2.26 & 65,800 & 32.6 & 30.1 & 400 & 1.2 & 0.3 \\
\hline Currants & 9 & 9 & 4 & 9 & 9 & 9 & 3,000 & 0.8 & 0.04 & -11 & --1 & -1 & 3,000 & 9.2 & 100.0 \\
\hline Prunes & 212,200 & 40.4 & 90.2 & 235,300 & 42.0 & 3.65 & 122,400 & 33.0 & 1.90 & 97,300 & 48.2 & $41.4^{\prime}$ & $\ldots * *$ & $-\cdots * *$ & $-\ldots * *$ \\
\hline Peaches & 23,400 & 4.4 & 100.0 & 23,400 & 4.2 & 0.36 & 18,700 & 5.0 & 0.29 & 4,400 & 2.2 & 18.8 & $--* *$ & $--* *$ & $--* *$ \\
\hline Apples & 10,500 & 2.0 & 47.1 & 22,300 & 4.0 & 0.35 & 8,500 & 2.3 & 0.13 & 13,800 & 6.8 & 61.9 & $-\cdots * *$ & $-n^{* *}$ & $-{ }^{* * *}$ \\
\hline Apricots & 26,200 & 5.0 & 100.0 & 26,200 & 4.7 & 0.40 & 13,400 & 3.6 & 0.21 & 12,800 & 6.4 & 48.9 & $--* *$ & $-\cdots * *$ & $\ldots *$ \\
\hline Figs & 25,500 & 4.8 & 100.0 & 25,500 & 4.5 & 0.40 & 28,300 & 7.6 & 0.44 & -1 & - & -1 & 2,800 & 8.6 & 9.9 \\
\hline Pears & 5,800 & 1.1 & 100.0 & 5,800 & 1.0 & 0.09 & 1,400 & 0.4 & 0.02 & 4,400 & 2. & $75.9^{\prime \prime}$ & $-\cdots^{* *}$ & $-z^{* *}$ & $-\ldots * *$ \\
\hline Dates & 3,500 & 0.7 & 97.2 & 3,600 & 0.6 & 0.06 & 30,100 & 8.1 & 0.47 & -11 & 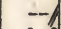 & - & 26,500 & 81.0 & 88.0 \\
\hline Other // & - & - & - & - & - & - & & & 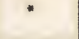 & $\begin{array}{l}3,300 \\
\neq 0\end{array}$ & & & ** & $\ldots * *$ & $-n^{* * *}$ \\
\hline
\end{tabular}

* Consumption is production minus exports plus imports, except prunes, raisins, and peaches are U. S. shipments to domestic markets including relief but not diversion, plus imports for raisins. Consumption of individual fruits includes consumption in dried fruit salad, and "total" consumption includes 3,300 tons of "other" fruit exported.

+ Crop year data except apple and "other" exports, and fig and date imports are for years beginning July 1.

f Unprocessed weight except apple exports, "other" exports, and date imports. (Data rounded to hundred tons.)

California production of merchantable and nonmerchantable figs.

9 Production of California currants is included with raisins and averaged about 1,100 tons.

/" "Other" applies to exports only and consists of dried fruits not specified separately in customs reports, including figs, dates, nectarines, dried apple waste (except pomace), and cherries.

** Almost no dried fruits other than raisins, currants, figs, and dates are imported.

\# Exports of individual fruits include exports of the fruit as dried fruit salad.

Sources of data: Compiled by S. W. Shear, Giannini Foundation of Agricultural Economics largely from reports of U. S. Dept. Agr., Dept. Commerce, and Dried Fruit Association of California. 


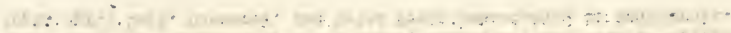

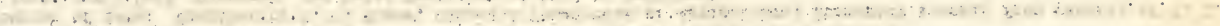

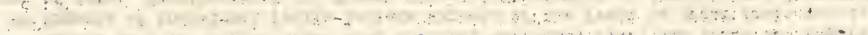

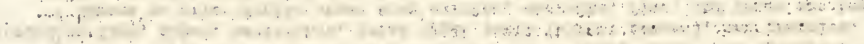

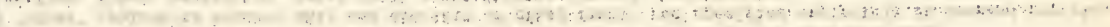

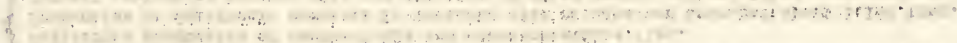

?

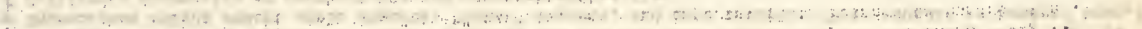

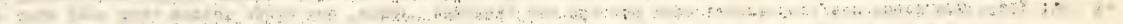

i

4.

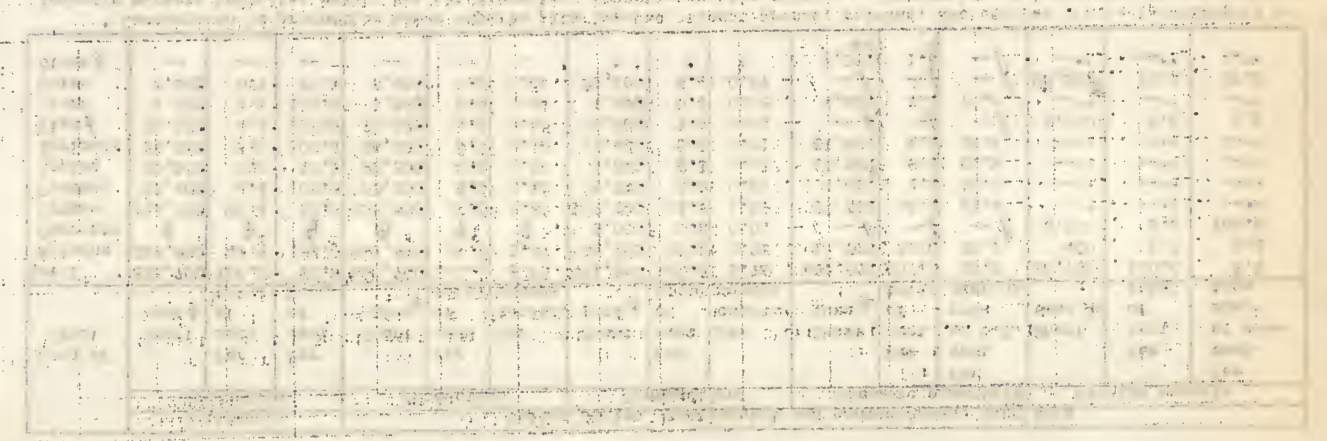


Table 19. United States Domestic Exports of Dried Fruits by Kinds, 1924-1940

\begin{tabular}{|c|c|c|c|c|c|c|c|c|c|c|c|}
\hline \multirow{3}{*}{$\begin{array}{l}\text { Year } \\
\text { begin- } \\
\text { ning } \\
\text { July } 1\end{array}$} & \multirow{3}{*}{$\begin{array}{l}\text { Grand } \\
\text { total }\end{array}$} & \multirow[b]{3}{*}{ Raisins } & \multicolumn{9}{|c|}{ Deciduous tree fruits } \\
\hline & & & \multirow[b]{2}{*}{ Total } & \multirow[b]{2}{*}{ Apples* } & \multirow[b]{2}{*}{ Peaches } & \multirow[b]{2}{*}{ Prunes } & \multirow[b]{2}{*}{ Apricots } & \multicolumn{4}{|c|}{ Other } \\
\hline & & & & & & & & Total & rears & $\begin{array}{l}\text { Fruit } \\
\text { salad }\end{array}$ & $\begin{array}{c}\text { All } \\
\text { other* }\end{array}$ \\
\hline \multirow{2}{*}{\multicolumn{12}{|c|}{ Averages: }} \\
\hline & & & & & & & & & & & \\
\hline $1924-1928$ & 218,942 & 79,300 & 139,642 & 14,845 & 3,397 & 103,240 & 9.766 & 8,394 & --+ & - & -- \\
\hline $1929-1933$ & 212,575 & 58,247 & 154,328 & 16,735 & 3,604 & 106,837 & 15,125 & 12,027 & 3,244 & $\ldots$ & - \\
\hline $1934-1938$ & 200,953 & 60,985 & 139,968 & 13,341 & 3,408 & 96,313 & 13,268 & 13,638 & 3,489 & 6,870 & 3,279 \\
\hline \multicolumn{12}{|l|}{ Annual: } \\
\hline 1924 & 155,491 & 45,392 & 110,099 & 9,612 & 2,334 & 85,886 & 6,646 & 5,621 & - & - & - \\
\hline 1925 & 172,378 & 67,514 & 104,864 & 12,416 & 1,676 & 75,702 & 9,066 & 6,004 & - & - & - \\
\hline 1926 & 201,440 & 76,168 & 125,272 & 16,335 & 3,484 & 87,772 & 8,951 & 8,730 & - & - & - \\
\hline 1927 & 262,757 & 96,550 & 166,207 & 10,852 & 3,271 & 130,312 & 11,842 & 9,930 & - & -- & - \\
\hline 1928 & 302,646 & 110,878 & 191,768 & 25,012 & 6,218 & 136,526 & 12,326 & 11,686 & 1,31 丮 & - & - \\
\hline 1929 & 167,025 & 64,348 & 102,677 & 11,884 & 1,924 & 71,495 & 9,550 & 7,824 & 1,828 & $666 \oint$ & 5,330 \\
\hline 1930 & 258,170 & 62,550 & 195,620 & 19,060 & 4,241 & 148,127 & 11,824 & 12,368 & 4,019 & 7,259 & 1,090 \\
\hline 1931 & 234,196 & 61,106 & 173,090 & 15,778 & 4,245 & 121,968 & 18,811 & 12,288 & 3,040 & 7,780 & 1,468 \\
\hline 1932 & 199,258 & 56,254 & 143,004 & 18,301 & 3,824 & 91,177 & 17,134 & 12,568 & 3,128 & 7,054 & 2,386 \\
\hline 1933 & 204,226 & 46,977 & 157,249 & 18,652 & 3,785 & 101,416 & 18,308 & 15,088 & 4,204 & 5,978 & 4,906 \\
\hline 1934 & 156,528 & 46,945 & 109,583 & 11,748 & 3,176 & 76,358 & 7,598 & 10,703 & 2,843 & 5,698 & 2,162 \\
\hline 1935 & 210,484 & 54,508 & 155,976 & 16,285 & 3,048 & 108,820 & 13,294 & 14,529 & 3,780 & 7,236 & 3,513 \\
\hline 1936 & 178,548 & 56,166 & 122,382 & 10,639 & 3,522 & 81,976 & 14,683 & 11,562 & 3,676 & 4,494 & 3,392 \\
\hline 1937 & 223,024 & 70,685 & 152,339 & 12,218 & 3,175 & 107,396 & 16,342 & 13,208 & 2,789 & 6,489 & 3,930 \\
\hline 1938 & 236,180 & 76,622 & 159,558 & 15,815 & 4,119 & 107,016 & 14,420 & 18,188 & 4,358 & 10,431 & 3,399 \\
\hline 1939 9 & 162,058 & 63,385 & 98,673 & 8,370 & 2,580 & 59,523 & 15,960 & 12,240 & 2,466 & 7,806 & 1,968 \\
\hline 1940 & 70,472 & 43,166 & 27,306 & 1,334 & 802 & 19,017 & 1,454 & 4,699 & 393 & 1,791 & 2,515 \\
\hline
\end{tabular}

"Since January 1, 1933, apple weste (except pomace) included in "all other" and excluded from "apples." +Dashes indicute data not available. \& Includes exports for January-Jure, 1930, only. fincludes exports for January-June, 1929, only. \$ Preliminary.

Source of data: Compiled by S. W. Shear, Giannini Foundation, from official data of U. S. Bur. For. and Dom. Com., as compiled largely by Office of Foreign Agricultural Relations, U. S. Dept. Agriculture. 


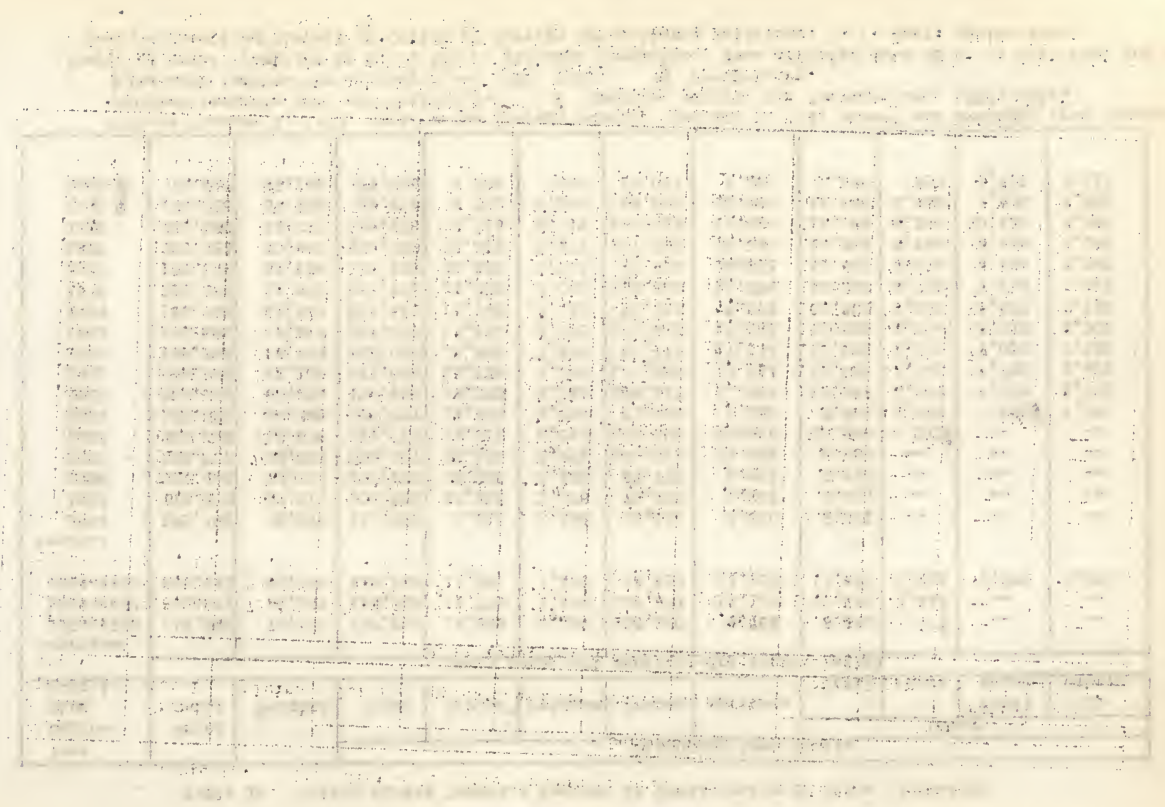

$\therefore{ }^{+}+$ 
Table 20. United States Domestic Exports of Canned Fruits by Kinds, 1924-1940

\begin{tabular}{|c|c|c|c|c|c|c|c|c|c|}
\hline $\begin{array}{l}\text { Year } \\
\text { beginning } \\
\text { July } 1\end{array}$ & Total & $\begin{array}{c}\text { Apples } \\
\text { including } \\
\text { sauce }\end{array}$ & Apricots & Grapefruit & Peaches & Pears & Pineapples & $\begin{array}{l}\text { Fruits } \\
\text { for salad }\end{array}$ & Other \\
\hline & \multirow{2}{*}{\multicolumn{9}{|c|}{ Short tons, net declared canned weight }} \\
\hline Averages: & & & & & & & & & \\
\hline $1924-1928$ & 132,397 & 8,396 & 15,206 & $-\infty$ & 41,052 & 33,115 & 19,998 & - & 14,630 \\
\hline $1929-1933$ & 134,097 & 8,059 & 11,924 & - & 37,299 & 33,978 & 14,029 & 16,126 & 12,682 \\
\hline $1934-1938$ & 145,396 & 7,404 & 12,104 & 17,267 & 36,666 & 35,699 & 11,403 & 18,564 & 6,289 \\
\hline \multicolumn{10}{|l|}{ Annual: } \\
\hline 1924 & 100,616 & 6,744 & 15,680 & - & 28,695 & 26,925 & 13,126 & - & 9,446 \\
\hline 1925 & 133,336 & 6,456 & 14,773 & - & 41,580 & 37,938 & 18,771 & - & 13,818 \\
\hline 1926 & 135,185 & 8,100 & 17,948 & - & 40,948 & 33,052 & 18,713 & - & 16,424 \\
\hline 1927 & 127,938 & 6,873 & 14,506 & - & 43,317 & 26,336 & 25,614 & - & 11,292 \\
\hline 1928 & 164,912 & 13,808 & 13,124 & - & 50,719 & 41,326 & 23,767 & $8,276+$ & 13,892 \\
\hline 1929 & 141,858 & 10,030 & 16,617 & - & 37,235 & 27,354 & 23,154 & 15,074 & 12,394 \\
\hline 1930 & 135,548 & 7,684 & 9,512 & $3,152 \neq$ & 37,881 & 37,177 & 17,654 & 15,810 & 6,678 \\
\hline 1931 & 124,912 & 8,337 & 11,581 & 3,324 & 33,150 & 35,785 & 10,460 & 16,101 & 6,174 \\
\hline 1932 & 119,292 & 8,970 & 9,752 & 7,400 & 37,500 & 30,381 & 7,962 & 13,467 & $3,860^{\circ}$ \\
\hline 1933 & 148,876 & 5,272 & 12,157 & 15,949 & 40,732 & 39,192 & 10,915 & 20,179 & 4,480 \\
\hline 1934 & 118,250 & 6,734 & 5,274 & 14,433 & 25,209 & 35,689 & 9,556 & 15,577 & 5,778 \\
\hline 1935 & 170,406 & 10,794 & 13,377 & 14,961 & 51,479 & 41,158 & 11,846 & 20,213 & 6,578 \\
\hline 1936 & 135,574 & 6,032 & 11,866 & 17,268 & 30,122 & 33,441 & 11,939 & 19,154 & 5,752 \\
\hline 1937 & 128,140 & 5,962 & 13,465 & 17,378 & 28,194 & 29,563 & 12,592 & 16,301 & 4,685 \\
\hline 1938 & 174,608 & 7,500 & 16,538 & 22,294 & 48,326 & 38,644 & 11,082 & 21,574 & 8,650 \\
\hline 1939 \& & 167,541 & 6,463 & 16,572 & 26,851 & 43,205 & 30,959 & 10,251 & 23,194 & 10,046 \\
\hline $1940 \$$ & 8,291 & 352 & 305 & 240 & 1,982 & 1,224 & 792 & 1,656 & 1,740 \\
\hline
\end{tabular}

* Dashes indicate data not reported separately, but included in "other."

+ Includes exports for January-June, 1929, only.

F Includes exports for January-June, 1931, only.

S Preliminary.

Source of data: Compiled by S. W. Shear, Giannini Foundation of Agricultural Economics, University of California, from official data of U. S. Bur. For. and Dom. Com., as compiled largely by office of Foreign Agricultural Relations, U. S. Dept. Agrí culture. 


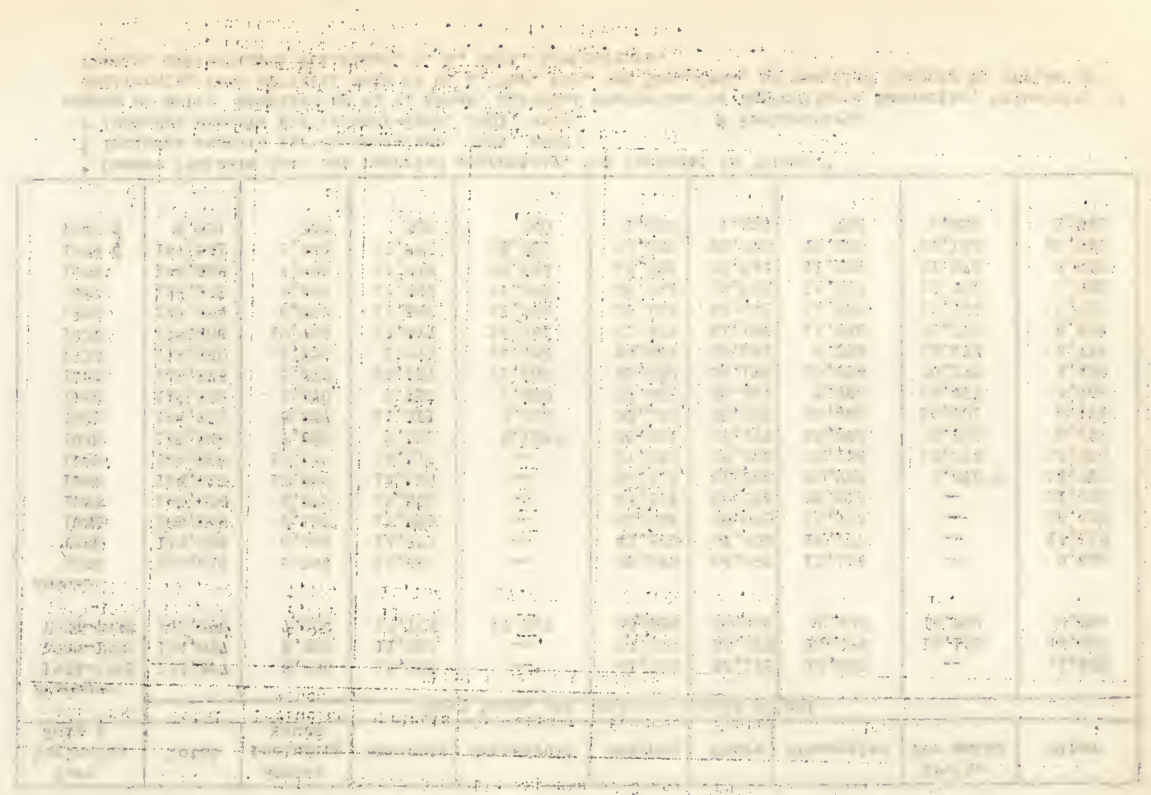

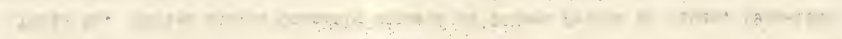

Int 
COMP ARATIVE SUMMARIES

January, 1942

Table 21. Canned Facks of California Chief Fruits and Hawiian Pineapple, Averages 1909-1938, Annual 1924-1941

\begin{tabular}{|c|c|c|c|c|c|c|c|c|c|c|}
\hline \multirow{2}{*}{$\begin{array}{l}\text { Crop } \\
\text { gear* }\end{array}$} & \multicolumn{9}{|c|}{ California } & \multirow{2}{*}{$\begin{array}{c}\text { Hawail, } \\
\text { pineapples }\end{array}$} \\
\hline & Total & Peaches & Apricots & Pears & Figs & Cherries & Plums & Grapes & $\begin{array}{l}\text { Cocktail } \\
\text { and salad }\end{array}$ & \\
\hline \multirow{2}{*}{$\begin{array}{l}\text { Averages: } \\
1909-1913\end{array}$} & \multicolumn{10}{|c|}{ Thousands of cases, equivalent 24 no. $2 \frac{1}{2}$ cans $\neq$} \\
\hline & 4,3756 & 2,366 & 916 & 670 & -5 & 240 & 133 & 50 & $\infty$ & 915 \\
\hline $1914-1918$ & 7,0736 & 4,146 & 1,588 & 859 & - & 253 & 146 & 81 & - & 2,800 \\
\hline $1919-1923$ & 11,5450 & 7,180 & 2,495 & 1,140 & $-\infty$ & 474 & 172 & 84 & -- & 5,397 \\
\hline $1924-1928$ & $16,193 \oint$ & 11,150 & 2,448 & 1,929 & 134 & 271 & 152 & 109 & - & 8,407 \\
\hline $1929-1933$ & 15,885 & 9,312 & 2,441 & 1,809 & 157 & 306 & 114 & 63 & 1,683 & 9,548 \\
\hline $1934-1938$ & 19,663 & 10,719 & 2,987 & 1,918 我 & 278 & 205 & 109 & 108 & 3,339 & 10,743 \\
\hline \multicolumn{11}{|l|}{ Annual: } \\
\hline 1924 & $9,923 \phi$ & 6,141 & 1,968 & 1,400 & 51 & 200 & 85 & 78 & - & 6,826 \\
\hline 1925 & $15,019\}$ & 10,143 & 2,094 & 2,200 & 78 & 210 & 168 & 126 & - & 8,729 \\
\hline 1926 & $21,359^{\circ}$ & 14,059 & 3,227 & 1,957 & 163 & 510 & 214 & 116 & 1,113 & 8,940 \\
\hline 1927 & 17,228 & 10,813 & 2,960 & 1,861 & 167 & 165 & 132 & 136 & 994 & 8,879 \\
\hline 1928 & 20,911 & 14,596 & 1,991 & 2,226 & 210 & 268 & 163 & 90 & 1,367 & 8,663 \\
\hline 1929 & 16,698 & 8,100 & 4,023 & 2,021 & 215 & 382 & 116 & 128 & 1,713 & 9,210 \\
\hline 1930 & 19,639 & 13,294 & 1,954 & 1,871 & 228 & 369 & 183 & 80 & 1,660 & 12,672 \\
\hline 1931 & 13,967 & 8,421 & 2,006 & 1,809 & 74 & 200 & 91 & 17 & 1,349 & 12,808 \\
\hline 1932 & 11,494 & 6,438 & 1,805 & 1,418 & 142 & 184 & 69 & 33 & 1,405 & 5,064 \\
\hline 1933 & 17,628 & 10,309 & 2,416 & 1,928 & 124 & 393 & 110 & 58 & 2,290 & 7,985 \\
\hline 1934 & 16,227 & 8,598 & 1,774 & 2,663 & 216 & 160 & 109 & 131 & 2,576 & 9,690 \\
\hline 1935 & 19,358 & 11,216 & 3,164 & 1,387 & 211 & 133 & 124 & 101 & 3,022 & 10,380 \\
\hline 1936 & 20,404 & $10,711 "$ & 2,899 & 2,415 & 310 & 201 & 77 & 103 & 3,688 & 10,923 \\
\hline 1937 & 25,716 & $13,248^{\circ}$ & 5,553 & 1,499 & 406 & 240 & 180 & 114 & 4,476 & 12,203 \\
\hline 1938 & 16,612 & $9,822^{4}$ & 1,547 & 1,626 & 246 & 294 & 56 & 89 & 2,932 & 10,521 \\
\hline 1939 & 22,300 & 11,4624 & 3,338 & 1,347 & 220 & 469 & 85 & 121 & 5,258 & 10,992 \\
\hline 1940 & 19,750 & 10,742 & 1,815 & 1,532 & 434 & 87 & 54 & 124 & 4,962 & 11,056 \\
\hline 1941 & 25,449 & $12,733^{\circ}$ & 4,072 & 1,792 & 703 & 147 & 102 & 159 & 5,741 & $*$ \\
\hline
\end{tabular}

- Data are for crop years except cocktail and salad pack are for calendar years. + Salad and cocktail packed partly from canned pack of the individual fruits as given. Equivalent cases except cherry, peach, pear, and plum, $1909-1918$, and probably pineapple data are actual cases. Dashes indicate data not available and to tals exclude data for figs and cocktail and salad in certain years. Ancludes small quantities of Northwest pears packed in California 1935-1941. cludes pickled cases: 1936, 115,619; 1937, 111,280; 1938, 24,944; 1939, 195,681; 1940, 156,$665 ; 1941,187,014 . *$ * The canned pineapple pack for the year beginning June 1, 1941 will be slightly smaller than for 1940 . Sources of data:

Compiled by S. W. Shear, Giannini Foundation, based on California Canners League reports, California Crop Report, 1927, es and U.S. Census of Manufactures, except pineapples 1909-1932 from reports of Association of Hawailan Pineapple Packers, and 1933-1940 Pineapple Producers Cooperative Assn. pack. 


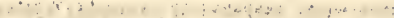

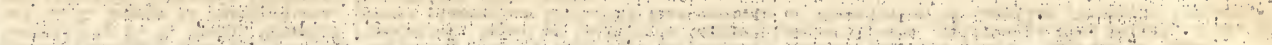

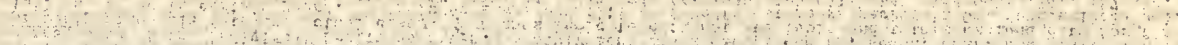

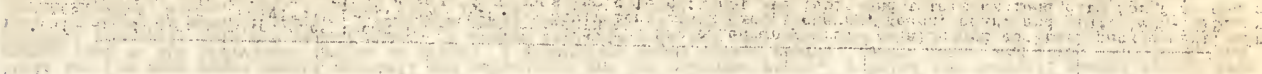

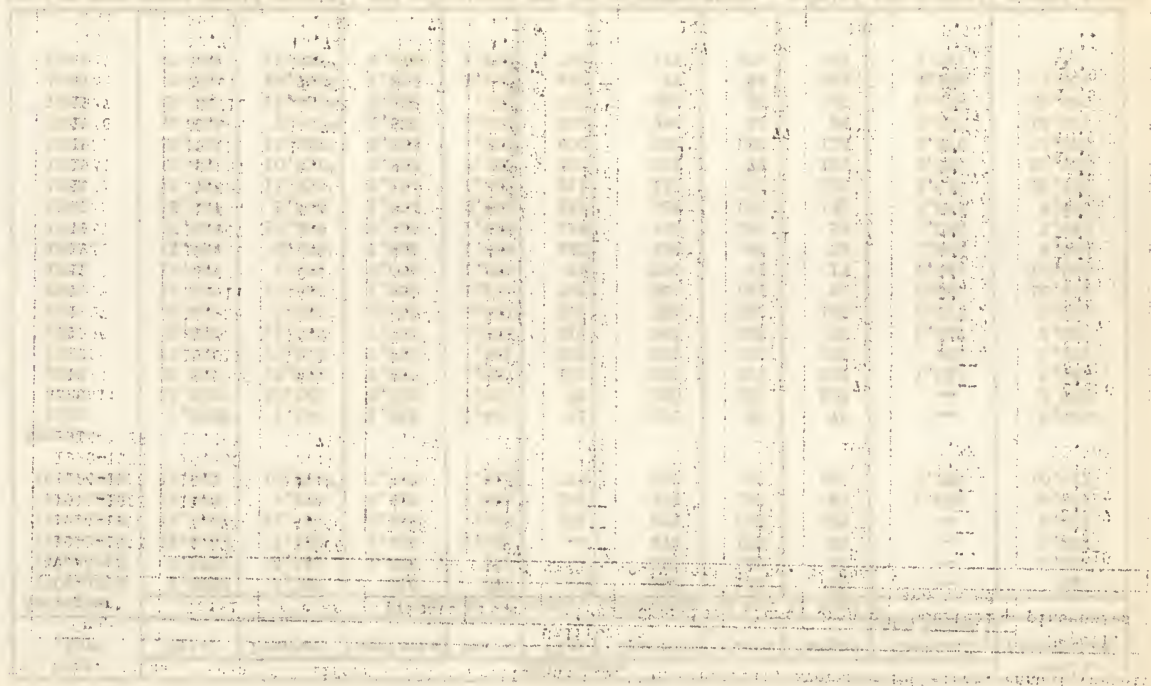

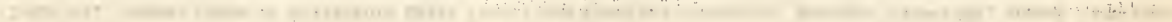


January 1942

COMPARATIVE SUMMARIES

Table 22

Canners' F.O.B. Prices for Chief Canned Fruits, 1921-1941

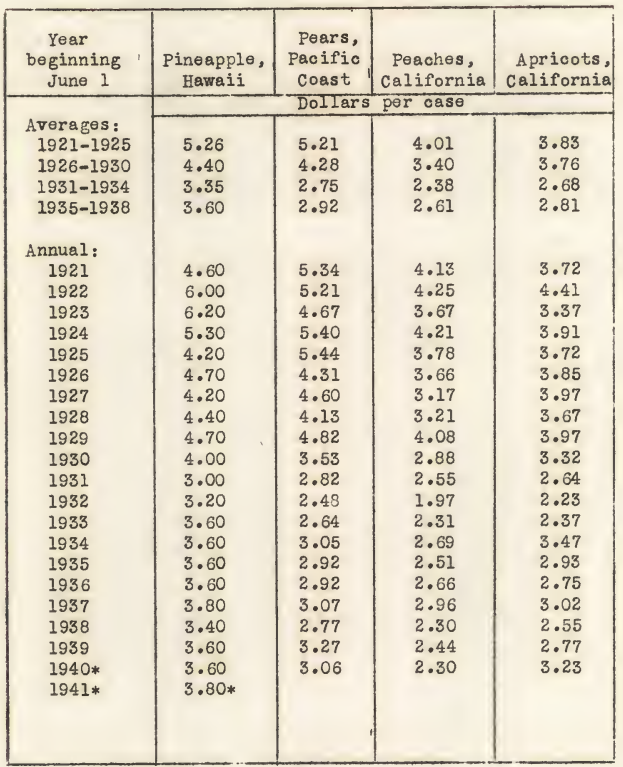

* Preliminary estimates.

Sources of data: Compiled by the Giannini Foundation of Agricultural Economios, University of California. Pineapple prices are based upon weekly price quotations of fancy grade, f. o. b. San Francisoo as reported in the california Fruit wews. Data on other canned fruits are weighted average prices of all grades and sizes of cans on an unadvertised basis and excluding trade discounts, as compiled by $H$. R. Wellman from canners' reports . 

Table 23. United States* Canned Pack of Tomato Juice and Fruit Juices, 1929-1940

\begin{tabular}{|c|c|c|c|c|c|c|c|c|c|c|c|}
\hline \multirow{3}{*}{$\begin{array}{l}\text { Crop } \\
\text { year }\end{array}$} & \multirow{3}{*}{$\begin{array}{l}\text { Grand } \\
\text { total }\end{array}$} & \multirow[b]{3}{*}{ Tomate } & \multicolumn{9}{|c|}{ Fruit juices } \\
\hline & & & \multirow[b]{2}{*}{ Total } & \multirow[b]{2}{*}{ Citrus } & \multirow[b]{2}{*}{ Pineapple } & \multirow[b]{2}{*}{ Grape } & \multirow[b]{2}{*}{ other $†$} & \multicolumn{4}{|c|}{ Citrus fruits } \\
\hline & & & & & & & & Grapefruit & Orange & Lemon & $\begin{array}{l}\text { Other } \\
\text { citrus } \neq\end{array}$ \\
\hline \multirow[b]{3}{*}{$1929-30$} & 1 & 2 & 3 & 4 & 5 & 6 & 7. & 8 & 9 & 10 & 11 \\
\hline & \multicolumn{11}{|c|}{ Thousands of cases, equivalent 24 no. 2 cans 6} \\
\hline & 1,567 & 231 & 1,336 & 230 & -9 & 1,106 & - & 192 & 38 & - & - \\
\hline $1930-31$ & 3,440 & 1,674 & 1,766 & 566 & $-\quad 11$ & 1,200 & - & 467 & 99 & - & - \\
\hline $1931-32$ & 6,393 & 4,720 & 1,673 & 378 & - & 1,295 & - & 342 & 36 & - & - \\
\hline $1932-33$ & 7,578 & 5,559 & 2,019 & 891 & -- & 1,128 & - & 780 & 111 & -- & - \\
\hline $1933-34$ & 6,495 & 4,478 & 2,017 & 1,055 & 1 & 961 & - & 712 & 343 & - & - \\
\hline $1934-35$ & 12,901 & 6,154 & 6,747 & 3,895 & 1,569 & 1,283 & - & 2,687 & 1,108 & 100 & - \\
\hline $1935-36$ & 23,209 & 11,615 & 11,594 & 4,084 & 5,783 & 1,604 & 123 & 2,472 & 1,227 & 300 & 85 \\
\hline $1936-37$ & 36,750 & 16,470 & 20,280 & 8,820 & 9,375 & 1,777 & 308 & 6,639 & 1,557 & 352 & 272 \\
\hline $1937-38$ & 40,746 & 16,880 & 23,866 & 10,947 & 8,782 & 1,950 & 2,187 & 8,935 & 1,040 & 425 & 547 \\
\hline $1938-39$ & 39,157 & 11,184 & 27,973 & 13,985 & 10,331 & 2,300 & 1,357 & 11,673 & 1,263 & 350 & 699 \\
\hline $1939-40$ & 44,454 & 13,605 & 30,849 & 16,079 & 10,846 & 3,110 & 814 & 10,969 & 3,595 & 112 & 1,403 \\
\hline $1940-41$ & 58,444 & 15,019 & 43,425 & 23,285 & 15,289 & 3,150 & 1,701 & 16,905 & 3,700 & 400 & 2,280 \\
\hline & & & & & & & & & & & \\
\hline & & & & & & & & & & & \\
\hline
\end{tabular}

* Includes imports of grapefruit juice from Puerto Rico and of pineapple juice from Hawail.

+ Includes nectars made from apricots, peaches, pears, and fresh prunes (and nectarines and others 1938-39 thru 1940-41), and juices made from dried prunes, loganberries, blackberries, cherries, raspberries, strawberries, and apples.

F Includes orange and grapefruit juice mixtures.

f. Lemon juice and other fruit juices in col. 7 are in actual, not equivalent cases.

9) Dashes indicate data are not available, but would probably be very small.

VPreliminary.

Sources of data:

Compiled by S. W. Shear, Giannini Foundation of Agricultural Economics, 1929-30 thru 1937-38 from U. S. Bur. Agr. Econ., Agricultural Outlook Charts, 1941. Fruits and Vegetables, p. 5. Washington, D. C., October 1940, and 1938-39 thru 1940-41 from U. S. Bur. Agr. Econ., Division of Statistical and Historical Research. 


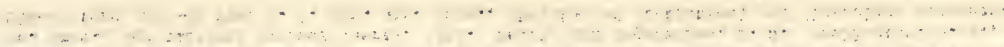

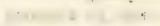

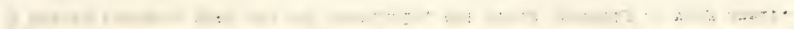

4.

$\because$.

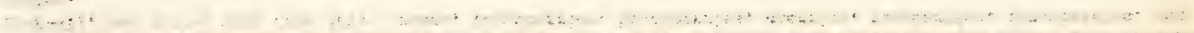

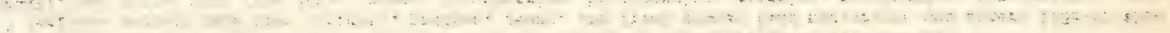

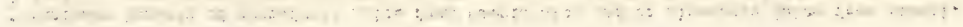

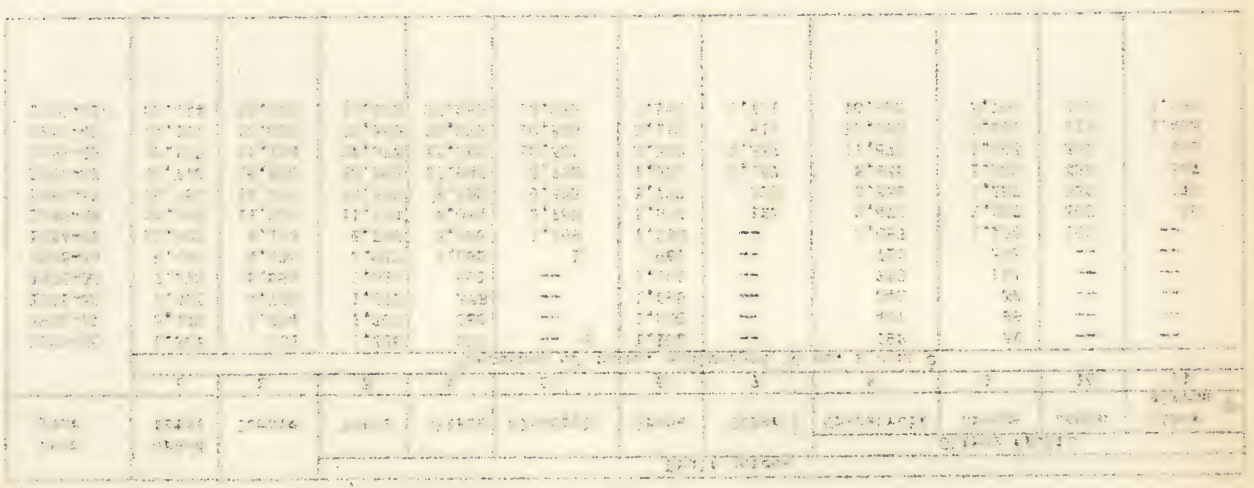

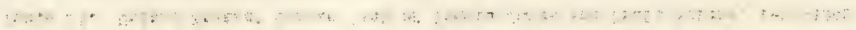


Table 24. United States Domestic Exports of Chief Fresh Fruits, 1924-1940

\begin{tabular}{|c|c|c|c|c|c|c|c|c|c|c|}
\hline \multirow{2}{*}{$\begin{array}{l}\text { Year } \\
\text { beginning } \\
\text { Julv } 1\end{array}$} & \multirow{2}{*}{$\begin{array}{r}\text { Total } \\
\text { listed }\end{array}$} & \multicolumn{4}{|c|}{ Citrus fruits } & \multicolumn{5}{|c|}{ Deciduous fruits } \\
\hline & & Total & Oranges & Grapefruit & Lemons & $\begin{array}{l}\text { Total } \\
\text { listed }\end{array}$ & AfFles & Pears & Grapes & Peaches \\
\hline \multirow{2}{*}{\multicolumn{4}{|c|}{ Averages: }} & L Cons, & $t$ dec & ed fres & weigh & & & \\
\hline & & & & & & & & & & \\
\hline $1924-1928$ & 544,409 & 139,313 & 105,007 & 24,624 & 9,682 & 405,096 & 347,429 & 32,044 & 16,982 & 8,641 \\
\hline $1929-1933$ & 608,499 & 175,288 & 126,224 & 41,008 & 8,056 & 433,211 & 358,387 & 51,839 & 17,961 &, 024 \\
\hline $1934-1938$ & 582,045 & 243,590 & 182,945 & 39,616 & 21,029 & 338,455 & 240,408 & 66,182 & 26,906 & 4,959 \\
\hline \multicolumn{11}{|l|}{ Annual: } \\
\hline 1924 & 369,984 & 100,549 & 76,895 & 17,080 & 6,574 & $-269,435$ & 230,472 & 20,726 & 10,151 & 8,086 \\
\hline 1925 & 422,293 & 102,299 & 78,855 & 15,160 & 8,284 & 319,994 & 264,384 & 35,602 & 12,134 & 7,874 \\
\hline 1926 & 725,910 & 155,366 & 116,900 & 24,520 & 13,946 & 570,544 & 510,984 & 36,938 & 15,396 & 7,226 \\
\hline 1927 & 421,690 & 141,472 & 104,580 & 28,760 & 8,132 & 280,218 & 226,296 & 25,528 & 19,410 & 8,984 \\
\hline 1928 & 782,166 & 196,881 & 147,805 & 37,600 & 11,476 & 585,285 & 505,008 & 41,424 & 27,819 & 11,034 \\
\hline 1929 & 480,681 & 169,932 & 128,590 & 34,160 & 7,182 & 310,749 & 246,672 & 31,012 & 23,079 & $9 ; 986$ \\
\hline 1930 & 785,329 & 198,504 & 139,440 & 48,880 & 10,184 & 586,825 & 488,160 & 67,335 & 24,900 & 6,430 \\
\hline 1931 & 678,817 & 181,574 & 123,690 & 48,080 & 9,804 & 497,243 & 432,720 & 45,351 & 13,806 & 5,366 \\
\hline 1932 & 566,904 & 160,465 & 118,685 & 36,080 & 5,700 & 406,439 & 330,120 & 59,994 & 14,676 & 1,649 \\
\hline 1933 & 530,763 & 165,965 & 120,715 & 37,840 & 7,410 & 364,798 & 294,264 & 55,504 & 13,344 & 1,686 \\
\hline 1934 & 461,252 & 197,172 & 143,220 & 39,360 & 14,592 & 264,080 & 193,464 & 50,318 & 17,856 & 2,442 \\
\hline 1935 & 641,166 & 261,638 & 190,610 & 43,440 & 27,588 & 379,528 & 293,760 & 62,072 & 18,676 & 5,020 \\
\hline 1936 & 407,501 & 148,676 & 107,940 & 28,120 & 12,616 & 258,825 & 162,072 & 65,659 & 23,058 & 8,036 \\
\hline 1937 & 629,349 & 259,868 & 207,620 & 34,920 & 17,328 & 369,481 & 263,016 & 67,374 & 35,014 & 4,077 \\
\hline 1938 & 770,957 & 350,597 & 265,335 & 52,240 & 33,022 & 420,360 & 289,728 & 85,486 & 39,928 & 5,218 \\
\hline 1939* & 349,394 & 191,310 & 134,890 & 33,240 & 23,180 & 158,084 & 77,184 & 46,530 & 29,980 & 4,390 \\
\hline $1940^{*}$ & 264,365 & 197,340 & 146,020 & 32,320 & 19,000 & 67,025 & 20,808 & 11,783 & 30,523 & 3,911 \\
\hline
\end{tabular}

- Preliminary.

Source of data: Compiled by S. W. Shear, Giannini Foundation of Agricultural Economics, University of California, from official data of the U. S. Bur. For and Dom. Com., as compiled largely by the Office of Foreign Agricultural Relations, U. S. Dept. Agriculture. Conversion factors used:

Oranges, 70 pounds per box; lemons, 76 pounds per box; grapefruit, 80 pounds per box; and apples, 48 pounds per bushel and 3 bushels per barrel. 


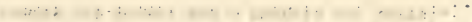

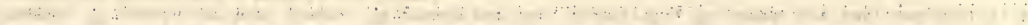
a

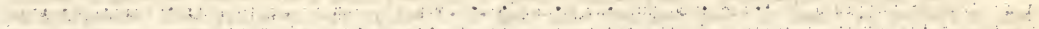

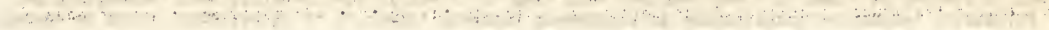

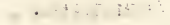

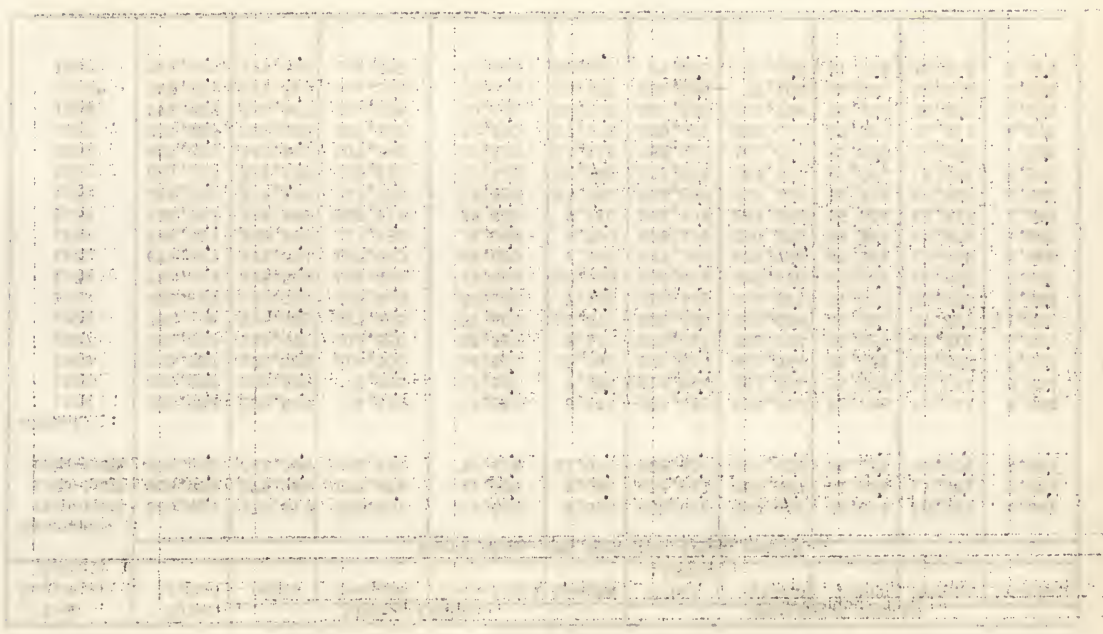

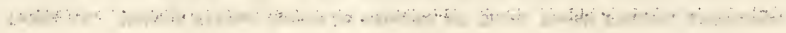


Table 25. United States Fruit Production, Exports, and Percentage of Production Exported, by Uses, Average Crop Years 1934-1938

\begin{tabular}{|c|c|c|c|c|c|c|c|c|c|c|c|c|c|c|c|}
\hline \multirow[b]{3}{*}{ Kind of fruit } & \multicolumn{3}{|c|}{ All uses } & \multicolumn{3}{|c|}{ Dried } & \multicolumn{3}{|c|}{ Canned } & \multicolumn{3}{|c|}{ Otherwise processed } & \multicolumn{3}{|c|}{ Used fresh } \\
\hline & \multirow[b]{2}{*}{$\begin{array}{c}\text { Produc } \\
\text { tion }\end{array}$} & \multicolumn{2}{|c|}{ Exports } & \multirow[b]{2}{*}{$\begin{array}{l}\text { Produc- } \\
\text { tion }\end{array}$} & \multicolumn{2}{|c|}{ Exports" } & \multirow[b]{2}{*}{$\begin{array}{l}\text { Produc- } \\
\text { tion }\end{array}$} & \multicolumn{2}{|c|}{ Exports* } & \multirow[b]{2}{*}{$\begin{array}{c}\text { Produc- } \\
\text { tion }\end{array}$} & \multicolumn{2}{|c|}{ Exports } & \multirow[b]{2}{*}{$\begin{array}{l}\text { Produc- } \\
\text { tion }\end{array}$} & \multicolumn{2}{|c|}{ Exports } \\
\hline & & $\begin{array}{l}\text { Quan- } \\
\text { tity }\end{array}$ & $\begin{array}{l}\text { Per } \\
\text { cent }\end{array}$ & & $\begin{array}{l}\text { Quan- } \\
\text { tity }\end{array}$ & $\begin{array}{l}\text { Pert } \\
\text { cent }\end{array}$ & & $\begin{array}{l}\text { Quan- } \\
\text { tity }\end{array}$ & $\begin{array}{l}\text { Per } t \\
\text { cent }\end{array}$ & & $\begin{array}{l}\text { Quan- } \\
\text { tity }\end{array}$ & $\begin{array}{l}\text { Per } \\
\text { cent }\end{array}$ & & $\begin{array}{l}\text { Quan- } \\
\text { tity }\end{array}$ & $\begin{array}{l}\text { Per } \\
\text { cent }\end{array}$ \\
\hline & $\begin{array}{c}1,000 \\
\text { fresh w }\end{array}$ & $\begin{array}{l}\text { tons } \\
\text { eight }\end{array}$ & & $\begin{array}{c}1,000 \\
\text { fresh }\end{array}$ & $\begin{array}{l}\text { tons } \\
\text { eight }\end{array}$ & & $\begin{array}{l}1,000 \\
\text { fresh we }\end{array}$ & $\begin{array}{l}\text { ons } \\
\text { ight }\end{array}$ & & $\begin{array}{l}1,000 \mathrm{t} \\
\text { fresh we }\end{array}$ & $\begin{array}{l}\text { ons } \\
\text { ight }\end{array}$ & & $\begin{array}{l}1,000 t \\
\text { fresh we }\end{array}$ & $\begin{array}{l}\text { ons } \\
\text { ight }\end{array}$ & \\
\hline Total, listed & 12,970 & 1,478 & 11.4 & 2,035 & 722 & 35.5 & 1,174 & 162 & 13.8 & 1,745 & 1 & $\neq$ & 8,016 & 593 & 7.4 \\
\hline Deciduous tree & 6,672 & 901 & 13.5 & 1,161 & 459 & 39.6 & 716 & 121 & 16.9 & 415 & 0 & 0.0 & 4,380 & 321 & 7.3 \\
\hline Grapes & 2,367 & 291 & 12.3 & 874 & 263 & 30.1 & 4 & $\neq$ & 2.4 & $1,140 \$$ & 16 & 0.1 & 349 & 27 & 7.7 \\
\hline Citrus & 3,931 & 286 & 7.3 & 0 & 0 & - & 454 & 41 & 9.0 & 190 & 0 & 0.0 & 3,287 & 245 & 7.5 \\
\hline Deciduous tree & 6,672 & 901 & 13.5 & 1,161 & 459 & 39.6 & 716 & 121 & 16.9 & 415 & 0 & 0.0 & 4,380 & 321 & 7.3 \\
\hline Apples & 3,565 & 352 & 9.9 & 158 & 97 & 61.2 & 151 & 15 & 9.8 & $397 \delta$ & \$ & \& & 2,859 & 240 & 8.4 \\
\hline Peaches & 1,238 & 72 & 5.8 & 139 & 24 & $17 \cdot 4$ & 271 & 43 & 15.9 & 0 & 0 & - & 828 & 5 & 0.6 \\
\hline Pears & 679 & 139 & 20.4 & 32 & 25 & 76.3 & 161 & 48 & 29.8 & 0 & 0 & - & 486 & 66 & 13.6 \\
\hline Prunes & 611 & 243 & 39.8 & 611 & 243 & 39.8 & ǵ & $\dot{9}$ & g & 0 & 0 & - & 0 & 0 & - \\
\hline Apricots & 225 & 85 & 38.0 & 144 & 70 & 49.1 & 55 & 13 & 23.6 & 0 & 0 & - & 26 & $2 \xi$ & 7.2 \\
\hline Plums & 136 & 7 & 5.4 & 0 & 0 & - & 28 & fọ & 1.4 & 0 & 0 & - & 108 & $7 \xi$ & 6.5 \\
\hline Cherries & 129 & 3 & 1.8 & $\xi$ & 0 & - & 43 & 2 & 4.0 & $18 \xi$ & 0 & 0.0 & 68 & 15 & 0.9 \\
\hline Figs & 89 & 이 & 0.0 & 77 & 0 & 0.0 & 7 & 0 & 0.0 & 0 & 0 & - & 5 & 0 & 0.0 \\
\hline Grapes, total & 2,367 & 291 & 12.3 & 874 & 263 & 30.1 & 4 & $\neq$ & 2.4 & $1,140 \$$ & $1 \xi$ & 0.1 & 349 & 27 & 7.7 \\
\hline Citrus, total & 3,931 & 286 & 7.3 & 0 & 0 & - & 454 & 41 & 9.0 & 190 & 0 & 0.0 & 3,287 & 245 & 7.5 \\
\hline Oranges & $2,468 \%$ & 183 & 7.4 & 0 & 0 & - & $54 \S$ & 0 & 0.0 & $123 \oint^{\circ}$ & 0 & 0.0 & $2,291 \hat{\rho}$ & 183 & 8.0 \\
\hline Grapefruit & $1,110 \$$ & 80 & 7.2 & 0 & 0 & - & $389 \oint$ & 41 & 10.5 & $4 \delta$ & 0 & 0.0 & $717 \delta$ & 39 & 5.4 \\
\hline Lemons & $353^{\circ}$ & 23 & 6.4 & 0 & 0 & - & $11 \xi$ & 0 & 0.0 & 63 क & 0 & 0.0 & $279^{\circ}$ & 23 & 8.1 \\
\hline
\end{tabular}

* Includes exports of dried fruits in salad and of canned fruits in salad.

+ Percentages computed from data to the nearest 100 short tons.

f Less than 500 short tons or less than 0.05 per cent.

\$ Numerous detailed footnotes on original table omitted for lack of space.

Source of data: Compiled by S. W. Shear, Giannini Foundation, largely upon the besis of official data but includes numerous conversions and estimates involving assumptions by the compiler. For annual data comparing production and exports of fruits, see U. S. Dept. Agriculture, Agricultural Statistics, 1941. 


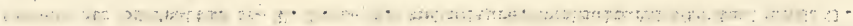

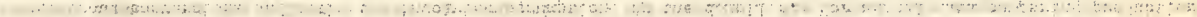

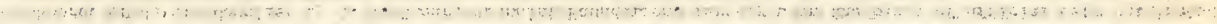

.

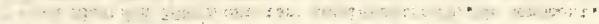

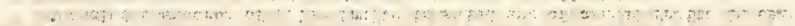

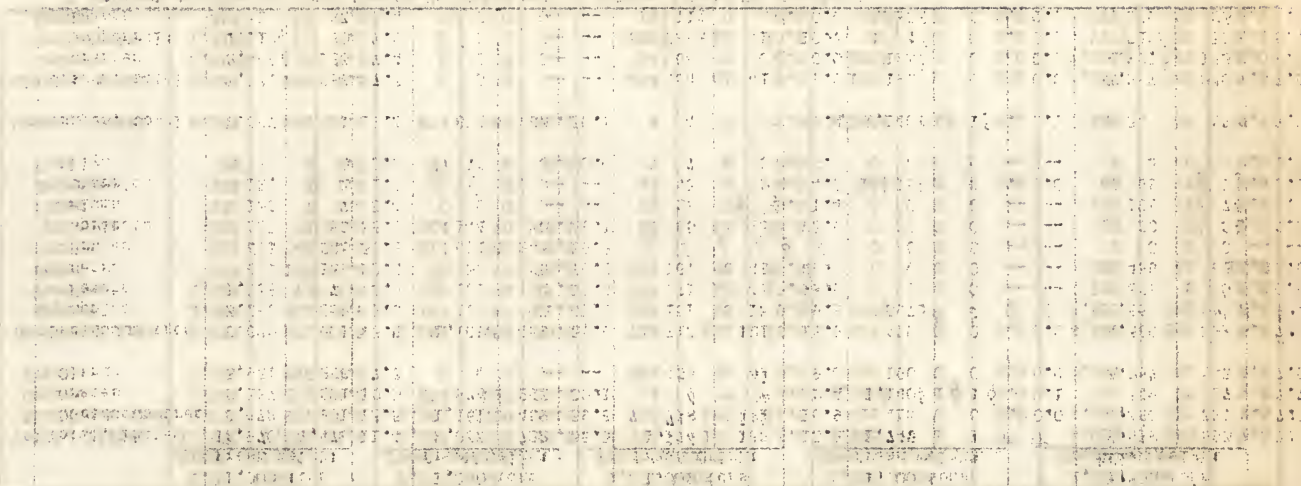

1 .

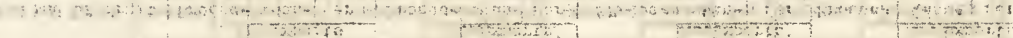

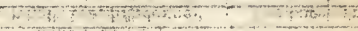

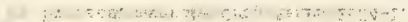

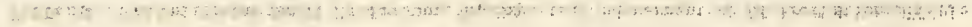


Table 26. California Fruit Production, Exports, and Percentage of Production Exported, by Uses Average Crop Years 1934-1938

\begin{tabular}{|c|c|c|c|c|c|c|c|c|c|c|c|c|c|c|c|}
\hline \multirow[b]{3}{*}{ Kind of fruit } & \multicolumn{3}{|c|}{ All uses } & \multicolumn{3}{|c|}{ Dried } & \multicolumn{3}{|c|}{ Canned } & \multicolumn{3}{|c|}{ Otherwise processed } & \multicolumn{3}{|c|}{ Used fresh } \\
\hline & \multirow[b]{2}{*}{$\begin{array}{c}\text { Produc- } \\
\text { tion }\end{array}$} & \multicolumn{2}{|c|}{ Exports } & \multirow[b]{2}{*}{$\begin{array}{c}\text { Produc- } \\
\text { tion }\end{array}$} & \multicolumn{2}{|c|}{ Exports* } & \multirow[b]{2}{*}{$\begin{array}{l}\text { Produc- } \\
\text { tion }\end{array}$} & \multicolumn{2}{|c|}{ Exports* } & \multirow[b]{2}{*}{$\begin{array}{c}\text { Produc- } \\
\text { tion }\end{array}$} & \multicolumn{2}{|c|}{ Exports } & \multirow[b]{2}{*}{$\begin{array}{l}\text { Produc- } \\
\text { tion }\end{array}$} & \multicolumn{2}{|c|}{ Exports } \\
\hline & & $\begin{array}{l}\text { Guan- } \\
\text { tity }\end{array}$ & $\begin{array}{l}\text { Perf } \\
\text { cent }\end{array}$ & & $\begin{array}{l}\text { Quan- } \\
\text { tity }\end{array}$ & $\begin{array}{l}\text { Pert } \\
\text { cent }\end{array}$ & & $\begin{array}{l}\text { Quan- } \\
\text { tity }\end{array}$ & $\begin{array}{l}\text { Per } \\
\text { cent }\end{array}$ & & $\begin{array}{l}\text { Quan- } \\
\text { tity }\end{array}$ & $\begin{array}{l}\text { Per } t \\
\text { cent }\end{array}$ & & $\begin{array}{l}\text { Quan- } \\
\text { tity }\end{array}$ & $\begin{array}{l}\text { Pert } \\
\text { cent }\end{array}$ \\
\hline & $\begin{array}{r}1,000 \\
\text { fresh } w\end{array}$ & $\begin{array}{l}\text { tons } \\
\text { eight }\end{array}$ & & $\begin{array}{l}1,000 \\
\text { fresh we }\end{array}$ & $\begin{array}{l}\text { tons } \\
\text { eight }\end{array}$ & & $\begin{array}{l}1,000 t \\
\text { fresh we }\end{array}$ & $\begin{array}{l}\text { ons } \\
\text { ight }\end{array}$ & & $\begin{array}{l}1,000 \text { t } \\
\text { fresh we }\end{array}$ & $\begin{array}{l}\text { ons } \\
\text { ight }\end{array}$ & & $\begin{array}{l}1,000 \text { t } \\
\text { fresh we }\end{array}$ & $\begin{array}{l}\text { ons } \\
\text { ight }\end{array}$ & \\
\hline Total, listed & 5,667 & 1,014 & 17.9 & 1,869 & 647 & 34.6 & 435 & 78 & 17.9 & 1,165 & 1 & 0.1 & 2,198 & 288 & 13.1 \\
\hline Deciduous tree & 1,823 & 517 & 28,4 & 995 & 384 & 38.6 & 396 & 78 & $19 \cdot 6$ & 21 & 0 & 0.0 & 411 & 55 & 13.4 \\
\hline Grapes & 2,119 & 291 & 13.7 & 874 & 263 & 30.1 & 4 & $\neq$ & 2.4 & 9549 & $1 \oint$ & 0.1 & 287 & 27 & 9.4 \\
\hline Citrus & 1,725 & 206 & 11.9 & 0 & 0 & - & $35 \xi$ & 0 & 0.0 & $190 \$$ & 0 & 0.0 & 1,500 & 206 & 13.7 \\
\hline Deciduous tree & 1,823 & 517 & 28.4 & 995 & 384 & 38.6 & 396 & 78 & 19.6 & 21 & 0 & 0.0 & $\dot{4} 11$ & 55 & 13.4 \\
\hline Apples & 207 & 61 & 29.3 & 72 & 48 & 65.3 & 1 & 8 & $\$$ & $15 \phi$ & 4 & 9 & $119 \oint$ & 13 & 10.9 \\
\hline Peaches & 481 & 70 & 14.5 & 139 & 24 & 17.4 & 265 & 43 & 16.2 & 0 & 0 & - & 77 & 3 & 3.2 \\
\hline Pears & 220 & 77 & 35.1 & 32 & 25 & 76.3 & 66 & 2] & 32.3 & 0 & 0 & -- & 122 & 31 & 25.8 \\
\hline Prunes & 531 & 217 & 41.0 & 531 & 217 & 41.0 & $\oint$ & $\delta$ & $\oint$ & 0 & 0 & -- & 0 & 0 & - \\
\hline Apricots & 216 & 85 & 39.6 & 144 & 70 & 49.1 & 54 & 13 & 23.9 & 0 & 0 & -- & .18 & $2 \xi$ & 10.6 \\
\hline Plums & 61 & $6 \hat{\wp}$ & 9.4 & 0 & 0 & -- & 2 & 9 & 9 & 0 & 0 & - & 59 & $6 \%$ & 9.7 \\
\hline Cherries & 20 & 1 & 4.9 & of & 0 & -- & 3 & 1 & 17.6 & $6 \oint$ & 9 & of & 11 & $\neq \phi$ & 3.6 \\
\hline Figs & 87 & 0 & 0.0 & 77 & 0 & 0.0 & 5 & 0 & 0.0 & 0 & 0 & $\therefore$ & 5 & 0 & 0.0 \\
\hline Grapes, total & 2,119 & 291 & 13.7 & 874 & 263 & 30.1 & 4 & キ & 2.4 & $954 \oint$ & $1\}$ & 0.1 & 287 & 27 & 9.4 \\
\hline Citrus, total & 1,725 & 206 & 11.9 & 0 & 0 & - & $35 \$$ & 0 & 0.0 & $190 \$$ & 0 & 0 & 1,500 & 206 & 13.7 \\
\hline Oranges & 1,315 & 183 & 13.9 & 0 & 0 & - & $24 \$$ & 0 & 0.0 & 1239 & 0 & 0 & 1,168 & 183 & 15.7 \\
\hline Grapefruit & 57 & $\Phi$ & 9 & 0 & 0 & - & 9 & 9 & - & 4 & 0 & 0 & 53 & 8 & $\Phi$ \\
\hline Lemons & 353 & 23 & 6.4 & 0 & 0 & - & If 9 & $\delta$ & 0.0 & $63 \dot{9}$ & 0 & 0 & 279 & 23 & 8.1 \\
\hline
\end{tabular}

* Includes exports of dried fruits in salad and of canned fruits in salad.

T Percentages computed from data to the nearest 100 short tons.

f Less than 500 short tons.

$\$$ Numerous detailed footnotes on original table omitted for lack of space. 9 Assumed to be zero.

Source of data: Compiled by S. ". Shear, Giannini Foundation, largely upon the basis of official data but includes numerous conversions and estimates involving assumotions by the compiler. 


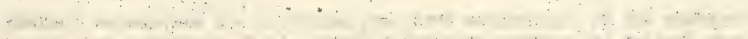

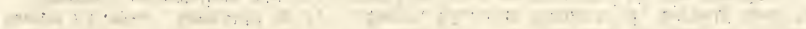

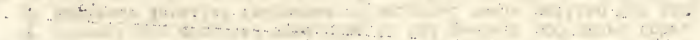

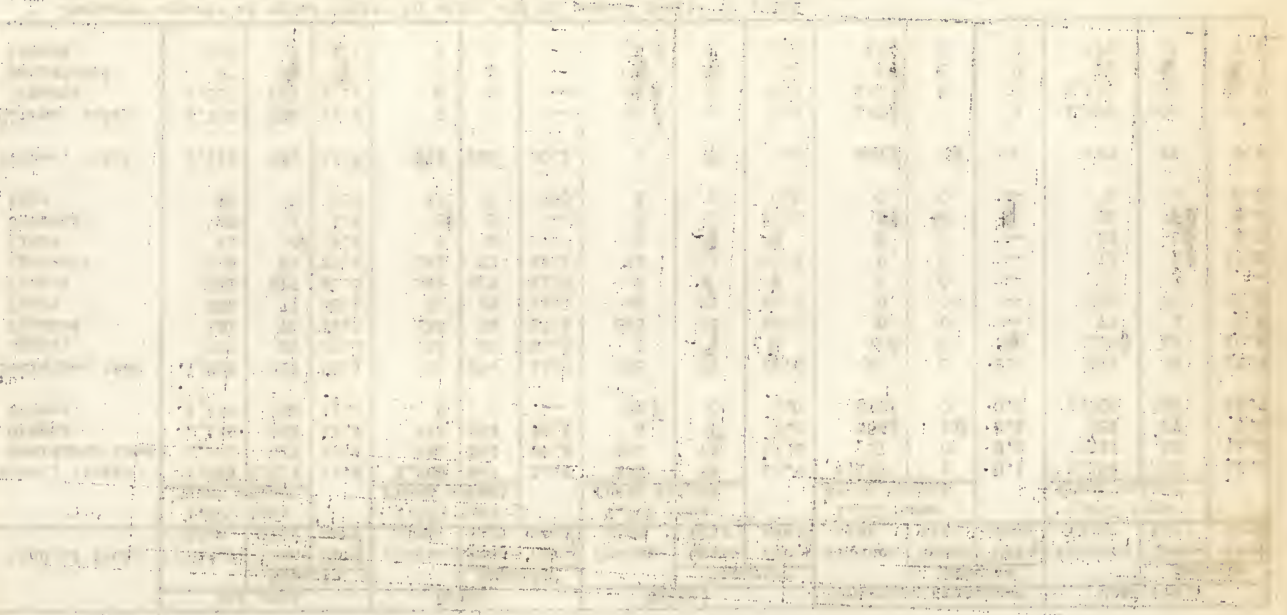

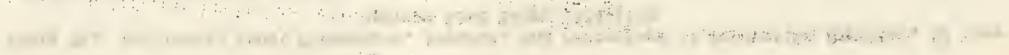


Table 27. United States Fruit Production, Exports to Europe, and Percentage of Production Exported to Europe, by Uses, Average Crop Years 1934-1938

\begin{tabular}{|c|c|c|c|c|c|c|c|c|c|c|c|c|c|c|c|}
\hline \multirow{3}{*}{$\begin{array}{l}\text { Kind of } \\
\text { fruit }\end{array}$} & \multicolumn{3}{|c|}{ All uses } & \multicolumn{3}{|c|}{ Dried } & \multicolumn{3}{|c|}{ Canned } & \multicolumn{3}{|c|}{ Otherwise processed } & \multicolumn{3}{|c|}{ Used fresh } \\
\hline & \multirow[b]{2}{*}{$\begin{array}{c}\text { Produc- } \\
\text { tion }\end{array}$} & \multicolumn{2}{|c|}{ Exports } & \multirow[b]{2}{*}{$\begin{array}{l}\text { Produc- } \\
\text { tion }\end{array}$} & \multicolumn{2}{|c|}{ Exports* } & \multirow[b]{2}{*}{$\begin{array}{c}\text { Produc- } \\
\text { tion }\end{array}$} & \multicolumn{2}{|c|}{ Exports* } & \multirow[b]{2}{*}{$\begin{array}{l}\text { Produc- } \\
\text { tion }\end{array}$} & \multicolumn{2}{|c|}{ Exports } & \multirow[b]{2}{*}{$\begin{array}{c}\begin{array}{c}\text { Froduc } \\
\text { tion }\end{array} \\
\end{array}$} & \multicolumn{2}{|c|}{ Exports } \\
\hline & & $\begin{array}{l}\text { Quan- } \\
\text { tity }\end{array}$ & $\begin{array}{l}\text { Pert } \\
\text { cent }\end{array}$ & & $\begin{array}{l}\text { Quan- } \\
\text { tity }\end{array}$ & $\begin{array}{l}\text { Per } \\
\text { cent }\end{array}$ & & $\begin{array}{l}\text { Quan- } \\
\text { tity }\end{array}$ & $\begin{array}{l}\text { Per } \\
\text { cent }\end{array}$ & & $\begin{array}{l}\text { Quan- } \\
\text { tity }\end{array}$ & $\begin{array}{l}\text { Per } \\
\text { cent }\end{array}$ & & $\begin{array}{l}\text { Quan- } \\
\text { tity }\end{array}$ & $\begin{array}{l}\text { Pert } \\
\text { cent }\end{array}$ \\
\hline & $\begin{array}{l}1,000 \\
\text { fresh }\end{array}$ & $\begin{array}{l}\text { tons } \\
\text { eight }\end{array}$ & & $\begin{array}{r}1,000 \\
\text { fresh }\end{array}$ & $\begin{array}{l}\text { tons } \\
\text { veight }\end{array}$ & & $\begin{array}{c}1,000 \\
\text { fresh w }\end{array}$ & $\begin{array}{l}\text { tons } \\
\text { eight }\end{array}$ & & $\begin{array}{l}1,000 \\
\text { fresh }\end{array}$ & $\begin{array}{l}\text { tons } \\
\text { eight }\end{array}$ & & $\begin{array}{l}1,000 \\
\text { fresh }\end{array}$ & $\begin{array}{l}\text { tons } \\
\text { veight } \\
\end{array}$ & \\
\hline Total & 12,970 & 1,129 & 8.7 & 2,035 & 610 & 29.9 & 1,174 & 155 & 13.2 & 1,745 & 0 & 0.0 & 8,016 & 364 & 4.5 \\
\hline Deciduous tree & 6,672 & 768 & 11.5 & 1,161 & 397 & 34.2 & 716 & 115 & 16.1 & 415 & 0 & 0.0 & 4,380 & 256 & 5.8 \\
\hline Grapes & 2,367 & 221 & 9.3 & 874 & 213 & 24.3 & 4 & $\neq$ & 2.4 & 1,1409 & 9 & 9 & 349 & 8 & 2.4 \\
\hline Citrus & 3,931 & 140 & 3.6 & 0 & 0 & - & 454 & 40 & 8.8 & 190 & 0 & 0.0 & 3,287 & 100 & 3.0 \\
\hline Deciduous tree & 6,672 & 768 & 11.5 & 1,161 & 397 & 34.2 & 716 & 115 & 16.1 & 415 & 0 & 0.0 & 4,380 & 256 & 5.8 \\
\hline Apples & 3,565 & 313 & 8.8 & 158 & 93 & 58.6 & 151 & 14 & 9.5 & $397 \oint$ & § & 0.0 & 2,859 & 206 & 7.2 \\
\hline Peaches & 1,238 & 58 & 4.7 & 139 & 17 & 12.3 & 271 & 41 & 15.0 & 0 & 0 & - & 828 & $\neq$ & $\neq$ \\
\hline Pears & 679 & 119 & 17.5 & 32 & 22 & 70.0 & 161 & 47 & 28.7 & 0 & 0 & -- & 486 & 50 & 10.3 \\
\hline Prunes & 611 & 201 & 32.8 & 611 & 201 & $32 \cdot 8$ & & $\S$ & § & 0 & 0 & - & 0 & 0 & -- \\
\hline Apricots & 225 & 76 & 34.0 & 144 & 64 & 44.6 & 55 & 12 & 22.7 & 0 & 0 & -- & 26 & 91 & 9 \\
\hline Plums & 136 & $\neq$ & 0.1 & 0 & 0 & - & 28 & $\neq$ & 0.7 & 0 & 0 & -- & 108 & 9 & $\$$ \\
\hline Cherries & 129 & 1 & 0.9 & $\oint$ & 0 & - & 43 & 1 & 2.8 & $18 \phi$ & 0 & 0.0 & 68 & 9 & 9 \\
\hline Figs & 89 & 0 & 0.0 & 77 & 0 & 0.0 & 7 & 0 & 0.0 & 0 & 0 & -- & 5 & 0 & 0.0 \\
\hline Grapes, total & 2,367 & 221 & 9.3 & 874 & 213 & 24.3 & 4 & $\neq$ & 2.4 & $1,140 \$$ & 9 & 4 & 349 & 8 & 2.4 \\
\hline Citrus, total & 3,931 & 140 & 3.6 & 0 & 0 & - & 454 & 40 & 8.8 & 190 & 0 & 0.0 & 3,287 & 100 & 3.0 \\
\hline Oranges & $2,468 \oint$ & 78 & 3.2 & 0 & 0 & - & $54 \%$ & 0 & 0.0 & 1239 & 0 & 0.0 & 2,2916 & 78 & 3.4 \\
\hline Grapefruit & $1,110 \emptyset$ & 54 & 4.8 & 0 & 0 & - & $389 \varnothing$ & 40 & 10.3 & $4 \oint$ & 0 & 0.0 & 7179 & 14 & 1.9 \\
\hline Lemons & 353 & 8 & 2.3 & 0 & 0 & - & $11 \oint$ & 0 & 0.0 & $63 \delta$ & 0 & 0.0 & 279 & 8 & 2.9 \\
\hline
\end{tabular}

* Includes exports of dried fruits in salad and of canned fruits in salad. TPercentages computed from data to the nearest 100 tons. $\neq$ Less than 500 tons or less than 0.05 per cent. $\oint$ Numerous detailed footnotes on oríginal table-omitted for lack of space. 9 Assumed to be zero.

Source of data:

Compiled by S. W. Shear, Giannini Foundation, largely upon the basis of official data but includes numerous conversions and estimates involving assumptions by the compiler. 
- $\quad \cdots+\cdots$

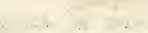

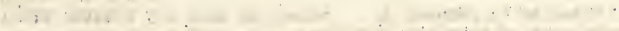

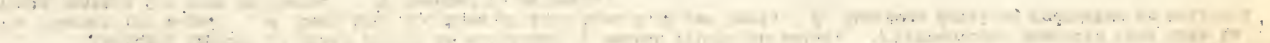

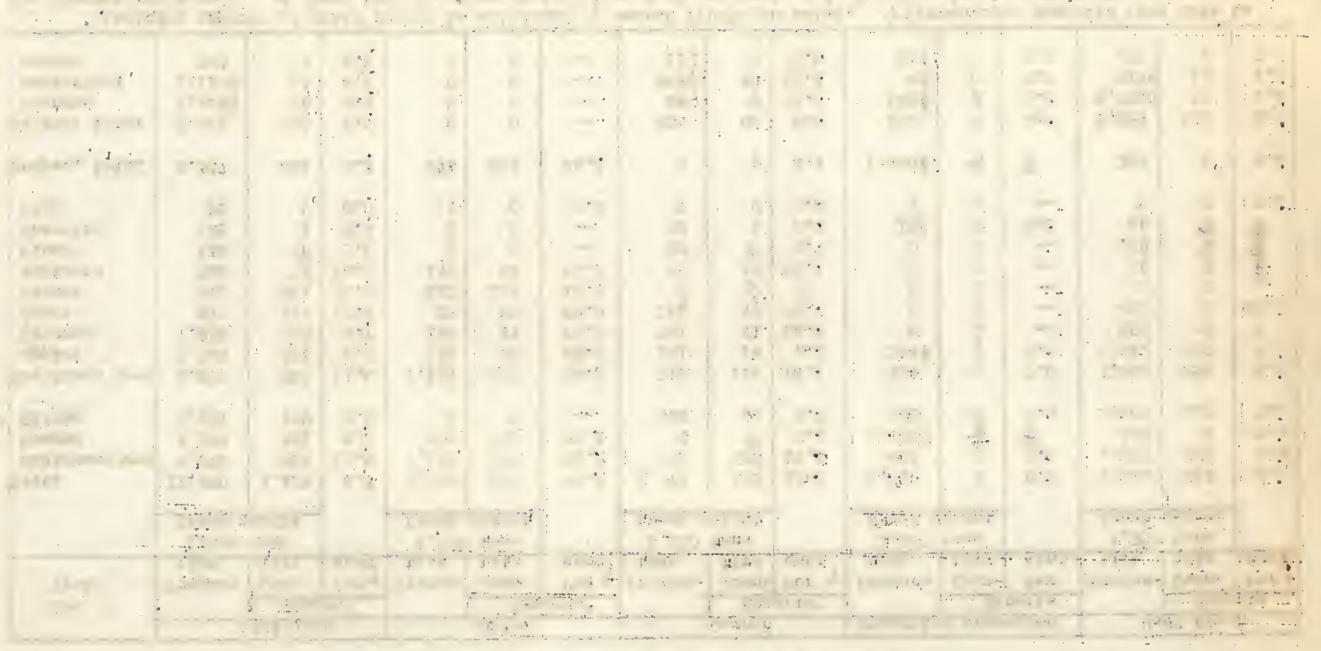

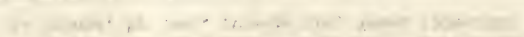

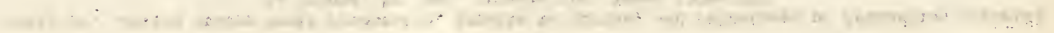


Table 28. California Fruit Production, Exports to Europe, and Percentage of Production Exported to Europe by Uses, Average Crop Years 1934-1938

\begin{tabular}{|c|c|c|c|c|c|c|c|c|c|c|c|c|c|c|c|}
\hline \multirow[b]{3}{*}{ Kind of fruit } & \multicolumn{3}{|c|}{ All uses } & \multicolumn{3}{|c|}{ Dried } & \multicolumn{3}{|c|}{ Canned } & \multicolumn{3}{|c|}{ Otherwise processed } & \multicolumn{3}{|c|}{ Used fresh } \\
\hline & \multirow[b]{2}{*}{$\begin{array}{c}\text { Produc- } \\
\text { tion }\end{array}$} & \multicolumn{2}{|c|}{ Exports } & \multirow[b]{2}{*}{$\begin{array}{c}\text { Produc- } \\
\text { tion }\end{array}$} & \multicolumn{2}{|c|}{ Exports : } & \multirow[b]{2}{*}{$\begin{array}{l}\text { Produc- } \\
\text { tion }\end{array}$} & \multicolumn{2}{|c|}{ Exports* } & \multirow[b]{2}{*}{$\begin{array}{c}\text { Produc- } \\
\text { tion }\end{array}$} & \multicolumn{2}{|c|}{ Exports } & \multirow[b]{2}{*}{$\begin{array}{l}\text { Produc- } \\
\text { tion }\end{array}$} & \multicolumn{2}{|c|}{ Exports } \\
\hline & & $\begin{array}{l}\text { Quan- } \\
\text { tity }\end{array}$ & $\begin{array}{l}\text { Per } t \\
\text { cent }\end{array}$ & & $\begin{array}{l}\text { Quan- } \\
\text { tity }\end{array}$ & $\begin{array}{l}\text { Pert } \\
\text { cent }\end{array}$ & & $\begin{array}{l}\text { Quan- } \\
\text { tity }\end{array}$ & $\begin{array}{l}\text { Per } \\
\text { cent }\end{array}$ & & $\begin{array}{l}\text { Quan- } \\
\text { tity }\end{array}$ & $\begin{array}{l}\text { Per } \\
\text { cent }\end{array}$ & & $\begin{array}{l}\text { Quan- } \\
\text { tity }\end{array}$ & $\begin{array}{l}\text { Per } \\
\text { cent }\end{array}$. \\
\hline & $\begin{array}{l}1,000 \\
\text { fresh }\end{array}$ & $\begin{array}{l}\text { tons } \\
\text { eight }\end{array}$ & & $\begin{array}{l}1,000 \\
\text { fresh } w\end{array}$ & $\begin{array}{l}\text { tons } \\
\text { eight }\end{array}$ & & $\begin{array}{l}1,000 \\
\text { fresh we }\end{array}$ & $\begin{array}{l}\text { ons } \\
\text { ight }\end{array}$ & & $\begin{array}{l}1,000 t \\
\text { fresh we }\end{array}$ & $\begin{array}{l}\text { ons } \\
\text { ight }\end{array}$ & & $\begin{array}{l}1,000 t \\
\text { fresh we }\end{array}$ & $\begin{array}{l}\text { ons } \\
\text { ight }\end{array}$ & \\
\hline Total & 5,667 & 744 & 13.1 & 1,869 & 541 & 28.9 & 435 & 74 & 17.0 & 1,165 & 0 & 0.0 & 2,198 & 129 & 5.9 \\
\hline Deciduous tree & 1,823 & 437 & 24.0 & 995 & 328 & 33.0 & 396 & 74 & 18.7 & 21 & 0 & 0.0 & 411 & 35 & 8.5 \\
\hline Grapes & 2,119 & 221 & 10.4 & 874 & 213 & 24.3 & 4 & $\neq$ & 2.4 & $954 \hat{q}$ & 9 & 9 & 287 & 8 & 2.9 \\
\hline Citrus & 1,725 & 86 & 5.0 & 0 & 0 & - & $35 \oint$ & 0 & 0.0 & $190 \varphi^{\circ}$ & 0 & 0.0 & 1,500 & 86 & 5.8 \\
\hline Deciduous tree & 1,823 & 437 & 24.0 & 995 & 328 & 33.0 & 396 & 74 & 18.7 & 21 & 0 & 0.0 & 411 & 35 & 8.5 \\
\hline Apples & 207 & 57 & 27.4 & 72 & 46 & 62.6 & 1 & 9 & 4 & $15 \hat{\beta}$ & 9 & 9 & $119 \oint$ & 11 & 9.3 \\
\hline Peaches & 481 & 58 & 12.0 & 139 & 17 & 12.3 & 265 & 41 & 15.4 & 0 & 0 & - & 77 & $\neq$ & $\neq$ \\
\hline Pears & 220 & 67 & 30.3 & 32 & 22 & 70.0 & 66 & 21 & 31.1 & 0 & 0 & - & 122 & 24 & 19.6 \\
\hline Prunes & 531 & 179 & 33.8 & 531 & 179 & 33.8 & $\oint$ & $\oint$ & 6 & 0 & 0 & -- & 0 & 0 & - \\
\hline Apricots & 216 & 76 & 35.5 & 144 & 64 & 44.6 & 54 & 12 & 23.0 & 0 & 0 & - & 18 & 9 & 9 \\
\hline Plums & 61 & 9 & 9 & 0 & 0 & - & 2 & 9 & 9 & 0 & 0 & - & 59 & 4 & की \\
\hline Cherries & 20 & $\neq$ & 2.0 & $0 \oint$ & 0 & - & 3 & $\neq$ & 11.8 & 66 & $\Phi$ & 7 & 11 & & \\
\hline Figs & 87 & 0 & 0.0 & 77 & 0 & 0.0 & 5 & 0 & 0.0 & 0 & 0 & - & 5 & 0 & 0.0 \\
\hline Grapes, total & 2,119 & 221 & 10.4 & 874 & 213 & 24.3 & 4 & $\neq$ & 2.4 & $954 \phi$ & $\mathbb{T}$ & 9 & 287 & 8 & 2.9 \\
\hline Citrus, total & 1,725 & 86 & 5.0 & 0 & 0 & - & $35 \xi$ & 0 & 0.0 & $190 \varnothing$ & 0 & 0.0 & 1,500 & 86 & 5.8 \\
\hline Oranges & 1,315 & 78 & 6.0 & 0 & 0 & -. & $24 \zeta$ & 0 & 0.0 & $123 \xi$ & 0 & 0.0 & 1,168 & 78 & 6.7 \\
\hline Grapefruit & 57 & 9 & 9 & 0 & 0 & - & 9 & प & -- & 4 ó & 0 & 0.0 & 53 & 9 & 9 \\
\hline Lemons & 353 & 8 & 2.3 & 0 & 0 & - & $11 \oint$ & 0 & 0.0 & 636 & 0 & 0.0 & 279 & $8^{3}$ & 2.9 \\
\hline
\end{tabular}

" Includes exports of dried fruits in salad and of canned fruits in salad.

+ Fercentages computed from data to the nearest 100 tons.

+ Less than 500 tons or less than 0.05 per cent.

\$ Numerous detriled footnotes on original table omitted for lack.of space. 9 Assumed to be zero.

Source of data: Compiled by S. W. Shear, Giannini Foundation, largely upon the basis of official data but includes numerous conversions and estimates involving assumptions by the compiler. 


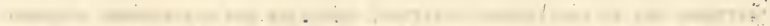

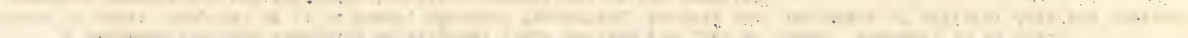

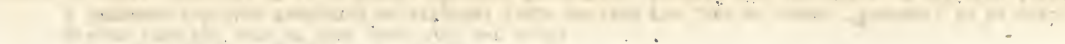

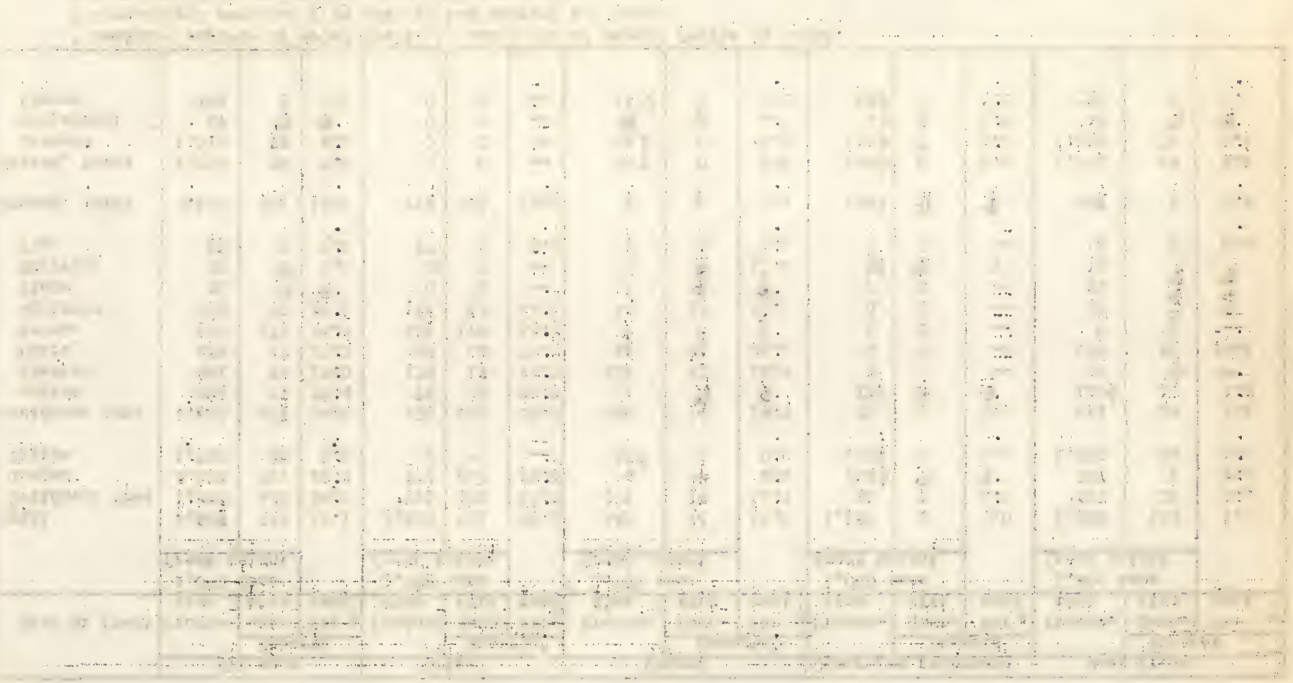

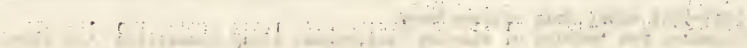


Table 29. Percentage by Uses of Harvested Production of United States and California Fruits, Average Crop Years 1934-1938

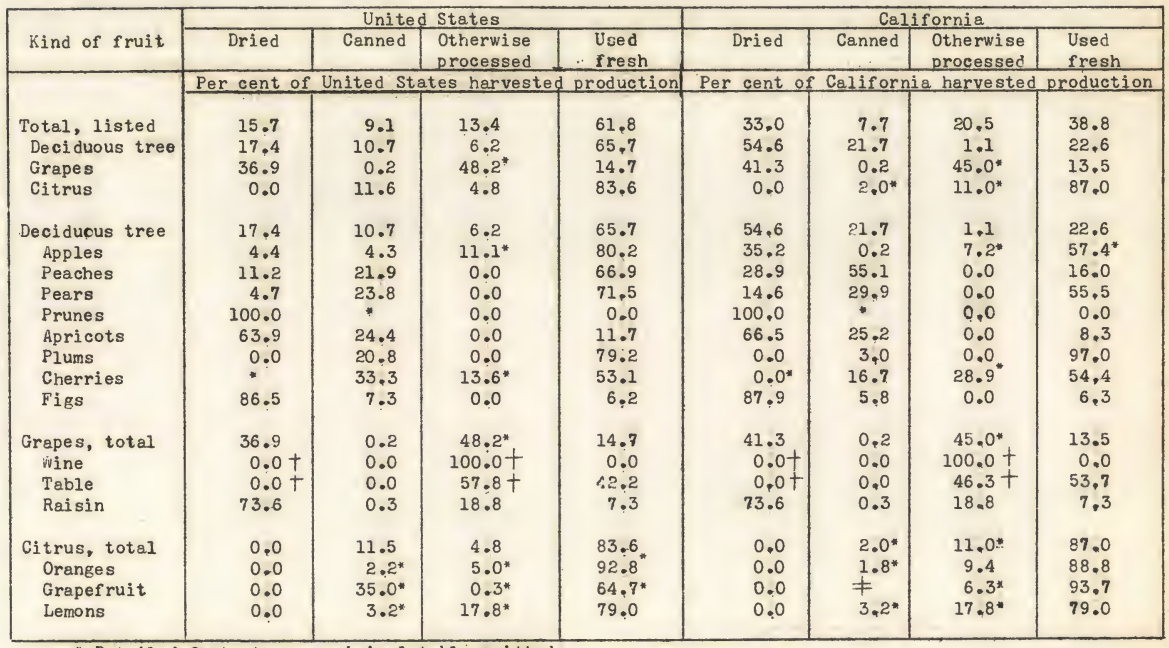

Detailed footnotes on original table cmitted.

+ Dried table and wine varieties included in "otherwise processed" as mostly used for juice ultimately. $\neq$ Assumed to be zero.

Source of data: Compiled by S. W. Shear, Giannini Foundation, based on original data to the nearest 100 tons from which tables 25 and 26 were compiled, except utilization of grapes by varieties based on data on Grapes, tables 2 and $6-9$. 


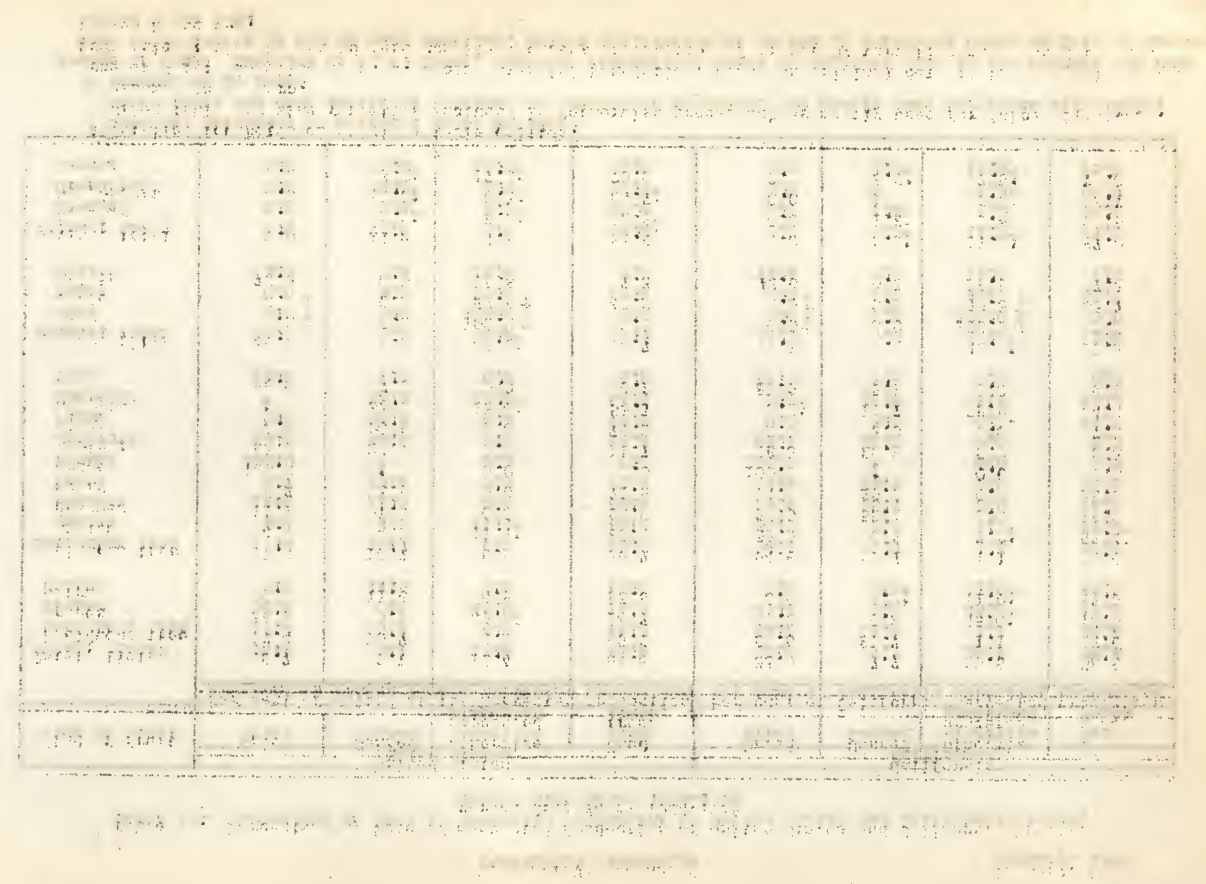


Table 30. United States Domestic Exports* of Fruits to Foreign Countries

\begin{tabular}{|c|c|c|c|c|c|c|}
\hline \multirow{3}{*}{ Fruit } & \multicolumn{6}{|c|}{ Iwelve months, beginnin, July 1} \\
\hline & \multirow{2}{*}{1940} & \multirow{2}{*}{1939} & \multirow{2}{*}{1938} & \multicolumn{3}{|c|}{ Five-year averages } \\
\hline & & & & $1934-1938$ & $1929-1933$ & $1924-1928$ \\
\hline \multicolumn{7}{|c|}{ fresh weight } \\
\hline Total 1 isted & $270-774$ & 358.434 & & & & 550,348 \\
\hline Citrus, total & 197,340 & $\begin{array}{l}300,434 \\
191,310\end{array}$ & 350,597 & $\begin{array}{l}587,401 \\
243,590\end{array}$ & $\begin{array}{l}613,871 \\
175,288\end{array}$ & $\begin{array}{l}500,348 \\
139,313\end{array}$ \\
\hline Oranges & 146,020 & 134,890 & 265,335 & 182,945 & 126,224 & 105,007 \\
\hline Grapefruit & 32,320 & 33,240 & 52,240 & 39,616 & 41,008 & 24,624 \\
\hline Lemons & 19,000 & 23,180 & 33,022 & 21,029 & 8,056 & 9,682 \\
\hline Deciduous, total & 73,434 & 167,124 & 426,336 & 343,811 & 438,583 & 411,035 \\
\hline Deciduous tree & 42,911 & 137,144 & 386,408 & 316,905 & 420,622 & 394,053 \\
\hline Apples & 20,808 & 77,172 & 289,702 & 240,407 & 358,387 & 347,429 \\
\hline Dears & 11,783 & 46,530 & 85,486 & 66,182 & 51,839 & 32,044 \\
\hline Peaches & 3,911 & 4,760 & 5,384 & 4,928 & 5,024 & 8,641 \\
\hline Plums & 4,576 & $5,856+$ & $3,865+$ & $3,475+$ & $4,333+$ & $4,695+$ \\
\hline Apricots & 1,311 & $2,039+$ & $1,261+$ & $1,464 t$ & $744+$ & $931+$ \\
\hline Cherries & 522 & $787 t$ & $710+$ & $449+$ & $295+$ & $313+$ \\
\hline \multirow[t]{2}{*}{ Grapes } & 30,523 & 29,980 & 39,928 & 26,906 & 17,961 & 16,982 \\
\hline & \multicolumn{6}{|c|}{ Short tons, net declared dry weight } \\
\hline Dried: & & & & & & \\
\hline Total listed & 70,043 & 161,892 & 234,469 & 199,871 & 212,575 & 218,942 \\
\hline Deciduous tree & 26,877 & 98,507 & 157,847 & 138,886 & 154,328 & 139,642 \\
\hline Prunes & 19,017 & 59,523 & 107,016 & 96,313 & 106,836 & 103,240 \\
\hline Apples & 1,334 & 8,370 & 15,815 & 13,341 & 16,735 & 14,846 \\
\hline Apricots & 1,454 & 15,961 & 14,420 & 13,305 & 15,126 & 9,766 \\
\hline Fruit salad & 1,791 & 7,806 & 10,431 & 6,870 & $-\neq$ & - \\
\hline Pears & 393 & 2,466 & 4,358 & 3,489 & 3,244 & -- \\
\hline Peaches & 802 & 2,580 & 4,119 & 3,408 & 3,604 & 3,396 \\
\hline Other & 2,086 & 1,801 & 1,688 & 2,160 & 8,783 丮 & $8,394 \neq$ \\
\hline \multirow[t]{2}{*}{ Raisins } & 43,166 & 63,385 & 76,622 & 60,985 & 58,247 & 79,300 \\
\hline & \multicolumn{6}{|c|}{ Short tons, net declared canned vei ght } \\
\hline \multicolumn{7}{|l|}{ Canned: } \\
\hline Total listed & 6,551 & 157,493 & 165,958 & 139,106 & 121,415 & 117,767 \\
\hline Peaches & 1,982 & 43,205 & 48,326 & 36,666 & 37,299 & 41,052 \\
\hline Pears & 1,224 & 30,958 & 38,644 & 35,699 & 33,978 & 33,115 \\
\hline Fruit salad & 1,656 & 23,194 & 21,574 & 18,564 & 16,126 & $--\neq$ \\
\hline Grapefruit & 240 & 26,851 & 22,294 & 17,266 & - 丮 & -- \\
\hline Apricots & 305 & 16,572 & 16,538 & 12,104 & 11,924 & 15,206 \\
\hline Pineapple & 792 & 10,250 & 11,082 & 11,403 & 14,029 & 19,998 \\
\hline Apples & 352 & $6 ;, 463$ & 7,500 & 7,404 & 8,059 & 8,396 \\
\hline
\end{tabular}

* Some preliminary data, 1934 to date, had to be used in taoles 31 and 32 ; hence, preliminary totals are also used in this table for comparability.

+ only Canadian imports from United States used for crop years 1924-1939.

F Dashes indicate data not reported separately, but included in "other" dried or "other" canned fruits; "other" camned fruit tonnage excluded from this table: 1924$1928,14,530$; 1929-1933, 12,582; and 1934-1938, 6,289.

Source of data:

Compiled by S. W. Shear, Giannini Foundation, from official customs data,

largely from reports of the U. S. Department of Agriculture. 


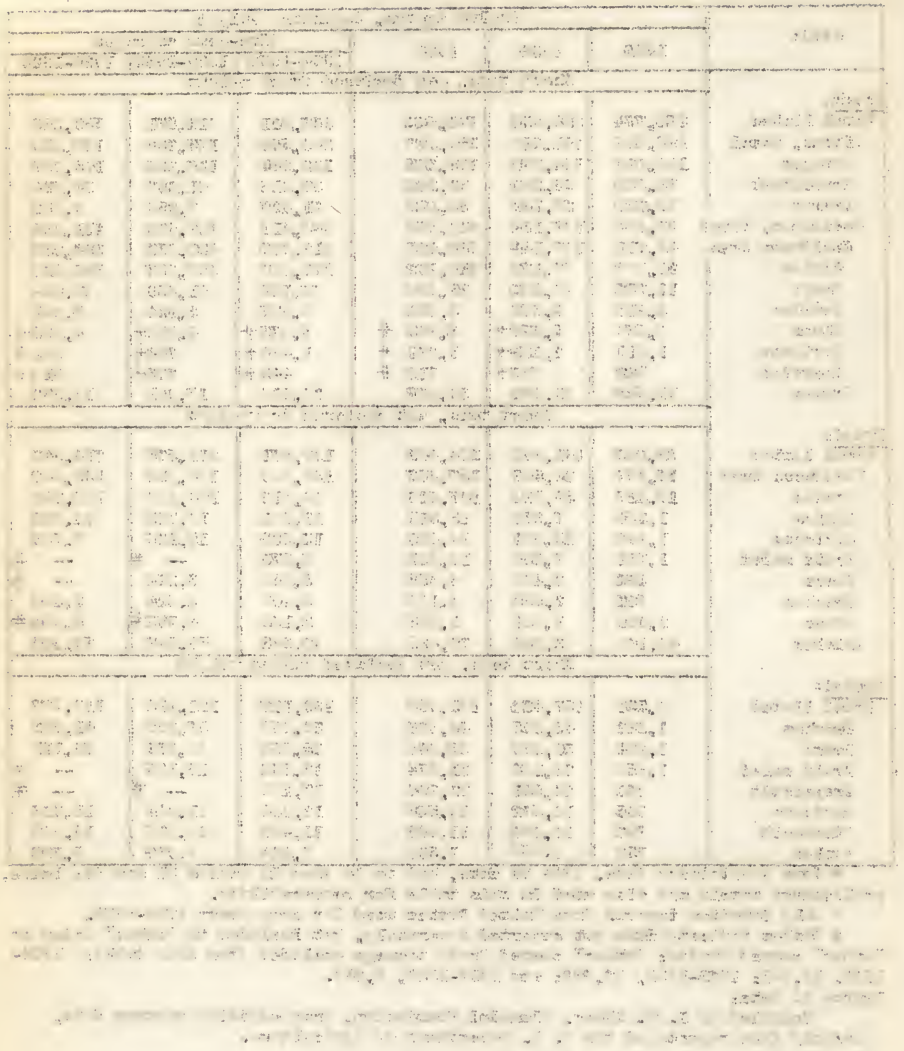


Table 31. United States Domestic Exports* of Fruits to Europe

\begin{tabular}{|c|c|c|c|c|c|}
\hline \multirow[b]{2}{*}{ Fruit } & \multirow{2}{*}{$\begin{array}{l}\text { Wine months } \\
\text { July-liaroh } \\
1940-41\end{array}$} & \multicolumn{4}{|c|}{ Twelve months, beginning July 1} \\
\hline & & 1939 & 1938 & 1937 & $\begin{array}{c}\text { Average } \\
1934-1938 \\
\end{array}$ \\
\hline \multirow{2}{*}{\multicolumn{6}{|c|}{ Short tons, net declared fresh weight }} \\
\hline & & & & & \\
\hline$\overline{\text { Total listed }}$ & 1,041 & 90,024 & 502,587 & 395,590 & 363,779 \\
\hline Citrus, total & 493 & 12,639 & 156,194 & 112,463 & 99,414 \\
\hline Oranges & 263 & 6,125 & 125,720 & $101,535+$ & $78,085+$ \\
\hline Grapefruit & 41 & 2,600 & 16,680 & $6,520+$ & 13,890 \\
\hline Lemons & 189 & $3,914+$ & 13,794 & $4,408+$ & 7,439 \\
\hline Deoiduous, total & 548 & 77,385 & 346,393 & 283,127 & 264,365 \\
\hline Deciduous tree & 440 & 73,213 & 328,327 & 270,823 & 256,068 \\
\hline Apples & 136 & 46,493 & 255,766 & 220,478 & 205,802 \\
\hline Pears & 304 & 26,700 & 71,509 & 50,261 & 50,206 \\
\hline Peaches & $0+$ & $20+$ & 52 & 84 & 60 \\
\hline Plums & $T$ & + & † & & $T$ \\
\hline Apricots & $T$ & $\mp$ & $\neq$ & & \\
\hline Cherries & $\neq$ & + & $\neq$ & $\neq$ & \\
\hline \multirow[t]{2}{*}{ Grapes } & & 4,172 & 18,066 & 12,304 & 8,297 \\
\hline & \multirow{2}{*}{\multicolumn{5}{|c|}{ Short tons, net declared dry weight }} \\
\hline Dried: & & & & & \\
\hline Total listed & 21,852 & 129,140 & 204,844 & 186,236 & 167,061 \\
\hline Deciduous tree & 4,881 & 77,496 & 138,501 & 126,203 & 117,838 \\
\hline Prunes & 2,141 & 43,548 & 91,597 & 88,296 & 79,459 \\
\hline Apples & 566 & 7,653 & 15,171 & 11,624 & 12,769 \\
\hline Apricots & 79 & 14,099 & 13,300 & 14,527 & 12,076 \\
\hline Fruit salad & 1,068 & 7,467 & 10,126 & 6,156 & 6,589 \\
\hline Pears & 123 & 2,210 & 4,107 & 2,468 & 3,200 \\
\hline Peaches & 74 & 1,630 & 3,238 & 1,971 & 2,404 \\
\hline Other & 830 & 789 & 962 & 1,161 & 1,341 \\
\hline \multirow[t]{2}{*}{ Raisins } & 16,971 & 51,644 & 66,343 & 60,033 & 49,223 \\
\hline & \multicolumn{5}{|c|}{ Short tons, net deolared canned weight } \\
\hline Canned: & & & & & \\
\hline Total listed & 515 & 149,453 & 158,921 & 115,926 & 132,182 \\
\hline Peaches & 98 & 40,943 & 46,237 & 26,260 & 34,701 \\
\hline Pears & 90 & 29,432 & 37,250 & 28,199 & 34,424 \\
\hline Fruit salad & 202 & 21,324 & 20,100 & 14,915 & 17,368 \\
\hline Grapefruit & 82 & 26,590 & 21,972 & 17,050 & 16,954 \\
\hline Apricots & 8 & 16,028 & 16,014 & 12,839 & 11,593 \\
\hline Pin eapple & 31 & 8,972 & 10,092 & 10,946 & 9,954 \\
\hline Apples & 4 & 6,164 & 7,256 & 5,717 & 7,188 \\
\hline
\end{tabular}

* Some preliminary data had to be used in preparing this table.

+prinor estimates involved.

F Minor fruits omitted as data unavailable every year; most exports of fresh plums, aprioots, and cherries are to countries other than Furope.

Source of data:

Compiled by S. W. Shear, Giannini Foundation, from offioial

preliminary custons data. 
$\therefore \quad \because \pi \cdot-2$,

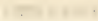

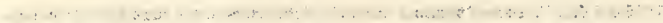

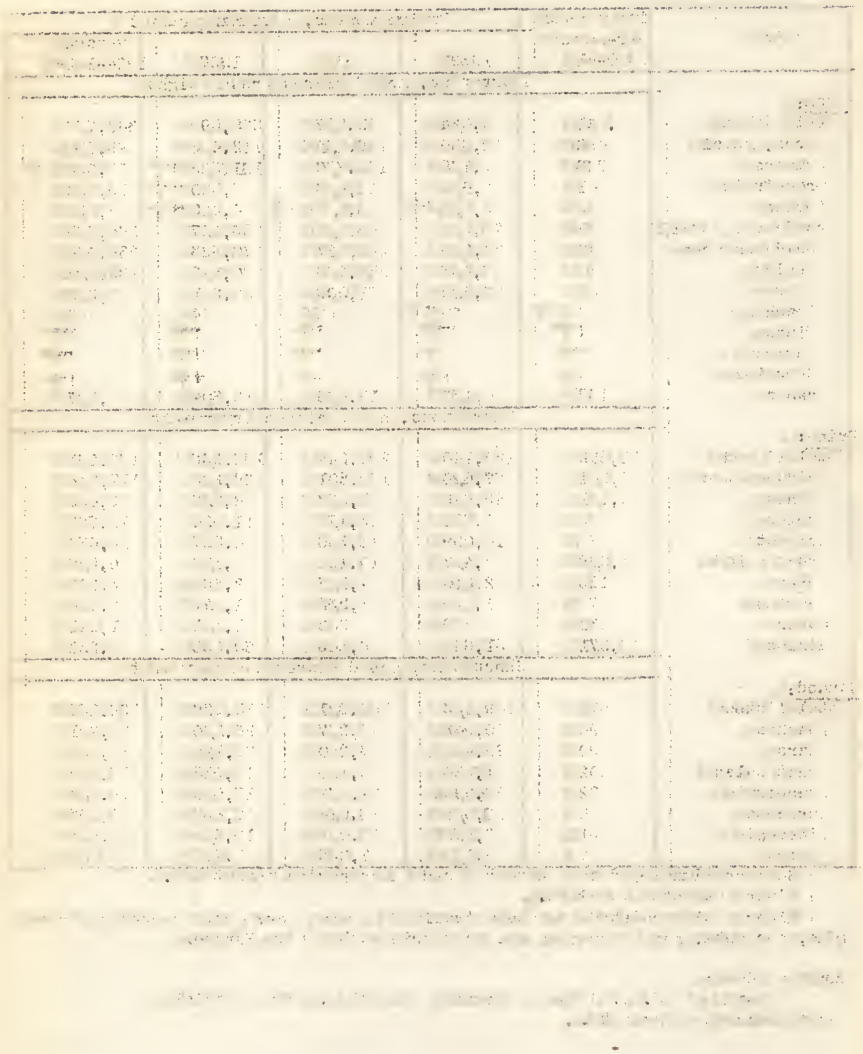


Table 32. United States Domestic Exports* of Fruits to Countries Other Than Furope

\begin{tabular}{|c|c|c|c|c|c|}
\hline \multirow[b]{2}{*}{ Fruit } & \multirow{2}{*}{$\begin{array}{l}\text { Nine months } \\
\text { July-Iarch, } \\
1940-41\end{array}$} & \multicolumn{4}{|c|}{ Twelve months, beginning July 1} \\
\hline & & 1939 & 1938 & 1937 & $\begin{array}{c}\text { A verage } \\
1934-1938\end{array}$ \\
\hline & \multicolumn{5}{|c|}{ Short tons, net declared fresh weight } \\
\hline \multicolumn{6}{|c|}{ 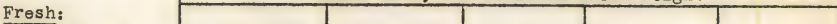 } \\
\hline $\begin{array}{l}\text { Total listed } \\
\text { Citrus total }\end{array}$ & $\begin{array}{l}208,553 \\
141,025\end{array}$ & $\begin{array}{l}268,410 \\
178,671\end{array}$ & 274,346 & 240,249 & 223,622 \\
\hline oranges & 104,586 & 128,765 & 139,400 & $147,405+$ & $144,176+$ \\
\hline Grapefruit & 23,137 & 30,640 & 35,560 & $100,080 \mathrm{~T}$ & 104, $860 \mathrm{~T}$ \\
\hline Lemons & 13,302 & $19,266+$ & $19,228+$ & $12,920 \mathrm{~F}$ & 25,726 \\
\hline Deciduous total & 67,528 & 89,739 & 79,943 & 92,844 & 13,590 \\
\hline Deciduous tree & 37,306 & 63,931 & 58,081 & 70,134 & 60,837 \\
\hline Apples & 17,619 & 30,679 & 32,936 & 42,502 & 34,605 \\
\hline Pears & 11,194 & 19,830 & 13,977 & 17,113 & 15, \\
\hline Peaches & $3,832+$ & $4,740+$ & 5,332 & 3,511 & 4,868 \\
\hline Plums & 3,420 & $5,856+$ & $3,865+$ & $3,664+$ & 3,475 \\
\hline Apricots & $1,118 \%$ & $2,039+$ & $1,251 \div$ & $2,811+$ & $1,464+$ \\
\hline Cherries & 123 & $787 \mp$ & $710 \neq$ & $533+$ & $449 \neq$ \\
\hline Grapes & 30,222 & 25,808 & 21,862 & 22,710 & 18,609 \\
\hline & \multicolumn{5}{|c|}{ Short tons, net declared dry weight } \\
\hline \multicolumn{6}{|l|}{ Dried: } \\
\hline Total listed & 22,260 & 32,752 & 29,625 & 35,163 & 32,810 \\
\hline Deciduous tree & 15,556 & 21,011 & 19,346 & 24,511 & 21,048 \\
\hline Prunes & 12,248 & 15,875 & 15,419 & 19,100 & 16,854 \\
\hline Apples & 310 & 717 & 644 & 594 & 572 \\
\hline Apricots & 1,026 & 1,862 & 1,120 & 1,898 & 1,229 \\
\hline Fruit salad & 135 & 339 & 305 & 333 & 281 \\
\hline Pears & 183 & 256 & 251 & 321 & 289 \\
\hline Peaches & 593 & 950 & 881 & 1,203 & 1,004 \\
\hline other & 1,061 & 1,012 & 726 & 1,062 & 819 \\
\hline Raisins & 6,704 & 11,741 & 10,279 & 10,652 & 11,762 \\
\hline & \multicolumn{5}{|c|}{ Short tons, net declared canned weight } \\
\hline Canned: & & & & & \\
\hline Total $l$ isted & 4,747 & 8,040 & 7,037 & 7,528 & 24 \\
\hline Peaches & 1,458 & 2,262 & 2,089 & 1,934 & $\begin{array}{l}1,965 \\
1,275\end{array}$ \\
\hline Pears & 865 & 1,526 & $\begin{array}{l}1,394 \\
1,474\end{array}$ & $\begin{array}{l}1,363 \\
1,386\end{array}$ & $\begin{array}{l}275 \\
196\end{array}$ \\
\hline $\begin{array}{l}\text { Fruit salad } \\
\text { Grapefruit }\end{array}$ & $\begin{array}{r}1,157 \\
129\end{array}$ & $\begin{array}{l}870 \\
261\end{array}$ & $\begin{array}{r}1,474 \\
322\end{array}$ & $\begin{array}{l}386 \\
328\end{array}$ & $\begin{array}{l}196 \\
312\end{array}$ \\
\hline Apricots & 228 & 544 & 524 & 626 & 511 \\
\hline Pineapple & 657 & 1,278 & 990 & 1,646 & 1,449 \\
\hline Apples & 253 & 299 & 244 & 245 & 216 \\
\hline
\end{tabular}

* Some preliminary data had to be used in preparing this table.

+Minor estimates involved.

f anly Canadian imports from United States used for crop years 1934-1939. Europe.

\$ Total U. S. assuming no exports of fresh plums, apricots, and cherries to

\section{Source of data:}

Compiled by S. W. Shear, Giannini Foundation, from official preliminary customs data. 


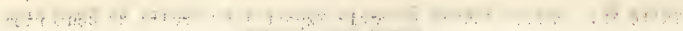

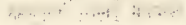

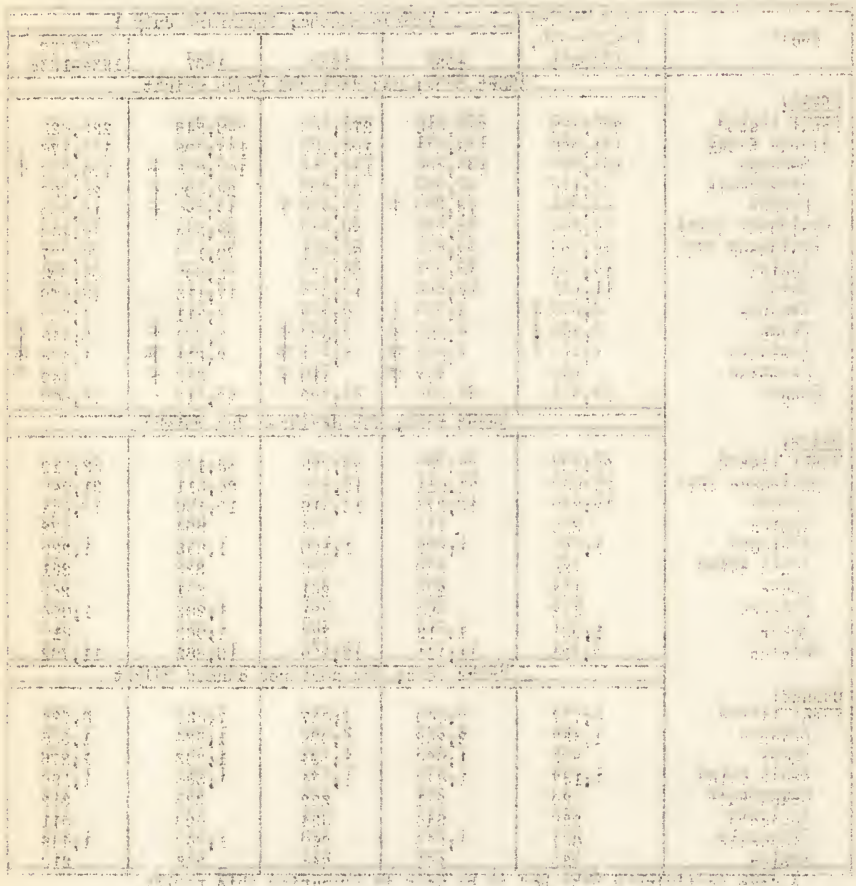

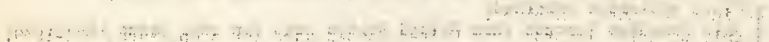

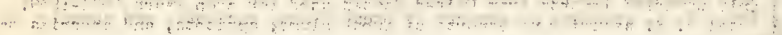

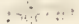

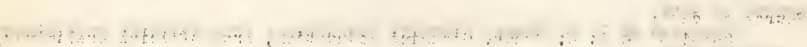

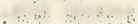


Table 33. United States Exports* of Fresh Fruits to Chief Countries , Average Years Beginning July 1, 1934-1938

\begin{tabular}{|c|c|c|c|c|c|c|c|c|c|c|}
\hline \multirow[b]{2}{*}{ Fruit } & \multirow[b]{2}{*}{$\begin{array}{l}\text { Total } \\
\text { exports }\end{array}$} & \multicolumn{7}{|c|}{ Europe } & \multicolumn{2}{|c|}{ Except Europe } \\
\hline & & $\begin{array}{l}\text { United } \\
\text { Kingdom }\end{array}$ & France & $\begin{array}{l}\text { Nether- } \\
\text { lands }\end{array}$ & Belgium & $\begin{array}{l}\text { Scandi- } \\
\text { navia }\end{array}$ & Germany $\oint$ & $\begin{array}{l}\text { Other } \\
\text { Europe }\end{array}$ & Canada & $\begin{array}{c}\text { Other } \\
\text { countries }\end{array}$ \\
\hline & \multicolumn{10}{|c|}{ Short tons, net declared fresh weight } \\
\hline Total, listed & 591,434 & 204,678 & 50,176 & 33,059 & 24,988 & 26,567 & 18,608 & 7,741 & 168,373 & 57,244 \\
\hline Citrus, totel & 243,590 & 61,358 & 11,683 & 8,161 & 5,030 & 9,563 & 1,714 & 1,905 & 129,703 & 14,473 \\
\hline Oranges & 182,945 & 43,694 & 10,465 & 7,105 & 4,470 & 8,032 & 1,541 & 1,7789 & 93,835 & 11,025 에 \\
\hline Grapefruit & 39,616 & 11,440 & 880 & 600 & 279 & 427 & 160 & 104 & 24,088 & 1,638 \\
\hline Lemons & 21,029 & 6,224 & 338 & 456 & 281 & 104 & 13 & 23 & 11,780 & 1,810 \\
\hline Deciduous, listed & 347,844 & 143,320 & 38,493 & 24,898 & 19,958 & 17,004 & 16,894 & 5,836 & 38,670 & 42,771 \\
\hline Apples & 240,407 & 102,256 & 29,702 & 21,306 & 19,181 & 12,590 & 15,972 & 4,795 & 6,212 & 28,393 \\
\hline Pears & 66,182 & 31,935 & 8,765 & 3,540 & 768 & 3,592 & 876 & 730 & 9,104 & 6,872 \\
\hline Grapes & 26,906 & 7,116 & 14 & 35 & -11 & 811 & 46 & 275 & 11,970 & 6,639 \\
\hline Peaches & 4,928 & 55 & -- & 0 & 0 & 5 & - & 0 & 4,650 & 218 \\
\hline Plums and prunes & 6,972 & 1,953 & 12 & 14 & 5 & 4 & - & 34 & 4,401 & 549 \\
\hline Apricots & 1,893 & 4 & 0 & 3 & 0 & 2 & 0 & 1 & 1,844 & 39 \\
\hline Cherries & 556 & 1 & - & 0 & 4 & 0 & 0 & 1 & 489 & 61 \\
\hline
\end{tabular}

* Some preliminary data had to be used in preparing this table.

+ United States exports by chief countries of destination are given for most fresh fruits for individual years beginning July 1, 1931-1938 in U. S. Dept. Agr., Agricultural Statistics, 1940, pp. 504 and 505.

\# Data are for years beginning July 1, except data on peaches are for calendar years 1934-1938, plums and fresh prunes and cherries are for calendar years 1936-1938, and apricots are for calendar years 1937 and 1938.

$\$$ Data for Germany include Austria beginning May 6, 1938; Sudeten area as far as ascertainable, beginning November 10, 1938; Czecko-Slovak provinces occupied by Germany beginning March 18 or 19, 1939; and kemel, beginning March 25, 1939.

9 Partly estimated.

" Dashes indicate less than 0.5 of a ton.

Source of data: Compiled by S. W. Shear, Giannini Foundation, from official preliminary customs data. Oranges converted at 70 pounds per box; grapefruit. at 80 pounds per box; lemons at 76 pounds per box; and apples converted at 1 box $=1$ basket $=1$ bushel $=48$ pounds, and 1 barrel $=3$ bushels. 
w

r

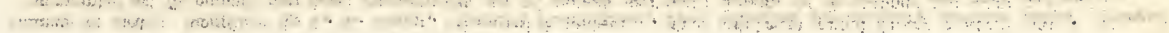

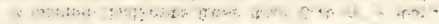

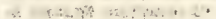

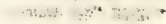

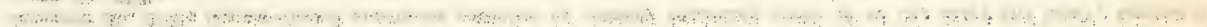

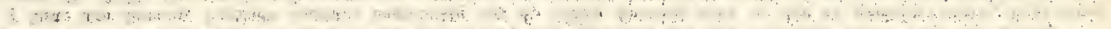

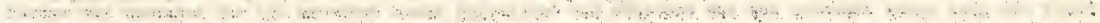

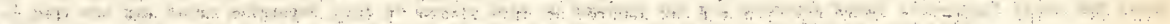

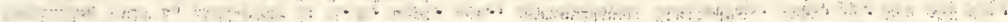

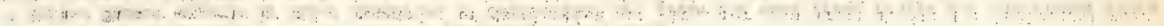

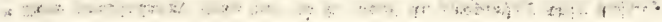

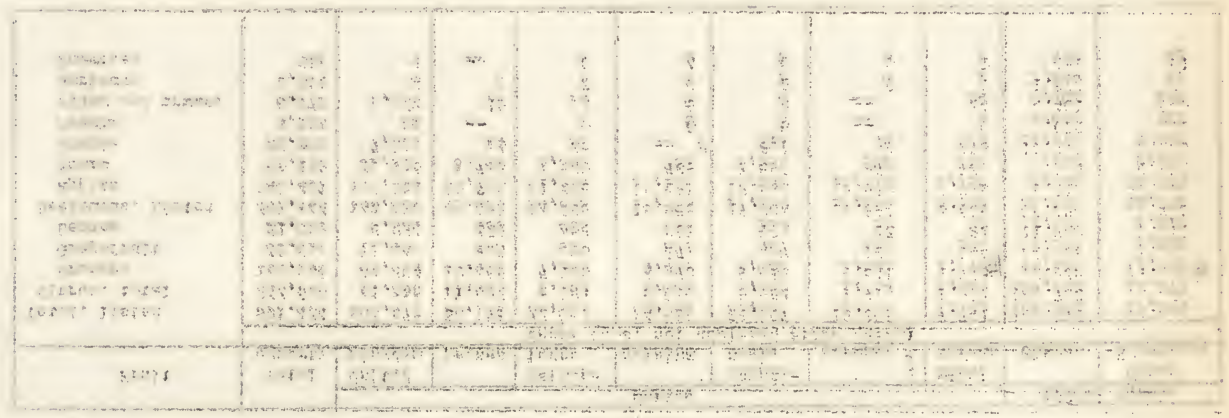

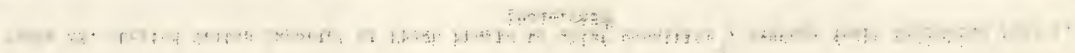

$\cdots \quad \therefore \quad \cdots, \because$ 
Table 34. United States Domestic Exports* of Dried and Canned Fruits to Chief Countries Average Years Beginning July 1, 1934-1938

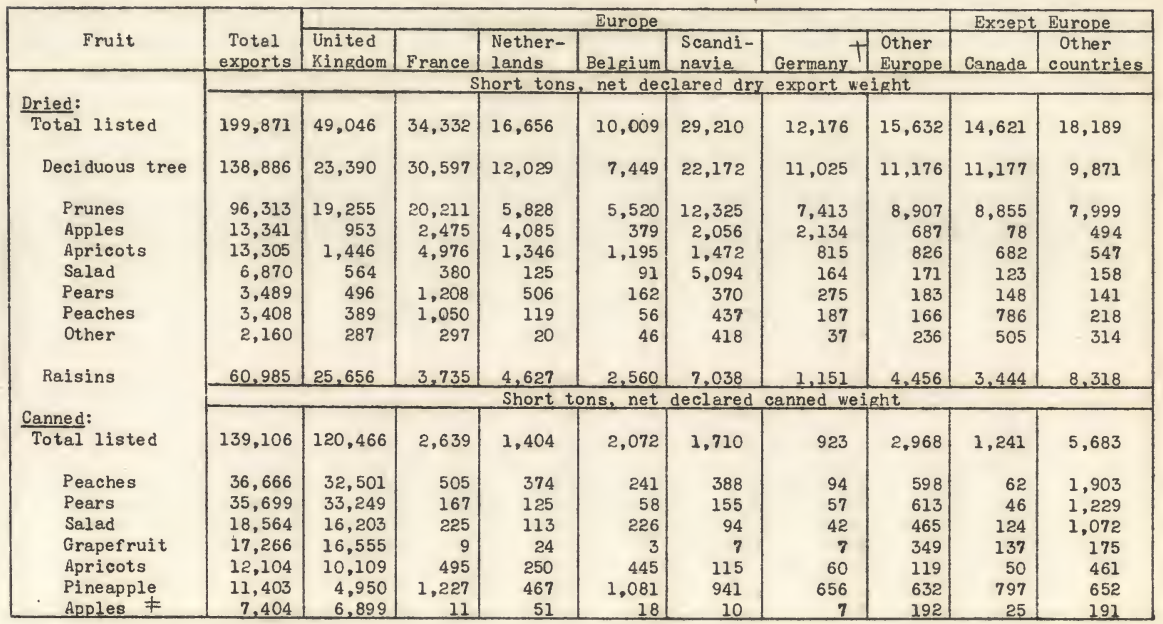

* Some preliminary data had to be used in preparing this table.

+ Data for Germany include Austria beginning May 6, 1938; Sudeten area as far as ascertainable, beginning Nov. 10, 1938; Czecko-Slovak provinces occupied by Germany beginning March 18 or 19, 1939; and Meme1, beginning March 25, 1939. * Canned apples include applesauce.

Source of data: Compiled by S. W. Shear, Giannini Foundation, from official preliminary customs data. United States exports by chief countries of destination are given for most dried and canned fruits for individual years beginning July 1, 1931-1938, in U. S. Dept. Agr., Agricultural Statistics, 1940, pp. 505-508. 


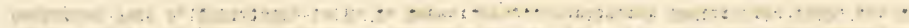

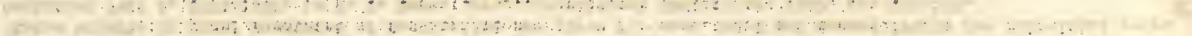

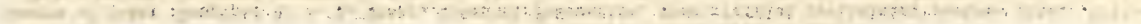

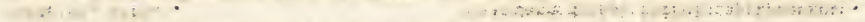

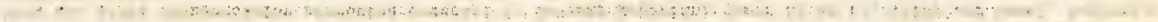

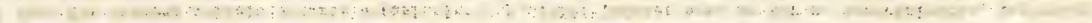

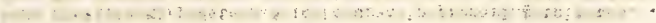

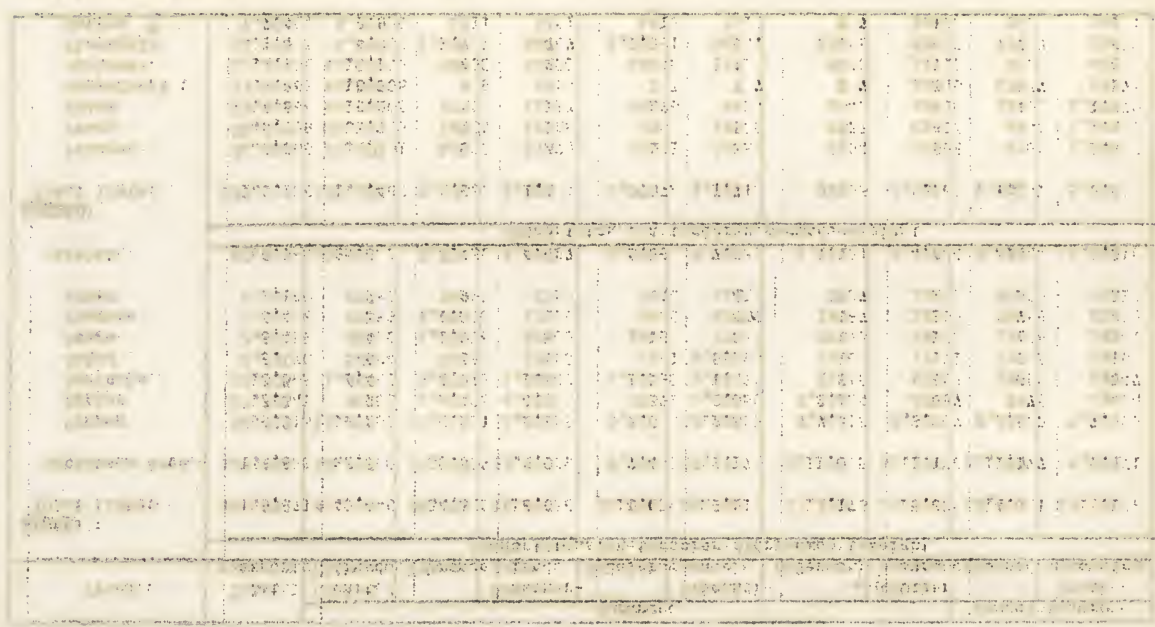

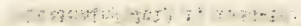

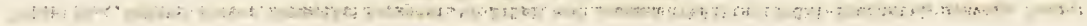


University of California, College of Agriculture Agricultural Experiment Station, Berkeley, January, 1942

DECIDUOUS FRUIT STATISTICS

APPLES

Table 1

California Apples: Bearing Acreage, Production, Condition, Yield, and Farm Value, 1919-1941

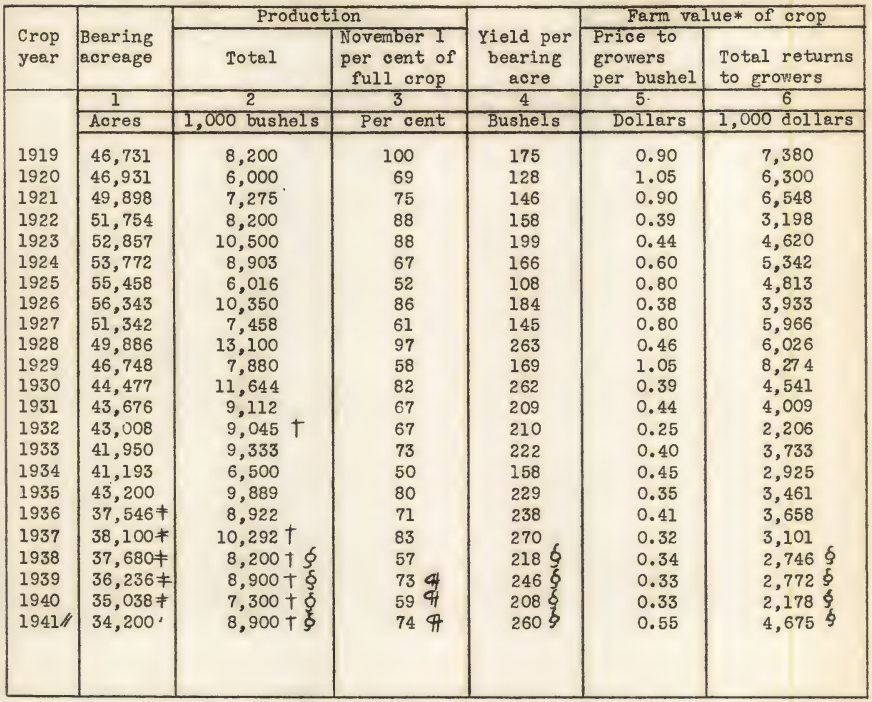

* Growers' returns for naked fruit delivered at growers' first delivery point.

+ Includes unharvested in bushels: $1932,220,000 ; 1937,600,000 ; 1938$, 124,000 ; and unofficial estimates by S. V. Shear in $1939,500,000 ; 1940,700,000$; and $1941,400,000$.

₹ Nonbearing acres: $1936,2,688 ; 1937,2,693 ; 1938,2,495 ; 1939,2,294$, and $1940,2,171$.

$\$$ Unofficial estimates by S. W. Shear.

q Data on per cent of full crop for 1939-1941 are for commercial canties anly. Preliminary estimate.

Source of data: Compiled by s. W. Shear, Giannini Foundation, from latest estinates of the California Crop Reporting Service, except as indicated by footnote $\delta$ for 1938-1941 and cols. 4 and 6 calculated. 

APPLES

Table 2. California Apples: Production and Utilization, 1916-1941

\begin{tabular}{|c|c|c|c|c|c|}
\hline \multirow[b]{2}{*}{ Crop year } & \multicolumn{3}{|c|}{ Harvested production } & \multirow[b]{2}{*}{$\begin{array}{l}\text { Used for } \\
\text { drying } T\end{array}$} & \multirow[b]{2}{*}{$\begin{array}{l}\text { Other then } \\
\text { commercial } \\
\text { and dried } \neq\end{array}$} \\
\hline & Total & $\begin{array}{l}\text { Commercial,* } \\
\text { sold for } \\
\text { fresh use }\end{array}$ & $\begin{array}{l}\text { Other } \\
\text { uses }\end{array}$ & & \\
\hline & 1 & 2 & 3 & 4 & 5 \\
\hline & & Short tons, & esh equiva & nt weight & \\
\hline 1916 & 166,320 & 84,528 & 81,792 & 33,750 & 48,042 \\
\hline 1917 & 163,296 & 84,528 & 78,768 & 63,750 & 15,018 \\
\hline 1918 & 157,440 & 81,144 & 76,296 & 47,250 & 29,046 \\
\hline 1919 & 196,800 & 82,800 & 114,000 & 93,750 & 20,250 \\
\hline 1920 & 144,000 & 88,560 & 55,440 & 41,250 & 14,190 \\
\hline 1921 & 174,600 & 102,000 & 72,600 & 52,500 & 20,100 \\
\hline 1922 & 196,800 & 100,728 & 96,072 & 78,750 & 17,322 \\
\hline 1923 & 252,000 & 151,200 & 100,800 & 71,250 & 29,550 \\
\hline 1924 & 213,672 & 107,280 & 106,392 & 75,000 & 31,392 \\
\hline 1925 & 144,384 & 78,984 & 65,400 & 43,500 & 21,900 \\
\hline 1926 & 248,400 & 147,456 & 100,944 & 78,750 & 22,194 \\
\hline 1927 & 178,992 & 111,744 & 67,248 & 47,250 & 19,998 \\
\hline 1928 & 314,400 & 164,664 & 149,736 & 122,250 & 27,486 \\
\hline 1929 & 189,120 & 105,912 & 83,208 & 67,500 & 15,708 \\
\hline 1930 & 279,456 & 156,528 & 122,928 & 80,250 & 42,678 \\
\hline 1931 & 218,688 & 111,528 & 107,160 & 79,500 & 27,660 \\
\hline 1932 & $211,800 \delta$ & 120,408 & 91,392 & 73,500 & 17,892 \\
\hline 1933 & 223,992 & 91,896 & 132,096 & 99,750 & 32,346 \\
\hline 1934 & 156,000 & 82,872 & 73,128 & 66,000 & 7,128 \\
\hline 1935 & 237,336 & 123,888 & 113,448 & 93,750 & 19,698 \\
\hline 1936 & 214,128 & 117,288 & 96,840 & 87,750 & 9,090 \\
\hline 1937 & $232,608 \oint$ & 132,696 & 99,912 & 90,000 & 9,912 \\
\hline 19389 & $193,824 \zeta థ$ & 120,456 & 73,3689 & 56,250 & $17,118 \&$ \\
\hline 19394 & 201,600 б & 108,0004 & 93,6009 & 81,750 & $11,850 \AA$ \\
\hline $1940 q^{n}$ & 158,40069 & 96,000 क & $62,400 \%$ & 36,000 & 26,400 A \\
\hline 19419 & $204,000 \$ 9$ & $100,800 \propto$ & 103,2004 & 71,0009 & 32,2009 \\
\hline
\end{tabular}

* The old commercial production series given here includes apples sold for fresh consumption only and excludes dried, canned, or otherwise commercially processed, and consumed on farms where grown. See footnote to table 4 about new commercial production series.

+ Converted from dry tons to fresh equivalent by multiplying by $7 \frac{1}{2}$.

F Mostly used for cider, vinegar, and other by-products or wasted.

of Excludes unharvested tonnage: 1932, 5,280; 1937, 14,400; 1938, 2,976; and unofficial estimates by S. W. Shear in 1939, 12,000; 1940, 16,800; and $1941,9,600$.

A Preliminary unofficial estimates of S. W. Shear.

Sources of data: Compiled by S. \%. Shear, Giannini Foundation of Agricultural

Economics, University of Cel ifornia.

Cols. 1 and 2: Official estimates of United States and California Crop

Reporting Service. Converted to tons from bushels at 48 pounds per bushel.

Cols. 3 and 5: Calculated from other data in table.

Col. 4: 1916-1922 are trade estimates from California Fruit News. 1923-1940 based largely on packers' receipts as reported to Dried Fruit Association of California. Converted at a drying ratio of $7 \frac{1}{2}$ to 1 . 


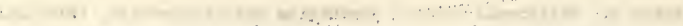

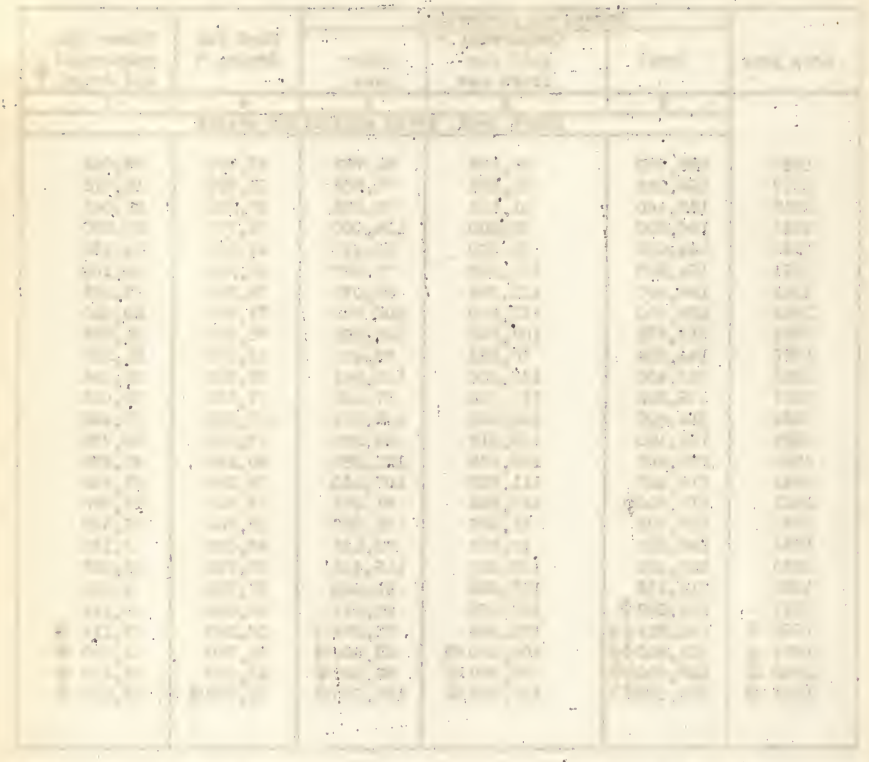

Th

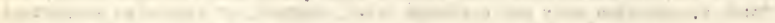

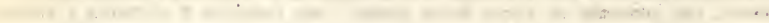

(20)

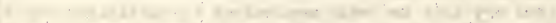

ant

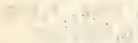

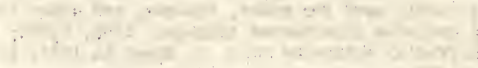
, in: F (2)

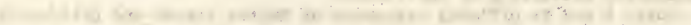

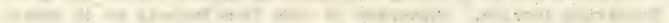
-

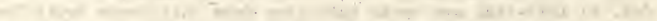

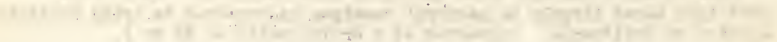


January 1942

\section{APPLES}

Tablo 3; Sonoma-Napa Gravenstein ipples: Production, Utilization, and Growers' Price of Fresh Shipments, 1923-1941

\begin{tabular}{|c|c|c|c|c|c|c|}
\hline \multirow{2}{*}{ Crop year } & \multirow{2}{*}{ Total } & \multirow{2}{*}{$\begin{array}{c}\text { Used for } \\
\text { drying }\end{array}$} & \multicolumn{3}{|c|}{ Fresh shipinents } & \multirow{2}{*}{$\begin{array}{l}\text { Price to } \\
\text { growers* per } \\
\text { packed box }\end{array}$} \\
\hline & & & Tote]. & Exported & Donestic & \\
\hline \multirow{5}{*}{$\begin{array}{l}\text { Averages: } \\
1923-1927 \\
1928-1932 \\
1933-1937\end{array}$} & 1 & 2 & $z$ & 4 & 5 & 6 \\
\hline & \multicolumn{5}{|c|}{ Thousands of boxes, fresh equivalent weight } & Dollars \\
\hline & & 494 & & & & \\
\hline & $\begin{array}{l}1,281 \\
2,103\end{array}$ & $\begin{array}{l}494 \\
798\end{array}$ & $\begin{array}{r}787 \\
1,305\end{array}$ & $\begin{array}{l}179 \\
608\end{array}$ & $\begin{array}{l}608 \\
697\end{array}$ & $\begin{array}{l}1.16 \\
0.65\end{array}$ \\
\hline & 2,268 & 1,280 & 988 & 324 & 664 & 0.47 \\
\hline \multicolumn{7}{|l|}{ Annual: } \\
\hline 1923 & 1,556 & 445 & 1,111 & 220 & 891 & 0.73 \\
\hline 1924 & 1,275 & 533 & 742 & 200 & 542 & 1.15 \\
\hline 1925 & 639 & 355 & 284 & 37 & 247 & 1.96 \\
\hline 1926 & 1,801 & 711 & 1,090 & 130 & 900 & 0.39 \\
\hline 1927 & 1,134 & 427 & 707 & 310 & 397 & 1.56 \\
\hline 1928 & 2,478 & 1,031 & 1,447 & 500 & 947 & 0.48 \\
\hline 1929 & 1,421 & 471 & 950 & 400 & 550 & 1.42 \\
\hline 1930 & 2,093 & 711 & 1,382 & 660 & 722 & 0.61 \\
\hline 1931 & 2,198 & 995 & 1,203 & 750 & 453 & 0.58 \\
\hline 1932 & 2,323 & 782 & 1,541 & 730 & 811 & 0.17 \\
\hline 1933 & 2,417 & 1,245 & 1,172 & 570 & 602 & 0.28 \\
\hline 1934 & 1,465 & 889 & 576 & 169 & 407 & 0.65 \\
\hline 1935 & 2,745 & 1,600 & 1,145 & 408 & 737 & 0.47 \\
\hline 1936 & 1,926 & 1,067 & 859 & 260 & 599 & 0.60 \\
\hline 1937 & 2,786 & 1,600 & 1,186 & 212 & 974 & 0.34 \\
\hline 1938 & 1,113 & 630 & 483 & 114 & 369 & 0.46 \\
\hline 1939 & $2,603+$ & 1,745 & 858 & 190 & 668 & 0.32 \\
\hline 1940 & $995+$ & 453 & 542 & 18 & 524 & 0.58 \\
\hline $1941 \neq$ & $1,453+$ & 907 & 546 & $\delta$ & $\xi$ & 0.60 \\
\hline
\end{tabular}

* Return to growers for fancy grade excluding cost of package and packing.

+ Excludes unharvested tonnage: 1939, 3,000;1940, 3,500; 1941, 5,000.

\# Preliminary estimates. \& Data not available.

Sources of data: Compiled by S.W. Shear, Giannini Foundation of Agricultural Economics, University of California.

Col. 1: Col. 3 plus Col. 2; excludes quantity used for cider and vinear.

Col. 2: Based larsely on dried estimates by F. D. orrill, Calif. Dried Fruit Laboratories converted at an 8 to 1 dryino ratio and 45 pounds per box. Col. 3: Data for 1923-1933 lartely from compilations by the Division of Shipping Point Inspection of California State Departient of Africulture. Recent years from reports of manacers of special marketin prosans.

Col. 4: Data 1923-1926 are roukh estimates by S. N. Shear; for 1927-1940 fairly reliable data compiled by best informed factors in the industry. Col. 5: Col. 3 minus Col. 4 .

Col. 6: Based upon data from cooperative and private shippers. 

Table 4. Apple Production, Total and Commercial, United States, Western States,* and Califormia, 1916-1941

\begin{tabular}{|c|c|c|c|c|c|c|}
\hline \multirow[b]{2}{*}{$\begin{array}{l}\text { Crop } \\
\text { year }\end{array}$} & \multicolumn{3}{|c|}{ Total production } & \multicolumn{3}{|c|}{ Commercial production $t$} \\
\hline & United States & $\begin{array}{l}\text { Western } \\
\text { states* }\end{array}$ & California & United States & $\begin{array}{l}\text { Western } \\
\text { states* }\end{array}$ & California \\
\hline & 1 & 2 & 3 & 4 & 5 & 6 \\
\hline & \multicolumn{6}{|c|}{ Thousands of bushels } \\
\hline 1916 & 193,905 & 33,186 & 6,930 & 80,241 & 23,799 & 3,522 \\
\hline 1917 & 166,749 & 39,960 & 6,804 & 67,023 & 25,590 & 3,522 \\
\hline 1918 & 169,625 & 32,330 & 6,560 & 74,229 & 21,309 & 3,381 \\
\hline 1919 & 140,632 & 50,060 & 8,200 & 81,047 & 36,091 & 3,450 \\
\hline 1920 & 206,688 & 36,431 & 6,000 & 103,102 & 28,950 & 3,690 \\
\hline 1921 & 95,638 & 54,872 & 7,275 & 66,100 & 42,337 & 4,250 \\
\hline 1922 & 189,425 & 52,327 & 8,200 & 100,642 & 37,385 & 4,197 \\
\hline 1923 & 180,915 & 62,022 & 10,500 & 109,922 & 49,345 & 6,300 \\
\hline 1924 & $160,457 \neq$ & $43,490+$ & 8,903 & 88,776 & 33,523 & 4,470 \\
\hline 1925 & 152,424 & 51,191 & 6,016 & 101,080 & 42,574 & 3,291 \\
\hline 1926 & $229,656 \neq$ & $63,060 \neq$ & 10,350 & 123,550 & 44,277 & 6,144 \\
\hline 1927 & 115,708 & 47,153 & 7,458 & 79,254 & 38,323 & 4,656 \\
\hline 1928 & 177,813 & 68,295 & 13,100 & 109,938 & 51,322 & 6,861 \\
\hline 1929 & 135,092 & 51,764 & 7,880 & 89,270 & 40,054 & 4,413 \\
\hline 1930 & 156,617 & 64,186 & 11,644 & 105,432 & 52,073 & 6,522 \\
\hline 1931 & $205,403 \neq$ & $53,364 \neq$ & 9,112 & 113,207 & 39,408 & 4,647 \\
\hline 1932 & $146,849 \neq$ & $54,813 \neq$ & $9,045 \neq$ & 90,023 & 38,029 & 5,017 \\
\hline 1933 & $148,657 \neq$ & $50,171 \neq$ & 9,333 & 81,925 & 30,897 & 3,829 \\
\hline 1934 & $125,719 \mp$ & $50,097 \neq$ & 6,500 & 79,870 & 36,386 & 3,453 \\
\hline 1935 & $177,916 \neq$ & $53,601 \neq$ & 9,889 & 103,749 & 35,059 & 5,162 \\
\hline 1936 & 117,506 & 47,753 & 8,922 & 75,539 & 34,419 & 4,887 \\
\hline 1937 & $210,783 \neq$ & $53,432 \neq$ & $10,292 \neq$ & 115,733 & 35,835 & 5,529 \\
\hline 1938 & $132,354 \neq$ & $50,803=$ & $8,200 \neq \varepsilon$ & 82,395 & 35,320 & 5,019 \\
\hline 1939 & $186,000 \neq \delta$ & $46,000 \neq$ & $8,900 \neq \delta$ & $106,000 \xi$ & $31,500 \%$ & 4,5005 \\
\hline 1940 & $140,000 \neq \delta$ & $46,000=$ & $7,300 \neq \varnothing$ & 75,000 है & $32,500 \oint$ & $4,000 \delta$ \\
\hline 1941 & $160,000 \neq \varepsilon$ & 47,000 毒 & $8,900 \neq \delta$ & $90,000 \oint$ & 32,5005 & 4,2008 \\
\hline
\end{tabular}

* Western states included are Washington, Oregon, California, Idaho, Colorado, Nontana, Wyoming, New Liexico, Arizona, Utah, and Nevada.

T The old commercial production series given here includes apples sold for fresh consumption only and excludes dried, canned, or otherwise commercially processed and consumed on farms where grown. For 1939, 1940, and 1941 the only apple production estimates released by the Crop Reporting Service are a new commercial series for which data by states were given, 1934-1940 in vi. S. Dept. Agr., Crops and Narkets, Vol. 17, No. 8, August, 1940, p. 166, and current data from regular releases. The new commercial production series covers only commercial apple-producing counties and includes all uses in these counties -- dried, camed, and otherwise processed, as well as fresh consumption. The new estimates of commercial production are smaller than the old estimates of total production but generally larger than the old estimates of commercial production.

F Includes unharvested production.

f Rough unofficial estimates by s. W. Shear.

Sources of data: Compiled by S. W. Shear, Giannini Foundation of Agricultural Economics, University of California, from United Stotes and California Crop Reports except as indicated by footnote $\oint$ for 1938-1941. 


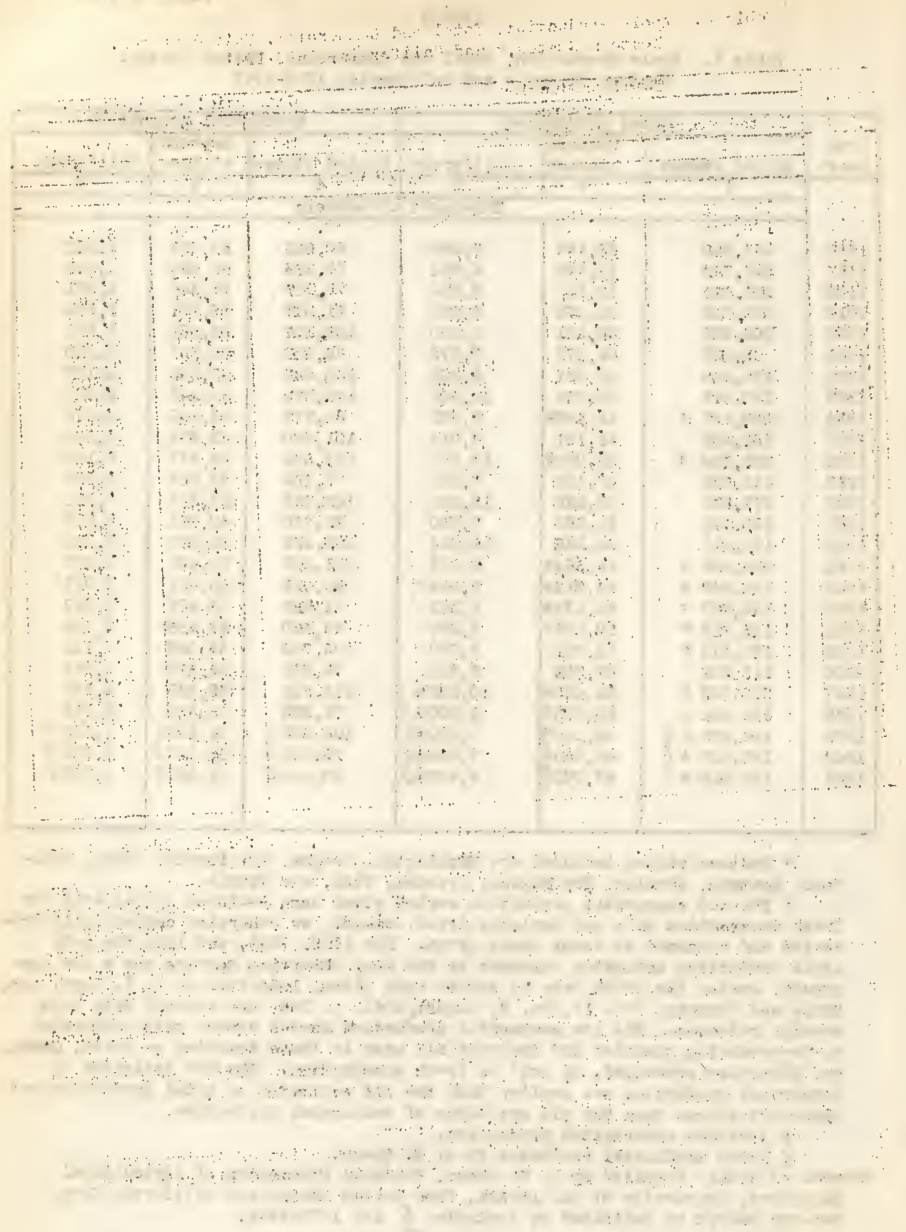


APPLES

January, 1942

Table 5. Fresh Apples: United States Domestic Exports by Countries of Destination, Years Beginning July 1, $1930-1940$

\begin{tabular}{|c|c|c|c|c|c|c|c|c|c|c|c|}
\hline $\begin{array}{l}\text { Country of } \\
\text { destination }\end{array}$ & $1930-31$ & $1931-32$ & $1932-33$ & $1933-34$ & $1934-35$ & $1935-36$ & $1936-37$ & $1937-38$ & $1938-39$ & $1939-40^{*}$ & $1940-41^{+}$ \\
\hline$\ldots$ & \multicolumn{11}{|c|}{ Short tons, net declared fresh weight } \\
\hline Total exports & 488,198 & 430,126 & 330,094 & 294,281 & 193,488 & 293,748 & 162,115 & 262,980 & 289,702 & 77,172 & $17,755+$ \\
\hline Europe, total & 436,505 & 401,635 & 308,002 & 270,763 & 169,188 & 258,401 & 124,176 & 220,478 & 256,766 & 46,493 & 136 \\
\hline $\begin{array}{l}\text { Uniter Kingdom } \\
\text { Other } \\
\text { France } \\
\text { Netherlands } \\
\text { Belgium } \\
\text { Norway } \\
\text { Sweden } \\
\text { Denmark } \\
\text { Germany } \neq \\
\text { Finland } \\
\text { Other }\end{array}$ & $\begin{array}{r}164,448 \\
272,057 \\
25,656 \\
82,080 \\
28,500 \\
3,113 \\
6,511 \\
9,490 \\
112,560 \\
1,740 \\
2,407\end{array}$ & $\begin{array}{r}219,813 \\
181,822 \\
48,312 \\
34,783 \\
15,646 \\
3,166 \\
11,251 \\
11,278 \\
52,994 \\
895 \\
3,497\end{array}$ & $\begin{array}{r}135,893 \\
172,109 \\
24,507 \\
43,771 \\
13,176 \\
2,174 \\
11,069 \\
3,257 \\
70,745 \\
1,819 \\
1,591\end{array}$ & $\begin{array}{r}82,519 \\
188,244 \\
30,881 \\
43,747 \\
20,827 \\
1,174 \\
5,326 \\
5,150 \\
77,175 \\
986 \\
2,978\end{array}$ & $\begin{array}{r}81,091 \\
88,097 \\
25,025 \\
15,734 \\
16,380 \\
2,381 \\
8,741 \\
1,150 \\
14,626 \\
1,358 \\
2,702\end{array}$ & $\begin{array}{r}146,868 \\
111,533 \\
33,722 \\
19,426 \\
25,920 \\
1,231 \\
9,372 \\
264 \\
18,960 \\
1,411 \\
1,227\end{array}$ & $\begin{array}{r}56,023 \\
68,153 \\
18,007 \\
13,519 \\
10,325 \\
1,639 \\
6,329 \\
269 \\
15,487 \\
2,194 \\
384\end{array}$ & $\begin{array}{r}103,090 \\
117,388 \\
37,344 \\
22,111 \\
22,503 \\
1,867 \\
8,678 \\
158 \\
18,907 \\
4,058 \\
1,762\end{array}$ & $\begin{array}{r}124,207 \\
132,559 \\
34,411 \\
35,741 \\
20,779 \\
4,315 \\
14,071 \\
2,484 \\
11,878 \\
5,011 \\
3,869\end{array}$ & $\begin{array}{r}34,229 \\
12,264 \\
19 \\
595 \\
2,016 \\
1,131 \\
6,492 \\
271 \\
\$ \\
86 \\
1,654\end{array}$ & $\begin{array}{r}30 \\
106 \\
0 \\
0 \\
0 \\
0 \\
4 \\
\$ \\
0 \\
91 \\
11\end{array}$ \\
\hline Except Europe & 93 & 28 & 992 & 23,518 & 24,300 & 35,347 & 37,939 & 42,502 & 32,936 & 30,679 & 17,619 \\
\hline $\begin{array}{l}\text { Canada } \\
\text { Other } \\
\text { Philippines } \\
\text { Brazil } \\
\text { Argentina } \\
\text { Cuba } \\
\text { Palestine } \\
\text { Other } \\
\end{array}$ & $\begin{array}{r}14,287 \\
37,406 \\
2,688 \\
4,087 \\
17,465 \\
1,603 \\
50 \\
11,513 \\
\end{array}$ & $\begin{array}{r}7,829 \\
20,662 \\
2,508 \\
3,041 \\
4,567 \\
1,157 \\
115 \\
9,274 \\
\end{array}$ & $\begin{array}{r}3,706 \\
18,386 \\
2,484 \\
3,067 \\
3,924 \\
1,027 \\
127 \\
7,757 \\
\end{array}$ & $\begin{array}{r}1,754 \\
21,764 \\
1,860 \\
2,182 \\
5,292 \\
905 \\
1,186 \\
10,339 \\
\end{array}$ & $\begin{array}{r}2,371 \\
21,929 \\
2,405 \\
2,817 \\
2,544 \\
1,481 \\
2,095 \\
10,587 \\
\end{array}$ & $\begin{array}{r}5,664 \\
29,683 \\
2,491 \\
3,773 \\
3,734 \\
1,994 \\
3,475 \\
14,216 \\
\end{array}$ & $\begin{array}{r}12,240 \\
25,699 \\
1,817 \\
2,937 \\
2,273 \\
1,858 \\
3,547 \\
13,267 \\
\end{array}$ & $\begin{array}{r}7,529 \\
34,973 \\
3,070 \\
3,353 \\
2,719 \\
2,796 \\
4,920 \\
18,115 \\
\end{array}$ & $\begin{array}{r}3,254 \\
29,682 \\
3,314 \\
3,046 \\
1,301 \\
2,830 \\
4,594 \\
14,597 \\
\end{array}$ & $\begin{array}{r}2,988 \\
27,691 \\
3,878 \\
3,521 \\
2,230 \\
3,314 \\
2,683 \\
12,065 \\
\end{array}$ & $\begin{array}{r}1,165 \\
16,454 \\
3,096 \\
1,735 \\
646 \\
3,001 \\
0 \\
7,976 \\
\end{array}$ \\
\hline
\end{tabular}

* Preliminary.

† Nine months only, July 1940-March 1941 as data by countries of destination not available for later months. Total exports for July-June were 20,808 tons.

\# Data for Germany include Austria beginning May 6, 1938, and parts of Czechoslovakia and Memel acquired since then.

$\dot{g}$ Less than 50 bushels.

Source of data: Compiled by S. Whear, Giannini Foundation of Agricultural Economics, from official daia of the U. S. Bureau of Foreign and Domestic Commerce, as compiled largely by the U. S. Dept. Agr., Office of Foreign Agricultural Relations. Converted to tons at 1 box $=1$ bushel, 1 barrel $=3$ bushels, and 1 bushel $=48$ pounds. 


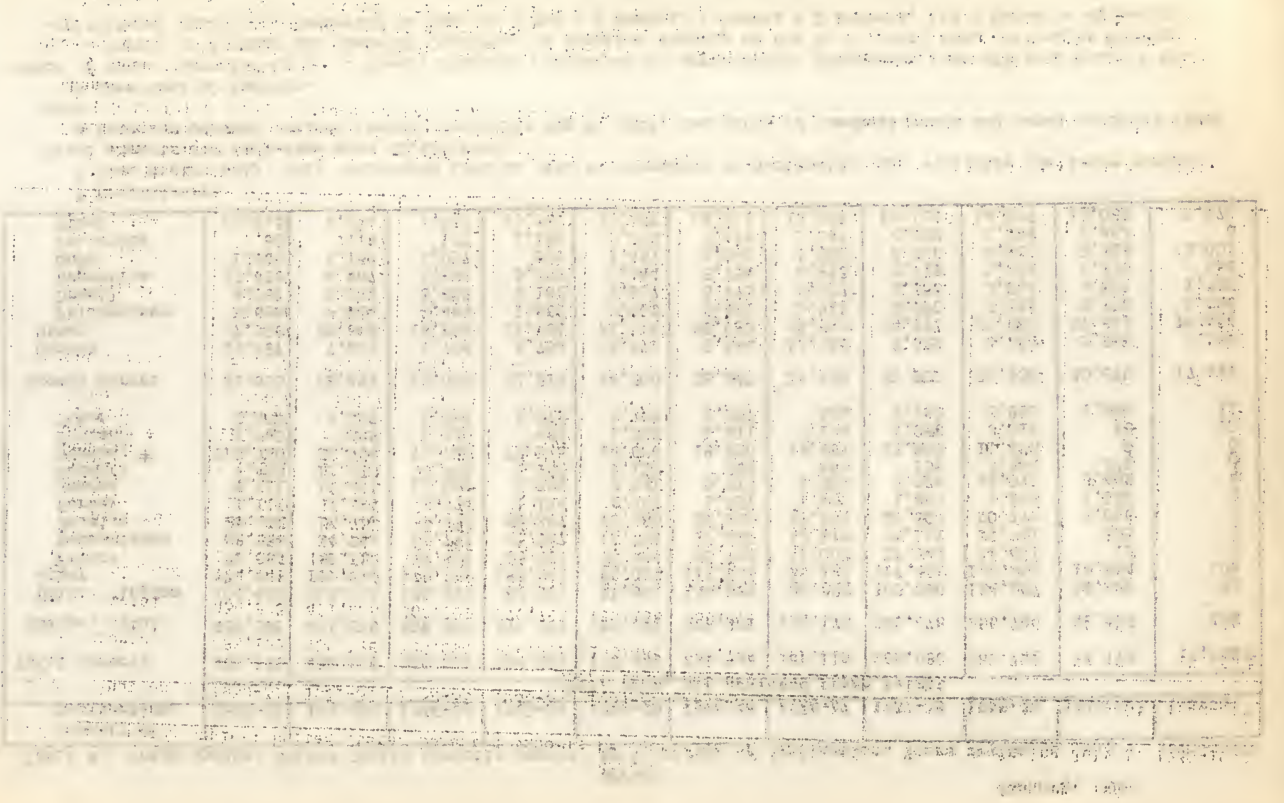


January, 1942

APPLES

Table 6. Dried Apples: United States Production and Exports and California Packers' Price, 1919-1941

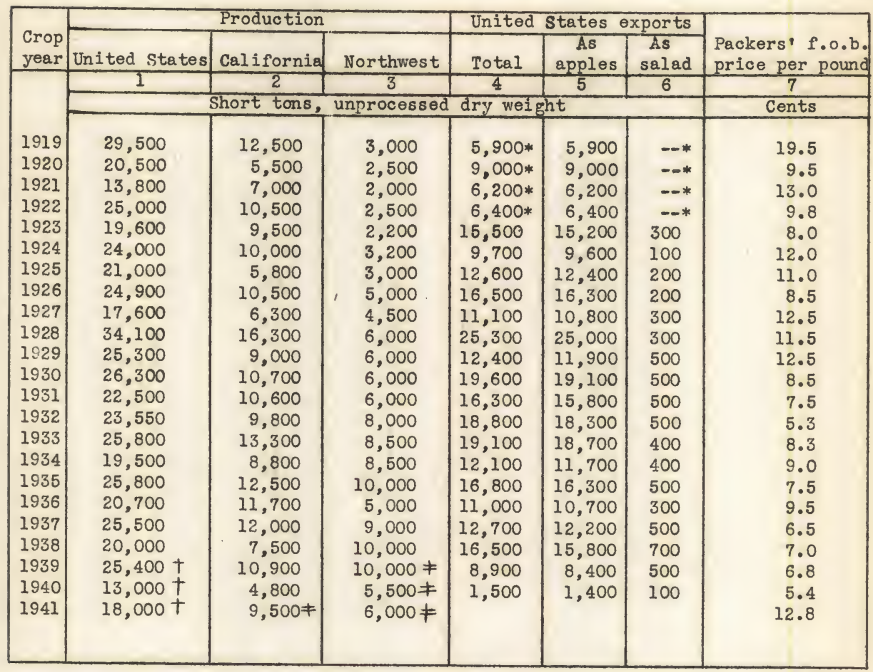

* Apple exports in salad are excluded in 1919-1922 as data not available. Coast.

+ Includes very rough estimates of production in states other than pacific

F Preliminary estimates.

Sources of data: Compiled by S. W. Shear, Giannini Foundation of Agricultural Tconomics, University of California.

Cols. 1-3: Trade estimates except California data based largely on packer receipts as reported by the Dried Fruit Association of California.

Cols. 4-6: Exports of dried apples and 7 per cent of exports of dried fruit salad, years beginning July 1. Based on official data of U. S. Bureau Foreign and Domestic Commerce, except salad in 1923-1929 estimated by S. W. Shear based largely on inspections by Dried Fruit Association of California.

Col. 7: Approximate f.o.b. California packers' selling prices for choice as reported in California Fruit News, August to December, inclusive. 


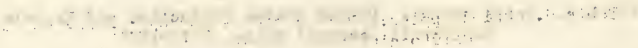

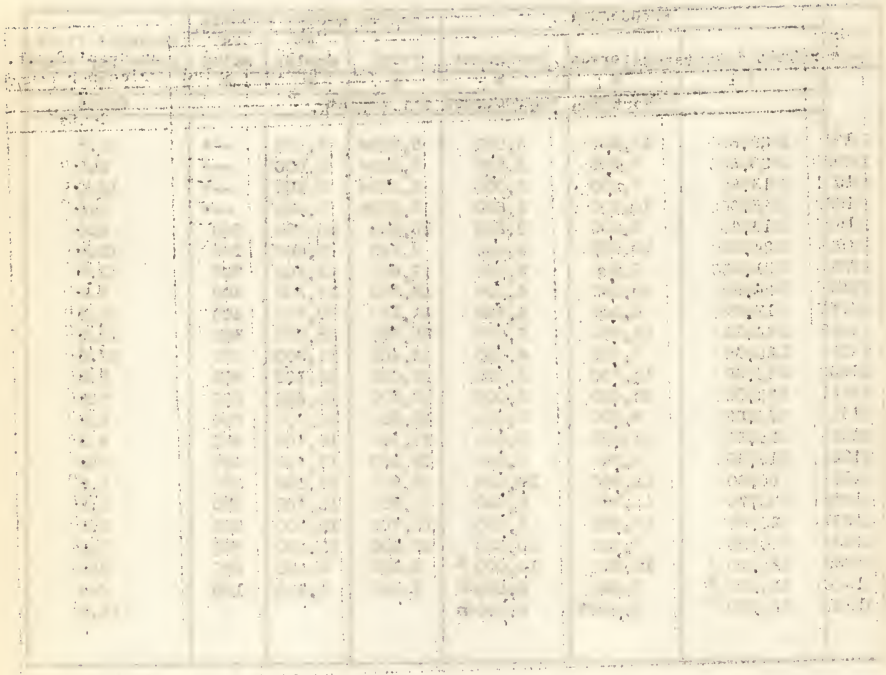

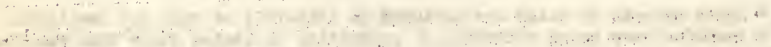

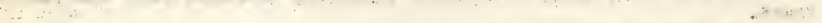

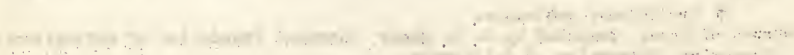

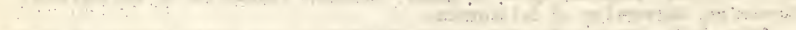

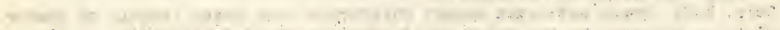

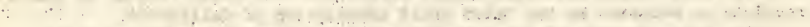

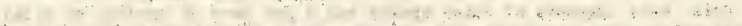

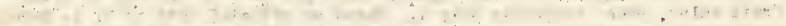

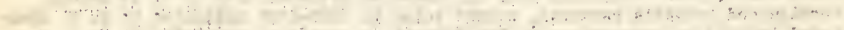

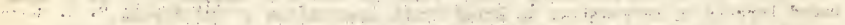

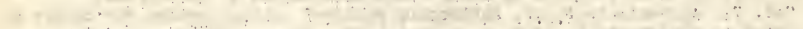
$\cdots+\cdots+\cdots+\cdots+\cdots+\cdots+\cdots+\cdots+\cdots$ 
APPLES

January, 1942

Table 7. Dried Apples:* United States Domestic Exports of Countries of Destination, Years Beginning July 1, 1930-1940

\begin{tabular}{|c|c|c|c|c|c|c|c|c|c|c|c|}
\hline $\begin{array}{l}\text { Country of } \\
\text { destination }\end{array}$ & $1930-31$ & $1931-32$ & $1932-33$ & $1933-34$ & $1934-35$ & $1935-36$ & $1936-37$ & $1937-38$ & $1938-39$ & $1939-40^{\top}$ & $1940-41^{\neq}$ \\
\hline & \multicolumn{11}{|c|}{ Short tons, net declared dry export weight } \\
\hline Total exports & 19,061 & 15,778 & 18,301 & 18,670 & 11,748 & 16,285 & 10,639 & 12,218 & 15,815 & 8,370 & $876 \neq$ \\
\hline Europe, total & 18,797 & 15,496 & 18,010 & 18,382 & 11,320 & 15,668 & 10,062 & 11,624 & 15,171 & 7,653 & 566 \\
\hline United Kingdom & 878 & 1,099 & 683 & 552 & 671 & 1,264 & 518 & 1,140 & 1,170 & $1,5 \perp 9$ & 0 \\
\hline Other & 17,919 & 14,397 & 17,327 & 17,830 & 10,649 & 14,404 & 9,544 & 10,484 & 14,001 & 6,134 & 566 \\
\hline France & 1,478 & 1,348 & 1,041 & 1,326 & 1,197 & 2,648 & 3,762 & 2,140 & 2,630 & 218 & 0 \\
\hline Netherlands & 4,382 & 4,077 & 3,784 & 3,613 & 2,478 & 4,678 & 2,667 & 4,655 & 5,948 & 3,294 & 0 \\
\hline Belgium & 535 & 183 & 210 & 224 & 152 & 262 & 432 & 382 & 667 & 34 & 0 \\
\hline Norway & 324 & 332 & 366 & 209 & 273 & 298 & 275 & 370 & 378 & 275 & 0 \\
\hline Sweden & 923 & 1,250 & 2,022 & 1,519 & 1,240 & 1,933 & 1,189 & 1,364 & 1,907 & 1,543 & 404 \\
\hline Denmark & 580 & 714 & 602 & 424 & 136 & 33 & 38 & 128 & 718 & 236 & $\oint$ \\
\hline Germany 9 & 9,235 & 6,028 & 8,918 & 9,986 & 4,704 & 3,333 & 799 & 781 & 1,053 & 10 & 0 \\
\hline Other & 462 & 465 & 384 & 529 & 469 & 1,219 & 382 & 664 & 700 & 524 & 162 \\
\hline Except Europe, total & 264 & 282 & 291 & 288 & 428 & 617 & 577 & 594 & 644 & 717 & 310 \\
\hline Canada & 11 & 34 & 41 & 7 & 21 & 142 & 134 & 34 & 59 & 47 & 14 \\
\hline Other & 253 & 248 & 250 & 281 & 407 & 475 & 443 & 560 & 585 & 670 & 296 \\
\hline
\end{tabular}

* Do not include exports of dried apples in dried fruit compote or salad.

+ Preliminary.

F Nine months only, July 1940-March 1941 as data by countries of destination not available for later months.

Total exports for July-June were 1,334 tons.

$\$$ Less than 0.5 of a ton.

9 Data for Germany include Austria beginning May 6, 1938, and parts of Czechoslovakia and Memel acquired since then.

Source of data: Compiled by S. W. Shear, Giannini Foundation of Agricultural Economics, University of California, from official data of U. S. Bureau of Foreign and Domestic Commerce as compiled largely by Office of Foreign Agricultural Relations, U. S. Dept. Agr. and U. S. Dept. Agr., Agricultural Statistics, 1938, p. 395. 


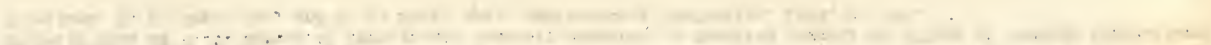

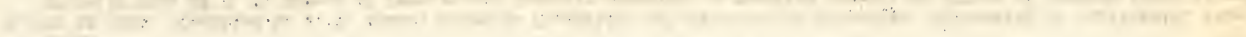

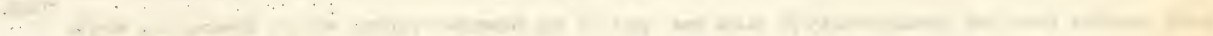
$\therefore+\ldots, \cdots, \cdots, \cdots, \cdots$

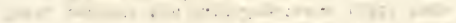

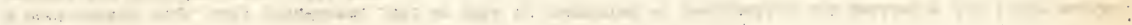

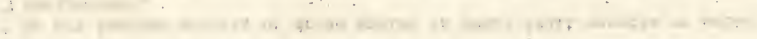

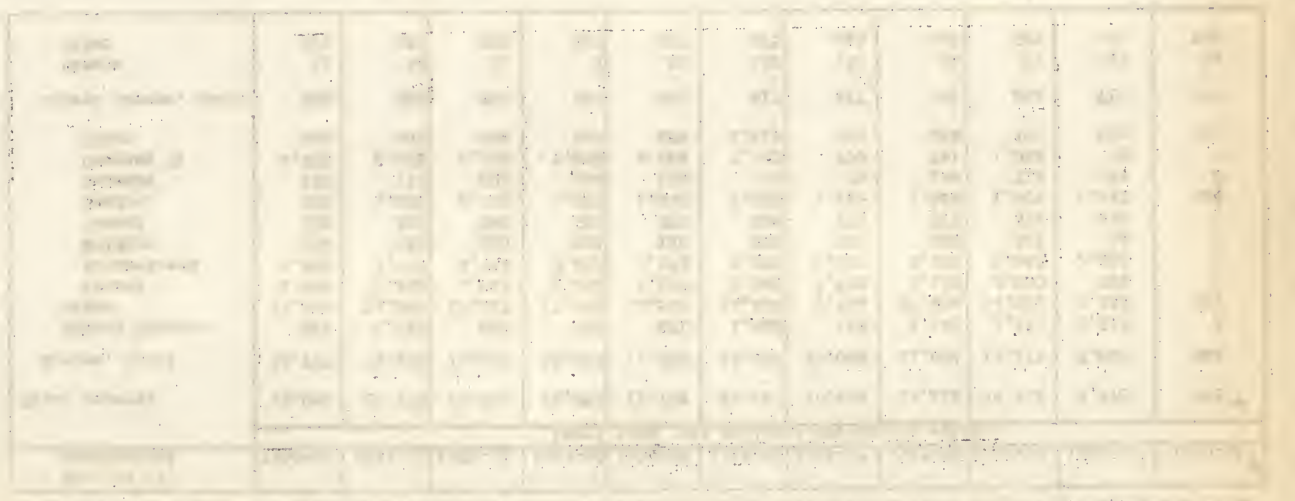

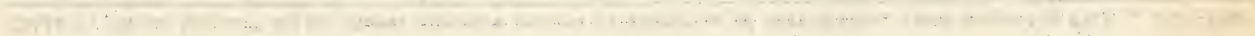


University of California, College of Agriculture

Agricultural Experiment Station, Berkeley, January, 1942

DECIDUOUS FRUIT STITISTICS

APRICO'SS

Table 1. Washington Production and California Production, Condition, Yield, Acreage, and Farm Value of hpricots, 1919-1941

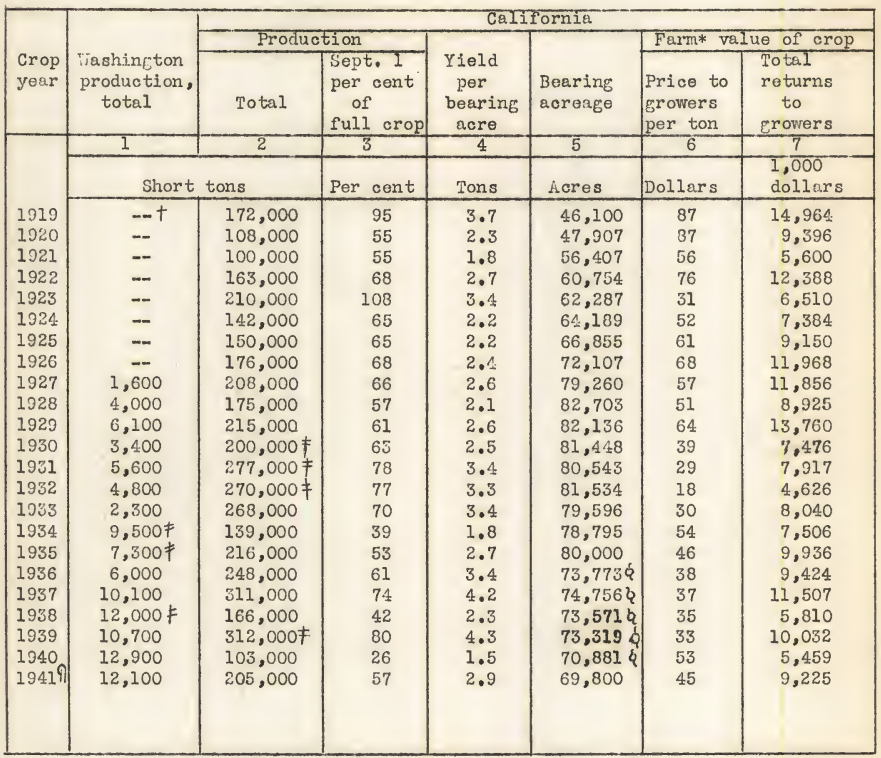

* Growors' returns for naked fruit delivered at crovers' first delivery point.

† Doshes indicate data not available.

F Includes unharvested tonnace: California -- 1930, 8,300; 1931, 4,000; 1932, 13,000 ; and 1939, 8,000. Washington -- 1954, 200; 1955, 700; and 1938, 2,300.

\$ Ionbearin: acres: 1956, 4,836; 1937, 5,044; 1938, 5,388; 1039, 5,052; and $1940,4,732$.

9) Prelininary estirates.

Sources of data: Compiled by S. T. Shear, Giannini Foumdation of Agricultural

Economics, University of California.

Col. 1: U. S. Dept. AEr., Agricultural Statistics, 1941, p. 193, and reports of the United States Crop Reporting Service.

Cols. 2, 3, 5, and 6: From California Cooperstive Crop Reporting Service.

Cols. 4 and 7: Calculated from other data in this table. 



\section{F.PRICOTS}

fable 2

California Apricots: Ilarvested Production, Utilization, and

Fresh F.O.B. Price, 1921-1941

\begin{tabular}{|c|c|c|c|c|c|c|c|}
\hline \multirow{3}{*}{$\begin{array}{l}\text { Crop } \\
\text { year }\end{array}$} & \multirow{3}{*}{$\begin{array}{c}\text { Production, } \\
\text { harvested }\end{array}$} & \multicolumn{5}{|c|}{ Utilization } & \multirow{3}{*}{$\begin{array}{c}\text { Fresh f.o.b. } \\
\text { price Royal, } \\
\text { per crate }\end{array}$} \\
\hline & & \multirow[b]{2}{*}{ Dried } & \multirow[b]{2}{*}{ Canned } & \multicolumn{3}{|c|}{ Consumed fresh } & \\
\hline & & & & Total & In state & $\begin{array}{r}\text { Out of } \\
\text { state }\end{array}$ & \\
\hline & 1 & 2 & 3 & 4 & 5 & 6 & 7 \\
\hline & \multicolumn{6}{|c|}{ Short tons, fresh weicht } & Dollars \\
\hline 1921 & 100,000 & 66,000 & 20,100 & 13,900 & 10,300 & 3,600 & 1.28 \\
\hline 1922 & 163,000 & 85,000 & 62,000 & 16,000 & 13,600 & 2,400 & 1.44 \\
\hline 1923 & 210,000 & 165,000 & 27,000 & 18,000 & 9,600 & 8,400 & 1.05 \\
\hline 1924 & 142,000 & 88,000 & 35,000 & 19,000 & 13,000 & 6,000 & 1.12 \\
\hline 1925 & 150,000 & 99,000 & 37,600 & 13,400 & 8,600 & 4,800 & 1.19 \\
\hline 1926 & 176,000 & 103,400 & 58,800 & 13,800 & 10,700 & 3,100 & 1.45 \\
\hline 1927 & 208,000 & 137,500 & 54,000 & 16,500 & 10,900 & 5,600 & 1.23 \\
\hline 1928 & 175,000 & 121,700 & 36,300 & 17,000 & 12,200 & 4,800 & 1.55 \\
\hline 1929 & 215,000 & 121,500 & 73,100 & 20,400 & 15,000 & 5,400 & 1.30 \\
\hline 1930 & 191,700 & 131,000 & 35,500 & 25,200 & 17,800 & 7,400 & 1.22 \\
\hline 1931 & 273,000 & 207,000 & 36,500 & 29,500 & 17,100 & 12,100 & 0.56 \\
\hline 1932 & 257,000 & 194,000 & 32,800 & 30,200 & 18,400 & 11,800 & 0.63 \\
\hline 1933 & 268,000 & 206,000 & 43,900 & 18,100 & 11,100 & 7,000 & 0.96 \\
\hline 1934 & 139,000 & 92,400 & 32,900 & 13,700 & 9,400 & 4,300 & 1.08 \\
\hline 1935 & 216,000 & 142,000 & 57,500 & 16,500 & 11,500 & 5,000 & 0.82 \\
\hline 1936 & 248,000 & 177,200 & 52,700 & 18,100 & 11,400 & 6,700 & 1.10 \\
\hline 1937 & 311,000 & 189,000 & 101,000 & 21,000 & 13,700 & 7,300 & 1.09 \\
\hline 1938 & 166,000 & 117,600 & 28,100 & 20,300 & $\$ 4,700$ & 5,600 & 0.76 \\
\hline 1939 & 304,000 & 225,500 & 55,700 & 22,800 & 15,200 & 7,600 & 1.08 \\
\hline 1940 & 103,000 & 58,300 & 30,000 & 14,700 & 10,400 & 4,300 & 1.31 \\
\hline 1941 & $205,000 *$ & $115,500 *$ & $65,600 *$ & $23,900 *$ & $17,700 *$ & $6,200 *$ & 0.95 \\
\hline & & & & & & & \\
\hline
\end{tabular}

* Preliminary; utilization data are unofficial estinates.

Sources of data: Compiled by S. i. Shear, Giannini Foundation of kgricultural Economies, University of Califoriia.

Cols. 1-4: From California Crop Reports, excopt 1922 and 1941, cols. 2-4. Dried converted to fresh at ratio of 5.5 to 1 . Cases of $24110.2 \frac{1}{2}$ cans converted a. 55 cases per ton, except 1939-1940 at 60 and 1941 ut 62 .

Col. 5: Col. 4 minus col. 6 .

Col. 6: Based on interstate rail shipments, compiled since 1926 by FederalState liarlzet liews Service; data for recent yeurs from Californic Crop Reports, excent 1941.

Col. 7: Season's woighted average delivered-auction wice in New York City and Chicago minus freight, refriferation, und 7 per cent comsission. 

APR ICOTS

rable 3

Dried „pricots: California Production, Exports, and

Packers' F.0.B. Prices, 1921-1941

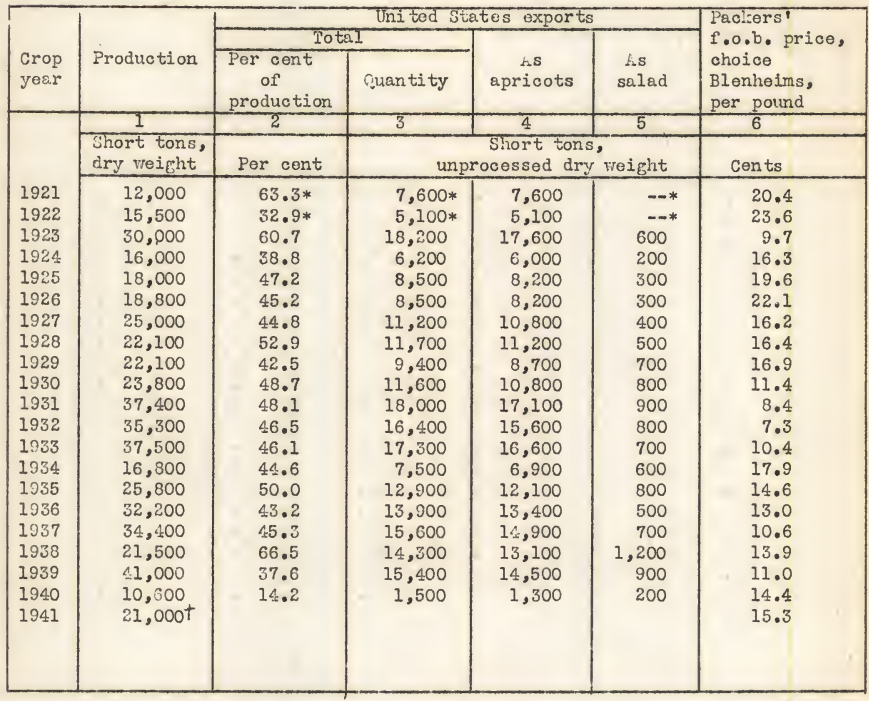

* Apricot exports in salad are excluded in 1921 and 1922 es data not availuble. + Rough trade estimate subject to considerable revision.

Sources of data: Compiled by S. $\because$. Shear, Giannini Foundation of Agricultural Economics, University of California.

Col. 1: Based upon reports of the Dried Fruit Association of California and of the California Cooperative Crop Reporting Service.

Cols. 3-5: Exports of dried aprioots and $121 / 3$ per cent of exports of dried fruit salad, years beginning July 1 . Based on official data of U.S. Bur. For, and Dom. Com, except salad, 1923-1929, estimated by S. it. Shear largely from inspections by Jried Fruit fssociation of California.

Col. 6: Simple average of packers' f.o.b. California quotations (middle of range) on choice Blenheims in 25-pound boxes for six months July 1 December 31 , compiled from California Fruit ilews. 



\section{Table 4}

Dried Apricots: United States Domestic Exports by Countries of Destination, Years Beginning July 1, 1930-1940

\begin{tabular}{|c|c|c|c|c|c|c|c|c|c|c|c|}
\hline $\begin{array}{l}\text { Country of } \\
\text { destination }\end{array}$ & $1930-31$ & $1931-32$ & $1932-33$ & $1233-34$ & $13 \geq 4-35$ & $1935-36$ & $1936-37$ & $1937-38$ & $1938-39$ & $\begin{array}{r}+1939-40^{+} \\
\end{array}$ & $1940-41^{\ddagger}$ \\
\hline \multirow[b]{3}{*}{ Total exports } & 1 & 2 & 3 & 4 & 5 & 6 & 7 & 8 & 9 & 10 & 11 \\
\hline & \multicolumn{11}{|c|}{ Short tons, net declared dry expori weinh } \\
\hline & 11,823 & 18,811 & 17,135 & 18,308 & 7,597 & 13,400 & 14,682 & 16,425 & 14,420 & 15,961 & $1,105^{\ddagger}$ \\
\hline Europe, total & 10,995 & 17,471 & 15,672 & 17,040 & 7,067 & 12,142 & 13,343 & 14,527 & 13,300 & 14,099 & 79 \\
\hline United Kingdom & 621 & 1,394 & 1,585 & 1,303 & 514 & 1,468 & 1,591 & 1,845 & 1,813 & 2,487 & 4 \\
\hline Other & 10,374 & 16,077 & 14,087 & 15,737 & 6,553 & 10,674 & 11,752 & 12,682 & 11,487 & 11,612 & 75 \\
\hline France & 1,259 & 3,570 & 4,125 & 4,414 & 2,969 & 5,357 & 6,434 & 6,273 & 3,848 & 3,149 & 0 \\
\hline lietherlands & 1,467 & 1,956 & 1,406 & 1,596 & 814 & 1,270 & 1,467 & 1,770 & 1,410 & 2,051 & 0 \\
\hline Belgium & 966 & 1,003 & 883 & 1,100 & 654 & 928 & 1,365 & 1,483 & 1,544 & 1,010 & 0 \\
\hline Noriay & 393 & 695 & 566 & 358 & 300 & 451 & 484 & 601 & 375 & 883 & 0 \\
\hline Sweren & 417 & 575 & 606 & 476 & 265 & 653 & 733 & 775 & 725 & 1,125 & 51 \\
\hline Denmark & 1,145 & 1,686 & 726 & 887 & 67 & 46 & 40 & 188 & 1,658 & 2,264 & 0 \\
\hline Germany $\mathfrak{\&}$ & 4,347 & 5,899 & 5,395 & $6,2 \approx 5$ & 948 & 1,135 & 614 & 747 & 632 & 34 & 0 \\
\hline Other & 410 & 693 & 380 & 681 & 536 & 834 & 615 & 845 & 1,295 & 1,096 & 24 \\
\hline Ex:cept iurope & 828 & 1,340 & 1,463 & 1,268 & 530 & 1,258 & 1,339 & 1,898 & 1,120 & 1,862 & 1,026 \\
\hline Canada & 518 & 917 & 971 & 766 & 189 & 734 & 740 & 1,096 & 654 & 1,173 & 709 \\
\hline Other & 310 & 423 & 492 & 502 & 341 & 524 & 599 & 802 & 466 & 689 & 317 \\
\hline
\end{tabular}

* Do rot include exports of dried apricots in dried fruit compote or salad.

T Preliminary

F Nine months only, July 1940-:arch 1941 as data by countries of destinstion not available for later months. Total exports for July-June were 1,454 tons.

\& Data for Germany include Austrie beginning kay 6, 1938, and certsin parts of Czechoslovakia and themel acquired by Germeny since then.

Source of data: Compiled by S. W. Shear, Giannini Foundation of Agricultural Economics, University of California, from official data of the U. S. Bureau of Foreign and Domestic Commerce, largely compiled directly. 
.

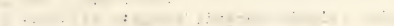

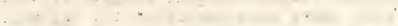

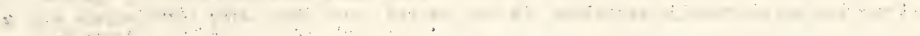

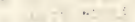

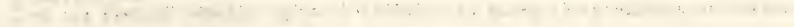
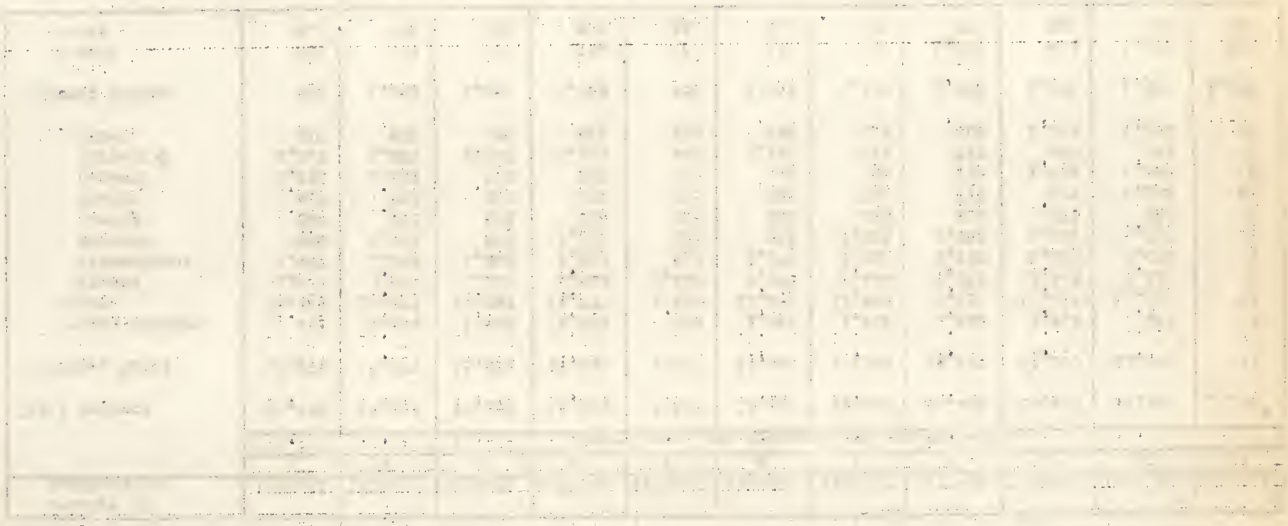

:...

(............. 
Table 5. California Canned Apricots: Pack, Cerryover, Shipments, Exports and Price, 1921-1941

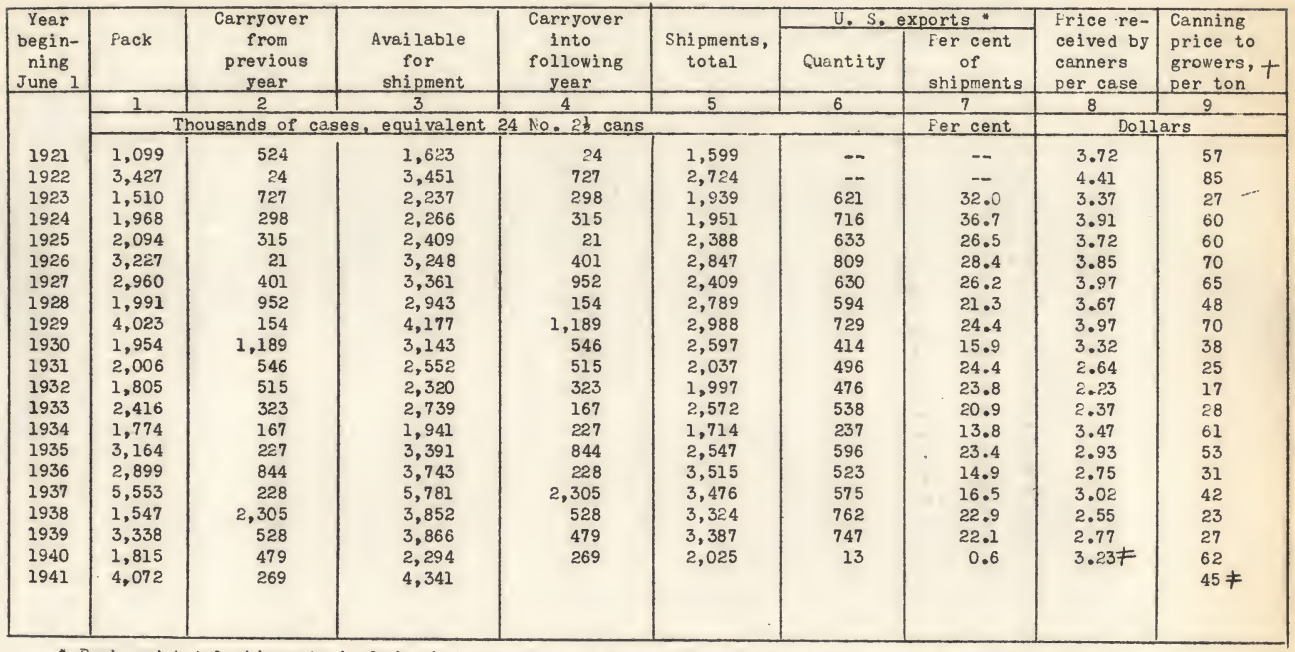

* Pack and total shipments include, but exports exclude, apricots repacked into salad and cocktail.

TGrowers' returns for noked fruit delivered at grouers' first delivery point. $\quad$ Prelilinary estimates. Sources of data: Compiled by Giannini Foundation of Agricultural Economics, University of California.

Cols. 1, 2, and 4: Based upon mimeographed releases of the Canners League of California.

Col. 6: U. S. Monthly Summary of Foreign Commerce, converted at 45 pounds per case of 24 No. $2 \frac{1}{2}$ cans.

Col. 8: Weighted average prices of all grades and sizes of cans on an unadvertised basis and excluding trade

discounts, as compiled by H. R. Wellman, from canners' reports.

Col. 9: From California Cooperative Crop Reporting Service, except 1941 is a preliminary trade estinate. 
-

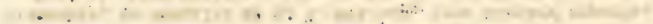

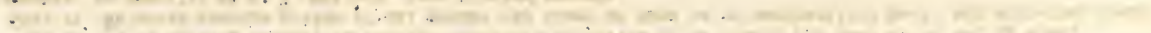

.

Th +

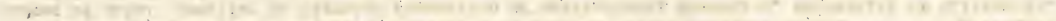

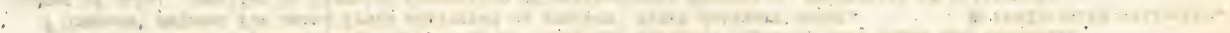

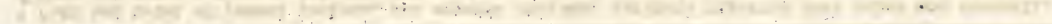

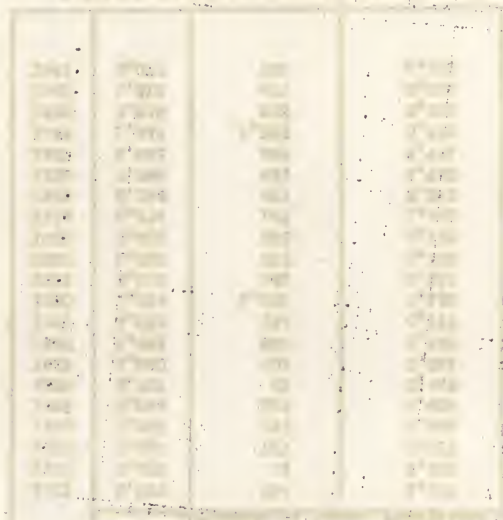
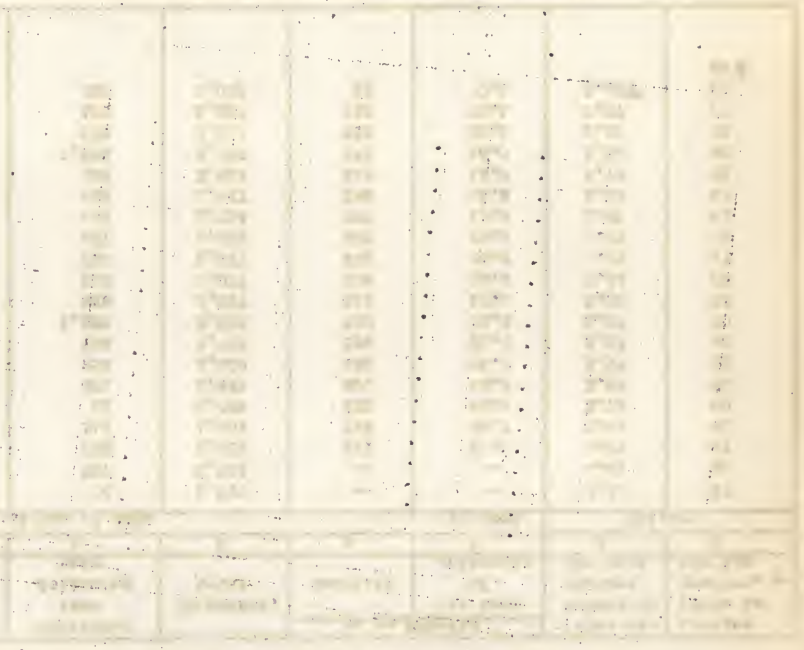

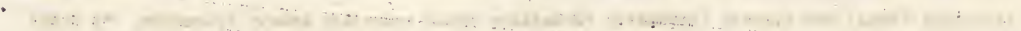




\section{APRICOTS}

Table 6

California Canned Apricots: Pack, Carryover, and Hovement, 1931-1941

\begin{tabular}{|c|c|c|c|c|c|c|c|c|c|}
\hline \multirow{3}{*}{$\begin{array}{l}\text { Year } \\
\text { begin- } \\
\text { ning } \\
\text { June } 1\end{array}$} & \multirow{3}{*}{$\begin{array}{l}\text { Supply } \\
\text { June } 1\end{array}$} & \multirow[b]{3}{*}{ Pack } & \multicolumn{3}{|c|}{$\begin{array}{l}\text { Carryover, unshipped } \\
\text { (i.e. sold and unsold) }\end{array}$} & \multicolumn{4}{|c|}{ Hovement } \\
\hline & & & \multirow[b]{2}{*}{$\begin{array}{l}\text { June } 1 \\
\text { of year } \\
\text { indi- } \\
\text { cated }\end{array}$} & \multirow[b]{2}{*}{$\begin{array}{l}\text { Decenn- } \\
\text { ber } 31\end{array}$} & \multirow[b]{2}{*}{$\begin{array}{l}\text { June } 1 \\
\text { of fol- } \\
\text { lowing } \\
\text { rear }\end{array}$} & \multicolumn{2}{|c|}{ June-December } & \multirow[b]{2}{*}{$\begin{array}{l}\text { Jan- } \\
\text { Ilay }\end{array}$} & \multirow[b]{2}{*}{$\begin{array}{c}\text { Season's } \\
\text { total } \\
\text { June- } \\
\text { Ilay }\end{array}$} \\
\hline & & & & & & $\begin{array}{l}\text { Per cont } \\
\text { of } \\
\text { season's } \\
\text { total }\end{array}$ & Quantity & & \\
\hline \multirow{4}{*}{$\begin{array}{l}1931 \\
1932\end{array}$} & 1 & 2 & 3 & 4 & 5 & 6 & 7 & 8 & 9 \\
\hline & \multicolumn{5}{|c|}{ Thousands of cases* } & Per cent & \multicolumn{3}{|c|}{ Thousends of cases* } \\
\hline & 2,552 & 2,006 & 546 & 1,071 & 515 & 73 & 1,481 & 556 & 2.037 \\
\hline & 2,320 & 1,805 & 515 & 910 & 323 & 71 & $\begin{array}{l}1,401 \\
1,410\end{array}$ & $\begin{array}{l}500 \\
587\end{array}$ & $\begin{array}{l}2,001 \\
1,997\end{array}$ \\
\hline 1933 & 2,739 & 2,416 & 323 & 733 & 167 & 78 & 2,006 & 566 & 2,572 \\
\hline 1934 & 1,941 & 1,774 & 167 & 592 & 227 & 79 & 1,349 & 365 & 1,714 \\
\hline 1935 & 3,391 & 3,164 & 227 & 1,581 & 844 & 71 & 1,810 & 737 & 2,547 \\
\hline 1936 & 3,743 & 2,899 & 844 & 1,080 & 228 & 76 & 2,663 & 852 & 3,515 \\
\hline 1937 & 5,781 & 5,553 & 228 & 3,202 & 2,305 & 74 & 2,579 & 897 & 3,476 \\
\hline 1938 & 3,852 & 1,547 & 2,305 & 1,509 & 528 & 70 & 2,343 & 981 & 3,324 \\
\hline 1939 & 3,866 & 3,338 & 528 & 1,266 & 479 & 77 & 2,600 & 787 & 3,387 \\
\hline 1940 & 2,294 & 1,815 & 479 & 878 & 269 & 70 & 1,416 & 609 & 2,025 \\
\hline 1941 & 4,341 & 4,072 & 269 & 1,294 & & & 3,047 & & \\
\hline & & & & & & & & & \\
\hline & & & & & & & & & \\
\hline
\end{tabular}

* Equivalent casos of 24 IIo. $2 \frac{2}{2}$ oans.

f As stoclis on hand December 31, 1941, exclude goods sold to the Eovernment the movoment for June-December, 1941, includessales to the government noved and not moved, and so stocks are relatively too low and the rovement too hich as compared with data for other years.

Source of data:

Compiled by S. T. Shear, Giannini Foundation of Agricultural Economics, University of California, largely on the basis of data from mineographed releases of the Conners League of California. 


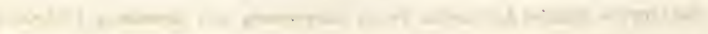

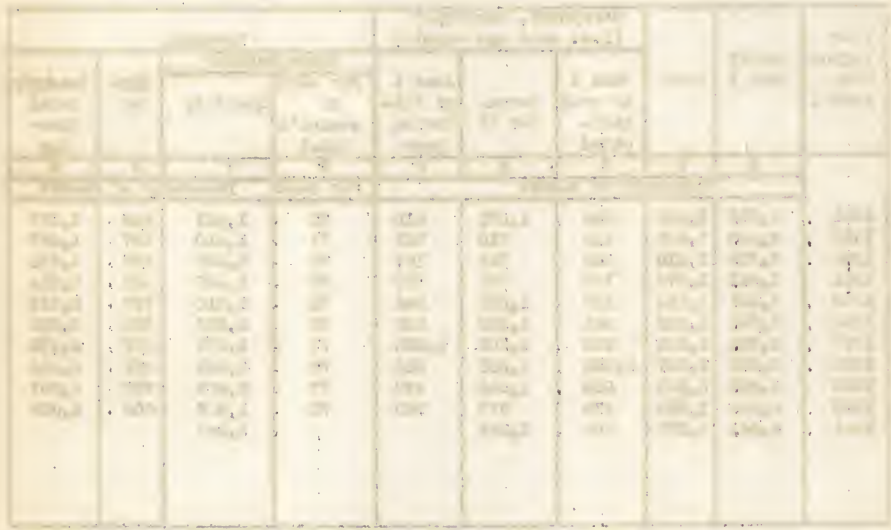

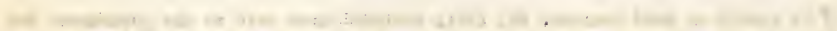

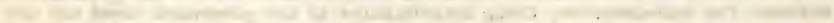

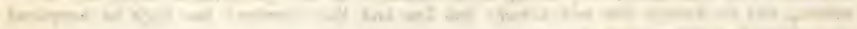
(1)

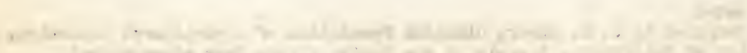

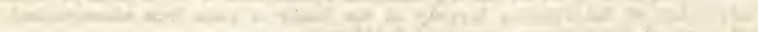

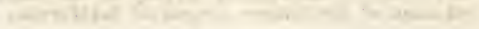


University of cal ifornia, College- of Agriculture

Agricultural Experiment Station, Berkeley, January, 1942

DECIDUOUS FRUIT STATISTICS

CHERRIES

Table 1

California Cherries: Bearing Acreage, Production, Condition Yield, and Farm Value, 1919-1941

\begin{tabular}{|c|c|c|c|c|c|c|}
\hline \multirow[b]{2}{*}{$\begin{array}{l}\text { Crop } \\
\text { year }\end{array}$} & \multirow[b]{2}{*}{$\begin{array}{l}\text { Bearing } \\
\text { acreage }\end{array}$} & \multicolumn{2}{|c|}{ Production } & \multirow[b]{2}{*}{$\begin{array}{c}\text { Yield per } \\
\text { bearing } \\
\text { acre }\end{array}$} & \multicolumn{2}{|c|}{ Farm*value of crop } \\
\hline & & Total & $\begin{array}{l}\text { July } 1 \\
\text { per cent of } \\
\text { full crop }\end{array}$ & & $\begin{array}{l}\text { Price to } \\
\text { growers } \\
\text { per ton }\end{array}$ & $\begin{array}{l}\text { Total } \\
\text { returns to } \\
\text { growers }\end{array}$ \\
\hline & 1 & 2 & 3 & 4 & 5 & 6 \\
\hline & Acres & Short tons & Per cent & Short tons & Dollars & 1,000 dollars \\
\hline 1919 & 8,750 & 12,400 & 82 & 1.4 & 150 & 1,860 \\
\hline 1920 & 8,884 & 17,500 & 87 & 2.0 & 200 & 3,500 \\
\hline 1921 & 8,758 & 13,000 & 75 & 1.5 & 125 & 1,625 \\
\hline 1922 & 9,317 & 17,000 & 70 & 1.8 & 180 & 3,060 \\
\hline 1923 & 9,646 & 19,000 & 81 & 2.0 & 160 & 3,040 \\
\hline 1924 & 9,981 & 13,500 & 65 & 1.4 & 140 & 1,890 \\
\hline 1925 & 10,433 & 12,000 & 50 & 1.2 & 160 & 1,920 \\
\hline 1926 & 10,828 & 20,000 & 72 & 1.8 & 180 & 3,600 \\
\hline 1927 & 10,554 & 12,000 & 45 & 1.1 & 180 & 2,160 \\
\hline 1928 & 11,606 & 16,600 & 63 & 1.4 & 150 & 2,490 \\
\hline 1929 & 11,984 & 16,300 & 53 & 1.4 & 190 & 3,097 \\
\hline 1930 & 12,555 & 17,500 & 61 & 1.4 & 148 & 2,590 \\
\hline 1931 & 13,147 & $23,000+$ & 77 & 1.7 & 93 & 1,860 \\
\hline 1932 & 13,621 & $18,500^{\circ}$ & 54 & 1.4 & 60 & 960 \\
\hline 1933 & 14,004 & $25,300^{+}$ & 73 & 1.8 & 66 & 1,643 \\
\hline 1934 & 14,551 & 17,000 & 49 & 1.2 & 90 & 1,530 \\
\hline 1935 & 14,200 & 15,000 & 49 & 1.1 & 125 & 1,875 \\
\hline 1936 & $14,532 \neq$ & 23,000 & 63 & 1.6 & 102 & 2,346 \\
\hline 1937 & $15,228^{F}$ & 21,600 & 54 & 1.4 & 170 & 3,672 \\
\hline 1938 & $15,211^{\ddagger}$ & $30,000+$ & 72 & 2.0 & 84 & 2,117 \\
\hline 1939 & $14,881 \%$ & $36,000^{+}$ & 82 & 2.4 & 79 & 2,607 \\
\hline 1940 & $13,919^{\mathrm{T}}$ & 11,000 & 31 & 0.8 & 141 & 1,551 \\
\hline $1941 \oint$ & 13,600 & 20,200 & 53 & 1.5 & 127 & 2,565 \\
\hline & & & & & & \\
\hline
\end{tabular}

*Grewers' returns for naked fruit delivered at growers' first delivery point.

TIncludes unharvested tonnage: $1931 ; 3,000 ; 1932,2,500 ; 1933,400$; 1938, 4,800 ; and $1939,3,000$.

F Nonbearing acres; 1936, 2,200; 1937, 2,018; 1938, 1,669; 1939, 1,171; and $1940,1,110$.

y Preliminary estimates.

Sources of data: Compiled by S. Wo Shear, Giannini Foundation of Agricultural

Economics, University of California from reports of the California Cooperative

Crop Repar ting Service, except cols. 4 and 6 calculated. 
$\therefore$

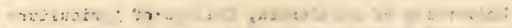

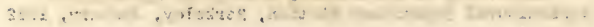

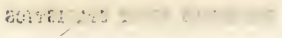

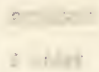

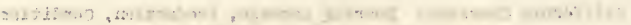
Dis.

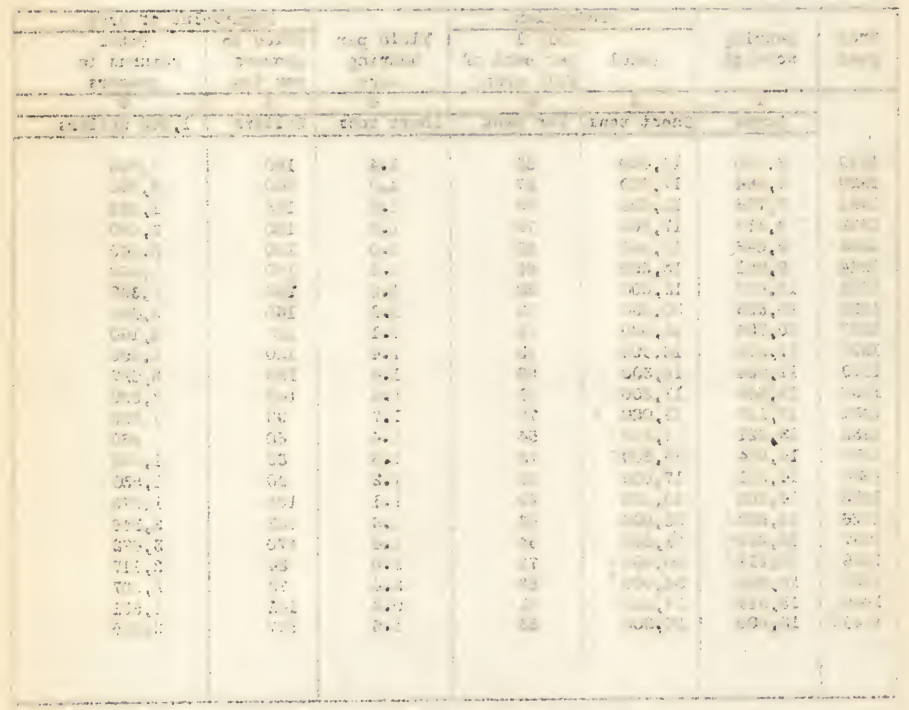

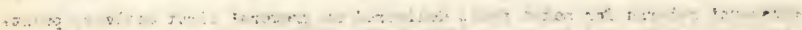
a

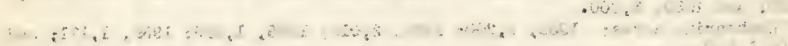
...

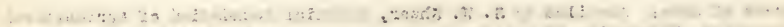

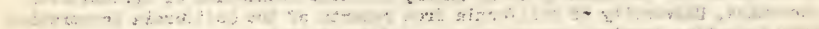

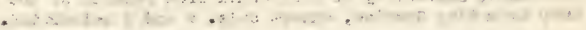


January, 1942

CHERRIES

Table 2. California Cherries: Production and Utilization, 1928-1941

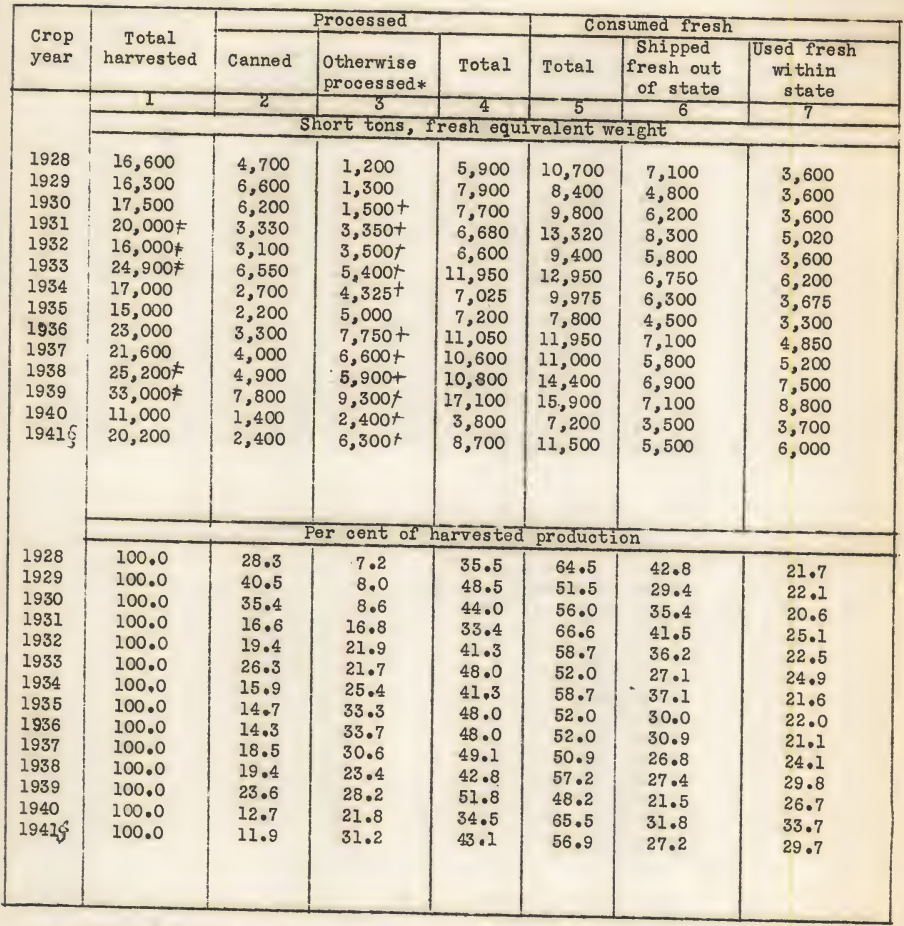

* Cherries "otherwise prncessed" are practioally all barrelled in brine. data on Royal Anns barr on the tonnage "otherwise processed" disagree slightly from f Additional unharvested in brine as shown in table 4, col. 7 . 4,800; and $1939,3,000$.

$\$$ Preliminary unofficial estimates except for total harvested.

Source of data: Compiled by S. W. Shear, Giannini Foundation of 2grioultural Economics, University of California, from California Crop Reporting Service, except utilization data for 1941 which are unofficial pro liminary estimatese 


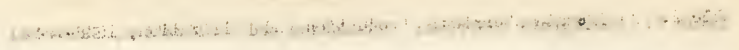

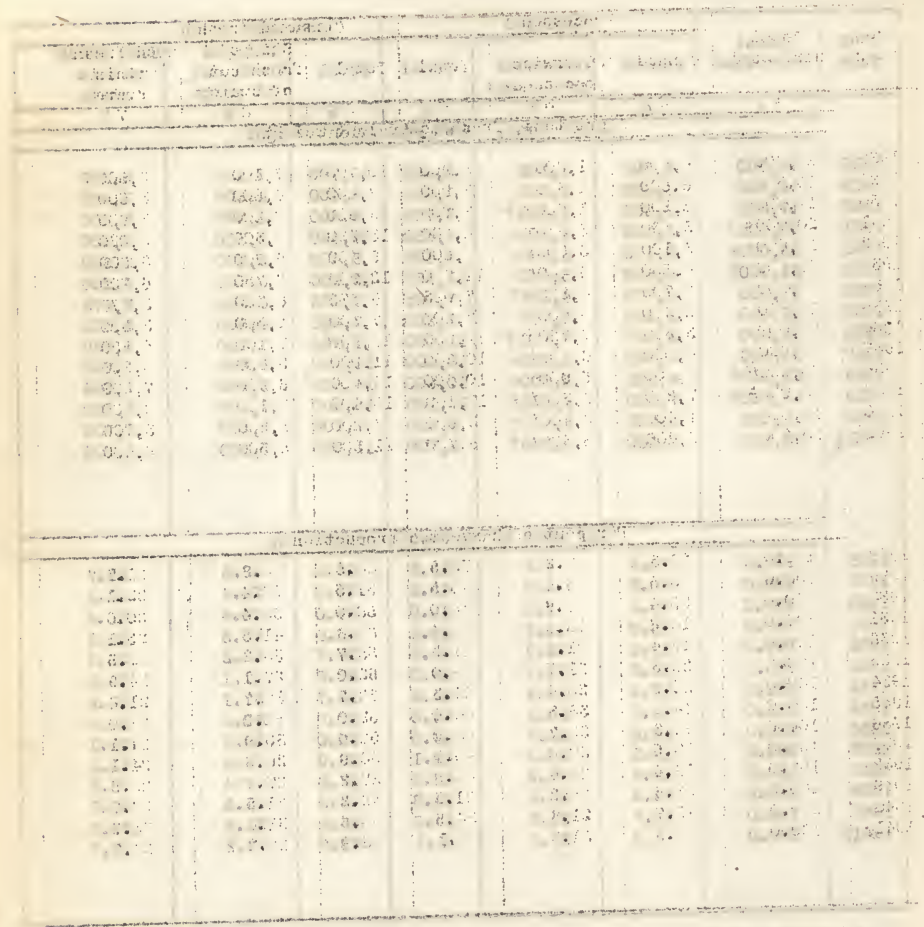

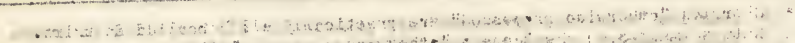

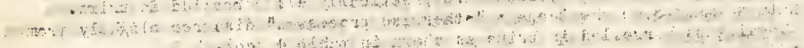
5C-

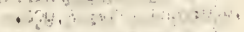
A.....

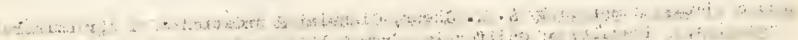

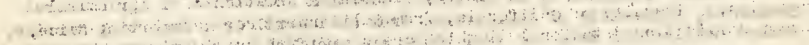

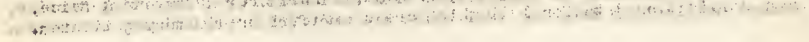




\section{CHERRIES}

Table 3

California Fresh cherries: Interstate shipments and Prices, 1922-1941

\begin{tabular}{|c|c|c|c|c|}
\hline \multirow[b]{2}{*}{ Crop year } & \multirow{2}{*}{\multicolumn{2}{|c|}{ Interstate shipments }} & \multicolumn{2}{|c|}{ Prioe per pound } \\
\hline & & & \multirow{3}{*}{\multicolumn{2}{|c|}{\begin{tabular}{c|c}
$\begin{array}{l}\text { New York } \\
\text { aution }\end{array}$ & $\begin{array}{c}\text { Calculated } \\
\rho_{0} 0 . b_{0}\end{array}$ \\
3 & 4 \\
\end{tabular}}} \\
\hline \multirow{22}{*}{$\begin{array}{l}1922 \\
1923 \\
1924 \\
1925 \\
1926 \\
1927 \\
1928 \\
1929 \\
1930 \\
1931 \\
1932 \\
1933 \\
1934 \\
1935 \\
1936 \\
1937 \\
1938 \\
1939 \\
1940 \\
1941\end{array}$} & 1 & 2 & & \\
\hline & Cars & Short tons & & \\
\hline & 510 & 4,080 & 25.5 & 17.7 \\
\hline & 600 & 4,800 & 26,4 & 18.6 \\
\hline & 600 & 4,800 & 22.0 & 14.6 \\
\hline & 520 & 4,160 & 22.4 & 15.4 \\
\hline & 750 & 6,000 & 24.0 & 17.0 \\
\hline & 579 & 4,600 & 25.1 & 28,1 \\
\hline & 898 & 7,100 & 19,3 & 12,7 \\
\hline & 604 & 4,800 & 28,0 & 20.7 \\
\hline & 774 & 6,200 & 23,4 & 16.6 \\
\hline & 1,034 & 8,300 & 15.8 & 8.9 \\
\hline & 728 & 5,800 & $15 \cdot 6$ & 8.8 \\
\hline & 842 & 6,750 & 12.8 & 6.8 \\
\hline & 787 & 6,300 & 11.0 & 5.2 \\
\hline & 502 & 4,500 & 15.4 & 9.3 \\
\hline & 790 & 7,100 & 13.0 & 7.0 \\
\hline & $645 *$ & 5,800 & $16 \cdot 6$ & 10.4 \\
\hline & $775 *$ & 6,900 & 11.4 & 5.6 \\
\hline & $792 *$ & 7,100 & 11.9 & 6.0 \\
\hline & $390 *$ & 3,500 & 16.8 & 10.6 \\
\hline & $535 *$ & 5,500 & 17.1 & 10.9 \\
\hline & & & & \\
\hline & & & & \\
\hline
\end{tabular}

* Inoludes mixed cars, mostly oherries of 17 oars in 1937, 37 cars in 1938, 62 oars in 1939,15 cars in 1940 , and 19 cars 1 n 1941.

Souroes of data: Compiled by S. W. Shear, Giannini Foundation of Agrioultural Eoonomios, University of California.

Col. I: 1922-1920, interstate shipments based upon ocmpilation of Paoifio Fruit Express, mimeographed form 878. 1927-1941, Federal-State Market News Service, mimeographed reports on interstate shipments of Califomia deoiduous-tree fruits.

Col. 2: 1922-1927, col. I converted at 8 tons per oar. 1928-1941 from table 2, col. 6 .

Col. 3: Prices are season's weighted averages of Tartarian, Bing, Royal Ann, Republican, and Chapman.

Col. 4: Caloulated by deduoting freight, refrigeration, and a 7 per cent sales cemmission from the auotion prioo in col. 3 . 


$$
\because: 5, i,+\infty
$$

$\ldots \ldots \ldots$

$$
\text { i. is is }
$$

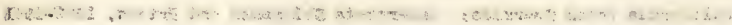

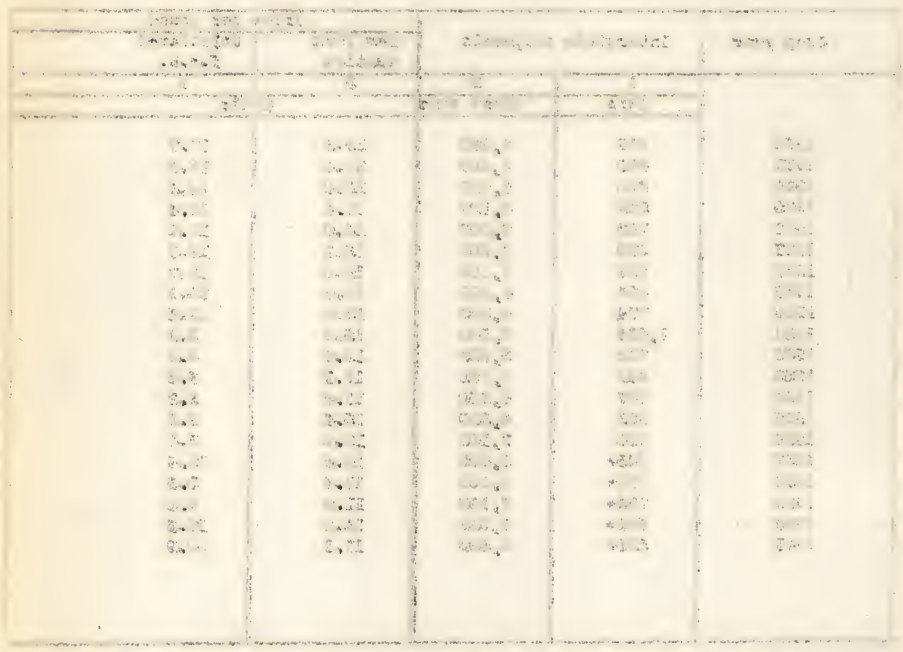

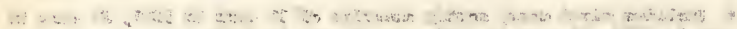

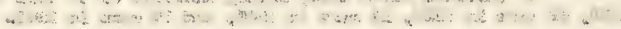

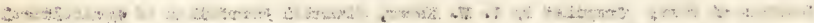
CH:

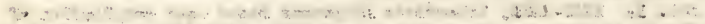

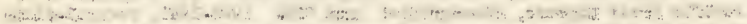

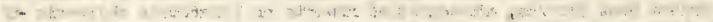

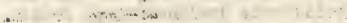

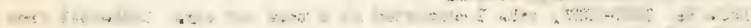

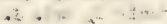

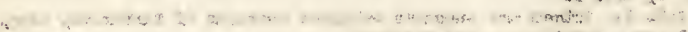

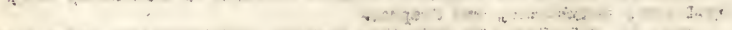

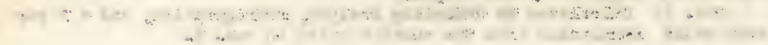


Table 4. Royal Anns Canned and Barrelled in Brine on the Pacific Coast and California Canning Price, 1922-194l

\begin{tabular}{|c|c|c|c|c|c|c|c|c|c|c|}
\hline \multirow{2}{*}{$\begin{array}{l}\text { Crop } \\
\text { year }\end{array}$} & \multirow{2}{*}{$\begin{array}{l}\text { Farm price } \\
\text { per pound }\end{array}$} & \multicolumn{3}{|c|}{ Pacific Coast } & \multicolumn{3}{|c|}{ Californie } & \multicolumn{3}{|c|}{ Pacific Northwest, } \\
\hline & & Total & Canned & Barrelled & Total & Canned & Barrelled & Total & Canned & Barrelled* \\
\hline & 1 & 2 & 3 & 4 & 5 & 6 & 7 & 8 & 9 & 10 \\
\hline & Cents & \multicolumn{9}{|c|}{ Short tons, fresh equivalent weight } \\
\hline 1922 & 10.4 & $T$ & 12,233 & $t$ & + & 8,000 & $t$ & $t$ & 4,233 & + \\
\hline 1923 & 9.8 & & 14,539 & & & 9,300 & & & 5,239 & \\
\hline 1924 & 5.9 & & 8,425 & & & 3,300 & & & 5,125 & \\
\hline 1925 & 8.8 & 9,883 & 7,958 & $1,925 \neq$ & 4,500 & 3,500 & $1,000 \neq$ & 5,383 & 4,458 & 925 \\
\hline 1926 & 9.5 & 20,083 & 17,932 & $2,151 \neq$ & 9,492 & 8,492 & 1,0007 & 10,591 & 9,440 & 1,151 \\
\hline 1927 & 8.3 & 9,490 & 7,170 & $2,320 \neq$ & 3,754 & 2,754 & $1,000 \neq$ & 5,736 & 4,416 & 1,320 \\
\hline 1928 & 7.9 & 14,437 & 12,182 & 2,255 & 5,635 & 4,465 & 1,170 & 8,802 & 7,717 & 1,085 \\
\hline 1929 & 9.8 & 15,602 & 13,120 & 2,482 & 7,690 & 6,368 & 1,322 & 7,912 & 6,752 & 1,160 \\
\hline 1930 & 7.4 & 18,370 & 14,750 & 3,620 & 8,326 & 6,156 & 2,170 & 10,044 & 8,594 & 1,450 \\
\hline 1931 & 4.0 & 10,763 & $4,996 \grave{Q}$ & 5,767 & 6,825 & 3,330 & 3,495 & 3,938 & 1,6666 & 2,272 \\
\hline 1932 & 3.0 & 13,997 & $6,533 \delta$ & 7,464 & 6,258 & 3,067 & 3,191 & 7,739 & $3,466 \&$ & 4,273 \\
\hline 1933 & 4.0 & 24,697 & 12,711 oे & 11,986 & 11,542 & 6,542 & 5,000 & 13,155 & $6,169 \varepsilon$ & 6,986 \\
\hline 1934 & 5.0 & 14,906 & 6,9068 & 8,000 & 6,663 & 2,663 & 4,000 & 8,243 & $4,243 \phi$ & 4,000 \\
\hline 1935 & 6.6 & 18,261 & 7,361 ○ & 10,900 & 7,222 & 2,222 & 5,0009 & 11,039 & $5,139 \oint$ & 5,900 \\
\hline 1936 & 5.0 & 19,878 & 7,095 Qे & 12,783 & 10,217 & 3,342 & 6,875 & 9,661 & 3,7536 & 5,908 \\
\hline 1937 & 8.3 & 17,698 & 6,331 of & 11,367 & 9,765 & 3,998 & 5,767 & 7,933 & 2,3336 & 5,600 \\
\hline 1938 & 3.3 & 21,839 & $9,565 \oint$ & 12,274 & 10,158 & 4,899 & 5,259 & 11,681 & 4,6668 & 7,015 \\
\hline 1939 & 4.0 & 28,010 & 14,0858 & 13,925 & 16,360 & 7,813 & 8,547 & 11,650 & $6,272 \phi$ & 5,378 \\
\hline 1940 & 6.5 & 18,046 & 6,6390 & 11,407 & 3,330 & 1,398 & 1,932 & 14,716 & $5,241 \&$ & 9,475 \\
\hline $1941 /$ & 6.811 & 20,125 & 8,882 \& & 11,243 & 7,613 & 2,370 & 5,243 & 12,512 & $6,512 \oint$ & $6,000 /$ \\
\hline
\end{tabular}

* Northwest data include some brined black cherries for a few years before 1936.

+ No data on barrelled brine pack available before 1925, but it was very small.

\# Rough trade estimates as no reliable statistics available. \& No data given for Idaho $1931-1941$.

9/ Includes some brined black cherries. //1941 Northwest brined pack and California price are trade estimates.

Sources of data: Compiled by S. W. Shear, Giannini Foundation. Canned pack based on reports of Canners League of California and Northwest Canners Ass'n. Cases of 24 no. $2 \frac{1}{2}$ cans converted at 60 per ton except 62 in 1940 \& 1941. Barrelled pack: Calif. from Western Canner and Packer, Stat. Yearbook, April, 1940, p. 234 \& recent releases of Canners League; Northwest from Northwest Barrellers Ass' $n$. Converted at 8 barrels per ton. 


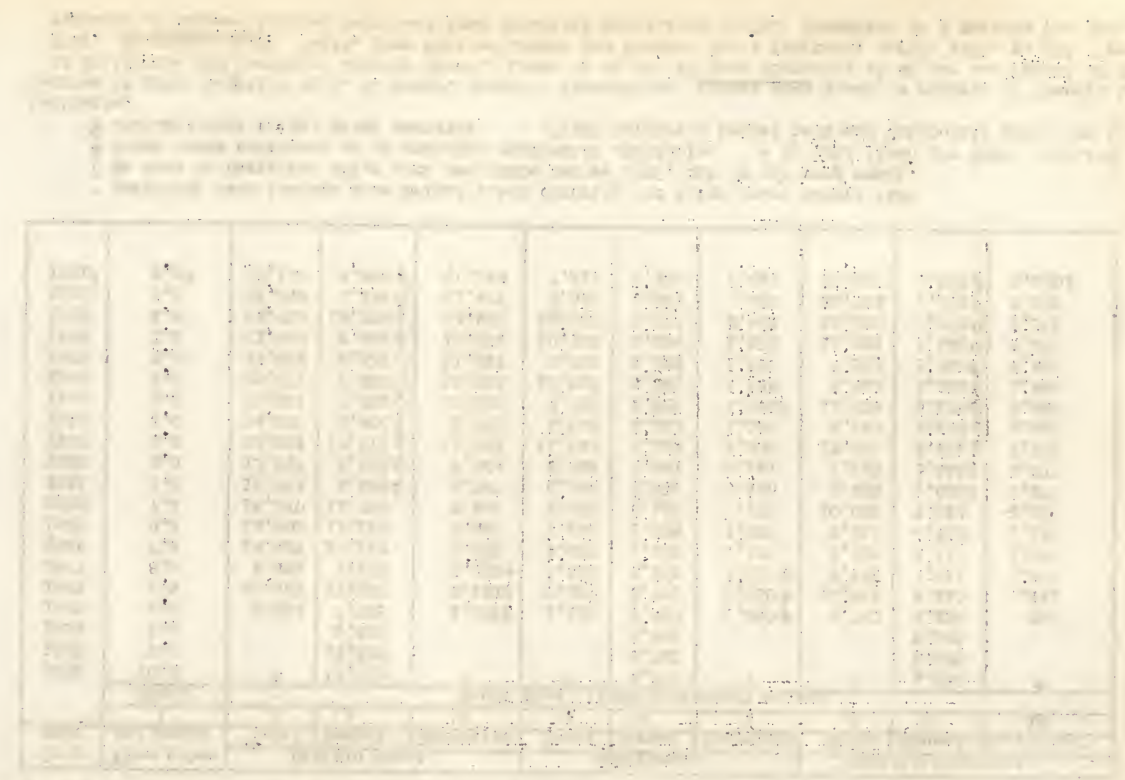

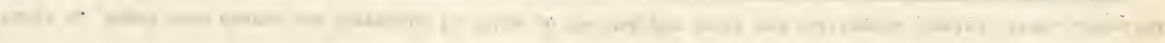


CHERTIFS

January, 1942

Table 5

Total Production* of Cherries in Far Western States, 1921-1941

\begin{tabular}{|c|c|c|c|c|c|c|c|c|}
\hline \multirow[b]{2}{*}{$\begin{array}{l}\text { Crop } \\
\text { year }\end{array}$} & \multirow{2}{*}{$\begin{array}{l}\text { States } \\
\text { listed, } \\
\text { total }\end{array}$} & \multirow[b]{2}{*}{$\begin{array}{c}\text { Pacific } \\
\text { Coast }\end{array}$} & \multirow[b]{2}{*}{ California } & \multicolumn{4}{|c|}{ Pacific Northwest } & \multirow[b]{2}{*}{ Utah } \\
\hline & & & & Total & Oregon & $\begin{array}{l}\text { Wash- } \\
\text { ington }\end{array}$ & Idaho & \\
\hline & \multicolumn{8}{|c|}{ Short tons } \\
\hline 1921 & 26,580 & 25,580 & 13,000 & 12,580 & 3,500 & 7,000 & 2.080 & 1,000 \\
\hline 1922 & 38,560 & 33,560 & 17,000 & 16,560 & 6,000 & 7,000 & 3,560 & 5,000 \\
\hline 1923 & 46,500 & 41,000 & 19,000 & 22,000 & 9,000 & 9,500 & 3,500 & 5,500 \\
\hline 1924 & 34,200 & 30,400 & 13,500 & 16,900 & 10,400 & 4,800 & 1,700 & 3,800 \\
\hline 1925 & 35,530 & 30,030 & 12,000 & 18,030 & 7,200 & 8,400 & 2,430 & 5,500 \\
\hline 1926 & 54,500 & 49,200 & 20,000 & 29,200 & 15,100 & 10,500 & 3,600 & 5,300 \\
\hline 1927 & 32,500 & 28,700 & 12,000 & 16,700 & 11,300 & 4,100 & 1,300 & 3,800 \\
\hline 1928 & 45,960 & 41,360 & 16,600 & 24,760 & 11,500 & 9,700 & 3,560 & 4,600 \\
\hline 1929 & $46,990 *$ & $43,790 *$ & 16,300 & $27,490 *$ & 9,000 & $15,500 *$ & 2,990 & 3,200 \\
\hline 1930 & $52,500 *$ & $48,800 *$ & 17,500 & $31,300 *$ & $12,600 *$ & $15,500 *$ & 3,200 & 3,700 \\
\hline 1931 & $47,300 *$ & $45,300 *$ & $23,000 *$ & $22,300 *$ & $9,000 *$ & $10,500 *$ & 2,800 & 2,000 \\
\hline 1932 & $55,780 *$ & $52,280 *$ & $18,500 *$ & $33,780 *$ & $14,000 *$ & $16,500 *$ & 3,280 & 3,500 \\
\hline 1933 & $64,940 *$ & $62,660 *$ & $25,300 *$ & 37,360 & $16,000 *$ & $18,500 *$ & 2,860 & 2,280 \\
\hline 1934 & $53,320 *$ & $50,920 \%$ & 17,000 & $33,920 *$ & $13,000 *$ & $18,000 *$ & 2,920 & 2,400 \\
\hline 1935 & $51,950 *$ & $49,750 *$ & 15,000 & $34,750 *$ & $15,800 *$ & $15,000 *$ & 2,950 & 2,200 \\
\hline 1936 & $61,890 *$ & 58,490 & 23,000 & $35,490 *$ & $15,600 *$ & $18,000 *$ & 1,890 & 3,400 \\
\hline 1937 & 52,600 & 50,500 & 21,500 & 28,900 & 13,800 & 13,500 & 1,600 & 2,100 \\
\hline 1938 & $84,530 *$ & $80,090 *$ & $30,000 \%$ & $50,090 *$ & $21,100 *$ & $26,500 *$ & $2,490 *$ & 4,440 \\
\hline 1939 & $88,250 *$ & $85,800 *$ & $36,000 *$ & $49,800 *$ & $21,200 *$ & $26,800 *$ & $1,800 \%$ & 2,450 \\
\hline 1940 & $69,450 *$ & $64,100 *$ & 11,000 & $53,100 *$ & $21,800 *$ & $29,100_{*} \neq$ & 2,200 & 5,350 \\
\hline $1941 \oint$ & 71,790 & 65,990 & 20,200 & 45,790 & 17,300 & 26,600 & 1,890 & 5,800 \\
\hline & & & & & & & & \\
\hline
\end{tabular}

* Data include unharvested tonnage for California estimated as: $1931,3,000$; $1932,2,500 ; 1933,400 ; 1938,4,800$; and $1939,3,000$. For urecon: 1930, 400; $1931,2,000 ; 1932,2,000 ; 1933,700 ; 1934,1,000 ; 1935,800 ; 1936,1,000 ; 1938$, 3,$600 ; 1939,2,000$; and 1940,270 . For Washington: $1929,2,000 ; 1930,1,500$;

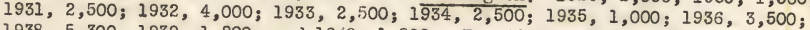
$1938,5,300 ; 1939,1,800$; and 1940, 1,800. For Idaho: 1938, 550, and 1939, 130. An average of about 85 per cent of the far westerm crop has been sweet cherries in recent years, and about 10 per cent of the crop in the rest of the United States (see table 6).

† Pacific Coast includes California, Oregon, Washington, and Idaho. cullage.

F Includes 700 tons of harvested sour cherries not utilized due to excessive

$\oint$ Prelininary estimates.

Sources of data:

Compiled by S. W. Shear, Giannini Foundation of Agricultural Fconomics, University of Califomia, from releases of the Thited States and California Crop Reporting Services. 


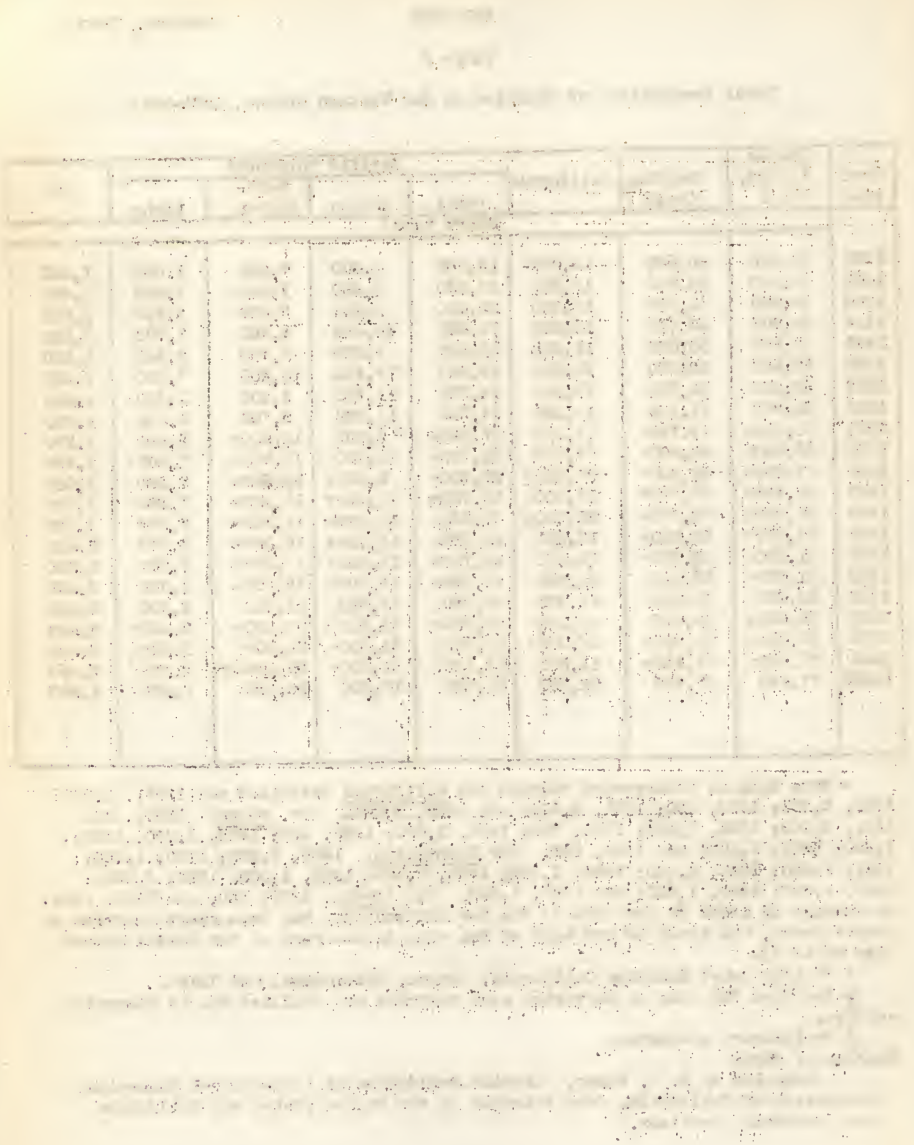


January 1942

\section{CHERRIES}

Table 6

United States Production* of Cherries by Chief States, Averages 1930-1941.

\begin{tabular}{|c|c|c|c|c|c|c|c|}
\hline \multirow[t]{2}{*}{ State } & \multirow{2}{*}{$\begin{array}{l}\text { Average } \\
1930-1933\end{array}$} & \multirow{2}{*}{$\begin{array}{l}\text { Average } \\
1934-1937\end{array}$} & \multicolumn{4}{|c|}{ Average 1938-1941 } & \multirow[b]{2}{*}{ Sourt } \\
\hline & & & Total & Sweet $t$ & Sourt & Sweet $t$ & \\
\hline \multirow[b]{3}{*}{ Total } & 1 & 2 & 3 & 4 & 5 & 6 & 7 \\
\hline & \multicolumn{5}{|c|}{ Short tons } & \multicolumn{2}{|c|}{ Per cent of total } \\
\hline & 131,340 & 132,280 & 167,250 & 76,090 & 91,160 & 45.5 & $54 \cdot 5$ \\
\hline Far Western & 55,130 & $54 ; 940$ & 78,500 & 07,480 & 11,020 & 86.0 & 14.0 \\
\hline California & 21,070 & 19,150 & 24,300 & 24,300 & 0 & 100.0 & 0.0 \\
\hline oregon & 12,900 & 14,550 & 20,350 & 18,240 & 2,110 & 89.6 & 10.4 \\
\hline Washington & 15,250 & 10,380 & 27,250 & 20,540 & 6,710 & 75.4 & 24.6 \\
\hline Idaho & 3,040 & 2,340 & 2,090 & 1,600 & 490 & 76.00 & 23.4 \\
\hline Utah & 2,870 & 2,520 & 4,510 & 2,800 & 1,710 & d2.1 & 37.9 \\
\hline Other states & 70,210 & 77,340 & 88,750 & 0,010 & 80,140 & 9.7 & 90.3 \\
\hline Colorado & 2,950 & 3,350 & 4,310 & 240 & 4,070 & 5.6 & 94.4 \\
\hline Nontana & 590 & 380 & 380 & 70 & 310 & $18 \cdot 4$ & 81.6 \\
\hline New York & 20,200 & 19,640 & 20,820 & 1,840 & 18,980 & 8.8 & 91.2 \\
\hline Pennsylvania & 7,930 & 8,150 & 10,560 & 3,020 & 7,540 & 28.6 & 71.4 \\
\hline Ohio & 4,740 & 5,540 & 7,190 & 370 & 6,820 & $5 \cdot 1$ & 94.9 \\
\hline Michigan & 30,780 & 31,560 & 33,910 & 3,070 & 30,840 & 9.1 & 90.9 \\
\hline Wisconsin & 8,990 & 8,720 & 11,560 & 0 & 11,580 & 0.0 & 100.0 \\
\hline
\end{tabular}

* Includes harvested and unharvested production.

+ Official estimates of production of sweet and sour varieties separately have not been published for years prior to 1938.

Source of data: Compiled by S. W. Shear, Giannini Foundation of Agricultural Economios, University of California, from releases of the United States Crop Reporting Service. Cols. 6 and 7 oalculated from data in cols. 3,4 , and 5 . 

University of California, College of Agriculture

Agricultural Experiment Station, Berkeley, January, 1942

\section{DECIDUOUS FRUIT STATISTICS}

\section{DATES}

Table 1

California Dates: Bearing Acreage, Production, Yield and Farm Value, 1924-1941

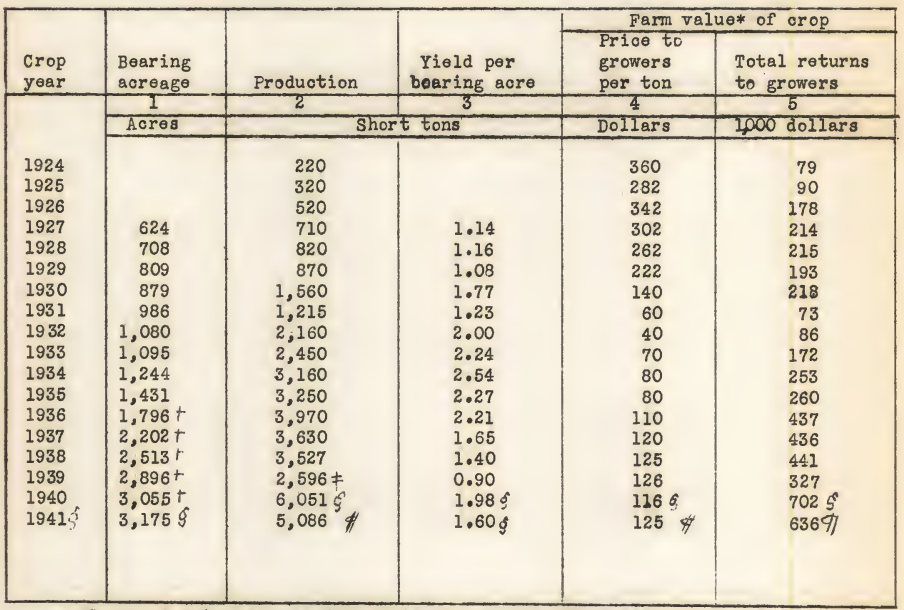

*Growers' returns for naked fruit at growers' flrst delivery point.

$1940,325$.

†Nonbearing acres: 1936, 1,500; 1937, 1,046; 1938, 766; 1939, 469; and

+About half of the prospective 1939 California date orop is excluded from

1939 data because of severe damage from rains in September 1939.

Greliminary.

4f Riverside County production and price only for 1941. It produced about

94 per cent of the 1940 California crop.

Source of data: Compiled by S. W. Shear, Giannini Foundation of Agricultural

Economics, University of California, from data obtained from the California

Crop Reporting Service. Production as given is the delivered weight at the packing house. 


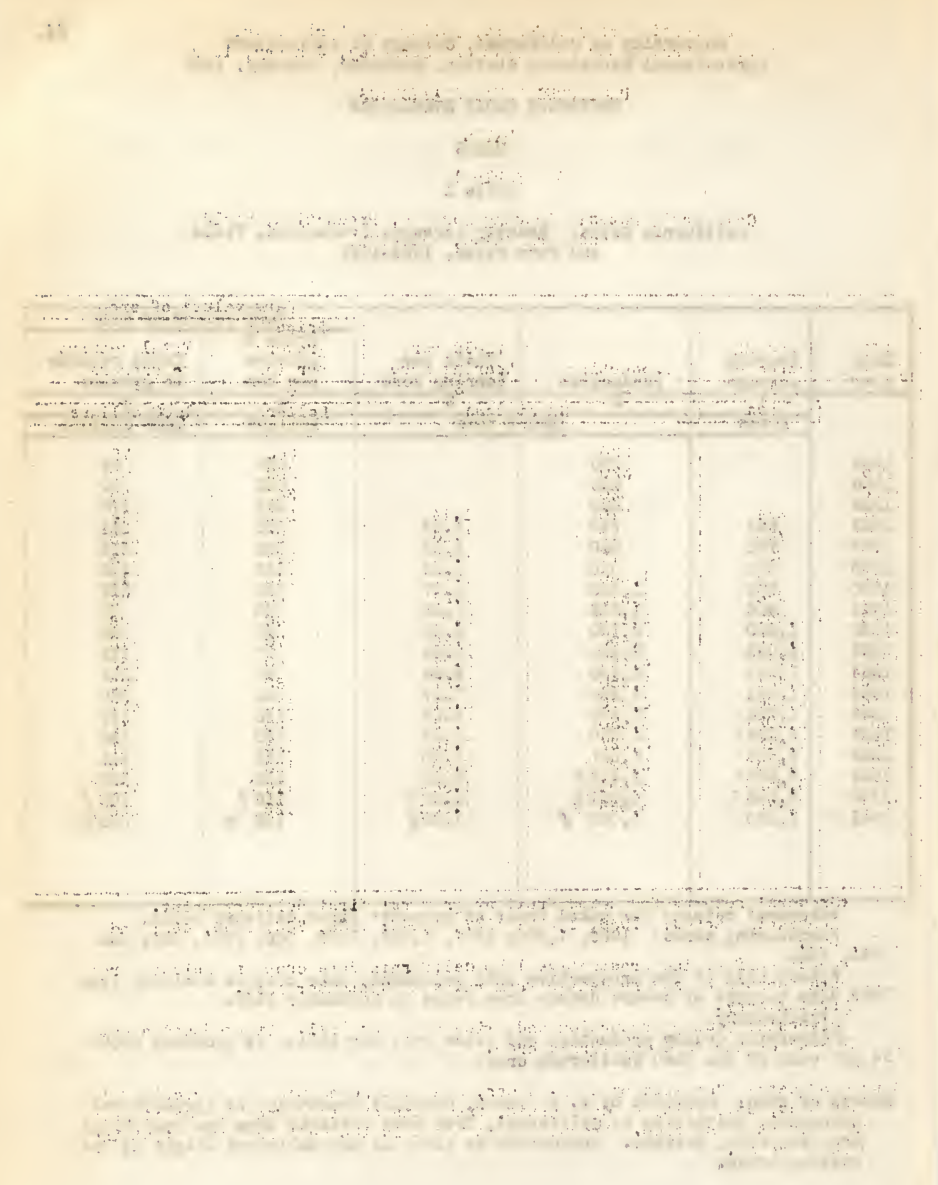


Table 2. United States Production, let Imports and Apparent Per Capita Consumption of Dates, 1915-1941

\begin{tabular}{|c|c|c|c|c|c|c|}
\hline \multirow{2}{*}{\begin{tabular}{|c|} 
Year \\
beginning \\
July 1
\end{tabular}} & \multicolumn{3}{|c|}{ United States production } & \multirow[b]{2}{*}{ Net importst } & \multirow{2}{*}{$\begin{array}{c}\text { Available } \\
\text { for } \\
\text { consumption }\end{array}$} & \multirow{2}{*}{$\begin{array}{l}\text { Apparent } \\
\text { per-capita } \\
\text { consumption }\end{array}$} \\
\hline & Califomia* & Arizona & Total & & & \\
\hline \multirow[b]{3}{*}{ Averages: } & 1 & 2 & 3 & $\overline{4}$ & 5 & 6 \\
\hline & \multicolumn{5}{|c|}{ Thousands of pounds $\neq$} & Pounds \\
\hline & & & & & & \\
\hline $1915-1919$ & 57 & 20 & 77 & 22,733 & 22,810 & 0.22 \\
\hline $1920-1924$ & 236 & 14 & 250 & 45,472 & 45,722 & 0.41 \\
\hline $1925-1929$ & 1,296 & 40 & 1,336 & 50,927 & 52,263 & 0.44 \\
\hline $1930-1934$ & 4,218 & 143 & 4,361 & 46,983 & 51,344 & 0.41 \\
\hline $1935-1939$ & 6,789 & 255 & 7,044 & 51,176 & 58,220 & 0.45 \\
\hline \multicolumn{7}{|l|}{ Annual: } \\
\hline 1920 & 120 & 0 & 120 & 33,824 & 33,944 & 0.32 \\
\hline 1921 & 122 & 2 & 124 & 44,512 & 44,636 & 0.41 \\
\hline 1922 & 210 & 16 & 226 & 50,078 & 50,304 & 0.45 \\
\hline 1923 & 290 & 24 & 314 & 39,069 & 39,383 & 0.35 \\
\hline 1924 & 440 & 26 & 466 & 59,877 & 60,343 & 0.53 \\
\hline 1925 & 640 & 29 & 669 & 66,192 & 66,851 & 0.57 \\
\hline 1926 & 1,040 & 35 & 1,075 & 45,500 & 46,575 & 0.39 \\
\hline 1927 & 1,420 & 39 & 1,459 & 40,725 & 42,184 & 0.35 \\
\hline 1928 & 1,640 & 47 & 1,687 & 51,451 & 53,138 & 0.44 \\
\hline 1929 & 1,740 & 50 & 1,790 & 50,767 & 52,557 & 0.43 \\
\hline 1930 & 3,120 & 50 & 3,170 & 41,255 & 44,426 & 0.36 \\
\hline 1931 & 2,430 & 120 & 2,550 & 43,452 & 46,002 & 0.37 \\
\hline 1932 & 4,320 & 170 & 4,490 & 46,437 & 50,927 & 0.41 \\
\hline 1933 & 4,900 & 200 & 5,100 & 49,988 & 55,088 & 0.44 \\
\hline 1934 & 6,320 & 175 & 6,495 & 53,781 & 60,276 & 0.47 \\
\hline 1935 & 6,500 & 200 & 6,700 & 54,057 & 60,757 & 0.47 \\
\hline 1936 & 7,940 & $250 \oint$ & $8,190 \oint$ & 58,137 & 66,327 \& & $0.51 \$$ \\
\hline 1937 & 7,260 & $450 \oint$ & 7,7109 & 51,643 & 59,353 & $0.46 \oint$ \\
\hline 1938 & 7,054 & 225 \% 9 & 7,2799 & 46,948 & 54,2278 & $0.41 \delta$ \\
\hline 1939 & 5,1929 & $150 \% \mathrm{~g}$ & 5,3429 & 45,093 & $50,435 \varnothing$ & $0.38 \%$ \\
\hline 1940 & 12,1026 & $350 \oint$ & $12,452 \%$ & 44,789 & $57,241 \oint$ & 0.438 \\
\hline 1941 & $10,172 * 6$ & $100 \oint$ & $10,272 \oint$ & & & \\
\hline
\end{tabular}

*Riverside County only 1915-1918 and 1941, but it produced alout 95 per cent of the California crop in recent years.

+ Net imports prior to 1933 are total imports minus re-exports, but beginning 1933 are imports for consumption only.

F Data on production are delivered weight at packing house, and imports are declared import weight, mostly dried -- some pitted and some unpitted.

$\oint$ Preliminary estimates.

4 Arizona data as given for 1938 and 1939 are harvested production only; total production was about 250,000 pounds in 1938 and about 500,000 pounds in 1939. About half of the prospective 1939 California date cron is excluded from 1939 data because of severe damage from rains in Septenber 1939.

Sources of data: Compiled by S. . Shear, Giannini poundation. Col. 1: 19151918 and 1941 from annual mimeographed crop reports of Biverside County Agricultural Cormissioner. 1919-1940 from reports of the Califormia Cron Reporting Service. Col. 2: Estimates by comespondence from Dept. Horticulture, Arizona Agr. Dixp. Station. Cols 3 and 5: Obtained by addition. Col. 4: From official reports of the U. S. Jur. For. and Dom. Com. Col. 6: Col. 5 divided by United States population figures of January 1. 
$+\quad \therefore$

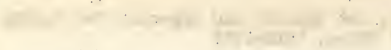

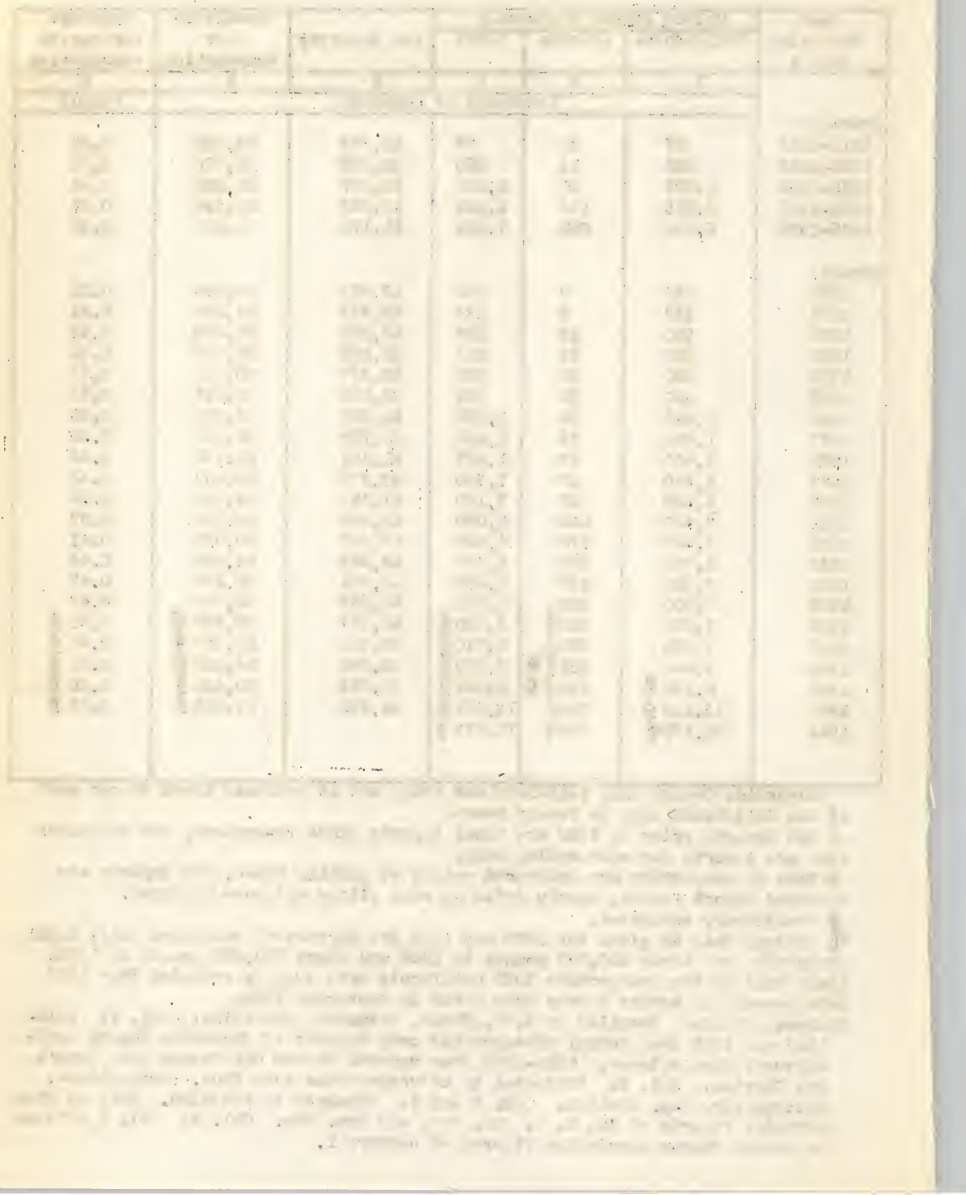


DATES

January, 1942

56.

Table 3. United States Imports of Dates by Countries of origin, 1925-1941

\begin{tabular}{|c|c|c|c|c|c|c|c|}
\hline $\begin{array}{c}\text { Calendar } \\
\text { year }\end{array}$ & Total & Iraq & $\begin{array}{l}\text { United } \\
\text { Kingdom }\end{array}$ & Arabia & Belgium & France & others \\
\hline \multirow{2}{*}{\multicolumn{8}{|c|}{ Thousands of pounds* }} \\
\hline & & & & $t$ & & & \\
\hline $\begin{array}{l}1925 \\
1926\end{array}$ & $\begin{array}{l}78,706 \\
49,280\end{array}$ & $\begin{array}{l}60,866+ \\
42,830+\end{array}$ & $\begin{array}{r}13,290 \\
3,348\end{array}$ & $\begin{array}{l}t \\
t\end{array}$ & $\begin{array}{r}0 \\
86\end{array}$ & $\begin{array}{l}372 \\
658\end{array}$ & $\begin{array}{l}4,178 \\
2,358\end{array}$ \\
\hline 1927 & 38,408 & 32,122 & 3,964 & 560 & 24 & 536 & 1,202 \\
\hline 1928 & 58,842 & 50,412 & 5,454 & 880 & 764 & 580 & 752 \\
\hline 1929 & 54,134 & 48,318 & 2,372 & 812 & 1,812 & 402 & 418 \\
\hline 1930 & 41,854 & 35,485 & 4,723 & 943 & 101 & 314 & 288 \\
\hline 1931 & 44,039 & 33,135 & 9,130 & 194 & 192 & 12 & 1,376 \\
\hline 1932 & 44,967 & 31,171 & 12,913 & 266 & 273 & 31 & 313 \\
\hline 1933 & 47,493 & 30,144 & 14,593 & 872 & 1,528 & 0 & 356 \\
\hline $1934 \neq$ & 48,835 & 40,462 & 3,989 & 1,815 & 1,263 & 0 & 1,306 \\
\hline $1935 *$ & 60,218 & 47,130 & 10,275 & 1,764 & 598 & 1 & 450 \\
\hline $1936 \neq$ & 53,299 & 44,891 & 2,955 & 4,422 & 205 & $-\hat{q}$ & 826 \\
\hline $1937 \neq$ & 55,972 & 51,291 & 484 & 2,394 & 7 & 1 & 1,795 \\
\hline $1938 \neq$ & 45,482 & 43,923 & 287 & 700 & 0 & 1 & 571 \\
\hline $1939 \neq$ & 43,695 & 41,331 & 57 & 25 & 4 & 0 & 2,278 \\
\hline $1940 \%$ & 44,238 & 40,847 & 0 & 0 & 0 & - & 3,391 \\
\hline \multicolumn{8}{|l|}{ Jan-Sөpt: $\not f$} \\
\hline 1940 & 18,240 & 17,697 & 0 & 0. & 0 & - & 543 \\
\hline 1941 & 19,142 & 18,136 & 0 & 0 & 0 & 0 & 1,006 \\
\hline \multicolumn{8}{|l|}{ Averages : } \\
\hline $1925-2929$ & 55,874 & 46,910 & 5,686 & 450 & 537 & 510 & 1,781 \\
\hline $1930-1934$ & 45,438 & 34,079 & 9,070 & 818 & 671 & 72 & 728 \\
\hline $1935-1939$ & 51,733 & 45,713 & 2,811 & 1,861 & 163 & 1 & 1,184 \\
\hline \multirow{4}{*}{$\begin{array}{c}\text { Averages : } \\
1925-1929 \\
1930-1934 \\
1935-1939\end{array}$} & \multicolumn{7}{|c|}{ Per cent of total } \\
\hline & & 83.9 & 10.2 & & & & \\
\hline & 100.0 & 75.0 & $\begin{array}{l}10.2 \\
20.0\end{array}$ & $\begin{array}{l}0.8 \\
1.8\end{array}$ & $\begin{array}{l}1.0 \\
1.5\end{array}$ & $\begin{array}{l}0.9 \\
0.1\end{array}$ & $\begin{array}{l}3.2 \\
1.6\end{array}$ \\
\hline & 100.0 & 88.4 & 5.4 & 3.6 & 0.3 & $-\infty$ & 2.3 \\
\hline
\end{tabular}

*Declared import weight, mostly dried -- some pitted and some unpitted.

tData for 1925 of $60,866,000$ pounds reported in original source as

imported from Arabia (inoluding Iraq and Mesopotamia) and for 1926 of

$42,830,000$ pounds reported as $32,430,000$ pounds from Arabia and $10,400,000$

pounds from Iraq, all assumed as from Iraq.

† Beginning with 1934 data are imports for consumption only.

$\S$ Dashes indicate less than 500 pounds or less than 0.05 per cent.

\# Import data are not available after September, 1941.

Sources of data: Compiled by S.W. Shear, Giannini Foundation.

1925-1938: Dept. Com., Foreign Commerce and Navigation of U. S.

1939-1941: U. S. Dept. Com. monthly mimeographed statements No. 3052. 


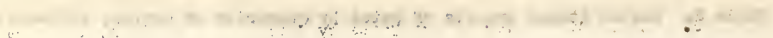

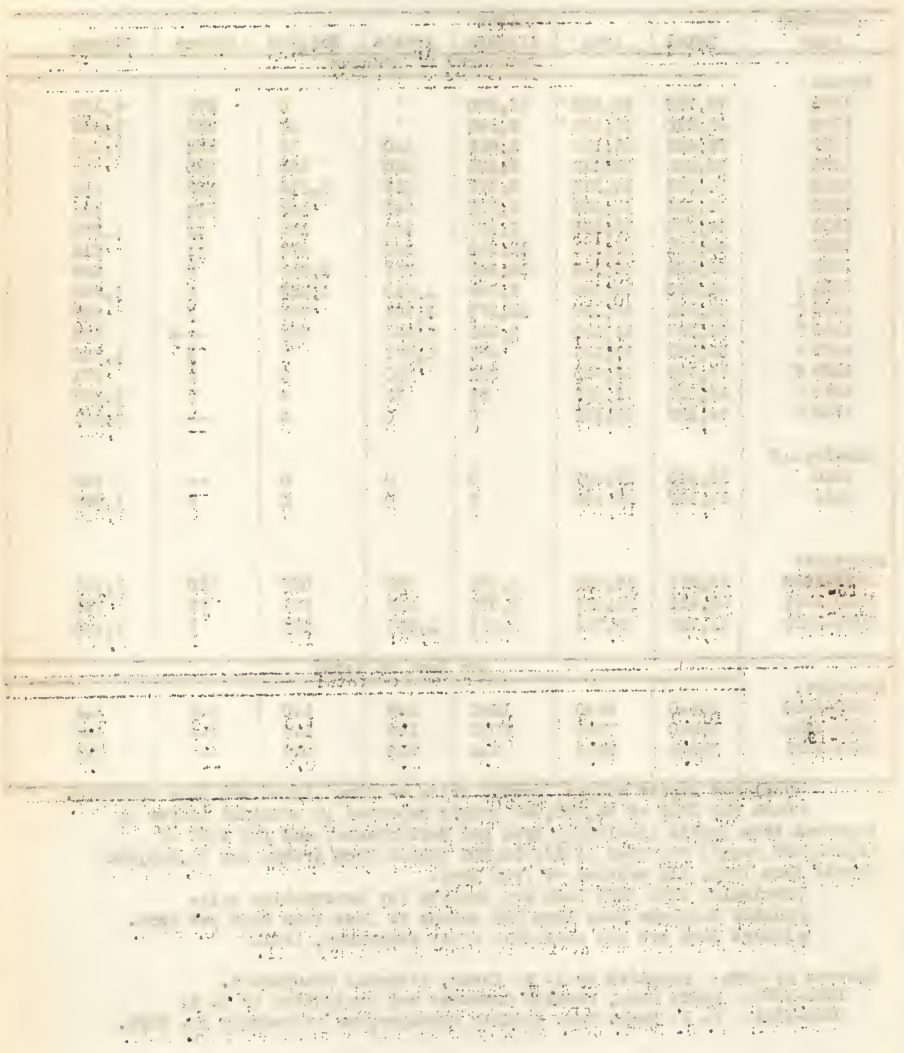


University of California, college of hgriculture

Agricultural Experiment Station, Berkeley, January 1942

DECIDUOUS FRUIT STATISTICS

FIGS

Table 1. Califorria Figs*: Bearing Acreage, Production, Condition, Yield, and Farm Value, 1919-1941

\begin{tabular}{|c|c|c|c|c|c|c|}
\hline \multirow[b]{2}{*}{$\begin{array}{l}\text { Crop } \\
\text { year }\end{array}$} & \multirow[b]{2}{*}{$\begin{array}{l}\text { Bearing } \\
\text { acreage }\end{array}$} & \multicolumn{2}{|c|}{ Production } & \multirow[b]{2}{*}{$\begin{array}{l}\text { Yield per } \\
\text { bearing } \\
\text { acre }\end{array}$} & \multicolumn{2}{|c|}{ Farm tvalue of crop } \\
\hline & & Total & $\begin{array}{l}\text { Nov } 1 \\
\text { percent of } \\
\text { full crop }\end{array}$ & & $\begin{array}{l}\text { Price to } \\
\text { growers } \\
\text { per dry ton }\end{array}$ & $\begin{array}{c}\text { Total } \\
\text { returns } \\
\text { to } \text { growers } \\
\end{array}$ \\
\hline & 1 & 2 & 3 & 4 & 5 & 6 \\
\hline & Acres & $\begin{array}{l}\text { Short tons, } \\
\text { dry weight }\end{array}$ & Per cent & $\begin{array}{l}\text { Short tons, } \\
\text { dry weight }\end{array}$ & Dollars & $\begin{array}{c}1,000 \\
\text { dollars }\end{array}$ \\
\hline 1919 & 10,500 & 12,300 & 108 & 1.2 & $156 \neq$ & $1,920 \neq$ \\
\hline 1920 & 11,023 & 12,700 & 94 & 1.2 & $97 \neq$ & $1,227 \neq$ \\
\hline 1921 & 11,472 & 10,133 & 82 & 0.9 & $158 \neq$ & $1,605 \neq$ \\
\hline 1922 & 12,774 & 11,867 & 90 & 0.9 & $133 \neq$ & 1,58 क \\
\hline 1923 & 16,979 & 10,600 & 83 & 0.6 & $112 \neq$ & $1,185 \neq$ \\
\hline 1924 & 21,328 & 9,233 & 60 & 0.4 & 117 & 1,079 \\
\hline 1925 & 23,440 & 10,633 & 78 & 0.5 & 128 & 1,366 \\
\hline 1926 & 29,537 & 13,100 & 81 & 0.4 & 131 & 1,711 \\
\hline 1927 & 42,595 & 13,800 & 80 & 0.3 & 78 & 1,080 \\
\hline 1928 & 47,038 & 13,533 & 60 & 0.3 & 78 & 1,049 \\
\hline 1929 & 46,353 & 19,433 & 79 & 0.4 & 116 & 2,260 \\
\hline 1930 & 46,728 & 23,567 & 86 & 0.5 & 72 & 1,701 \\
\hline 1931 & 46,142 & 19,100 & 58 & 0.4 & 57 & 1,095 \\
\hline 1932 & 45,760 & 21,167 & 76 & 0.5 & 34 & 721 \\
\hline 1933 & 42,744 & 23,467 & 68 & 3.5 & 53 & 1,240 \\
\hline 1934 & 41,526 & 26,500 & 68 & 0.6 & 70 & 1,861 \\
\hline 1935 & 38,000 & 27,400 & 77 & 0.7 & 59 & 1,608 \\
\hline 1936 & 38,293 & 23,667 & 69 & 0.6 & 90 & 2,134 \\
\hline 1937 & 38,206 & 32,700 & 77 & 0.9 & 86 & 2,796 \\
\hline 1938 & $37,689 \oint$ & 35,167 & 78 & 0.9 & 76 & 2,673 \\
\hline 1939 & 37,0556 & 29,100 & 72 & 0.8 & 85 & 2,485 \\
\hline 1940 & $35,533^{\circ}$ & 37,000 & 82 & 1.0 & 84 & 3,102 \\
\hline 19414 & 35,5004 & $39,133 \neq$ & 75 & 1.1 & 124 & $4,833 \neq$ \\
\hline
\end{tabular}

* Total production of all figs includes dried (merchantable and nonmercharitable), canning, and fresh figs. Coverted to dry woight at 3 to 1 drying ratio.

+ Growers' returns for naiced fruit at growers' first delivery point.

F Partly preliminary unofficial estimates.

Nonbear ing acres: 1938, 1,110; 1939, 1,001; and 1940, 764.

की Preliminary.

Sources of data: Compiled by S. w. Shear, Giannini Foundation of Agricultural Economics, University of california, from reports of the United States and California Crop keportins Services and Dried Fruit hssociation of California, except col. 6 in 1919-1923 involves estimates by S. ". Shear for farm value of fresh and canned figs; col. 5 is col. 6 divided by col.2 
$\therefore$

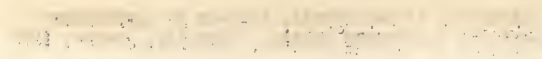

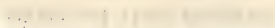

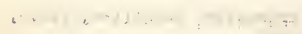

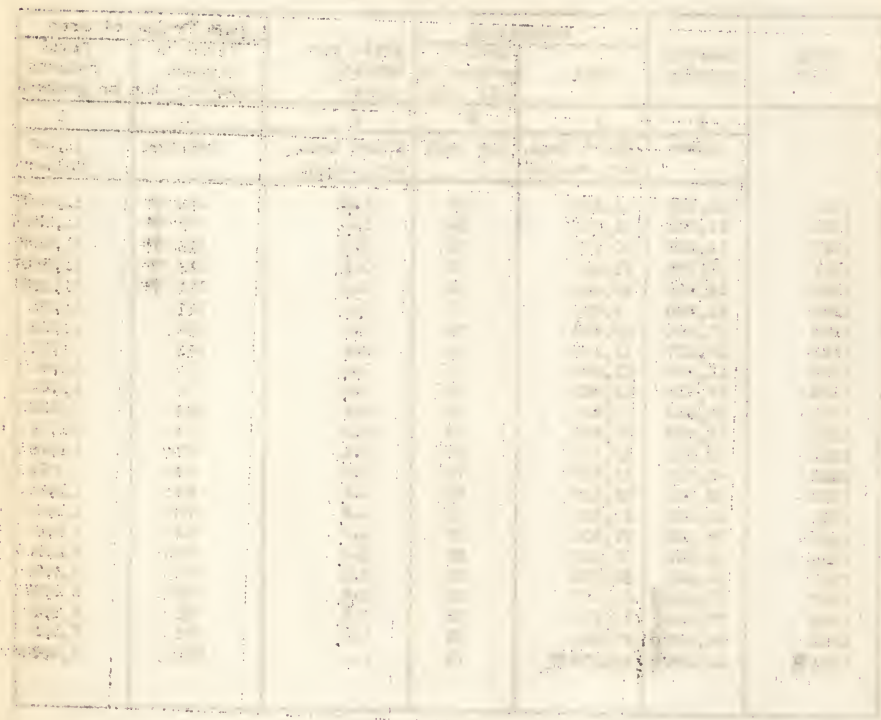

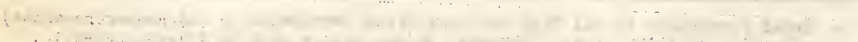

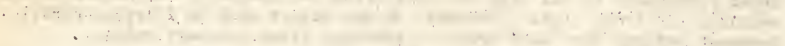

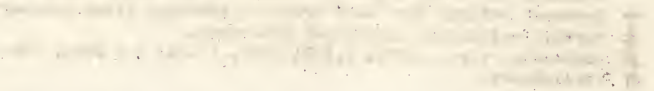

$\therefore \ldots+1 \cdot$

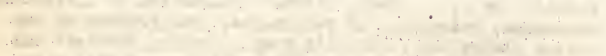

. 
FIGS

Table 2

California Figs: Produotion* and Utilization, 1921-1941

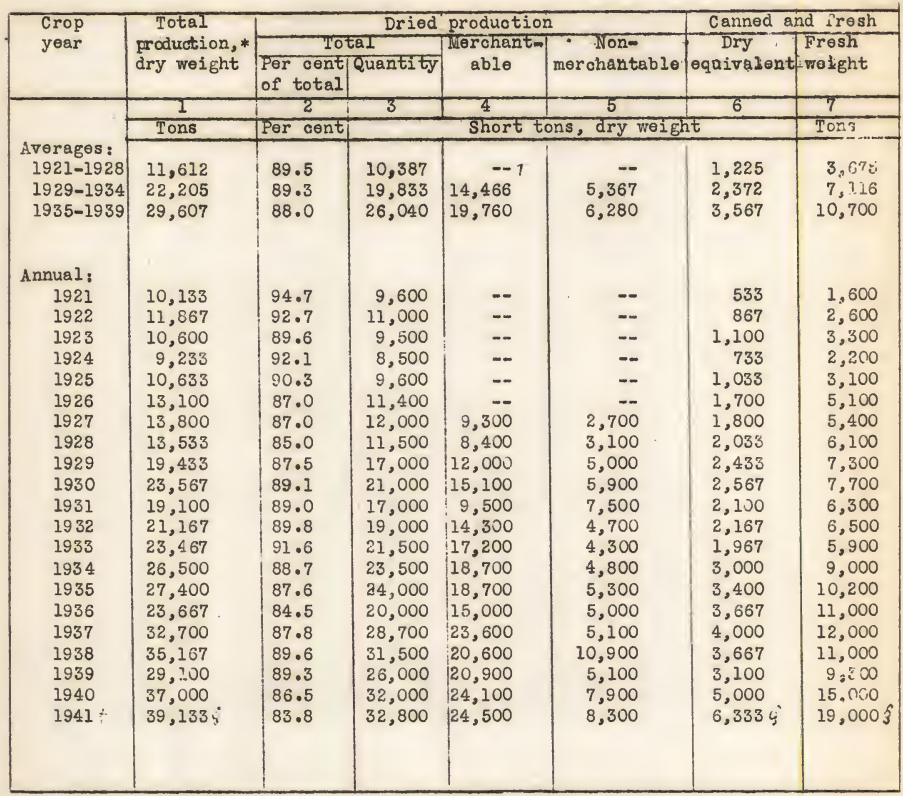

*Includes merohantable and nonmerohantable dried figs.

+Dashes indicate data not available; the nunmerchantable tonnage is believed to have been very small prilor to 1927 .

fPreliminary data.

bUnofficial estimates.

Sources of data: Compiled by S.W. Shear, Giannini Foundation of Agricultural Economios, University of california.

Cols. 1,2 and 6: Computed from other data' in this table.

Cols. $3 ; 4,5$ and 7: Estimates of the U.S, and California Crop Reporting

Services and of the Dried Fruit Association of California. 


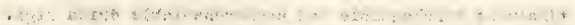

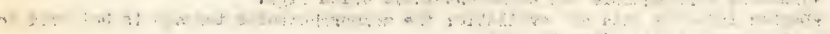

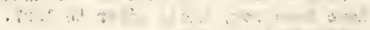

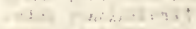

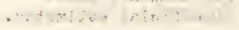

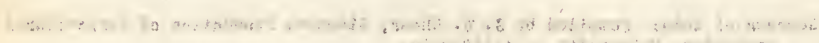

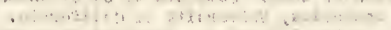
-

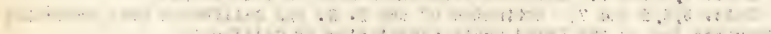

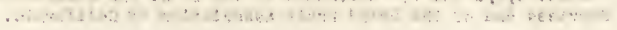


FIGS

Table 3. California and Texas Figs Marketed Fresh and Canned, 1922-1941

\begin{tabular}{|c|c|c|c|c|c|c|c|c|c|}
\hline \multirow{3}{*}{$\begin{array}{l}\text { Crep } \\
\text { year }\end{array}$} & \multicolumn{7}{|c|}{ Quantity } & \multicolumn{2}{|c|}{ Farm price } \\
\hline & \multirow{2}{*}{\multicolumn{6}{|c|}{$\frac{\text { California }}{\text { Used fresh }}$}} & \multirow{2}{*}{$\begin{array}{l}\text { Texas } \\
\text { Canned }\end{array}$} & \multirow{2}{*}{$\begin{array}{l}\text { California* } \\
\text { Per ton }\end{array}$} & \multirow{2}{*}{$\begin{array}{l}\text { Texas } \\
\text { Per ton }\end{array}$} \\
\hline & \multirow{2}{*}{ Total } & \multirow{2}{*}{ Canned } & & & \multicolumn{2}{|c|}{$\begin{array}{l}\text { Used fresh } \\
\text { In state } \\
\text { Interstate shipments }\end{array}$} & & & \\
\hline & & & $\frac{\text { Total }}{3}$ & $\frac{\text { In state }}{4}$ & $\frac{\text { Interstate }}{5}$ & $\frac{\text { shipments } t}{6}$ & 7 & 8 & 9 \\
\hline & \multicolumn{5}{|c|}{ Short tons, fresh weight } & Cars & Short tons & \multicolumn{2}{|c|}{ Dollars } \\
\hline 1922 & 2,600 & 1,700 & 900 & 800 & 100 & 10 & $-\neq$ & $-\infty$ & - \\
\hline 1923 & 3,300 & 2,200 & 1,100 & 900 & 200 & 20 & .909 & - & - \\
\hline 1924 & 2,200 & 900 & 1,300 & 1,100 & 200 & 21 & 1,180 & 104.00 & 104.00 \\
\hline 1925 & 3,100 & 1,400 & 1,700 & 1,300 & 400 & 43 & 2,240 & 100.00 & 76.00 \\
\hline 1926 & 5,100 & 3,000 & 2,100 & 1,300 & 800 & 85 & 4,978 & 112.00 & 76.00 \\
\hline 1927 & 5,400 & 3,000 & 2,400 & 1,300 & 1,100 & 116 & 4,879 & 100.00 & 59.00 \\
\hline 1928 & 6,100 & 4,000 & 2,100 & 1,200 & 900 & 98 & 6,513 & 87.00 & 65.00 \\
\hline 1929 & 7,300 & 4,100 & 3,200 & 2,000 & 1,200 & 134 & 2,778 & 100.00 & 40.00 \\
\hline 1930 & 7,700 & 4,300 & 3,400 & 2,400 & 1,000 & 110 & 2,961 & 90.00 & 45.00 \\
\hline 1931 & 6,300 & 1,400 & 4,900 & 4,300 & 600 & 68 & 1,851 & 74.00 & 59.00 \\
\hline 1932 & 6,500 & $2,600\}$ & 3,900 & 3,300 & 600 & 68 & 510 & 36.50 & 47.00 \\
\hline 1933 & 5,900 & 2,300 & 3,600 & 3,300 & 300 & 36 & 655 & 50.50 & 55.00 \\
\hline 1934 & 9,000 & 3,9009 & 5,100 & 4,600 & 500 & 58 & 966 & 51.85 & 52.00 \\
\hline 1935 & 10,200 & $3,800 \xi$ & 6,400 & 5,800 & 600 & 68 & 1,590 & 56.50 & 64.00 \\
\hline 1936 & 11,000 & $5,600 \mathrm{~s}$ & 5,400 & 4,800 & 600 & 67 & 1,450 & 54.00 & 59.00 \\
\hline 1937 & 12,000 & 7,400 & 4,600 & 3,800 & 800 & 88 & 1,610 & 70.30 & 60.00 \\
\hline 1938 & 11,000 & 4,800 & 6,200 & 5,600 & 600 & 72 & 1,240 & 56.50 & 45.00 \\
\hline 1939 & 9,300 & 4,000 & 5,300 & 4,600 & 700 & 82 & 1,140 & 49.10 & 56.00 \\
\hline 1940 & 15,000 & 7,400 & 7,600 & 6,400 & 1,200 & 130 & 840 & 57.50 & 63.00 \\
\hline 19414 & 19,$000 ; 1$ & 11,700 & $7,300 \|$ & 6,40011 & 900 & 86 & 1,400 & 57.60 & 64.00 \\
\hline & & & & & & & & & \\
\hline
\end{tabular}

- Returns to growers for naked fruit delivered at the growers' first delivery point.

in glass. Fresh shipments only. \# \# Preliminary data subject to revision. Sources of data: $\quad$ // Unofficial estimates.

Cols. 1, 7, 8, and 9: From U. S. and Cannini Foundation of Agricultural Economics, University of California.

Co1. 2: 1922-1926 estimates by and California Crop Reports except 1923 Texas data are unofficial estimates.

estimates of glass pack in 1937-1941.

Cols. 3 and $4:$ Computed from other data given in this table.

Co1: 5: Çmputed from col 12 to nearest hundred tons at 9 tons per car, except 1941 at $10 \frac{1}{2}$. 


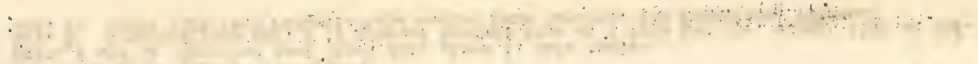

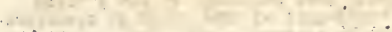

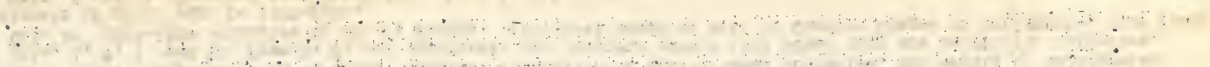

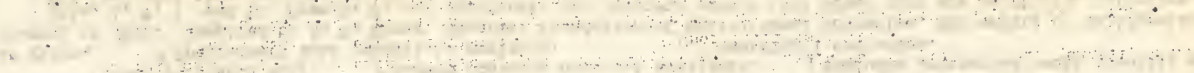

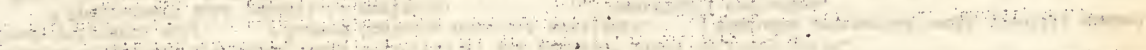

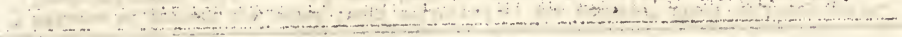

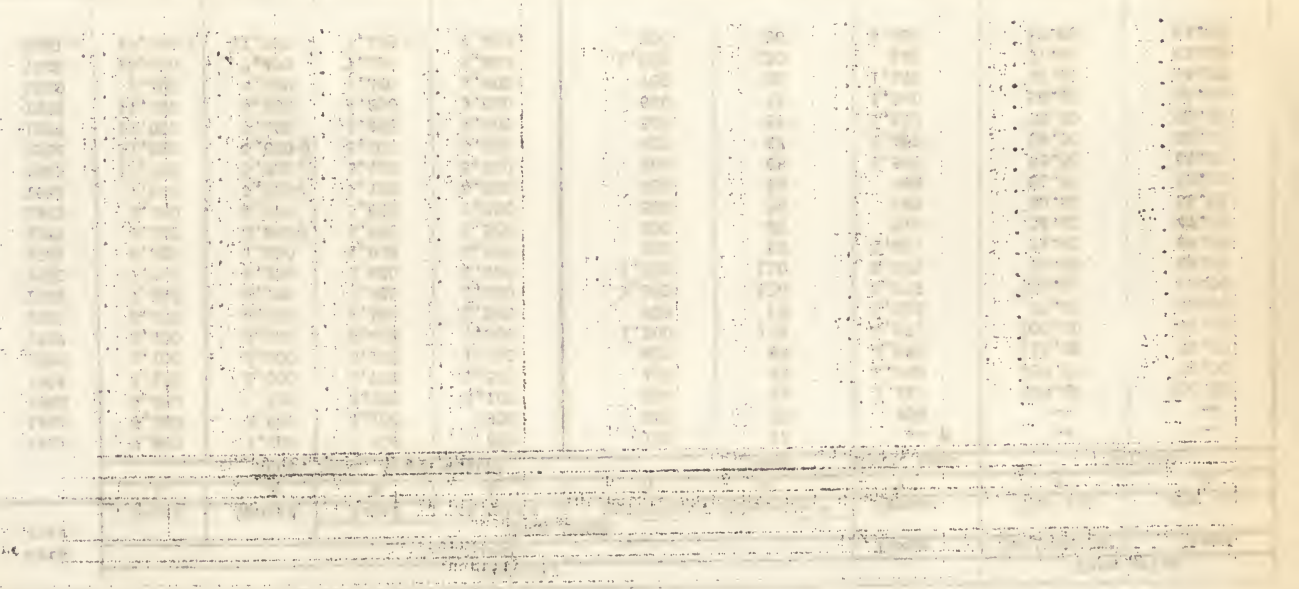


Table 4

California Dried Figs: Farm and F.O.B. Prices, 1921-1941

\begin{tabular}{|c|c|c|c|c|c|}
\hline \multirow[b]{2}{*}{$\begin{array}{l}\text { Crop } \\
\text { year }\end{array}$} & \multirow[b]{2}{*}{$\begin{array}{l}\text { Farm } \\
\text { price* }\end{array}$} & \multicolumn{4}{|c|}{ F.0.b. California packers' quotations } \\
\hline & & $\begin{array}{l}\text { Choice } \\
\text { Black }\end{array}$ & $\begin{array}{l}\text { Choice } \\
\text { Calimyrna }\end{array}$ & $\begin{array}{c}\text { Choice } \\
\text { Adriatio } \\
\end{array}$ & $\begin{array}{c}\text { Adriatic } \\
\text { paste in } \\
80^{\prime} \mathrm{s}\end{array}$ \\
\hline & $I$ & 2 & 3 & 4 & 5 \\
\hline & \multicolumn{5}{|c|}{ Cents per pound } \\
\hline 1921 & 7.2 & 8.2 & $10.5+$ & 8.8 & \\
\hline 1922 & 6.0 & 13.3 & 14.8 & 9.1 & \\
\hline 1923 & 4.5 & 7.8 & 8.5 & 5.8 & \\
\hline 1924 & 5.0 & $9 \cdot 3$ & 8.2 & 6.1 & \\
\hline 1925 & 5.5 & $12 \cdot 8$ & $7.5+$ & 5.7 & \\
\hline 1926 & 5.0 & 9.1 & 7.3 & 6.2 & \\
\hline 1927 & 2.2 & 6.0 & $-\ldots$ & 5.0 & \\
\hline 1928 & 2.2 & 7.1 & 9.5 & 6.2 & \\
\hline 1929 & 4.5 & 8.3 & 10.0 & 9.4 & \\
\hline 1930 & 2.4 & 4.2 & 7.8 & 5.9 & 4.8 \\
\hline 1931 & 1.8 & 4.1 & 6.0 & 5.5 & 4.5 \\
\hline 1932 & 1.3 & 3.3 & 4.7 & 3.7 & 3.2 \\
\hline 1933 & 2.2 & 5.6 & 6.5 & 5.7 & 4.7 \\
\hline 1934 & 3.0 & 5.8 & 6.5 & 7.0 & 6.0 \\
\hline 1935 & 2.2 & 4.8 & 7.0 & 6.3 & 5.5 \\
\hline 1936 & 3.9 & 5.1 & 8.25 & 8.0 & 6.4 \\
\hline 1937 & 3.4 & 4.7 & 7.4 & 6.6 & 5.5 \\
\hline 1938 & 3.3 & 3.7 & 6.9 & 6.1 & 5.1 \\
\hline 1939 & 3.9 & 5.2 & 8.1 & 7.7 & 6.6 \\
\hline 1940 & 3.5 & 4.5 & 8.1 & 7.7 & 6.2 \\
\hline 1941 & $5.7 \pi$ & 6.4 & $15 \cdot 6$ & $12 \cdot 6$ & 10.4 \\
\hline & & 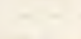 & & & \\
\hline & & & & & \\
\hline
\end{tabular}

*Growers' returns for naked fruit delivered at growers' first delivery point; prices are for produotion of all dried figs, as given in table 2, col. 3 . + Partially estimated by S. W. Shear.

Fashes indicate no quotations reported.

squtations for two weeks only. \# Preliminary.

Sources of data: Compiled by S.W. Shear, Giannini Foundation of Agricultural Economics, University of california.

Col. 1: From Reports of the California Cooperative crop Reporting Service. Cols. 2-5: Simple averages of monthly f.o.b. quotations from opening of new crop through December 31. Based upon California Fruit News, weekly quotations on 50-pound boxes and 25-pound boxes in recent years. 1921-1929, monthly prices are simple averages of weekly quotations and the weekly data were averages of the range. 1930-1941, monthly prices are simple averages of the lower of the range of weekly quotations. 


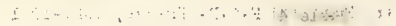
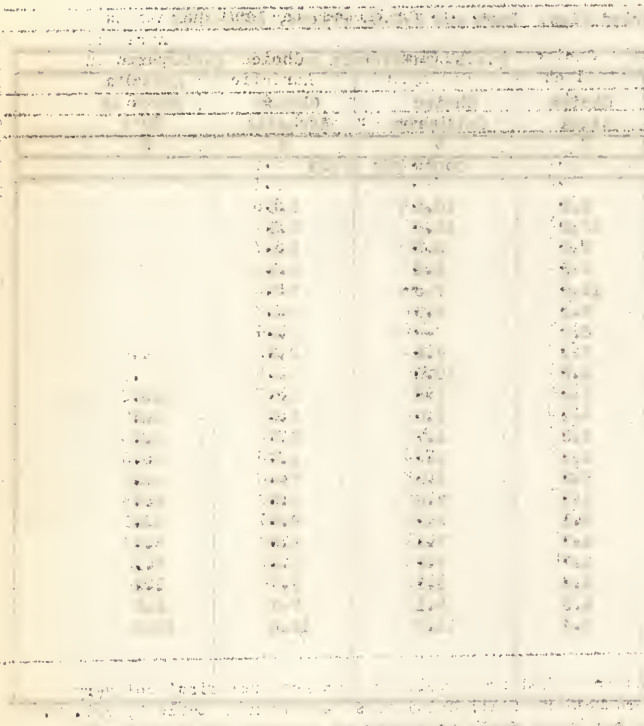

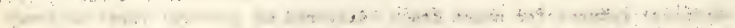

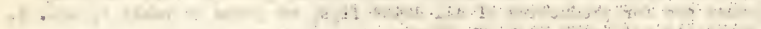

$$
\begin{aligned}
& \therefore \quad \therefore \text { a }
\end{aligned}
$$

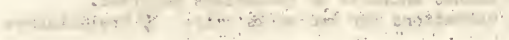

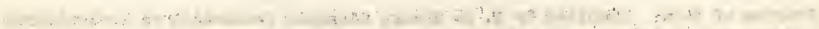
. . . .

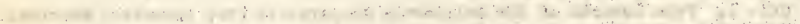

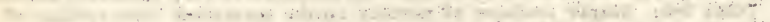

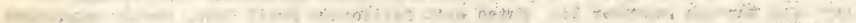

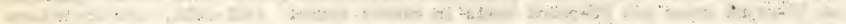

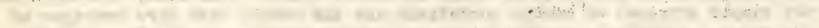

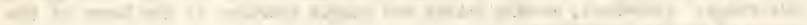

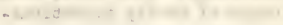


FIGS

Table 5

California Dried Fig Production by Varieties, 1932-1941

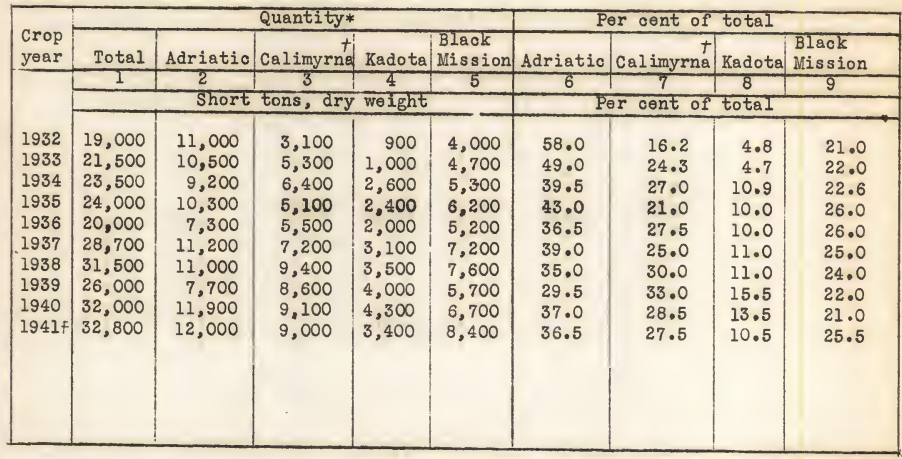

* Total of merchantable and nonmerchantable production.

+ Calimyrna estirates may be slightly too low as a larger proportion is usually culls, which are probably less completely and accurately reported by varieties than the merchantable tonnage.

7-Data are preliminary estimates.

Sources of data: Compiled by S. W. Shear, Giannini Foundation of Agrioultural Economios, University of California.

Col. 1: From reports of the California Cooperative crop Reporting Servioe, except 1934 data are revised estimates based upon a total of 23,442 tons compiled by the Dried Fruit Assooiation of California.

Cols. 2-5: Estimated by $S$. W. Shear by applying percentages in cols. 6-9 to total production in col. 1. Data are given to nearest hundred tons only.

Cols. 6-9: Data for 1932-1936 based largely upon data compiled by the Dried Fruit Association of California. 1937-1941 based on data from the California Fig Institute and the Fig Proration Zone. 


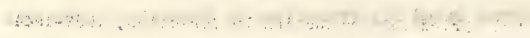

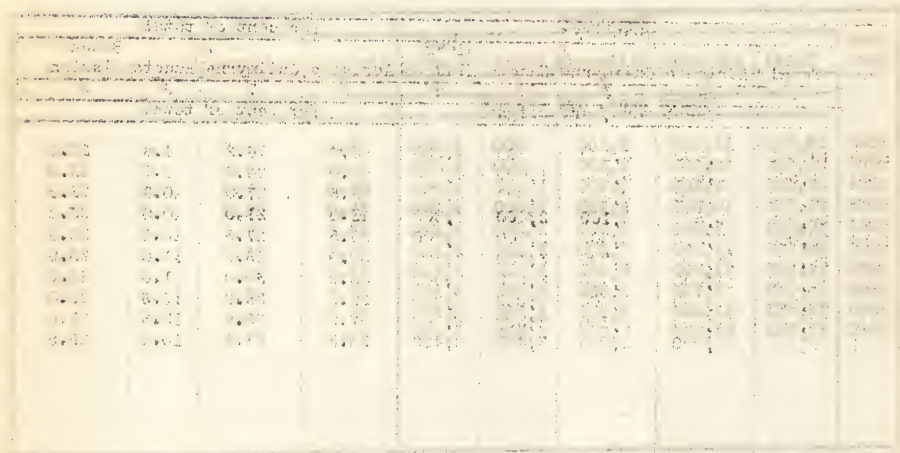

-

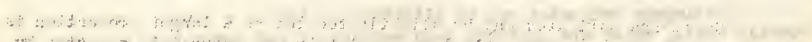

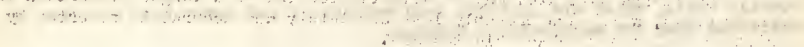

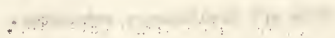

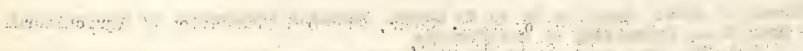

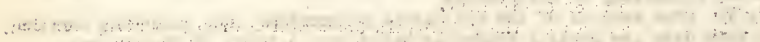

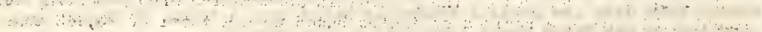

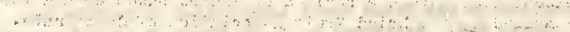

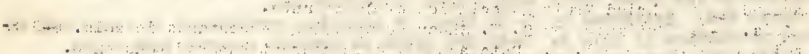

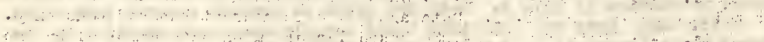
, a 
Table 6

Dried Figs: United States Merohantable* Production, Imports, Apparent Consumption and California Farm Price, 1919-1941

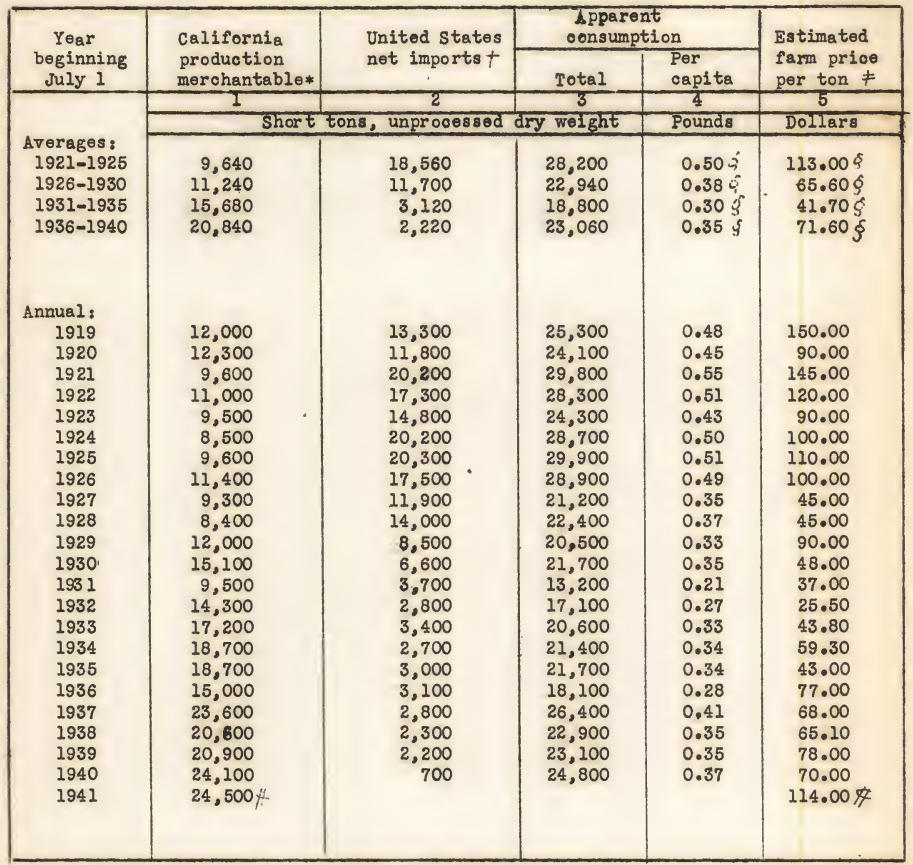

*Prior to 1927 include smell unestimated quantities of nonmerchantable figs. tData 1919-1932 imports minus reexports; 1933-1940 imports for consumption. FGrowers' returns for naked fruit delivered at growers' first delivery point; prices are for production of all dried figs, as given in table 2, col. 3. Ssimple averages. $\$$ Preliminary estimates.

Sources of data: Compiled by S. W. Shear, Giapnini Foundation of Agrioultural Bconomics, Unitersity of California.

Col. 1: From reports of the California Crop Reporting Service and the Dried Fruit Association of California.

Col 2, Based on data from U. S. Monthly summary of Foreign Commerce. Col 3: Col. 1 plus col. 2. Col. 4: Col.3 divided by United States population figures of January 1. Col. 5: From the Reports of Cal. Crop Rpt. Ser. 


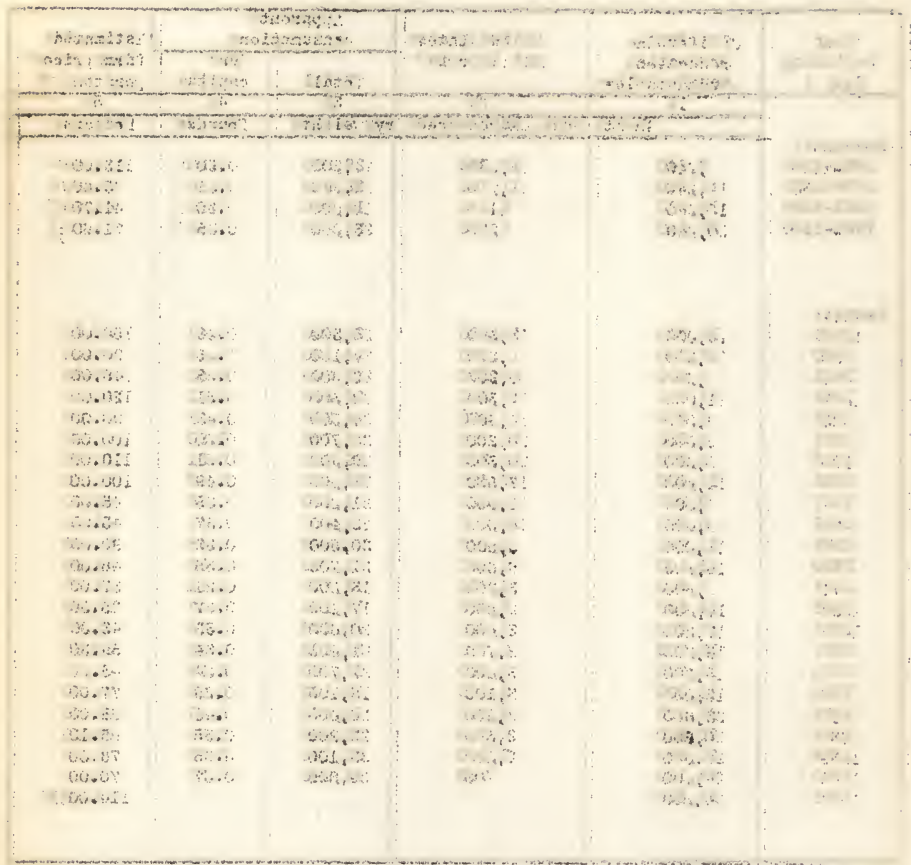

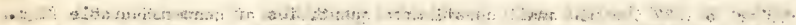

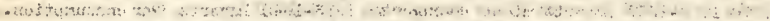

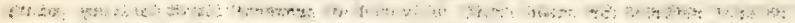

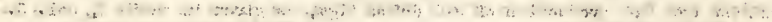
.

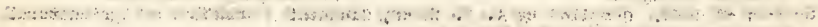

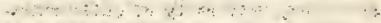

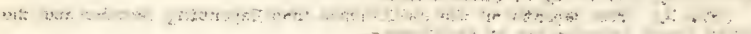

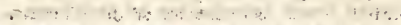

-

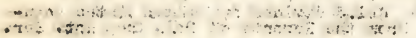

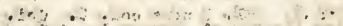

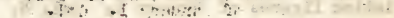


Table 7. Dried Figs: United States Importstby

Chief Countries of Origin, 1923-1940

\begin{tabular}{|c|c|c|c|c|c|c|}
\hline $\begin{array}{l}\text { Year begin- } \\
\text { ning July } 1\end{array}$ & $\begin{array}{l}\text { Total } \\
\text { imports }\end{array}$ & Turkey $\neq$ & Greece & Italy & Portugal & $\begin{array}{c}\text { Other } \\
\text { countries }\end{array}$ \\
\hline & $T$ & 2 & 3 & 4 & 5 & 6 \\
\hline & \multicolumn{2}{|r|}{ Shor } & tons, $n$ & declar & \multicolumn{2}{|c|}{ dry import weight } \\
\hline \multicolumn{7}{|l|}{ Annual: } \\
\hline & 15,834 & 9,845 & 2,228 & 763 & 1,933 & 1,065 \\
\hline 1924 & 22,630 & 11,078 & 3,798 & 1,897 & 2,397 & 3,460 \\
\hline 1925 & 21,841 & 10,295 & 2,308 & 1,861 & 4,183 & 3,194 \\
\hline 1926 & 19,752 & 11,135 & 3,421 & 1,652 & 1,393 & 2,151 \\
\hline 1927 & 15,730 & 8,283 & 1,232 & 972 & 2,967 & 2,276 \\
\hline 1928 & 17,782 & 11,209 & 2,455 & 679 & 2,202 & 1,237 \\
\hline 1929 & 10,959 & 6,392 & 3,042 & 321 & 467 & 737 \\
\hline 1930 & 7,413 & 4,999 & 1,467 & 509 & 422 & 16 \\
\hline 1931 & 4,348 & 3,125 & 590 & 390 & 199 & 44 \\
\hline 1932 & 3,019 & 2,150 & 484 & 354 & 15 & 16 \\
\hline 1933 & 3,543 & 2,591 & 519 & 344 & 71 & 18 \\
\hline 1934 & 2,828 & 1,525 & 759 & 474 & 35 & 35 \\
\hline 1935 & 3,191 & 1,761 & 1,018 & 302 & 58 & 52 \\
\hline 1936 & 3,302 & 1,511 & 981 & 762 & 45 & 3 \\
\hline 1937 & 2,946 & 963 & 1,415 & 523 & 31 & 14 \\
\hline 1938 & 2,416 & 860 & 896 & 643 & 10 & 7 \\
\hline 1939 & 2,323 & 804 & 1,346 & 155 & 17 & 1 \\
\hline 1940 & 785 & 333 & 407 & 0 & 45 & 0 \\
\hline \multirow{5}{*}{$\begin{array}{c}\text { Averages: } \\
1909-1913 \\
1924-1928 \\
1930-1933 \\
1934-1938\end{array}$} & & & & & & \\
\hline & 9,571 & 7,264 & 1,031 & 289 & 197 & 790 \\
\hline & 19,547 & 10,400 & 2,643 & 1,412 & 2,628 & 2,464 \\
\hline & 4,581 & 3,216 & 765 & 399 & 177 & 24 \\
\hline & 2,937 & 1,324 & 1,014 & 541 & 36 & 22 \\
\hline \multirow{5}{*}{$\begin{array}{l}\text { Averages : } \\
1909-1913 \\
1924-1928 \\
1930-1933 \\
1934-1938\end{array}$} & \multirow{2}{*}{\multicolumn{6}{|c|}{ Per cent of total }} \\
\hline & & & & & & \multirow{5}{*}{$\begin{array}{r}8.2 \\
12.6 \\
0.5 \\
0.8\end{array}$} \\
\hline & 100.0 & $\begin{array}{l}75.9 \\
53.2\end{array}$ & 10.8 & 3.0 & $\begin{array}{r}2.1 \\
13.5\end{array}$ & \\
\hline & 100.0 & 70.2 & 13.5 & $7 \cdot 2$ & 13.5 & \\
\hline & 100.0 & 45.1 & $\begin{array}{l}16.7 \\
34.5\end{array}$ & $\begin{array}{r}8.7 \\
18.4\end{array}$ & $\begin{array}{l}3.9 \\
1.2\end{array}$ & \\
\hline & & & & & & \\
\hline & & & & & & \\
\hline
\end{tabular}

*Imports are of all figs -- dried, fresh, preserved, etc., but, as far as is known, imports are practically all dried figs.

+ Imports for consumption only beginning January 1, 1934; previous data are total imports some of which were reexported.

* Includes only Turkey in Asia in 1923-1925, but Turkey in Europe and Asia both for other years. Most Turkish figs are exported from Smyrna.

Source of data: Compiled by S. W. Shear, Giannini Foundation of Agricultural

Economics, from official data of U.S. Dept. of Commerce from its reports

direct or from U. S. Dept. Agr. Yearbooks . 
$\therefore \quad \therefore \quad \therefore \quad \therefore$

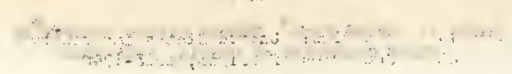

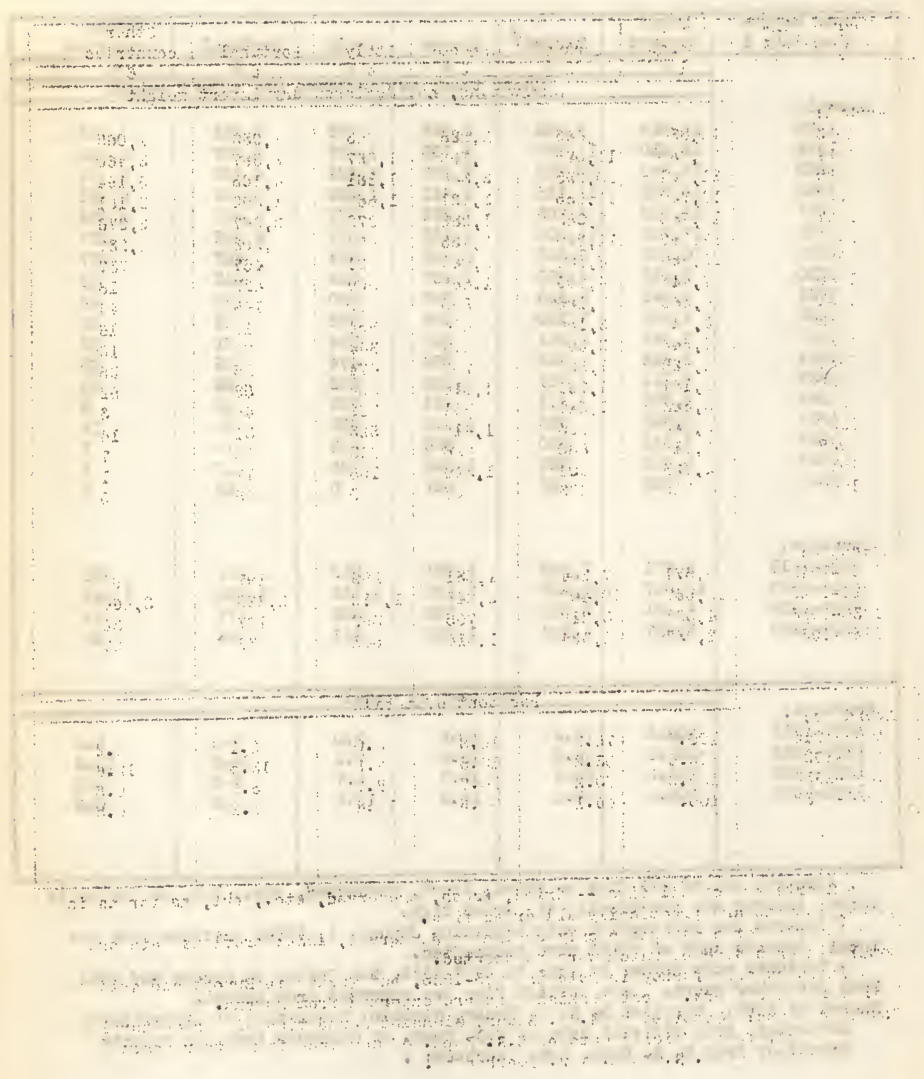


January, 1942

64.

FIGS

Table 8

Dried Fig Exports of Chief Mediterranean Producing Countries, 1920-1938*

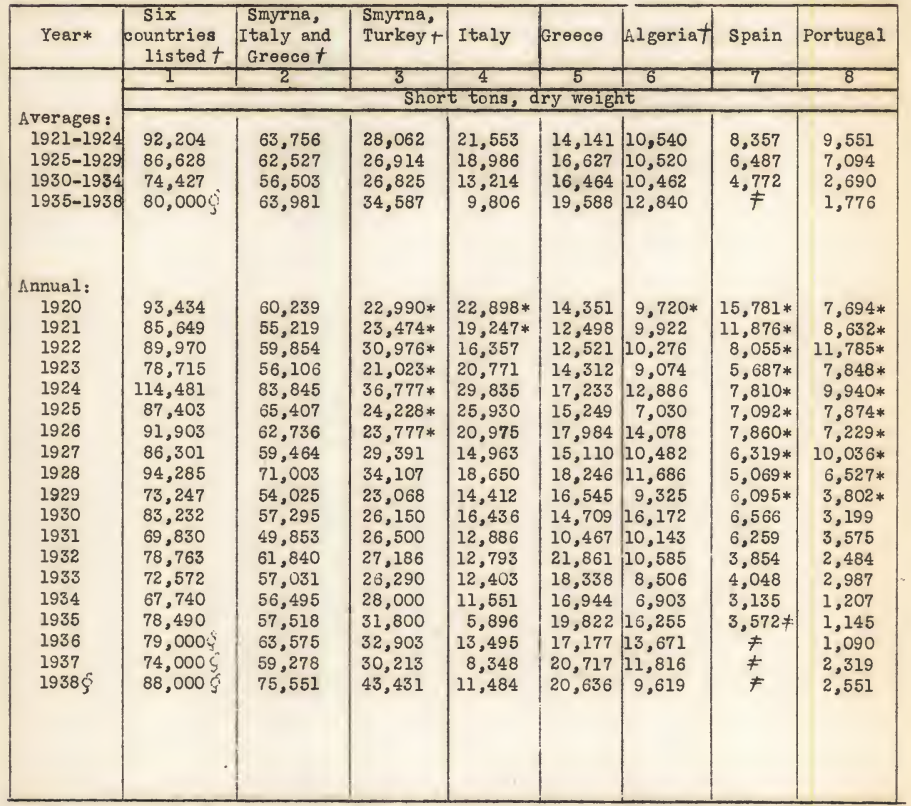

* Data marked with asterisk are for oalendar years; Algeria for years beginning July 1 ; all other for approximate crop years beginning August 1.

+ Some low grade inedible or horda figs are included in Smyrna and :.lgeria data in recent years and may be for earlier years for all countries.

F To June 30, 1936, only. Later data not available.

6 Preliminary data; totals in col. 1 for 1936-1938 given in round thousands assuming less than 1,000 tons exported from Spain annually 1936-1938.

Sources of data: Compiled by S. W. Shear, Giannini Foundation of Agricultural

Economics, University of California, from data compiled by office of Foreign Agricultural Relations, United States Department of igriculture. Some of these data have been published by the Federal-state Market News Service. 


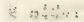

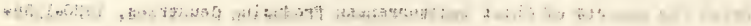

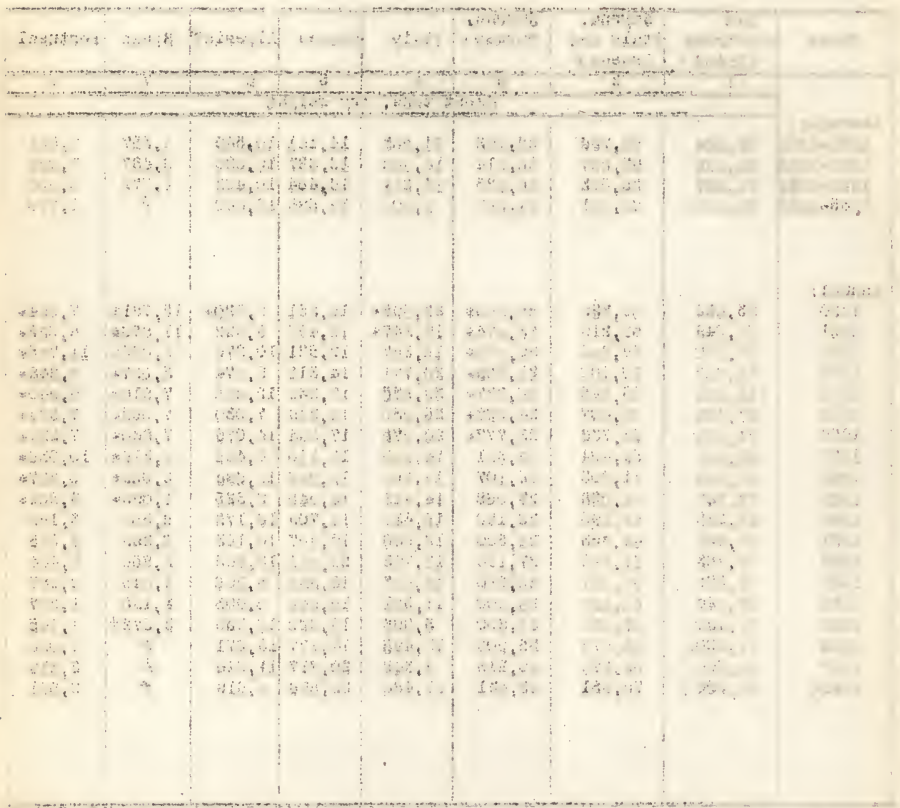

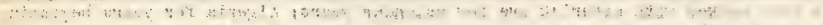

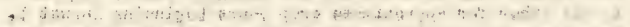

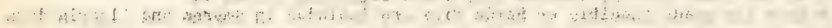

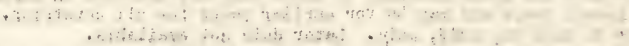

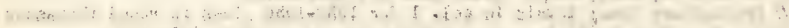

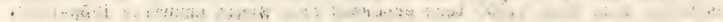

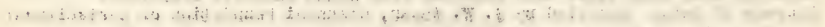

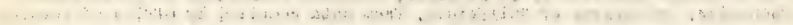

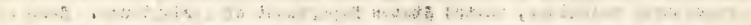

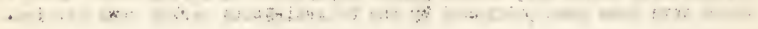


University of California, College of A\&riculture

Agricultural Experiment Station, Berkeley, January, 1942

DECIDUOUS FRUIT STATISTICS

GRAPIS

Table 1

Bearine dicreage of Californ ia Grapes, by Varietal* Classes 1919-1941

\begin{tabular}{|c|c|c|c|c|}
\hline Crop year & Total & Wine* & Table* & Raisin* \\
\hline & \multicolumn{4}{|c|}{ Acres } \\
\hline 1919 & 322,000 & 97,000 & 55,000 & 170,000 \\
\hline 1920 & $340^{\circ}, 000$ & 100,000 & 57,000 & 189,000 \\
\hline 1921 & 362,000 & 105,000 & 60,000 & 197,000 \\
\hline 1922 & 407,000 & 110,000 & 65,000 & 232,000 \\
\hline 1923 & 434,371 & 114,772 & 77,389 & 242,210 \\
\hline 1924 & 516,698 & 124,265 & 95,558 & 296,875 \\
\hline 1925 & 597,592 & 140,323 & 122,011 & 334,658 \\
\hline 1926 & 646,761 & 159,301 & 140,229 & 347,231 \\
\hline 1927 & 635,464 & 169,168 & 131,956 & 334,340 \\
\hline 1929 & 627,955 & 182,080 & 125,338 & 319,937 \\
\hline 1929 & 606,843 & 194,689 & 116,359 & 295,495 \\
\hline 1930 & 549,862 & 191,668 & 109,016 & 248,578 \\
\hline 1931 & 530,758 & $1 \approx 7,649$ & 99,957 & 243,152 \\
\hline 1932 & 525,040 & 187,092 & 97,571 & 240,377 \\
\hline 1933 & 504,552 & 182,514 & 88,923 & 233,115 \\
\hline 1934 & $499,186-$ & 181,578 & 87,278 & 230,330 \\
\hline 1935 & 491,100 & 177,500 & 78,000 & 235,000 \\
\hline 1936 & 468,468 & 162,899 & 77,609 & 227,960 \\
\hline 1937 & 481,689 & 167,864 & 79,109 & 234,716 \\
\hline 1938 & 487,453 & 169,826 & 79,240 & 238,387 \\
\hline 1939 & 488,428 & 109,206 & 79,127 & 240,095 \\
\hline 1940 & $482,345+$ & $164,409+$ & $79,246+$ & $238,090+$ \\
\hline $1941 \neq$ & 490,100 & 166,200 & 80,900 & 243,000 \\
\hline
\end{tabular}

* The ohief varieties included in each class by the crop Reporting Servioe in accordance with the most usual use for each variety are:

Faisin: Thompson Seedless, Muscat, Sultana, and Farte currant.

Table: Tokay, Nalaga, Emperor, Ted Nalaga, Cornichon, Almeria, Ribier.

Wine: Zinfandel, Alicante Bouschet, Carionane, Petit Sirah, Mission, liataro, and several milor black and white varieties.

+1940 nonbearing acres: total, 25,389; wine, 4,949; table, 5,088; and raisin, 15,652 .

キ Preliminary estimates.

Source of data: Compiled by $S$. Whear, Giannini Foundation of Africultural Economics, University of California, from Cali ornia Crop Reports. 
$\therefore \frac{1}{3}$

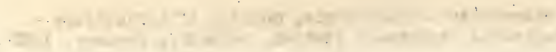

(1)

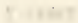

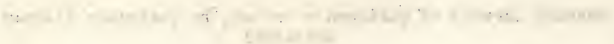

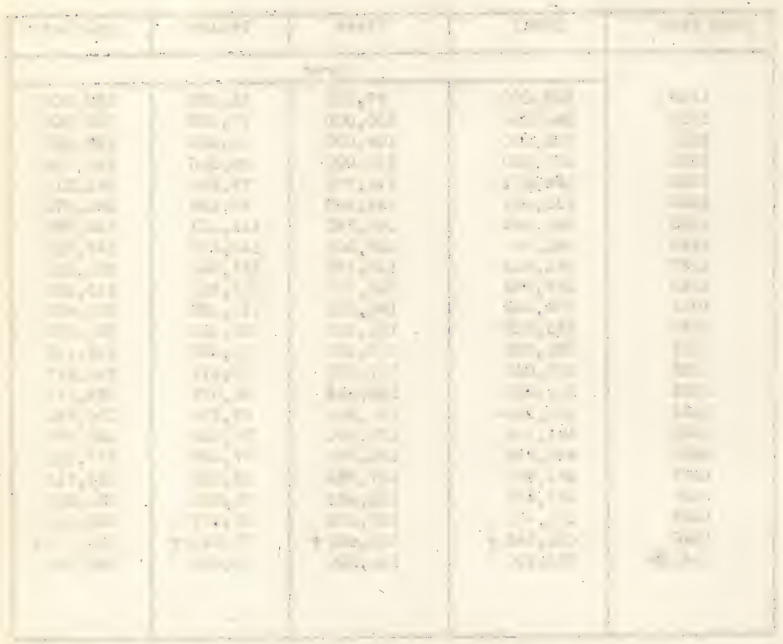

C. $\because$.

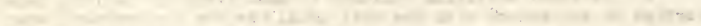

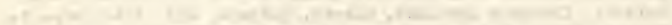

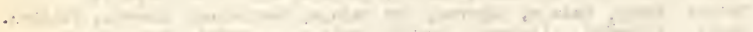

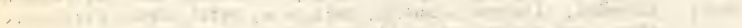

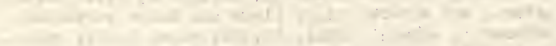

$\therefore \quad+2=-1,0$

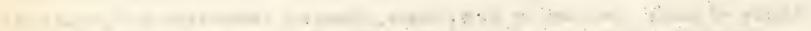
- 
Table 2. Grape Production* in California and United States, 1919-1941

\begin{tabular}{|c|c|c|c|c|c|c|}
\hline \multirow[b]{2}{*}{ Crop year } & \multirow[b]{2}{*}{$\begin{array}{l}\text { United States } \\
\text { total }\end{array}$} & \multirow[b]{2}{*}{$\begin{array}{l}\text { Other states } \\
\text { total }\end{array}$} & \multicolumn{4}{|c|}{ California } \\
\hline & & & Total & Raisin $T$ & Table + & vine $t$ \\
\hline \multirow{5}{*}{$\begin{array}{l}\text { Averages: } \\
1919-1923 \\
1924-1928 \\
1929-1933 \\
1934-1938\end{array}$} & & Thousands 0 & hort ton & fresh we & ht & \\
\hline & 1,730 & 219 & 1,511 & 887 & 225 & 399 \\
\hline & 2,333 & 236 & 2,097 & 1,248 & 426 & 423 \\
\hline & 2,071 & 289 & 1,782 & 1,074 & 303 & 405 \\
\hline & 2,369 & 250 & 2,119 & 1,189 & 372 & 558 \\
\hline \multicolumn{7}{|l|}{ Annual: } \\
\hline 1919 & 1,575 & 230 & 1,345 & 745 & 200 & 400 \\
\hline 1920 & 1,521 & 248 & 1,273 & 732 & 166 & 375 \\
\hline 1921 & 1,220 & 120 & 1,100 & 627 & 163 & 310 \\
\hline 1922 & 2,085 & 279 & 1,806 & 1,043 & 283 & 480 \\
\hline 1923 & 2,250 & 220 & 2,030 & 1,290 & 312 & 428 \\
\hline 1924 & 1,775 & 240 & 1,535 & 860 & 325 & 350 \\
\hline 1925 & 2,200 & 150 & 2,050 & 1,216 & 439 & 395 \\
\hline 1926 & 2,444 & 315 & 2,129 & 1,317 & 398 & 414 \\
\hline 1927 & 2,592 & 186 & 2,406 & 1,443 & 490 & 473 \\
\hline 1928 & 2,654 & 288 & 2,366 & 1,406 & 478 & 482 \\
\hline 1929 & 2,085 & 258 & 1,827 & 1,098 & 312 & 417 \\
\hline 1930 & 2,466 & 275 & 2,181 & 1,307 & 388 & 486 \\
\hline 1931 & 1,646 & 326 & 1,320 & 775 & 229 & 316 \\
\hline 1932 & 2,231 & 305 & 1,926 & 1,221 & 317 & 388 \\
\hline 1933 & 1,939 & 279 & 1,660 & 970 & 270 & 420 \\
\hline 1934 & 1,958 & 258 & 1,700 & 928 & 296 & 476 \\
\hline 1935 & 2,488 & 294 & 2,194 & 1,248 & 375 & 571 \\
\hline 1936 & 1,916 & 202 & 1,714 & 918 & 324 & 472 \\
\hline 1937 & 2,777 & 323 & 2,454 & 1,407 & 416 & 631 \\
\hline 1938 & 2,704 & 173 & 2,531 & 1,443 & 447 & 641 \\
\hline 1939 & 2,526 & 298 & 2,228 & 1,269 & 390 & 569 \\
\hline 1940 & 2,548 & 298 & 2,250 & 1,213 & 430 & 607 \\
\hline $1941 \neq$ & $2,785 \neq$ & 240 & $2,545 \neq$ & $1,510 \neq$ & $480 \neq$ & $555 \neq$ \\
\hline
\end{tabular}

* Includes harvested and unharvested production (see tables 6-9).

† The chief varieties included in each class by the crop Reporting Service in accordance with the most usual use for each variety are as follows: Raisin: Thompson Seedless, Huscat, Sultana, and Zante Currant. Table: Tokay, Malaga, Fmperor, Red Nalaga, Cornichon, Almeria, Ribier. Fine: Zinfandel, Alicante Bouschet, Carigmane, Petit Sirah, Hission, lataro, and several minor black and white varieties.

\# Unofficial revisions of California production involving data on commercial crush and dried raisin pack released by the Wine Institute and the Dried Fruit Association in Jarch, 1942. Preliminary estimates for other states are official. Sources of data:

Compiled by S. W. Shear, Giannini Foundation of Agricultural Economics, from United States and California Crop Reports, except 1941 estimates. 


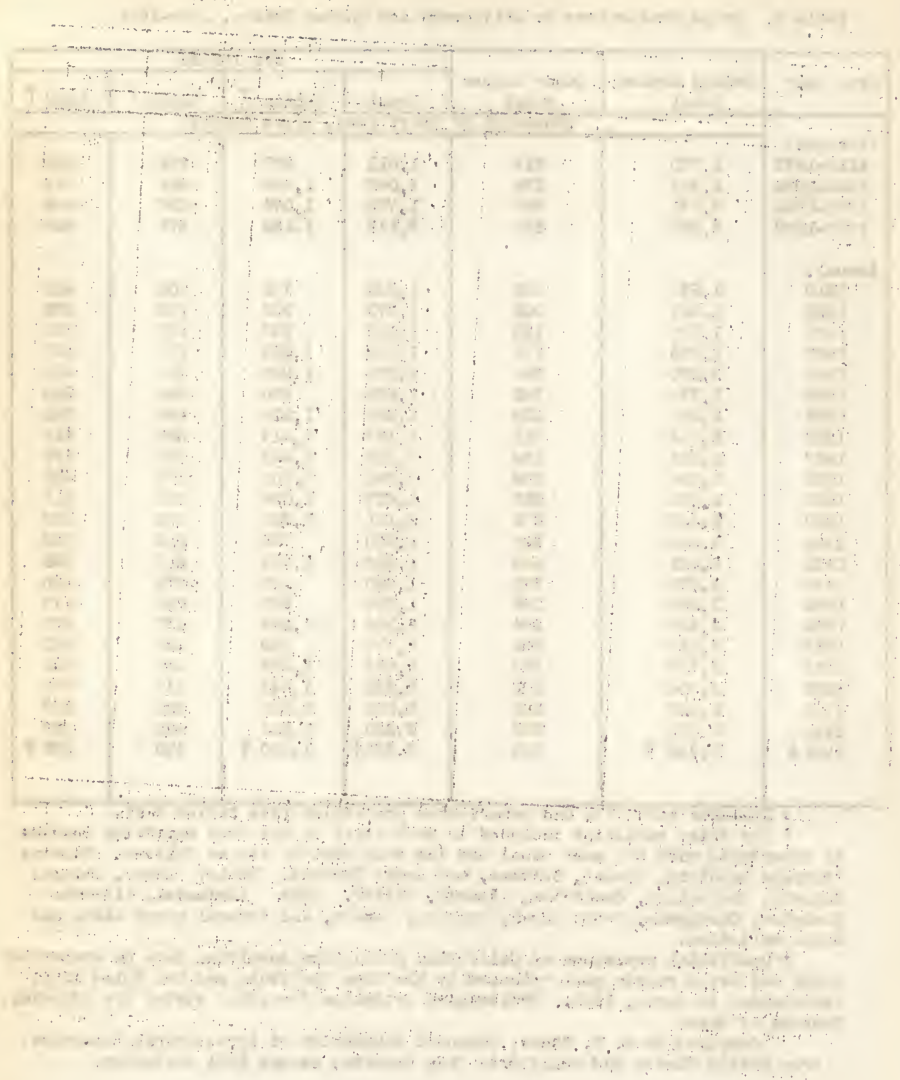


Table 3

Califormia Grapes: Yield Per Bearing, Acre and as Per Cent of Full Yield, by Varietal* Classes, 1919-1941

\begin{tabular}{|c|c|c|c|c|c|c|c|c|}
\hline \multirow[b]{2}{*}{ Crop year } & \multicolumn{4}{|c|}{ Yield per bearing acre } & \multirow{2}{*}{\multicolumn{4}{|c|}{\begin{tabular}{l|l|l|l} 
Nov. 1 per cent of full yield \\
Total & Table* & Tine* & Raisin*
\end{tabular}}} \\
\hline & Total & Table* & Wine* & Raisin* & & & & \\
\hline & \multicolumn{4}{|c|}{ Short tons } & \multicolumn{4}{|c|}{ Per cent } \\
\hline $\begin{array}{c}\text { Averages: } \\
1919-1923\end{array}$ & 4.0 & 3.5 & 3.8 & 4.3 & 87 & 84 & 89 & 89 \\
\hline $1924-1928$ & 3.5 & 3.5 & 2.7 & 3.8 & 77 & 73 & 81 & 77 \\
\hline $1929-1933$ & 3.3 & 2.9 & 2.1 & 4.3 & 69 & 68 & 70 & 69 \\
\hline $1934-1938$ & 4.4 & 4.7 & 3.3 & 5.1 & 76 & 76 & 81 & 75 \\
\hline \multicolumn{9}{|l|}{ Annual: } \\
\hline 1919 & 4.2 & 3.6 & 4.1 & 4.4 & 94 & 95 & 93 & 98 \\
\hline 1920 & 3.7 & 2.9 & 3.8 & 3.9 & 90 & 82 & 90 & 90 \\
\hline 1921 & 3.0 & 2.7 & 3.0 & 3.2 & 66 & 69 & 69 & 67 \\
\hline 1922 & 4.4 & 4.4 & 4.4 & 4.5 & 96 & 92 & 102 & 100 \\
\hline 1923 & 4.7 & 4.1 & 3.7 & 5.3 & 90 & 82 & 90 & 91 \\
\hline 1924 & 3.0 & 3.4 & 2.8 & 2.9 & 69 & 70 & 70 & 65 \\
\hline 1925 & 3.4 & 3.6 & 2.8 & 3.6 & 75 & 72 & 82 & 73 \\
\hline 1926 & 3.3 & 2.8 & 2.6 & 3.8 & 74 & 65 & 82 & 75 \\
\hline 1927 & 3.8 & 3.7 & 2.8 & 4.3 & 84 & 78 & 87 & 85 \\
\hline 1928 & 3.8 & 3.8 & 2.6 & 4.4 & 84 & 80 & 84 & 85 \\
\hline 1929 & 3.0 & 2.7 & 2.1 & 3.7 & 66 & 62 & 73 & 65 \\
\hline 1930 & 4.0 & 3.5 & 2.5 & 5.3 & 84 & 86 & 81 & 84 \\
\hline 1931 & 2.5 & 2.3 & 1.7 & 3.2 & 54 & 53 & 60 & 52 \\
\hline 1932 & 3.7 & 3.2 & 2.1 & 5.1 & 75 & 72 & 68 & 79 \\
\hline 1933 & 3.3 & 3.0 & 2.3 & 4.2 & 65 & 65 & 66 & 65 \\
\hline 1934 & 3.4 & 3.4 & 2.6 & 4.0 & 65 & 64 & 75 & 61 \\
\hline 1935 & 4.5 & 4.7 & 3.2 & 5.3 & 80 & 78 & 83 & 80 \\
\hline 1936 & 3.7 & 4.2 & 2.9 & 4.0 & 63 & 69 & 72 & 58 \\
\hline 1937 & 5.1 & 5.3 & 3.8 & 6.0 & 89 & 85 & 87 & 91 \\
\hline 1938 & 5.2 & 5.7 & 3.8 & 6.1 & 85 & 84 & 86 & 85 \\
\hline 1939 & 4.6 & 4.9 & 3.4 & 5.3 & 76 & 74 & 75 & 77 \\
\hline 1940 & 4.7 & 5.4 & 3.7 & 5.1 & 77 & 80 & 81 & 75 \\
\hline $1941 \neq$ & 5.2 & 5.9 & 3.3 & 5.2 & 83 & 75 & 81 & 86 \\
\hline
\end{tabular}

* The chief varieties included in each class by the Crop Reporting Service in accordance with the most usual use for each variety are as follows:

Raisin: Thompson Seedless, luscat, Sultana, and Zante Currant.

Table: Tokay, Illaga, Tmperor, Red lalaga, Cornichon, Almeria, Ribier.

Wine: Zinfandel, Alicante Bouschet, Carignane, Petit Sirah, Ifission, Mataro, and several minor black and white varieties.

+ Simple averages.

F Preliminary estimates; yields per acre based on unofficial production estimates.

Source of data:

Compiled by S. W. Shear, Giannini Foundation of Agricultural Fconomics, University of California, based upon U. S. and California Crop Reports. 


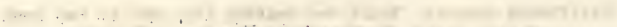

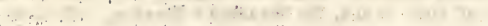

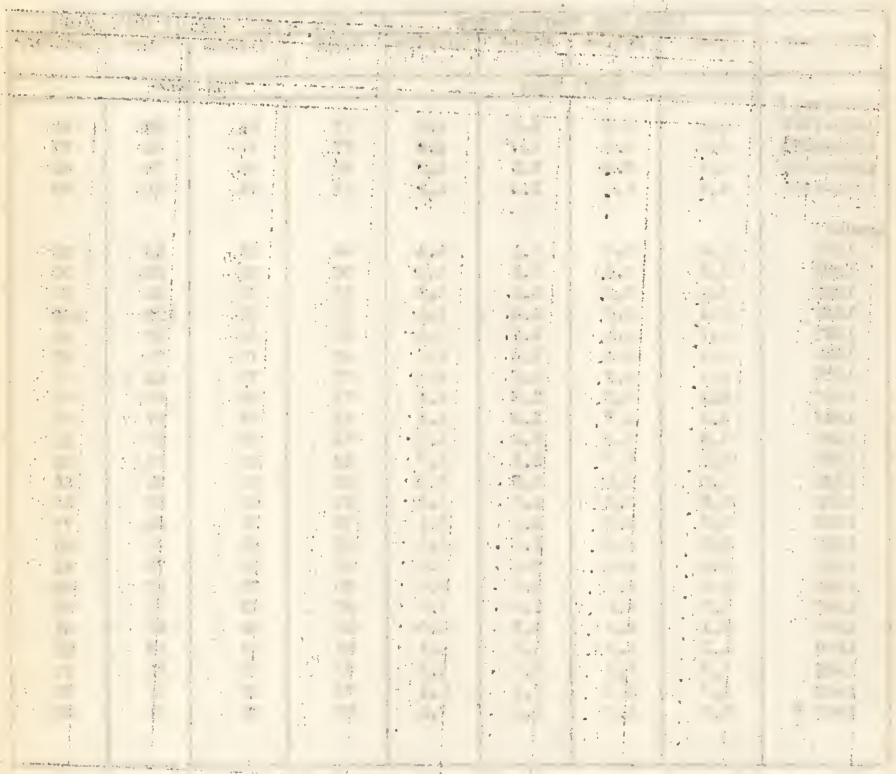

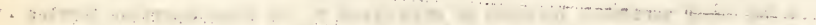

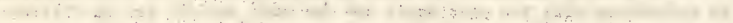

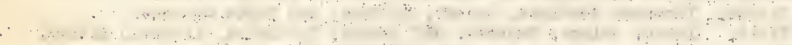

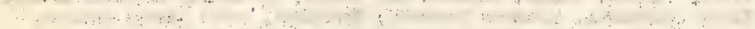

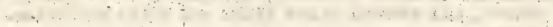




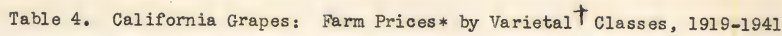

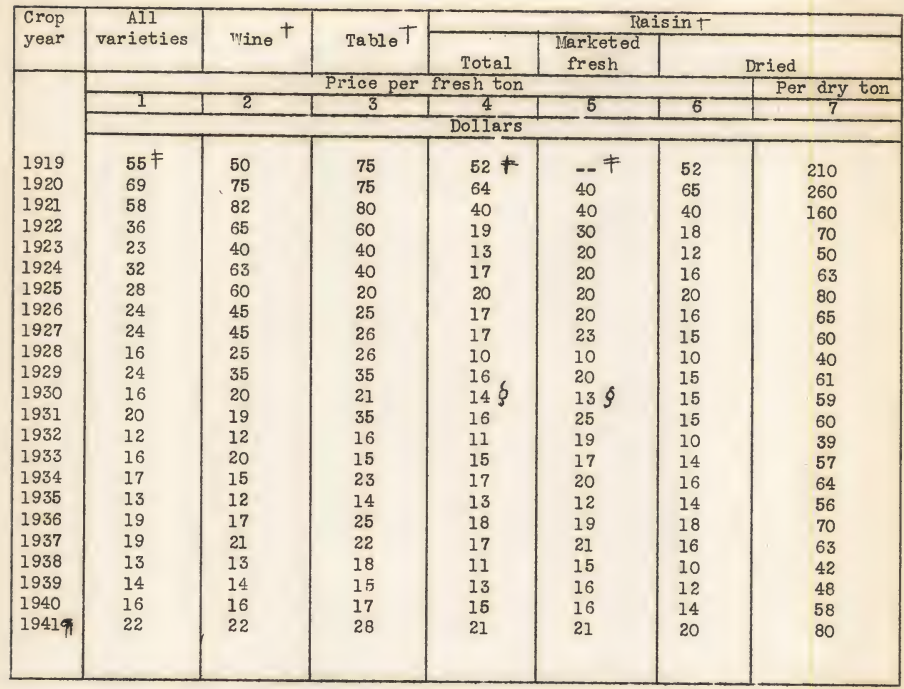

* Growers' returns for naked fruit at grovers' first delivery point.

+ The chief varieties included in each class by the Crop Reporting Service in accordance with the most usual use for each variety are:

Raisin: Thompson Seedless, Juscat, Sultana, and Zante Currant.

Table: Tokay, Malaga, Fmperor, Red alaga, Cornichon, Almeria, Ribier.

Wine: Zinfandel, Alicante Bouschet, Carignane, Petit Sirah, Mission, lataro, and several minor black and white varieties.

F Excludes price of 13,000 tons of raisin grapes marketed fresh for which price unavailable.

$\oint$ Includes returns from Control Board for unharvested grapes.

98 Preliminary.

Sources of data:

Data compiled by S. W. Shear, Giannini Foundation of Agricultural Foonomics, University of California, from official reports of United States and California Cooperative Crop Reporting Service, except col. 6, which is calculated by dividing items in col. 7 by 4 , and cols. 1 and 4 , which are weighted averages of prices in other colunns. 
$\therefore$

$\therefore \because, \cdots, \cdots+\cdots t$,

. . . .

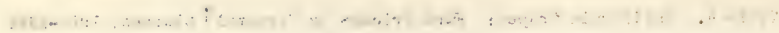

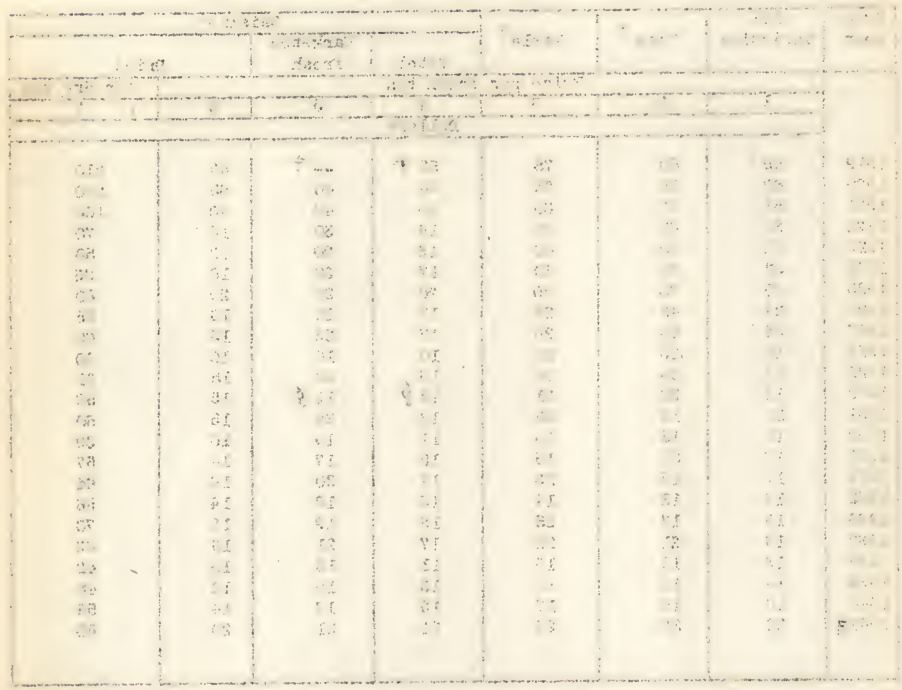

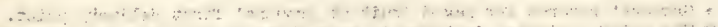

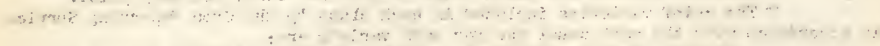
.

,

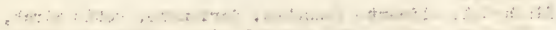

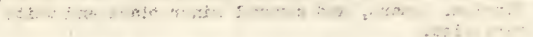

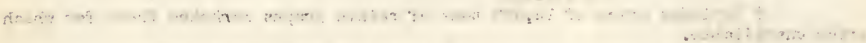

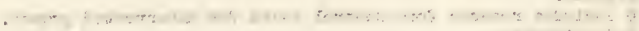

$\cdots \quad \cdots, \cdots, \cdots, \cdots$

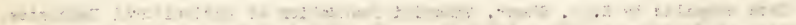

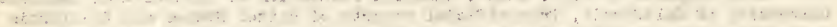

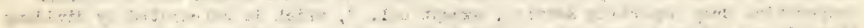

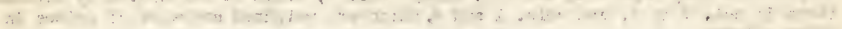

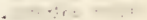


Table 5

California Grapes: Farm Value* by Varietal Classes, 1919-1941

\begin{tabular}{|c|c|c|c|c|c|c|}
\hline \multirow[b]{2}{*}{ Crop year } & \multirow[b]{2}{*}{ Total } & \multirow[b]{2}{*}{ गाinet } & \multirow[b]{2}{*}{ mable $T$} & \multicolumn{3}{|c|}{ Risint } \\
\hline & & & & Total & $\begin{array}{l}\text { Narketed } \\
\text { fresh }\end{array}$ & Dried \\
\hline & I & 2 & 3 & 4 & 5 & 6 \\
\hline & \multicolumn{6}{|c|}{ Thousands of dollars } \\
\hline 1919 & $73,430 \neq$ & 20,000 & 15,000 & $38,430 \neq$ & * & 38,430 \\
\hline 1920 & 87,555 & 28,125 & 12,450 & 46,980 & 960 & 46,020 \\
\hline 1921 & 63,540 & 25,420 & 13,040 & 25,080 & 1,880 & 23,200 \\
\hline 1922 & 61,470 & 29,250 & 12,780 & 19,440 & 2,850 & 16,590 \\
\hline 1923 & 46,700 & 17,120 & 12,480 & 17,100 & 2,600 & 14,500 \\
\hline 1924 & 49,360 & 22,050 & 13,000 & 14,310 & 3,600 & 10,710 \\
\hline 1925 & 54,040 & 23,700 & 6,780 & 23,560 & 7,560 & 16,000 \\
\hline 1926 & 50,465 & 18,630 & 9,575 & 22,260 & 4,580 & 17,680 \\
\hline 1927 & 55,530 & 21,285 & 10,176 & 24,069 & 6,969 & 17,100 \\
\hline 1928 & 35,538 & 11,600 & 10,478 & 13,460 & 3,020 & 10,440 \\
\hline 1929 & 43,390 & 14,595 & 10,920 & 17,875 & 4,760 & 13,115 \\
\hline 1930 & 33,649 & 8,920 & 6,540 & 18,189 & 6,861 & 11,328 \\
\hline 1931 & 26,503 & 5,814 & 8,084 & 12,605 & 2,465 & 10,140 \\
\hline 1932 & 20,765 & 4,356 & 3,344 & 13,065 & 2,847 & 10,218 \\
\hline 1933 & 26,727 & 8,295 & 3,952 & 14,480 & 3,287 & 11,193 \\
\hline 1934 & 29,520 & 7,021 & 6,675 & 15,824 & 4,880 & 10,944 \\
\hline 1935 & 28,344 & 6,709 & 5,250 & 16,385 & 5,058 & 11,327 \\
\hline 1936 & 32,741 & 8,166 & 8,213 & 16,362 & 3,686 & 12,676 \\
\hline 1937 & 46,690 & 13,251 & 9,069 & 24,370 & 8,883 & 15,487 \\
\hline 1938 & 32,459 & 8,077 & 7,957 & 16,425 & 4,245 & 12,180 \\
\hline 1939 & 30,523 & 8,080 & 5,850 & 16,593 & 4,711 & 11,882 \\
\hline 1940 & 35,125 & 9,894 & 7,181 & 18,050 & 8,200 & 9,850 \\
\hline 1941 \$ & 56,789 & 12,210 & 13,440 & 31,139 & 14,356 & 16,783 \\
\hline
\end{tabular}

* Growers' returns for naked fruit at growers' first delivery point.

t The chief varieties included in each class by the Crop Reporting Service in accordance with the most usual use for each variety are:

Raisin: Thompson Seedless, liuscat, Sultana, and Zante Currant.

Table: Tokay, ifalaga, Emperor, Red íalaga, Cornichon, Almeria, and Ribier.

Wine: Zinfandel, Alicante Bouschet, Carignane, Petit Sirah, Ilission, ilataro, and several minor black and white varieties.

F Excludes value of 13,000 tons of raisin grapes marketed fresh for which data not available.

$\oint$ Preliminary; partly based on unofficial production estimates.

Sources of data:

Compiled by S. W. Shear, Giannini Foundation of Agricultural Economics,

University of California, based on official reports of United States and

California Cooperative Crop Reporting Service. 
$\because \ldots+\cdots$

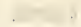

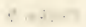

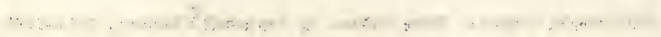

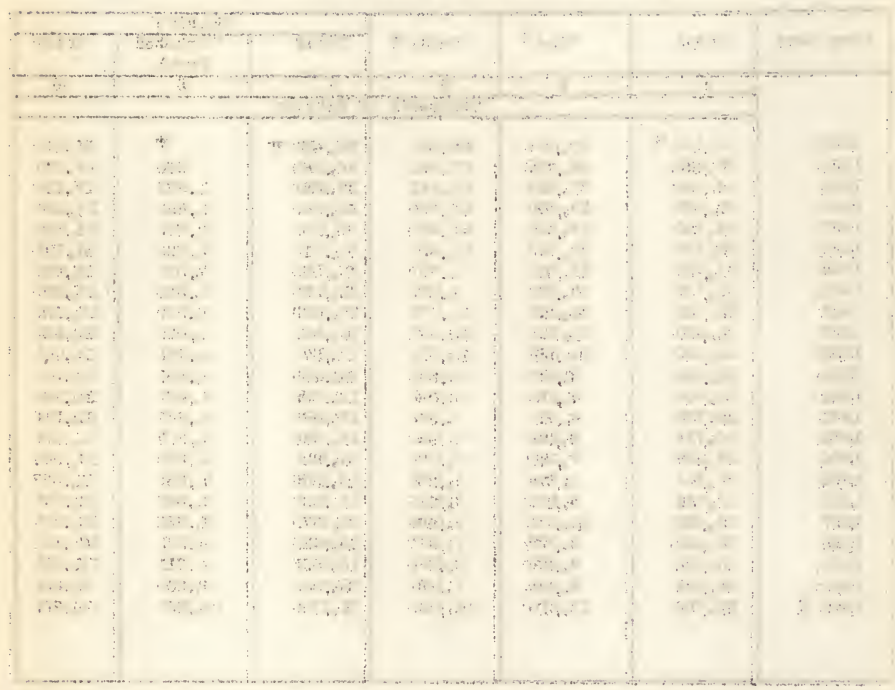

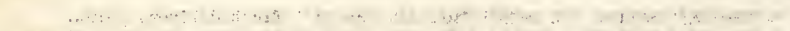

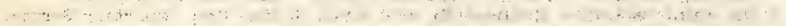

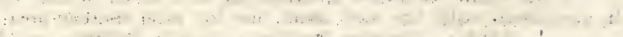

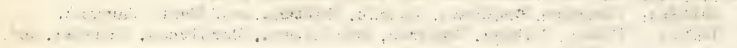

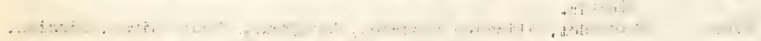

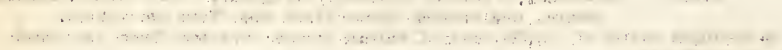

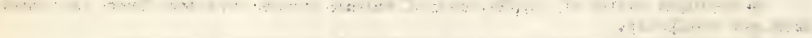

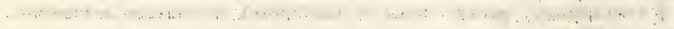

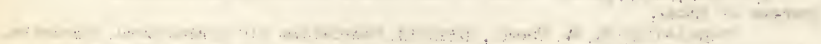

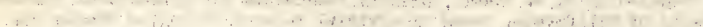

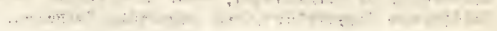


GRAPES

Table 6. California Production and Utilization of all Varieties of Grapes, 1927-1941

\begin{tabular}{|c|c|c|c|c|c|c|c|c|c|c|c|}
\hline \multirow{3}{*}{$\begin{array}{l}\text { Crop } \\
\text { year }\end{array}$} & \multirow{3}{*}{$\begin{array}{l}\text { Hervested* } \\
\text { production }\end{array}$} & \multirow[b]{3}{*}{ Canned } & \multirow[b]{3}{*}{ Dried $T$} & \multicolumn{5}{|c|}{ Used fresh for juice } & \multicolumn{3}{|c|}{ Fresh table use } \\
\hline & & & & \multirow[b]{2}{*}{ Total } & \multirow{3}{*}{$\begin{array}{c}\text { Commercial } \\
\text { drush }\end{array}$} & \multicolumn{3}{|c|}{ Other } & \multirow[b]{2}{*}{$\begin{array}{l}\text { Inter- } \\
\text { state }\end{array}$} & \multirow[b]{2}{*}{$\begin{array}{r}\text { Within } \\
\text { state }\end{array}$} & \multirow[b]{2}{*}{ Total } \\
\hline & & & & & & Total & $\begin{array}{r}\text { Within } \\
\text { state } \\
\end{array}$ & $\begin{array}{l}\text { Inter- } \\
\text { state }\end{array}$ & & & \\
\hline & & & & & & sh weif & & & & & \\
\hline \multicolumn{9}{|l|}{ Averages: $\#$} & & & \\
\hline $1927-1929$ & $2,115,800$ & 2,100 & $1,045,300$ & 698,800 & 76,300 & 622,500 & 48,700 & 573,800 & 335,000 & 34,600 & 369,600 \\
\hline $1930-1932$ & $1,610,000$ & 700 & 848,900 & 499,700 & 69,200 & 430,500 & 67,500 & 363,000 & 223,900 & 36,800 & 260,700 \\
\hline $1934-1938$ & $2,118,600$ & 4,100 & 881,500 & 946,700 & 736,800 & 209,900 & 18,900 & 191,000 & 242,000 & 44,300 & 286,300 \\
\hline \multicolumn{12}{|l|}{ Annual: } \\
\hline 1927 & $2,307,400^{n}$ & 2,400 & $1,166,000$ & 755,900 & 75,000 & 680,900 & 44,500 & 636,400 & 347,600 & 35,500 & 383,100 \\
\hline 1928 & $2,213,000^{*}$ & 1,600 & $1,072,000$ & 734,000 & 103,000 & 631,000 & 49,500 & 581,500 & 372,500 & 32,900 & 405,400 \\
\hline 1929 & $1,827,000$ & 2,300 & 898,000 & 606,500 & 51,000 & 555,500 & 52,200 & 503,300 & 284,700 & 35,500 & 320,200 \\
\hline 1930 & $1,748,000^{*}$ & 1,300 & 782,100 & 648,200 & 74,100 & 574,100 & 74,300 & 499,800 & 281,500 & 34,900 & 316,400 \\
\hline 1931 & $1,310,000^{*}$ & 300 & 702,400 & 371,900 & 34,600 & 337,300 & 68,100 & 269,200 & 204,300 & 31,100 & 235,400 \\
\hline 1932 & $1,772,000$ & 500 & $1,062,000$ & 479,100 & 99,000 & 380,100 & 60,100 & 320,000 & 186,000 & 44,400 & 230,400 \\
\hline 1933 & $1,657,000^{*}$ & 970 & 793,000 & 661,800 & 444,000 & 217,800 & 33,400 & 184,400 & 163,700 & 37,530 & 201,230 \\
\hline 1934 & $1,700,000$ & 2,200 & 695,200 & 739,200 & 530,000 & 209,200 & 27,000 & 182,200 & 220,900 & 42,500 & 263,400 \\
\hline 1935 & $2,194,000$ & 2,400 & 816,000 & $1,123,100$ & 887,000 & 236,100 & 18,700 & 217,400 & 210,100 & 42,400 & 252,500 \\
\hline 1936 & $1,714,000$ & 3,200 & 733,600 & 687,300 & 494,000 & 193,300 & 20,300 & 173,000 & 247,400 & 42,500 & 289,900 \\
\hline 1937 & $2,454,000$ & 7,500 & 994,000 & $1,136,100$ & 911,000 & 225,100 & 11,300 & 213,800 & 270,300 & 46,100 & 316,400 \\
\hline 1938 & $2,531,000$ & 5,000 & $1,168,800$ & $1,048,000$ & 862,000 & 186,000 & 17,200 & 168,800 & 261,400 & 47,800 & 309,200 \\
\hline 1939 & $2,228,000$ & 12,000 & 988,600 & 934,800 & 712,000 & 222,800 & 12,400 & 210,400 & 240,400 & 52,200 & 292,600 \\
\hline 1940 & $2,250,000$ & 11,300 & 688,600 & $1,223,200$ & 996,000 & 227,200 & 11,100 & 216,100 & 271,100 & 55,800 & 326,900 \\
\hline $1941 \oint$ & $2,545,000$ & 12,000 & 840,000 & $1,349,300$ & $1,126,000$ & 223,300 & 12,000 & 211,300 & 297,700 & 46,000 & 343,700 \\
\hline
\end{tabular}

* Excludes unharvested tons: $1927,98,600 ; 1928,153,000 ; 1930,433,000 ; 1931,10,000 ; 1932,154,000 ; 1933,3,000$.

+ Dried wine and table grapes included probably finally used for juice and a few raisins.

F Averages do not equal the sum of similar averages, tables $7-9$, due to minor adjustments in subtotals,

$\oint$ tnoficial revisions of production and utilization involving data on the commercial crush and the dried raisin pack released by the Wine Institute and the Dried Fruit Association in March, 1942.

Sources of data:

Compiled by S. W. Shear, Giannini Foundation of Agricultural Economics, University of California. Data

largely based on reports of California Crop Reporting Service and of Federal-State Market News Service, except

fresh juice and table stock in all years and commercial crush 1927-1929 and unharvested in 1927 made by

S. W. Shear. 


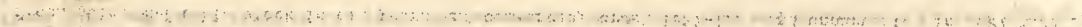

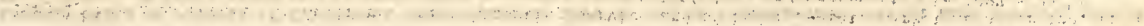

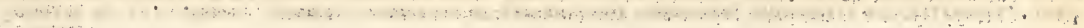

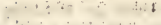

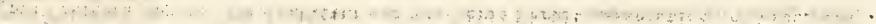

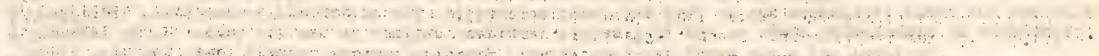
. A

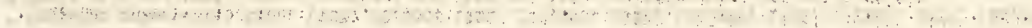

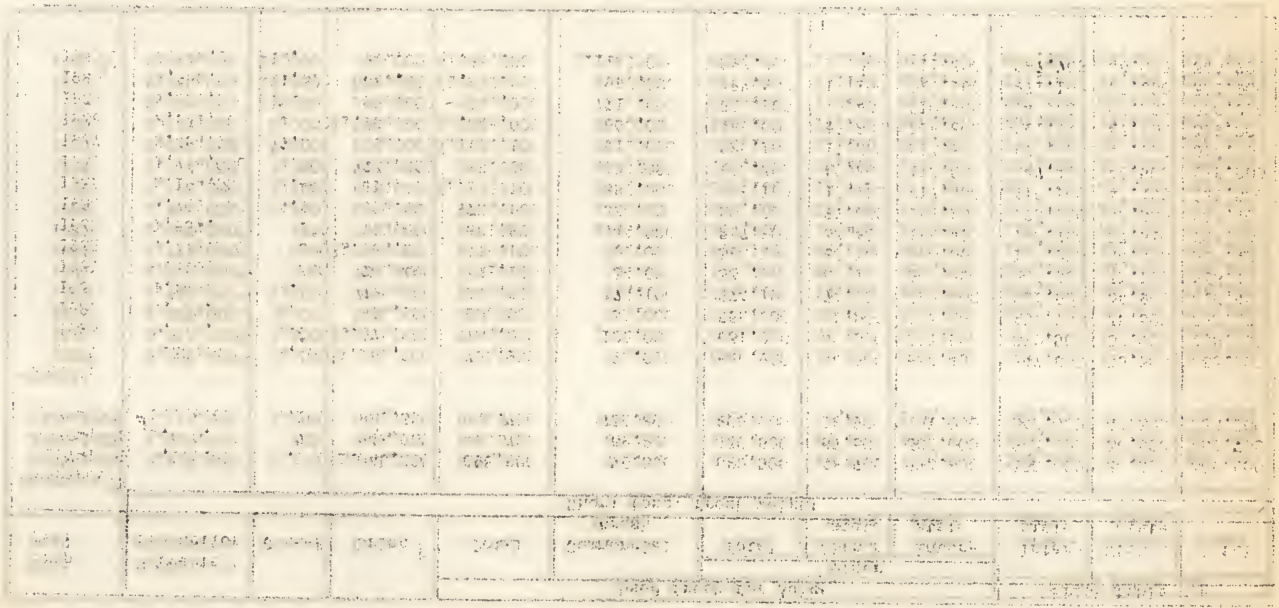

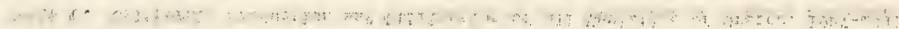

3. $: 5$. 
GRAPES

January, 1942

Table 7. California Production and Utilization of Raisin Grape Varieties, 1927-1941

\begin{tabular}{|c|c|c|c|c|c|c|c|c|c|}
\hline \multirow[b]{2}{*}{ Crop year } & \multirow[b]{2}{*}{$\begin{array}{l}\text { Harvested" } \\
\text { production }\end{array}$} & \multirow[b]{2}{*}{ Canned } & \multirow[b]{2}{*}{ Dried $t$} & \multicolumn{3}{|c|}{ Used fresh for juice $t$} & \multicolumn{3}{|c|}{ Fresh table use } \\
\hline & & & & Total $\neq$ & $\begin{array}{c}\text { Commercial } \\
\text { crush }\end{array}$ & Interstate $\oint$ & Interstate $\oint$ & $\begin{array}{l}\text { Within } \\
\text { state }\end{array}$ & Total \\
\hline \multirow{2}{*}{\multicolumn{10}{|c|}{ Averages: }} \\
\hline & & & & & & & & & \\
\hline $1927-1929$ & $1,295,700$ & 2,100 & $1,014,700$ & 201,000 & 21,500 & 169,600 & 66,700 & 11,200 & 77,900 \\
\hline $1930-1932$ & 987,200 & 700 & 830,400 & 95,000 & 12,900 & 73,300 & 49,700 & 11,900 & 61,600 \\
\hline $1934-1938$ & $1,188,800$ & 4,100 & 874,400 & 223,500 & 180,600 & 42,900 & 74,000 & 12,800 & 86,800 \\
\hline \multicolumn{10}{|l|}{ Annual: } \\
\hline 1927 & $1,443,000$ & 2,400 & $1,140,000$ & 220,100 & 12,600 & 199,000 & 69,000 & 11,500 & 80,500 \\
\hline 1928 & $1,346,000 *$ & 1,600 & $1,044,000$ & 230,700 & 28,000 & 191,000 & 59,000 & 10,700 & 69,700 \\
\hline 1929 & $1,098,000$ & 2,300 & 860,000 & 152,100 & 24,000 & 118,900 & 72,100 & 11,500 & 83,600 \\
\hline 1930 & $988,000^{*}$ & 1,300 & 767,000 & 148,600 & 22,000 & 110,800 & 59,800 & 11,300 & 71,100 \\
\hline 1931 & 775,000 & 300 & 676,000 & 48,300 & 4,600 & 35,200 & 40,300 & 10,100 & 50,400 \\
\hline 1932 & $1,200,000^{*}$ & 500 & $1,048,000$ & 88,100 & 12,000 & 74,000 & 49,000 & 14,400 & 63,400 \\
\hline 1933 & 970,000 & 970 & 780,000 & 136,000 & 80,000 & 56,000 & 40,000 & 13,030 & 53,030 \\
\hline 1934 & 928,000 & 2,200 & 684,000 & 165,200 & 140,000 & 25,200 & 61,700 & 14,900 & 76,600 \\
\hline 1935 & $1,248,000$ & 2,400 & 812,000 & 356,500 & 300,000 & 56,500 & 65,300 & 11,800 & 77,100 \\
\hline 1936 & 918,000 & 3,200 & 728,000 & 102,700 & 67,000 & 35,700 & 72,100 & 12,000 & 84,100 \\
\hline 1937 & $1,407,000$ & 7,500 & 988,000 & 312,100 & 254,000 & 58,100 & 87,000 & 12,400 & 99,400 \\
\hline 1938 & $1,443,000$ & 5,000 & $1,160,000$ & 180,900 & 142,000 & 38,900 & 84,100 & 13,000 & 97,100 \\
\hline 1939 & $1,269,000$ & 12,000 & 980,000 & 184,600 & 140,000 & 44,600 & 74,400 & 18,000 & 92,400 \\
\hline 1940 & $1,213,000$ & 11,300 & 684,000 & 417,500 & 358,000 & 59,500 & 81,300 & 18,900 & 100,200 \\
\hline 1941 Ф & $1,510,000$ & 12,000 & 836,000 & 548,000 & 496,000 & 52,000 & 100,000 & 14,000 & 114,000 \\
\hline
\end{tabular}

* The chief varieties included are Thompson Seedless, Muscats, Sultanas, and currants. Excludes unharvested tonnages as follows: $1928,60,000 ; 1930,319,000$; and $1932,21,000$.

+ In addition to total fresh grapes used for juice, col. 4, roughly 36,000 fresh tons a year of dried raisins were used for alcoholic beverages, 1927-1932. Wuch fewer raisins were probably so used since repeal.

F Includes small amounts used in the state for home wine making in 1927-1932 (col. 4 minus cols. 5 and 6 ).

$\oint$ Interstate juice shipments as shown are largely Muscats, of which a few are eaten fresh.

$q$ Unofficial revisions of production and utilization involving data on the commercial crush and the dried raisin pack released by the Wine Institute and the Dried Fruit Association in March, 1942.

Sources of data: Compiled by S. W. Shear, Giannini Foundation of Agricultural Economics, University of California.

Data largely based on reports of California Crop Reporting Service and of Federal-State Market News Service, except

estimates of fresh juice and table stock in all years, and of commercial crush in 1927-1929, were made by

S. W. Shear. 


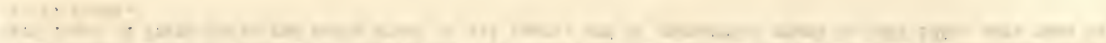

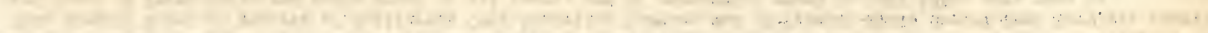

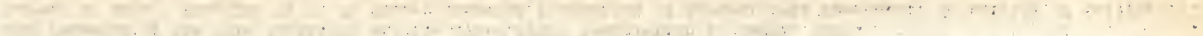

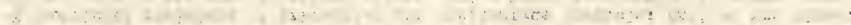

$\therefore$.

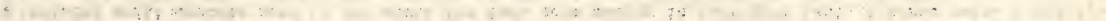

-

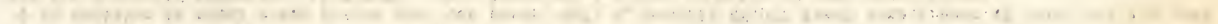

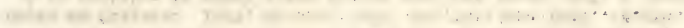

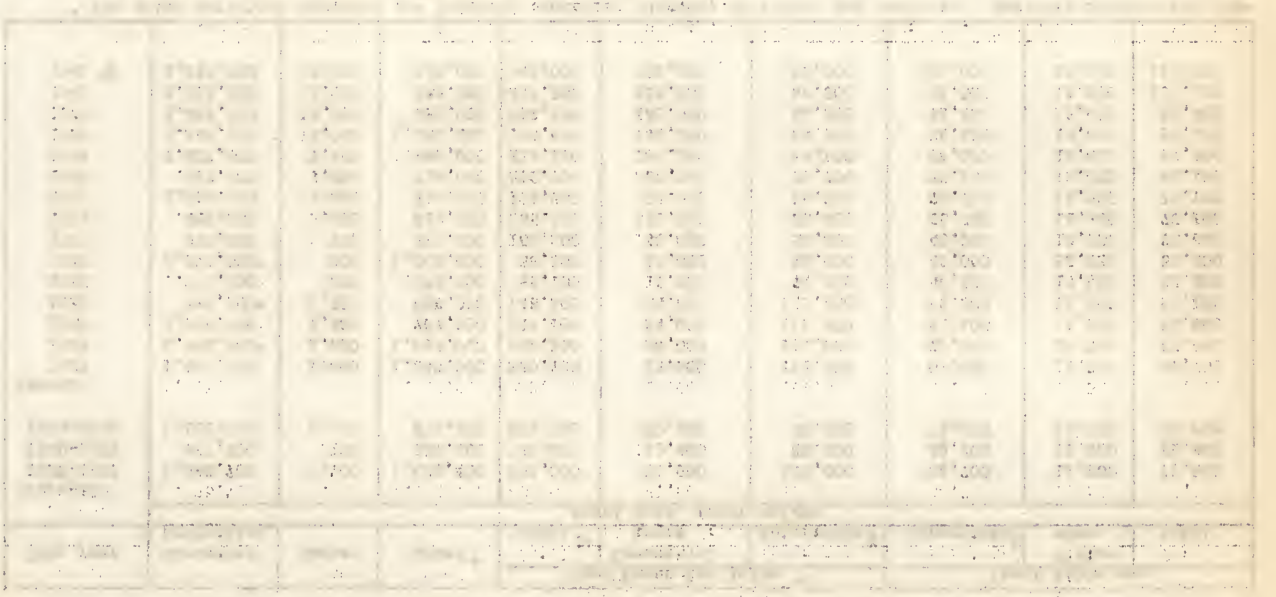

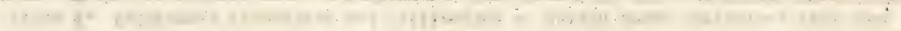


GRAPES

January, 1942

Table 8. California Production and Utilization of Table Grape Varieties*, 1927-1941

\begin{tabular}{|c|c|c|c|c|c|c|c|c|}
\hline \multirow[b]{2}{*}{ Crop year } & \multirow[b]{2}{*}{$\begin{array}{l}\text { Harvested } \\
\text { production }\end{array}$} & \multirow[b]{2}{*}{ Dried ${ }^{\dagger}$} & \multicolumn{3}{|c|}{ Used fresh for juice } & \multicolumn{3}{|c|}{ Fresh table use } \\
\hline & & & Totel & $\begin{array}{c}\text { Commercial } \\
\text { crush }\end{array}$ & Interstate $\$$ & Interstate & $\begin{array}{l}\text { Within } \\
\text { state }\end{array}$ & Total \\
\hline \multirow[b]{3}{*}{ Averages: } & 1 & 2 & 3 & 4 & 5 & 6 & 7 & 8 \\
\hline & \multicolumn{8}{|c|}{ Short tons, fresh weight } \\
\hline & & & & & & & & \\
\hline $1927-1929$ & 368,800 & 19,000 & 58,200 & 30,100 & 28,100 & 268,200 & 23,400 & 291,600 \\
\hline $1930-1932$ & 250,700 & 11,300 & 40,300 & 19,900 & 20,400 & 174,200 & 24,900 & 199,100 \\
\hline $1934-1938$ & 371,600 & 6,200 & 166,000 & 160,900 & 5,100 & 168,000 & 31,400 & 199,400 \\
\hline \multicolumn{9}{|l|}{ Annual: } \\
\hline 1927 & $391,400^{*}$ & 16,000 & 72,800 & 47,400 & 25,400 & 278,600 & 24,000 & 302,600 \\
\hline 1928 & $403,000^{*}$ & 16,000 & 51,300 & 27,800 & 23,500 & 313,500 & 22,200 & 335,700 \\
\hline 1929 & 312,000 & 25,000 & 50,400 & 15,000 & 35,400 & 212,600 & 24,000 & 236,600 \\
\hline 1930 & $314,000^{*}$ & 8,000 & 60,700 & 28,700 & 32,000 & 221,700 & 23,600 & 245,300 \\
\hline 1931 & 229,000 & 14,000 & 30,000 & 4,000 & 26,000 & 164,000 & 21,000 & 185,000 \\
\hline 1932 & $209,000^{\star}$ & 12,000 & 30,000 & 27,000 & 3,000 & 137,000 & 30,000 & 167,000 \\
\hline 1933 & $267,000^{*}$ & 12,200 & 106,600 & 104,000 & 2,600 & 123,700 & 24,500 & 148,200 \\
\hline 1934 & 296,000 & 9,200 & 100,000 & 89,000 & 11,000 & 159,200 & 27,600 & 186,800 \\
\hline 1935 & 375,000 & 3,600 & 196,000 & 195,000 & 1,000 & 144,800 & 30,600 & 175,400 \\
\hline 1936 & 324,000 & 5,400 & 112,800 & 105,000 & 7,800 & 175,300 & 30,500 & 205,800 \\
\hline 1937 & 416,000 & 5,800 & 193,200 & 191,000 & 2,200 & 183,300 & 33,700 & 217,000 \\
\hline 1938 & 447,000 & 6,800 & 228,100 & 224,600 & 3,500 & 177,300 & 34,800 & 212,100 \\
\hline 1939 & 390,000 & 8,000 & 181,800 & 177,000 & 4,800 & 166,000 & 34,200 & 200,200 \\
\hline 1940 & 430,000 & 4,000 & 199,300 & 197,000 & 2,300 & 189,800 & 36,900 & 226,700 \\
\hline $1941 \oint$ & 480,000 & 4,000 & 246,300 & 244,000 & 2,300 & 197,700 & 32,000 & 229,700 \\
\hline
\end{tabular}

- Chief varieties included in table grape production are Tokay, Malaga, Emperor, Red Malaga, Cornichon, Almeria, Ribier and Concord. Data given exclude unharvested tonnages: 1927, 98,600 (estimate by S. W. Shear); $1928,75,000 ; 1930,74,000 ; 1932,108,000$; and $1933,3,000$.

+ Dried table grape varieties are presumably used for juice purposes eventually.

F Probably very few table grape varieties are used for home wine making in California and all intrastate use as given in col. 7 is assumed as used for fresh table purposes.

Unofficial revisions involving data on the commercial crush released by the Wine Institute in March, 1942.

Sources of data: Compiled by S. W. Shear, Giannini Foundation, University of California. Data based largely on California Crop Reports and Federal-State Market News Reports, except estimates of fresh juice and table stock in all years and of commercial crush in 1927-1929 by S. N. Shear. 


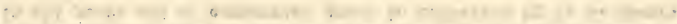

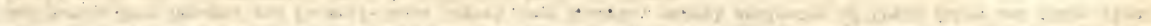

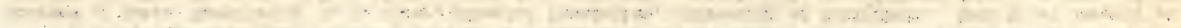

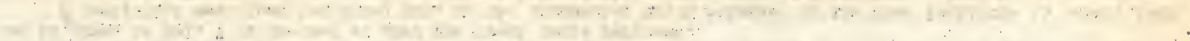

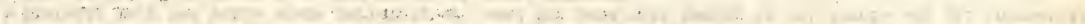

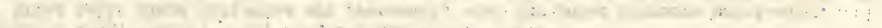

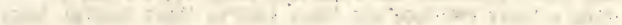

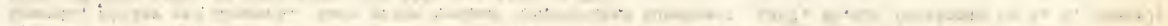

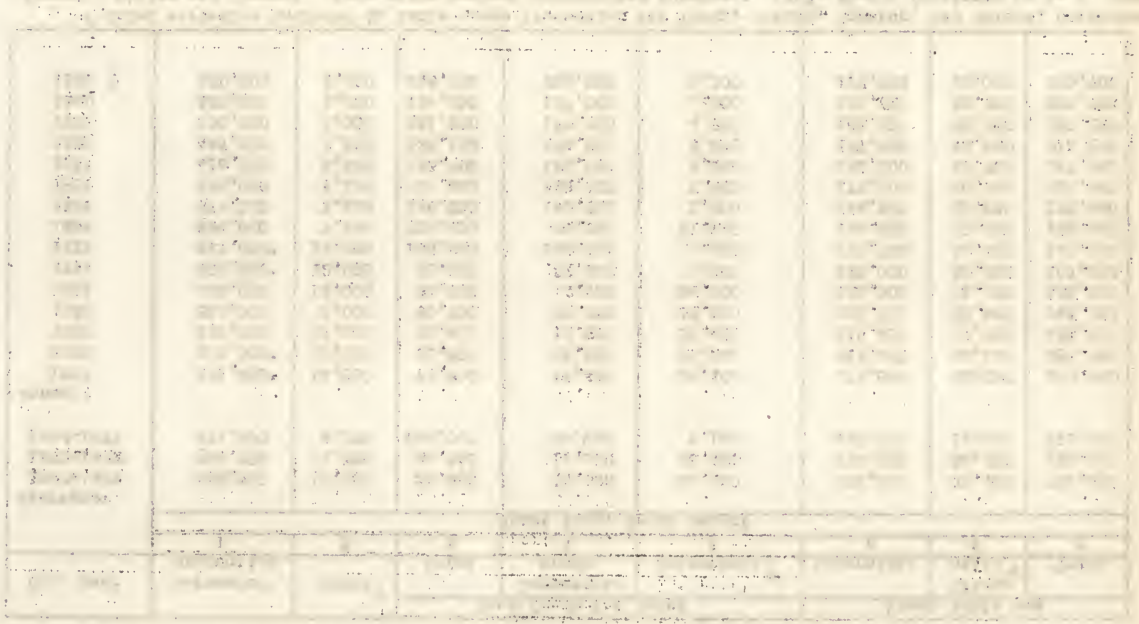

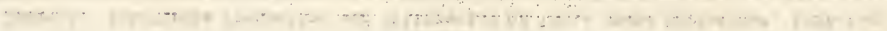


GRAPH;

January, 1942

Table 9

California Production and Utilization of wine Grape varieties*, 1927-1941

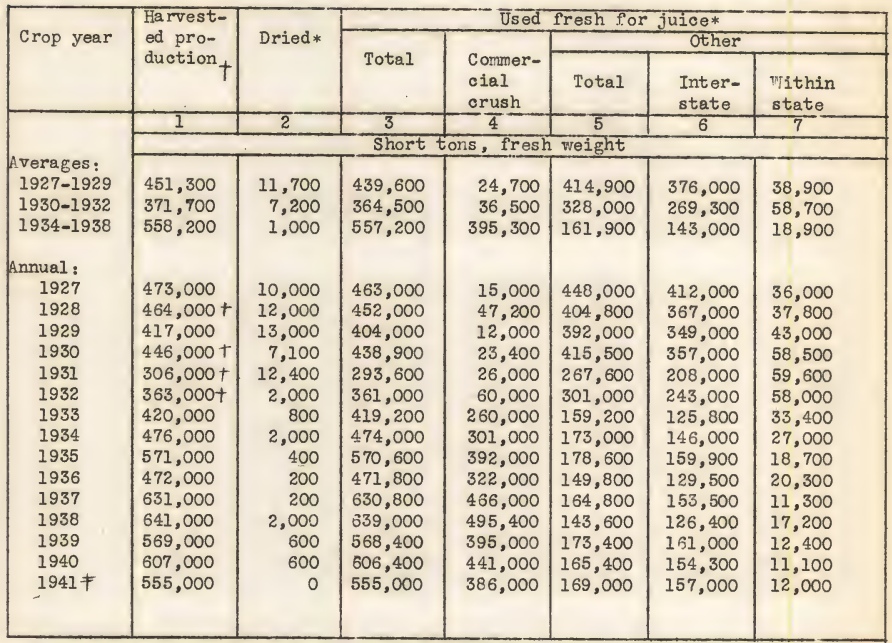

* The chief varieties included in wine grape varieties by the Crop Reporting Service in accordance with the most usual use of each variety are Zinfandel, Alicante Bouschet, Carignane, Detit Sirah, Ission, inataro, Grenache, Golden Chasselas, Burger, Colombar, and Franken Riesling. Presumably all Califormia grapes classified as strictly wine varieties,. including those dried, are crushed commercially or otherwise used for wine brandy and other juice purposes and practically none consumed for fresh table use.

$\dagger$ Excludes unharvested tonnages: 1928, 18,000; 1930, 40,000; 1931, 10,000; and $1932,25,000$.

F Unofficial revisions of production and utilization involving data on the commercial crush released by the "ine Institute in iarch, 1942.

Sources of data:

Compiled by S. W. Shear, Giannini Foundation of Agricultural Economics, University of California. Data are largely based on reports of the California Crop Reporting Service and of the San Francisco Federal-State l/arket News Service, except commeroial crush 1927-1929 estimated roughly by S. W. Shear. 
$\therefore \cdot \cdots+\cdots$

2.

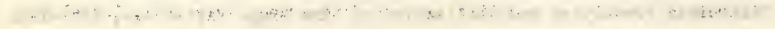

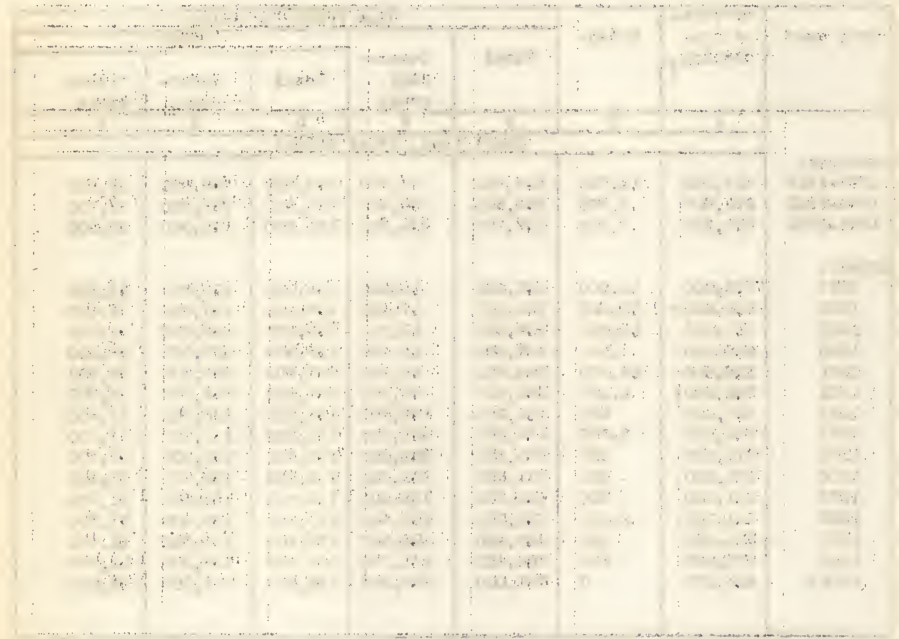

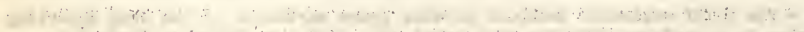

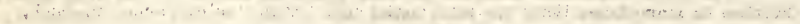

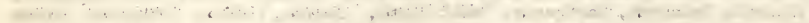

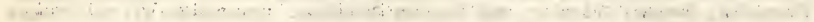

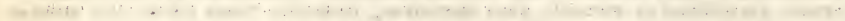

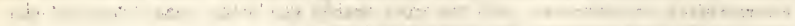

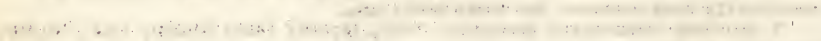
,

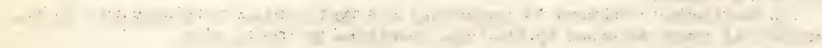

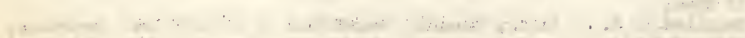

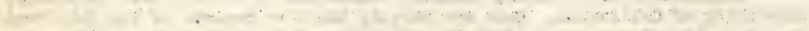

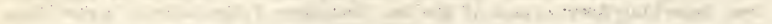

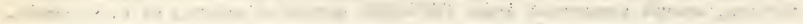




\section{GRAPES}

Table 10

California Interstate Grape Shipments* and Delivered-Auction Prices by Classes, 1921-1941

\begin{tabular}{|c|c|c|c|c|c|c|}
\hline \multirow{2}{*}{$\begin{array}{l}\text { Crop } \\
\text { year }\end{array}$} & \multicolumn{2}{|c|}{$\begin{array}{l}\text { Table varieties } t \\
\text { and Thompson Seedless }\end{array}$} & \multicolumn{2}{|c|}{ Wine varietiest } & \multicolumn{2}{|c|}{ Muscats } \\
\hline & Shipments & $\begin{array}{l}\text { Price per } \\
\text { package }\end{array}$ & Shipments & $\begin{array}{c}\text { Price per } \\
\text { package }\end{array}$ & Shipments & $\begin{array}{c}\text { Frice per } \\
\text { package }\end{array}$ \\
\hline & 1 & 2 & 3 & 4 & 5 & 6 \\
\hline & Cars & Dollars & Cars & Dollars & Cars & Dollars \\
\hline 1921 & 13,500 & 2.16 & 14,000 & 2.38 & 2,700 & 1.50 \\
\hline 1922 & 17,100 & 1.73 & 18,600 & 2.05 & 5,700 & 1.40 \\
\hline 1923 & 24,300 & 1.55 & 20,000 & 1.76 & 8,000 & 1.08 \\
\hline 1924 & 27,300 & 1.38 & 17,200 & 1.96 & 10,300 & 1.12 \\
\hline 1925 & $29,900 \neq$ & 1.17 & $23,800 \neq$ & 1.66 & $17,500 \neq$ & 0.97 \\
\hline 1926 & $27,100 \neq$ & 1.26 & $24,300 \neq$ & 1.50 & $9,400 \neq$ & 1.02 \\
\hline 1927 & 27,711 & 1.30 & $29,775^{\top}$ & 1.44 & 14,813 & 1.02 \\
\hline 1928 & 28,267 & 1.19 & 26,459 & 1.11 & 13,759 & 0.81 \\
\hline 1929 & 22,989 & 1.44 & 24,813 & 1.24 & 8,336 & 1.08 \\
\hline 1930 & 25,196 & 1.22 & 27,468 & 1.07 & 8,221 & 1.06 \\
\hline 1931 & 18,045 & 1.44 & 15,917 & 1.13 & 2,727 & 1.18 \\
\hline 1932 & 15,420 & 1.13 & 17,348 & 0.87 & 6,212 & 0.76 \\
\hline 1933 & 13,830 & 1.29 & 8,968 & 1.06 & 3,934 & 0.99 \\
\hline 1934 & 15,867 & 1.43 & 10,344 & 1.08 & 2,000 & 1.11 \\
\hline 1935 & 14,504 & 1.27 & 11,237 & 1.01 & 4,136 & 0.94 \\
\hline 1936 & 17,612 & 1.47 & 9,030 & 1.27 & 2,675 & 1.16 \\
\hline 1937 & 18,384 & 1.36 & 10,772 & 1.13 & 4,414 & 0.99 \\
\hline 1938 & 17,170 & 1.30 & 8,878 & 1.16 & 3,308 & 1.15 \\
\hline 1939 & 16,845 & 1.28 & 10,111 & 1.16 & 2,960 & 1.15 \\
\hline 1940 & 19,477 & 1.31 & 9,639 & 1.27 & 3,732 & 1.13 \\
\hline 1941 & 19,019 & 1.669 & 9,803 & 1.478 & 3,249 & $1.53 \oint$ \\
\hline
\end{tabular}

* Rail shipments of other states omitted because a large proportion is now shipped by truck (see production, other states, table 2, col. 2.) + Classified according to chief purpose for which shipments are used in eastern markets. Fresh interstate i uscat shipments are used chiefly for juice but include a few for table use.

F Shipments for 1925 and $1926^{\circ}$ are subject to revision. 6 Preliminary

Sources of data: Compiled by S.W. Shear, Giannini Foundation of Agricultural Economios, University of California.

Cols. 1,3, and 5: Include some estimates by $\mathrm{S} . \mathrm{W}$. Shear in earlier years but 1927-1941 data from Federal-state larket liews Service reports.

Cols. 2, 4, and 6: Delivered auction prices since 1924 are oompiled from Federal-State rarket News reports and are the weighted average prices of eleven miarkets (except table grapes 1921-1923 for 1!ew York only) for the principal varieties included in each class. Table varieties included in col. 2 are Cornichon, Kalaga, Red "alaga, Tokay, Emperor, and Thompson Seedless. 


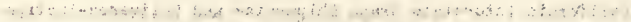

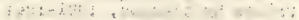

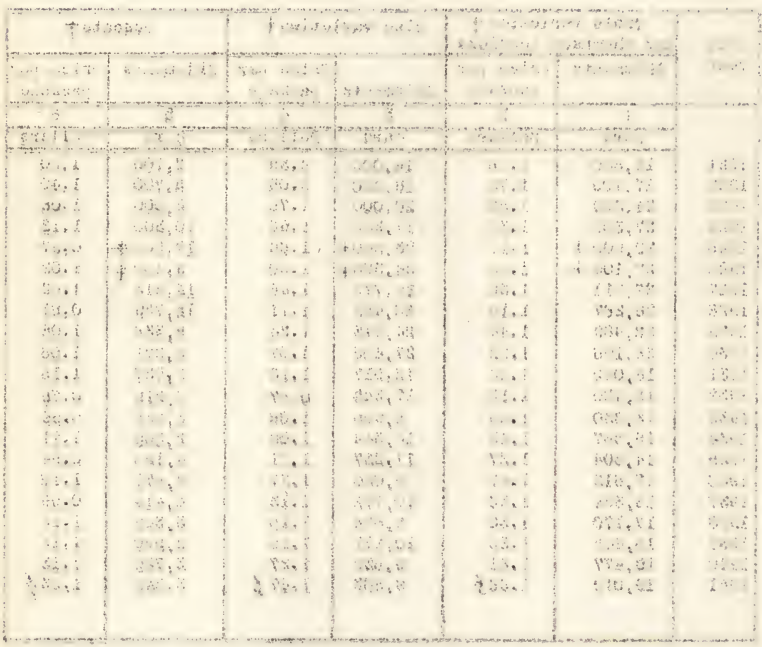

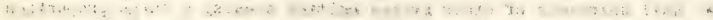

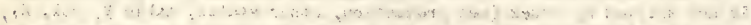

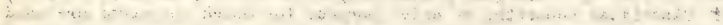

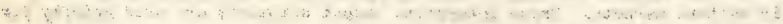

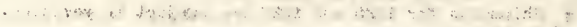

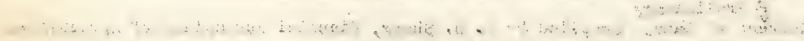

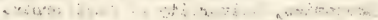

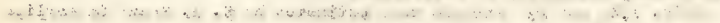

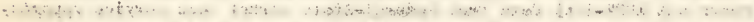

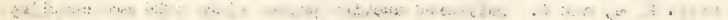

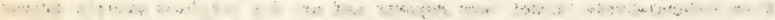

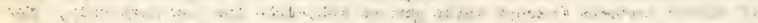

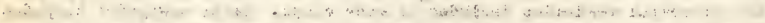

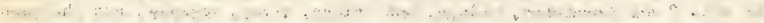


GRAPES

Table 11

January, 1942

United States Domestic Exports of Fresh Grapes by Countries of Destination, Years Beginning July 1, 1929-1940

\begin{tabular}{|c|c|c|c|c|c|c|c|c|c|c|c|c|}
\hline $\begin{array}{l}\text { Country of } \\
\text { destin-tion }\end{array}$ & $|19<9-30|$ & $\mid 1930-31$ & $\mid 1931-32$ & $1932-33$ & $\mid 1933-34$ & $1934-35$ & $|1935-36|$ & $1936-37$ & $1937-38$ & $1938-39$ & $1939-40$ & $1940-41$ \\
\hline & & & & hort $t$ & ons, net & t declere & ed fresh & weight & & & & \\
\hline Total exports & 23,079 & 24,900 & 13,807 & 14,676 & 13,344 & 17,856 & 18,676 & 23,058 & 35,014 & 39,928 & 29,980 & $30,330 *$ \\
\hline Europe, total & 129 & 1,313 & 798 & 2,504 & 1,687 & 2,632 & 3,502 & 4,984 & 12,304 & 18,066 & 4,172 & 108 \\
\hline United Kingdom & 68 & 1,096 & 609 & 2,325 & 1,548 & 2,339 & 3,184 & 4,210 & 10,643 & 15,205 & 2,661 & 86 \\
\hline Other & 61 & 217 & -189 & 179 & 139 & 293 & 318 & 774 & 1,661 & 2,861 & 1,511 & 22 \\
\hline France & 0 & 0) & 1 & 0 & 0 & 39 & 1 & 0 & 아 & 31 & 0 & 0 \\
\hline Netherlands & 9 & 11 & 이 & 50 & 이 & 이 & 23 & 3 & 64 & 87 & 0 & 0 \\
\hline Belgium & 0 & 0 & 3 & 1 & 10 & 이 & 이 & 이 & $t_{--1}$ & 이 & 0 & 0 \\
\hline Norway & 9 & 2 & 3 & 25 & 37 & 103 & 22 & 147 & 465 & 722 & 762 & 0 \\
\hline Sweden & 6 & 212 & 118 & 96 & 57 & 63 & 190 & 414 & 671 & 1,248 & 737 & 0 \\
\hline Denmark & 1 & 1 & 5 & 6 & 8 & -- & 이 & 12 & -1 & 이 & 0 & 0 \\
\hline Germany & 36 & 1 & 59 & 1 & 17 & 40 & 13 & - & 83 & 93 & 0 & 0 \\
\hline Finlend & 이 & 0 & 이 & 0 & 10 & 20 & 69 & 182 & 378 & 680 & 8 & 0 \\
\hline Other & -- & 0 & -- & - & 이 & 28 & - & 16 & 이 & 이 & 4 & 22 \\
\hline Except Europe, & 22,950 & 23,587 & 13,009 & 12,172 & 11,657 & 15,224 & 15,174 & 18,074 & 22,710 & 21,862 & 25,808 & 30,222 \\
\hline Canada & 16,488 & 17,039 & 9,228 & 8,447 & 7,948 & 9,154 & 9,550 & 12,377 & 14,848 & 13,923 & 15,567 & 20,525 \\
\hline Other & 6,462 & 6,548 & 3,781 & 3,725 & 3,709 & 6,070 & 5,624 & 5,697 & 7,862 & 7,939 & 10,241 & 9,697 \\
\hline Mexico & 1,522 & 1,521 & 198 & 409 & 391 & 548 & 541 & 738 & 1,666 & 1,511 & 4,046 & 3,826 \\
\hline Cuba & 1,923 & 1,821 & 804 & 755 & 396 & 934 & 881 & 842 & 1,177 & 1,135 & 1,025 & 1,101 \\
\hline Philippine Is. & 823 & 967 & 830 & 859 & 805 & 1,162 & 765 & 698 & 906 & 1,267 & i, 134 & 796 \\
\hline Brazil & 6 & 48 & 68 & 46 & 29 & 91 & 142 & 592 & 922 & 766 & 992 & 1,567 \\
\hline British lialaya & 227 & 142 & 154 & 109 & 224 & 608 & 603 & 410 & 597 & 504 & 462 & 450 \\
\hline New Zealand & 341 & 276 & 233 & 120 & 170 & 249 & 272 & 357 & 412 & 552 & 354 & -- \\
\hline Others & 1,620 & 1,773 & 1,494 & 1,427 & 1,694 & 2,478 & 2,4201 & 2,060 & 2,182 & 2,204 & 2,228 & 1,957 \\
\hline
\end{tabular}

* Nine months only, July 1940-inrch 1941 as data by countries of destin.tion not available for later months. Total exports for July - June vere 30,523 tons.

+ Dashes indicete less than 0.5 of a ton.

Sources of dota: Compiled by S. . Shecr, Giannini Foundation of igricultaral Economics, University of California, largely from U. S. Dept. wgr., Office of Foreign mgricultural Rel-tions or Bur. of Aar. Econ., Statisticsl Report. of the Deciduous Fruit xport Season, 1935-5. p. 4-24, December 1936 and 1939-40, p. 40, January 1941. 


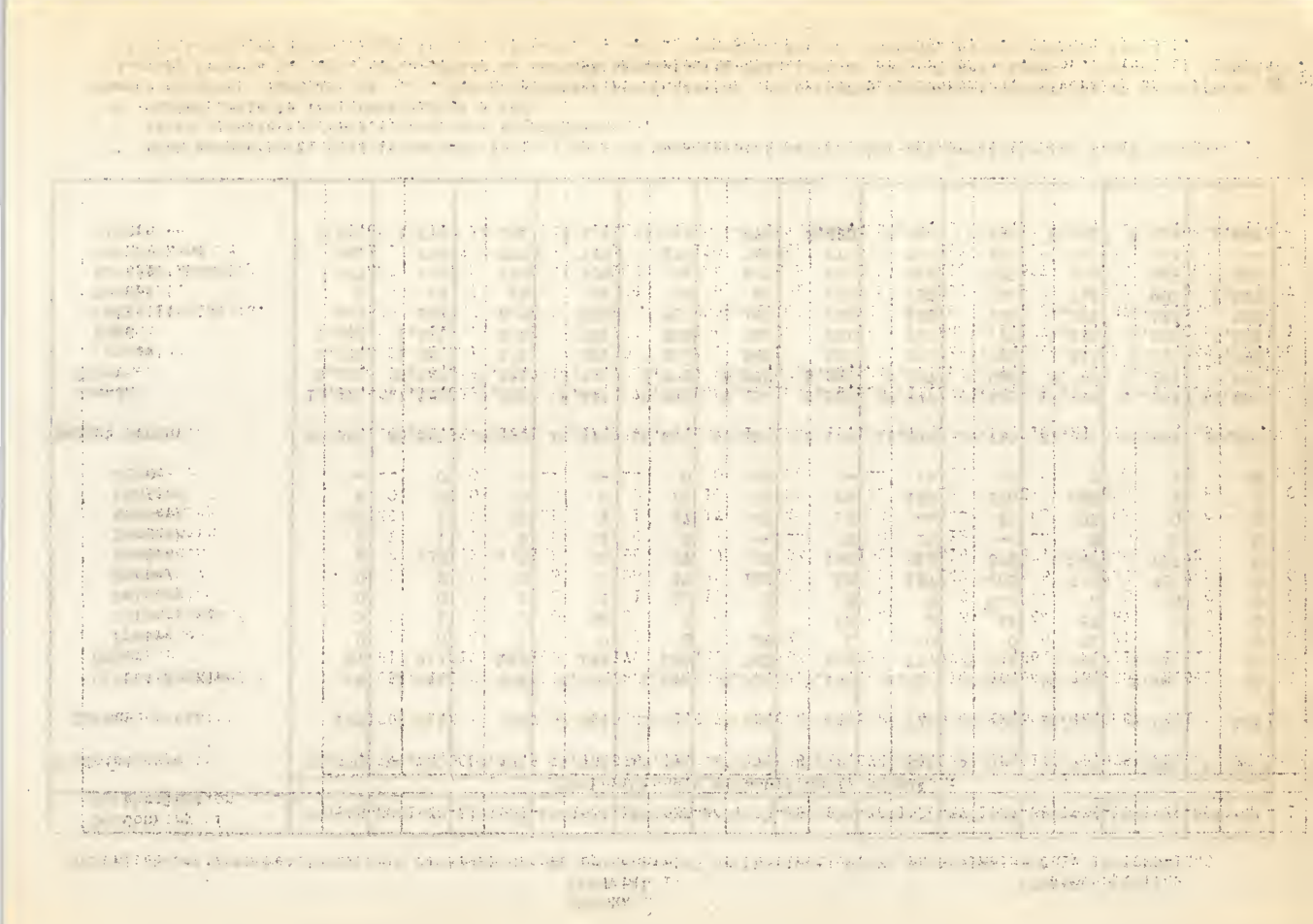




\section{GRAPES}

Table 12

Prices to Growers for California Natural iuscat and Thompson Seedless Raisins 1909-1941

\begin{tabular}{|c|c|c|c|c|c|c|c|c|c|}
\hline \multirow[t]{2}{*}{$\begin{array}{l}\text { Crop } \\
\text { year }\end{array}$} & \multicolumn{2}{|c|}{$\begin{array}{l}\text { Price per } \\
\text { dry ton }\end{array}$} & \multicolumn{2}{|c|}{$\begin{array}{l}\text { Equivalent price } \\
\text { per fresh ton at } \\
3.75 \text { to } 1 \text { drying } \\
\text { ratio }\end{array}$} & \multirow[t]{2}{*}{$\begin{array}{l}\text { Crop } \\
\text { year }\end{array}$} & \multicolumn{2}{|c|}{$\begin{array}{l}\text { Price per } \\
\text { dry ton }\end{array}$} & \multicolumn{2}{|c|}{$\begin{array}{l}\text { Equivalent price } \\
\text { per fresh ton at } \\
3.75 \text { to } 1 \text { drying } \\
\text { ratio }\end{array}$} \\
\hline & Rusoats & $\begin{array}{l}\text { Thompson } \\
\text { Seedless }\end{array}$ & Musoats & $\begin{array}{l}\text { Thompson } \\
\text { Seedless }\end{array}$ & & Muscats & $\begin{array}{l}\text { Thompson } \\
\text { Seedless }\end{array}$ & Muscats & $\begin{array}{l}\text { Thompson } \\
\text { Seedless }\end{array}$ \\
\hline & 1 & 2 & 3 & 4 & & 1 & 2 & 3 & 4 \\
\hline & \multicolumn{4}{|c|}{ Dollars } & & \multicolumn{4}{|c|}{ Dollars } \\
\hline 1909 & 33 & 43 & 9 & 11 & 1926 & 66 & 62 & 18 & 17 \\
\hline 1910 & 55 & 60 & 15 & 16 & 1927 & 50 & 57 & 13 & 15 \\
\hline 1911 & 75 & 108 & 20 & 29 & 1928 & 43 & 43 & 11 & 11 \\
\hline 1912 & 62 & 68 & 17 & 18 & 1929 & 62 & 08 & 17 & 18 \\
\hline 1913 & 70 & 79 & 19 & 21 & 1930 & 49 & 60 & 13 & 10 \\
\hline 1914 & 67 & 93 & 18 & 25 & 1931 & 60 & 70 & 16 & 19 \\
\hline 1915 & 73 & 100 & 19 & 27 & 1932 & 21 & 41 & 6 & 11 \\
\hline 1916 & 85 & 132 & 23 & 35 & 1933 & 53 & 56 & 14 & 15 \\
\hline 1917 & 97 & 138 & 26 & 37 & 1934 & 56 & 61 & 15 & 16 \\
\hline 1918 & 106 & 138 & 28 & 37 & 1935 & 54 & 56 & 14 & 15 \\
\hline 1919 & 208 & 240 & 55 & 64 & 1930 & 70 & 35 & 19 & 17 \\
\hline 1920 & 223 & 296 & 59 & 79 & 1937 & 64 & 61 & 17 & 16 \\
\hline 1921 & 146 & 168 & 39 & 45 & 1938 & 50 & 50 & 13 & 13 \\
\hline 192.2 & 54 & 73 & 14 & 19 & 1939 & 45 & 45 & 12 & 12 \\
\hline$\mp 323$ & 51 & 49 & 14 & 13 & 1940 & 57 & 55 & 15 & 15 \\
\hline 1924 & 61 & 62 & 16 & 17 & $1941=$ & 79 & 82 & 21 & 22 \\
\hline 1925 & 84 & 75 & 22 & 20 & & & & & \\
\hline & & & & & & & & & \\
\hline
\end{tabular}

* Preliminary.

\section{Sources of data:}

Compiled by S.... Shear, Giannini Foundation of E.Ericultural Economics, University of California.

Cols. 1 and 2: ieighted average prices of natural raisins for the regular trade (excluding sales of bleached and of surplus for distilling and other byproducts) paid growers by Sun-i aid 1913-1924 and by Sun-iaid and other packers for other years. Data for 1932 to date are estimates subject to minor revision based upon Raisin tarket Information Bulletins of the Federal-state ifarket jews service, Sacramento, California, and reliable confidential trade information.

Cols. 3 and 4: Computed from cols. 1 and 2 by dividing by 3.75 . 

GRAPES

Table 13

Shipments of California Raisins by Countries and F.0.B. Prices of Thompson Seedless Raisins, $1921-1940$

\begin{tabular}{|c|c|c|c|c|c|c|c|c|c|}
\hline \multirow{3}{*}{$\begin{array}{l}\text { Year } \\
\text { begin- } \\
\text { ning } \\
\text { Sept. } 1\end{array}$} & \multirow{3}{*}{$\begin{array}{l}\text { Grand } \\
\text { total }\end{array}$} & \multicolumn{3}{|c|}{ Domestic } & \multicolumn{4}{|c|}{ Exports } & \multirow{3}{*}{$\begin{array}{l}\text { F.o.b. price } \\
\text { Thompsons* } \\
\text { per net } \\
\text { packed pound }\end{array}$} \\
\hline & & \multirow{2}{*}{$\begin{array}{l}\text { Total United } \\
\text { States and } \\
\text { Canads }\end{array}$} & \multirow[b]{2}{*}{$\begin{array}{l}\text { United } \\
\text { States }\end{array}$} & \multirow[b]{2}{*}{ Canada } & \multicolumn{2}{|c|}{ Total } & \multirow[b]{2}{*}{$\begin{array}{l}\text { United } \\
\text { Kingdom }\end{array}$} & \multirow[b]{2}{*}{$\begin{array}{c}\text { Other } \\
\text { countries }\end{array}$} & \\
\hline & & & & & $\begin{array}{c}\text { Including } \\
\text { Canada }\end{array}$ & $\begin{array}{c}\text { Excluding } \\
\text { Canada }\end{array}$ & & & \\
\hline & \multicolumn{8}{|c|}{ Short tons, sweat box weight } & Cents \\
\hline 2921 & 155,000 & 139,700 & 125,200 & 14,500 & 29,800 & 15,300 & 11,000 & 4,300 & 14.0 \\
\hline 1922 & 190,000 & 153,500 & 135,000 & 18,500 & 55,000 & 36,500 & 20,400 & 16,100 & 10.5 \\
\hline 1923 & 195,000 & 168,400 & 149,400 & 19,000 & 45,600 & 26,600 & 8,300 & 18,300 & 7.3 \\
\hline 1924 & 220,000 & 187,600 & 167,600 & 20,000 & 52,400 & 32,400 & 14,800 & 17,600 & 7.4 \\
\hline 1925 & 240,000 & 185,300 & 168,000 & 17,300 & 72,000 & 54,700 & 23,700 & 31,000 & 7.3 \\
\hline 1926 & 245,000 & 182,400 & 162,300 & 20,100 & 82,700 & 62,600 & 28,500 & 34,100 & 6.8 \\
\hline 1927 & 285,000 & 199,600 & 178,000 & 21,600 & 107,000 & 85,400 & 37,700 & 47,700 & 5.6 \\
\hline 1928 & 290,000 & 193,400 & 171,000 & 22,400 & 119,000 & 96,600 & 37,500 & 52,100 & 4.4 \\
\hline 1929 & 215,000 & 162,900 & 148,600 & 14,300 & 66,400 & 52,100 & 19,100 & 33,000 & 4.9 \\
\hline 1930 & 215,000 & 160,100 & 148,200 & $11, .00$ & 66,800 & 54,900 & 21,700 & 33,200 & 4.7 \\
\hline 1931 & 185,000 & 131,000 & 124,100 & 6,900 & 60,900 & 54,000 & 24,100 & 29,900 & 5.1 \\
\hline 1932 & 220,000 & 160,700 & 155,300 & 5,400 & 64,700 & 59,300 & 26,500 & 32,800 & 3.3 \\
\hline 1933 & $190,000+$ & $143,300+$ & $137,500+$ & 5,800 & 52,500 & 46,700 & 17,800 & 28,900 & 4.2 \\
\hline 1934 & 190,000 & 145,800 & 141,800 & 4,000 & 48,200 & 44,200 & 18,800 & 25,400 & 4.3 \\
\hline 1935 & $220,000+$ & $163,500+$ & $159,100 \dagger$ & 4,400 & 60,900 & 56,500 & 28,500 & 28,000 & 4.1 \\
\hline 1936 & 200,000 & 144,800 & 140,900 & 3,900 & 59,100 & 55,200 & 26,000 & 29,200 & 4.8 \\
\hline 1937 & $225,000 \$$ & 151,200 o & $148,300 \delta$ & 2,900 & 76,700 & 73,800 & 32,000 & 41,800 & 4.0 \\
\hline 1938 & $220,000+6$ & $138,700+\S$ & $135,700+6$ & 3,000 & 84,300 & 81,300 & 34,100 & 47,200 & 3.7 \\
\hline $1939 \neq$ & $245,000 \neq \delta$ & $187,900 \neq \varnothing$ & $182,900 \neq \beta$ & 5,000 & 62,100 & 57,100 & 16,200 & 40,900 & 3.4 \\
\hline $1940 \neq$ & $230,000 \neq 6$ & $183,900 \neq\}$ & $180,700 \neq \$$ & $3,200 \neq$ & 49,300 & $46,100 \neq$ & & & 4.3 \\
\hline
\end{tabular}

* Prices 1921-1923 are for all raisins as prices of Thompson seedless sepsrately are not vailable.

+ In addition to sales to regular trade given, about 15,000 tons of suscats used by distillers in 1933 ; 5,000 tons of f-grades into by-products in 1935; and 52,000 tons from 1938 crop into stock feed or brandy in 1938 and 1939.

† Estimates are preliminary.

6 Data include tons shipped for relief: 1937, 15,000; 1938, approximately 10,000; 1939, 45,000; 1940, 32,000.

Sources of deta: Compiled by S. W. Shear, Giannini Foundation of Agricultural Bconomics, Monthly water and rail shipments from California plus estimated state consumption. Exports from Monthly Summary of Foreign Commerce Domestic shipments computed by suttraction. Prices $1921-29$ based on actual sales of packers; 1 ater years on California

Fruit Nevs nintations choice buik Thompsons, weighted by monthly shipments lagged. 


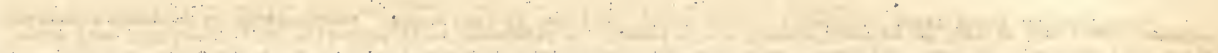

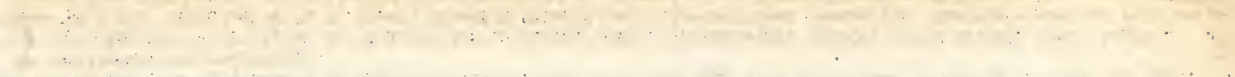

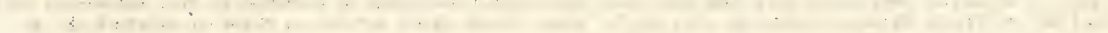

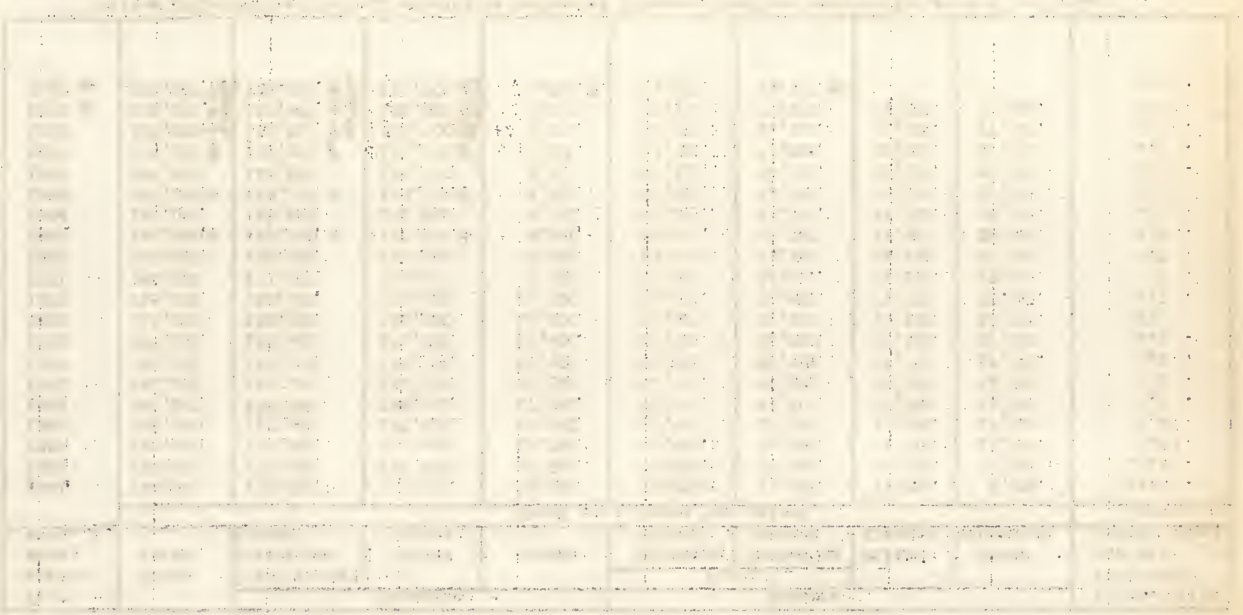

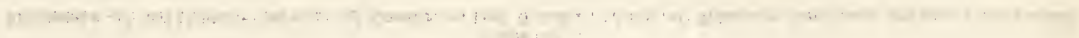


GRAPES

January, 1942

Table 14. World Raisin and Currant Production by Chief Countries, 1909-1941

\begin{tabular}{|c|c|c|c|c|c|c|c|c|c|c|}
\hline \multirow{3}{*}{$\begin{array}{c}\text { Year } \\
\text { harvested }\end{array}$} & \multicolumn{4}{|c|}{ Totals } & \multicolumn{6}{|c|}{ Raisins } \\
\hline & \multirow{2}{*}{$\begin{array}{l}\text { Raisins and } \\
\text { currants }\end{array}$} & \multirow[b]{2}{*}{ Currants } & \multicolumn{2}{|c|}{ Raisins } & \multirow{2}{*}{ Californis } & \multirow[b]{2}{*}{ Spain } & \multirow[b]{2}{*}{ Australia } & \multirow{2}{*}{$\begin{array}{c}\text { Smyrna } \\
\text { (Turkey) }\end{array}$} & \multirow{2}{*}{$\begin{array}{c}\text { Greece and } \\
\text { Crete } \neq\end{array}$} & \multirow{3}{*}{$\begin{array}{l}\text { Iran } \\
\text { (Persia) }\end{array}$} \\
\hline & & & World" & Foreign* & & & & & & \\
\hline \multirow{3}{*}{$\begin{array}{l}\text { Averages: } \\
1909-1913\end{array}$} & \multirow{2}{*}{\multicolumn{10}{|c|}{ Short tons, natural dry weight }} \\
\hline & & & & & & & & & & \\
\hline & 404,900 & 178,700 & 226,200 & 154,500 & 71,700 & 21,300 & 7,100 & 54,800 & 15,000 & 55,000 \\
\hline $1920-1924$ & 493,700 & 147,000 & 346,700 & 142,900 & 203,800 & 19,500 & 18,800 & 40,100 & 15,000 & 43,800 \\
\hline $1925-1929$ & 599,200 & 172,800 & 426,400 & 179,800 & 246,600 & 26,300 & 38,200 & 47,800 & 20,200 & 41,900 \\
\hline $1930-1934$ & 522,900 & 147,200 & 375,700 & 177,900 & 197,800 & 16,700 & 53,400 & 51,000 & 21,100 & 29,400 \\
\hline $1935-1939$ & 618,000 & 157,100 & 460,900 & 227,500 & 233,400 & 14,500 & 60,400 & 73,900 & 32,700 & 36,200 \\
\hline \multicolumn{11}{|l|}{ Annual: } \\
\hline 1925 & 538,600 & 180,100 & 358,500 & 158,500 & 200,000 & 33,600 & 28,600 & 32,500 & 18,500 & 40,000 \\
\hline 1926 & 625,100 & 197,000 & 428,100 & 156,100 & 272,000 & 25,900 & 25,100 & 39,200 & 16,500 & 44,000 \\
\hline 1927 & 648,800 & 154,500 & 494,300 & 209,300 & 285,000 & 25,800 & 49,000 & 56,000 & 24,600 & 48,500 \\
\hline 1928 & 612,500 & 168,000 & 444,500 & 183,500 & 261,000 & 25,200 & 28,800 & 49,300 & $25,600 \neq$ & 49,000 \\
\hline 1929 & 571,100 & 164,600 & 406,500 & 191,500 & 215,000 & 20,800 & 59,400 & 62,000 & $16,000 \neq$ & 28,000 \\
\hline 1930 & 524,700 & 168,600 & 356,100 & 164,100 & $-192,000$ & 17,600 & 59,900 & 38,900 & 15,000 & 28,000 \\
\hline 1931 & 414,100 & 93,100 & 321,000 & 152,000 & 169,000 & 16,300 & 36,800 & 29,700 & 15,000 & 48,000 \\
\hline 1932 & 605,100 & 161,300 & 443,800 & 181,800 & 262,000 & 21,200 & 45,800 & 71,600 & 22,000 & 15,000 \\
\hline 1933 & 535,600 & 143,400 & 392,200 & 197,200 & 195,000 & 11,500 & 68,600 & 60,700 & 28,000 & 20,000 \\
\hline 1934 & 535,300 & 169,800 & 365,500 & 194,500 & 171,000 & 16,800 & 56,000 & 54,000 & 25,500 & 36,000 \\
\hline 1935 & 632,200 & 191,500 & 440,700 & 237,700 & 203,000 & 21,000 & 51,400 & 87,000 & 35,500 & 35,000 \\
\hline 1936 & 553,100 & 146,800 & 406,300 & 224,300 & 182,000 & 17,500 & 56,700 & 71,200 & 29,500 & 40,000 \\
\hline 1937 & 586,800 & 152,300 & 434,500 & 187,500 & 247,000 & 11,000 & 59,400 & 48,000 & 27,000 & 32,000 \\
\hline 1938 & 693,200 & 147,000 & 546,200 & 256,200 & 290,000 & 13,000 & 79,600 & 82,000 & 34,000 & 36,000 \\
\hline 1939 & 624,800 & 147,800 & 477,000 & 232,000 & 245,000 & 10,200 & 55,000 & 81,000 & 37,500 & 38,000 \\
\hline 1940 & 527,600 & 161,400 & 366,200 & 195,200 & 171,000 & 9,400 & 78,400 & 33,000 & 25,300 & 38,000 \\
\hline $1941 \oint$ & & & & & 209,000 & & 69,900 & 55,000 & & 33,000 \\
\hline
\end{tabular}

* Totals include small quantities of raisins produced in the Union of South Africa.

$\dagger$ Greece and Australia produce nearly all the commercial currant crop of the world.

Fata for Greece and Crete exclude Rosakias since 1928 because unimportant.

$\phi$ Data are preliminary and unofficial.

Sources of data: Compiled by S. W. Shear, Giannini Foundation of Agricultural Economics, University of California.

California data from California Crop Reports exclude dried grapes other than raisin varieties. Foreign production : largely from reports of Office of Foreign Agricultural Relations, U. S. Dept. Agr. released currently by the FederalState Market News Service at Sacramento. 


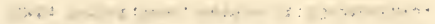

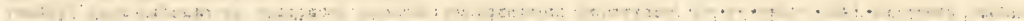

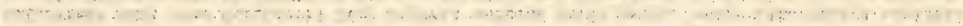

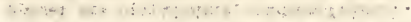

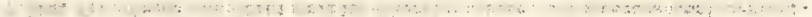

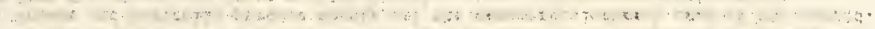

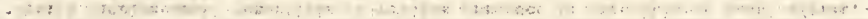

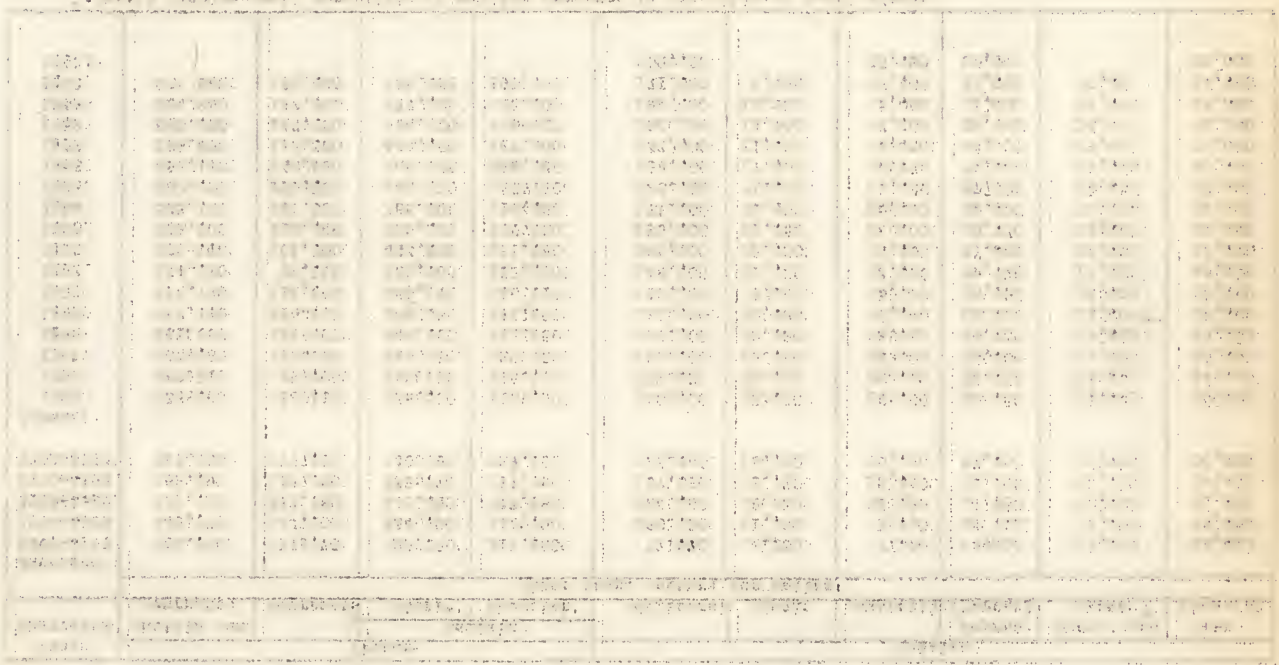

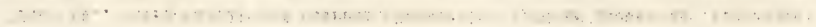




\section{GRAPES}

Table 15

Dried Currants: California Production, Acreage, Yield, Farm irice and Shipments, and United States Imports and Apparent Consumption, 1930-1941

\begin{tabular}{|c|c|c|c|c|c|c|c|c|c|c|c|}
\hline \multirow{3}{*}{$\begin{array}{c}\text { Yeer } \\
\text { beginning } \\
\text { Sept. } 1\end{array}$} & \multicolumn{9}{|c|}{ California } & \multicolumn{2}{|c|}{ United States } \\
\hline & \multirow{2}{*}{$\begin{array}{l}\text { Farm price } \\
\text { per } \\
\text { dry ton }\end{array}$} & \multicolumn{3}{|c|}{ Acreage } & \multirow[b]{2}{*}{$\begin{array}{c}\text { Yield } \\
\text { per } \\
\text { bearing } \\
\text { acre }\end{array}$} & \multirow[b]{2}{*}{ Production } & \multirow[b]{2}{*}{$\begin{array}{l}\text { Carry-in } \\
\text { Sept. } 1\end{array}$} & \multirow[b]{2}{*}{$\begin{array}{l}\text { Available } \\
\text { supply }\end{array}$} & \multirow[b]{2}{*}{ Shipments } & \multirow[b]{2}{*}{ Imports } & \multirow[b]{2}{*}{$\begin{array}{c}\text { spparent } \\
\text { consurption }\end{array}$} \\
\hline & & Total & $\begin{array}{c}\text { Non } \\
\text { bearing }\end{array}$ & Bearing & & & & & & & \\
\hline & 1 & 2 & 3 & 4 & 5 & 6 & 7 & 8 & 9 & 10 & 11 \\
\hline & Dollars & \multicolumn{3}{|c|}{ Acres } & \multicolumn{7}{|c|}{ Short tons, unprocessed dry weight } \\
\hline 1930 & 98 & 1,800 & 180 & 1,620 & 0.40 & 650 & 100 & 750 & 450 & 4,810 & 5,260 \\
\hline 1931 & 97 & 1,870 & 200 & 1,670 & 0.30 & 500 & 300 & 800 & 750 & 3,410 & 4,160 \\
\hline 1932 & 94 & 2,010 & 280 & 1,730 & 0.35 & 600 & 50 & 650 & 550 & 3,540 & 4,090 \\
\hline 1933 & 122 & 2,200 & 420 & 1,780 & 0.31 & 550 & 100 & 650 & 450 & 3,310 & 3,760 \\
\hline 1934 & 140 & 2,520 & 660 & 1,860 & 0.38 & 700 & 200 & 900 & 800 & 3,750 & 4,550 \\
\hline 1935 & 147 & 2,810 & 830 & 1.980 & 0.40 & 800 & 100 & 900 & 600 & 3,250 & 3,850 \\
\hline 1936 & 131 & 2,870 & 760 & 2,110 & 0.40 & 850 & 300 & 1,150 & 550 & 3,350 & 3,900 \\
\hline 1937 & 93 & 2,887 & 467 & 2,420 & 0.52 & 1,250 & 600 & 1,850 & 900 & 2,560 & 3,460 \\
\hline 1938 & 80 & 3,094 & 303 & 2,791 & 0.70 & 1,950 & 950 & 2,900 & 1,700 & 2,140 & 3,840 \\
\hline 1939 & 79 & 3,184 & 228 & 2,956 & 0.86 & 2,550 & 1,200 & 3,750 & 2,450 & 1,860 & 4,310 \\
\hline 1940 & 69 & 3,143 & 146 & 2,997 & 1.00 & 3,000 & 1,300 & 4,300 & & 580 & \\
\hline 1941 & $120^{*}$ & & & & & 3,600 & & & & & \\
\hline
\end{tabular}

* Rough preliminary estimetes.

Sources of data: Compiled by S. W. Shear, Giannini Foundation of Agricultural Economics, University of Celifornia. Cols. 1 and 6: Compiled jointly with R. E. Blair, California Cooperative Crop Reporting Service.

Cols. 2, 3, and 4: From Californis Cooperative Crop Reporting iervice.

Cols. 5, 8,, , and 11: Calculsted from data in other columns.

Col. 7: Based on stocks of packers hendling a lirge mejority of the currants.

Col. 10: Based on official releeses of the U. B. Bur. of For. End Dom. Com. 


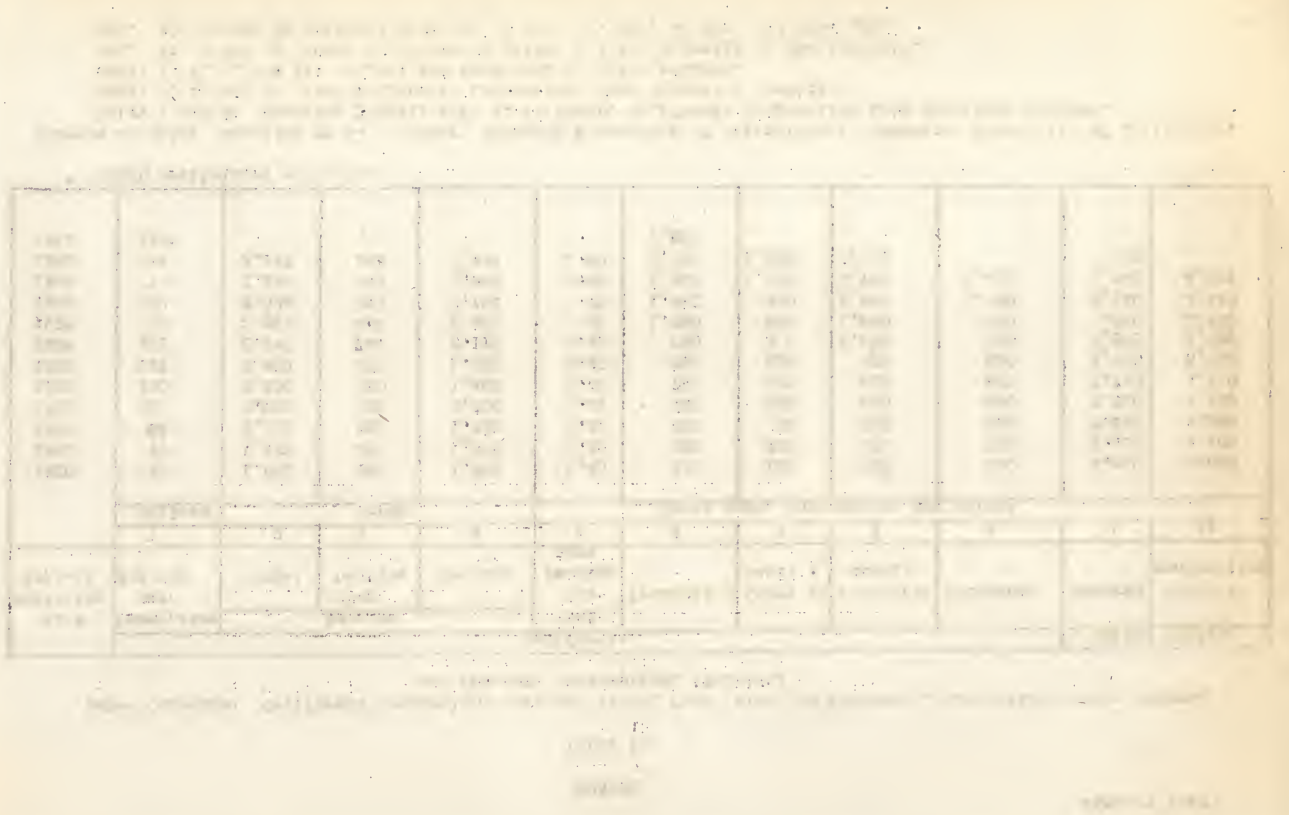


GRAPES

January, 1942

Table 16. United States Apparent Consumption of Still Wine, 1933-1941

\begin{tabular}{|c|c|c|c|c|c|c|c|c|c|c|c|c|}
\hline \multirow{3}{*}{$\begin{array}{l}\text { Year } \\
\text { begin- } \\
\text { ning } \\
\text { July } 1\end{array}$} & \multicolumn{6}{|c|}{ United States ${ }^{*}$} & \multicolumn{3}{|c|}{ In California } & \multicolumn{3}{|c|}{ In other states } \\
\hline & \multicolumn{2}{|c|}{ Total } & \multirow[b]{2}{*}{$\begin{array}{l}\text { Sweet, } \\
\text { over } 14 \\
\text { per cent } \\
\text { alcohol } \\
\text { commercial }\end{array}$} & \multicolumn{3}{|c|}{$\begin{array}{l}\text { Dry, not over } 14 \text { per } \\
\text { cent alcohol }\end{array}$} & \multirow[b]{2}{*}{ Total } & \multirow[b]{2}{*}{$\begin{array}{l}\text { Commer- } \\
\text { cial }\end{array}$} & \multirow[b]{2}{*}{$\begin{array}{l}\text { Home- } \\
\text { made }\end{array}$} & \multirow[b]{2}{*}{ Total } & \multirow[b]{2}{*}{$\begin{array}{c}\text { Commer } \\
\text { cial }\end{array}$} & \multirow[b]{2}{*}{$\begin{array}{l}\text { Home- } \\
\text { made }\end{array}$} \\
\hline & $\begin{array}{l}\text { Commer- } \\
\text { cial and } \\
\text { homemade }\end{array}$ & $\begin{array}{l}\text { Commer- } \\
\text { cial }\end{array}$ & & Totel & $\frac{\text { cent alco }}{\begin{array}{c}\text { Commer- } \\
\text { cial }\end{array}}$ & $\begin{array}{l}\text { Home- } \\
\text { made }\end{array}$ & & & & & & \\
\hline & 1 & 2 & 3 & 4 & 5 & 6 & 7 & 8 & 9 & 10 & 11 & 12 \\
\hline $\begin{array}{l}1933 \\
1934 \\
1935 \\
1936 \\
1937 \\
1938 \\
1939 \\
1940 \\
1941\end{array}$ & $\begin{array}{r}52,146 \\
70,916 \\
86,027 \\
95,338 \\
98,895 \\
99,753 \\
120,785 \\
125,305\end{array}$ & $\begin{array}{l}17,526 \\
37,856 \\
50,012 \\
65,503 \\
64,230 \\
70,533 \\
86,075 \\
90,535\end{array}$ & $\begin{array}{l}\text { Totel c } \\
10,973 \\
24,491 \\
32,958 \\
42,775 \\
41,350 \\
46,493 \\
59,453 \\
62,942\end{array}$ & $\begin{array}{l}\text { sumption } \\
41,173 \\
46,425 \\
53,069 \\
52,563 \\
57,545 \\
53,260 \\
61,332 \\
62,363\end{array}$ & $\begin{array}{r}\text { thousand } \\
6,553 \\
13,365 \\
17,054 \\
22,728 \\
22,880 \\
24,040 \\
26,622 \\
27,593\end{array}$ & $\begin{array}{l}\text { of gello } \\
\begin{array}{l}34,620 \\
33,060 \\
36,015 \\
29,835 \\
34,665 \\
29,220 \\
34,710 \\
34,770 \\
34,0956\end{array}\end{array}$ & $\begin{array}{l}13,010 \\
21,650 \\
23,957 \\
23,942 \\
21,523 \\
22,340 \\
22,608 \\
21,288\end{array}$ & $\begin{array}{l}8,000 T \\
17,600 T \\
21,152 \\
20,897 \\
19,828 \\
19,760 \\
20,748 \\
19,623\end{array}$ & $\begin{array}{l}5,010 \\
4,050 \\
2,805 \\
3,045 \\
1,695 \\
2,580 \\
1,860 \\
1,665 \\
1,8009\end{array}$ & $\begin{array}{r}39,136 \\
49,266 \\
62,070 \\
71,396 \\
77,372 \\
77,413 \\
98,177 \\
104,017\end{array}$ & $\begin{array}{r}9,526 \\
20,256 \\
28,860 \\
44,606 \\
44,402 \\
50,773 \\
65,327 \\
70,912\end{array}$ & $\begin{array}{l}29,610 \\
29,010 \\
33,210 \\
26,790 \\
32,970 \\
26,640 \\
32,850 \\
33,105 \\
32,2958\end{array}$ \\
\hline & & & & & Per-cap & a consur & on, ga & Ions & & & & \\
\hline $\begin{array}{l}1933 \\
1934 \\
1935 \\
1936 \\
1937 \\
1938 \\
1939 \\
1940 \\
1941\end{array}$ & $\begin{array}{l}.41 \\
.56 \\
.67 \\
.74 \\
.76 \\
.76 \\
.92 \\
.95\end{array}$ & $\begin{array}{l}.14 \\
.30 \\
.39 \\
.51 \\
.49 \\
.54 \\
.65 \\
.69\end{array}$ & $\begin{array}{l}.09 \\
.19 \\
.26 \\
.33 \\
.32 \\
.36 \\
.45 \\
.48\end{array}$ & $\begin{array}{l}.32 \\
.37 \\
.41 \\
.41 \\
.44 \\
.40 \\
.47 \\
.47\end{array}$ & $\begin{array}{l}.05 \\
.11 \\
.13 \\
.18 \\
.17 \\
.18 \\
.20 \\
.21\end{array}$ & $\begin{array}{l}.27 \\
.26 \\
.28 \\
.23 \\
.27 \\
.22 \\
.27 \\
.26 \\
.268\end{array}$ & $\begin{array}{l}2.20 \\
3.63 \\
3.97 \\
3.92 \\
3.39 \\
3.38 \\
3.30 \\
3.00\end{array}$ & $\begin{array}{l}1.35 \\
2.95 \\
3.51 \\
3.42 \\
3.12 \\
2.99 \\
3.03 \\
2.76\end{array}$ & $\begin{array}{l}.85 \\
.68 \\
.46 \\
.50 \\
.27 \\
.39 \\
.27 \\
.24 \\
.256\end{array}$ & $\begin{array}{l}.33 \\
.41 \\
.51 \\
.58 \\
.63 \\
.62 \\
.79 \\
.83\end{array}$ & $\begin{array}{l}.08 \\
.17 \\
.24 \\
.36 \\
.36 \\
.41 \\
.53 \\
.57\end{array}$ & $\begin{array}{l}.25 \\
.24 \\
.27 \\
.22 \\
.27 \\
.21 \\
.26 \\
.26 \\
.265\end{array}$ \\
\hline
\end{tabular}

Includes average imports, 1933-1940, of $2,936,000$ gals. or 3.1 per cent of consumption, about half dry and half sweet. tEstimates by S. W. Shear. $\$$ Preliminary estimates.

Sources of data: Compiled by S. W. Shear, Giannini Foundation of Agricultural Economics, University of California. Cols. 1, 2, 4, 7, 10-12: Calculated from data in other columns by addition or subtraction. Cols. 3 and 5 : Sum of domestic tax-paid withdrawals, Bureau Internal Revenue, and U. S. imports. Cols. 6 and 9: Homemade or basement wine estimated from California grapes so used, at 150 gallons per ton, but not for grapes produced outside of California. Very little homemade wine was consumed before prohibition. averaged about 0.5 gallons of which 0.2 was 


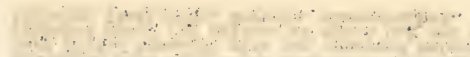

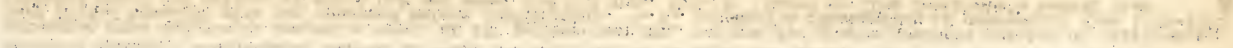

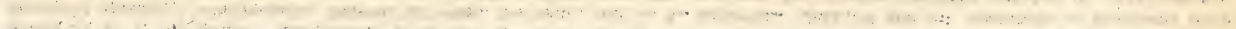

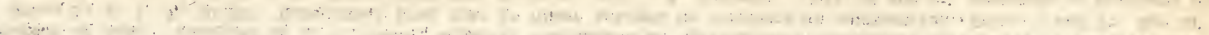

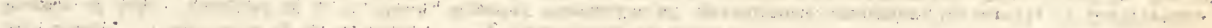

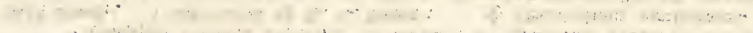

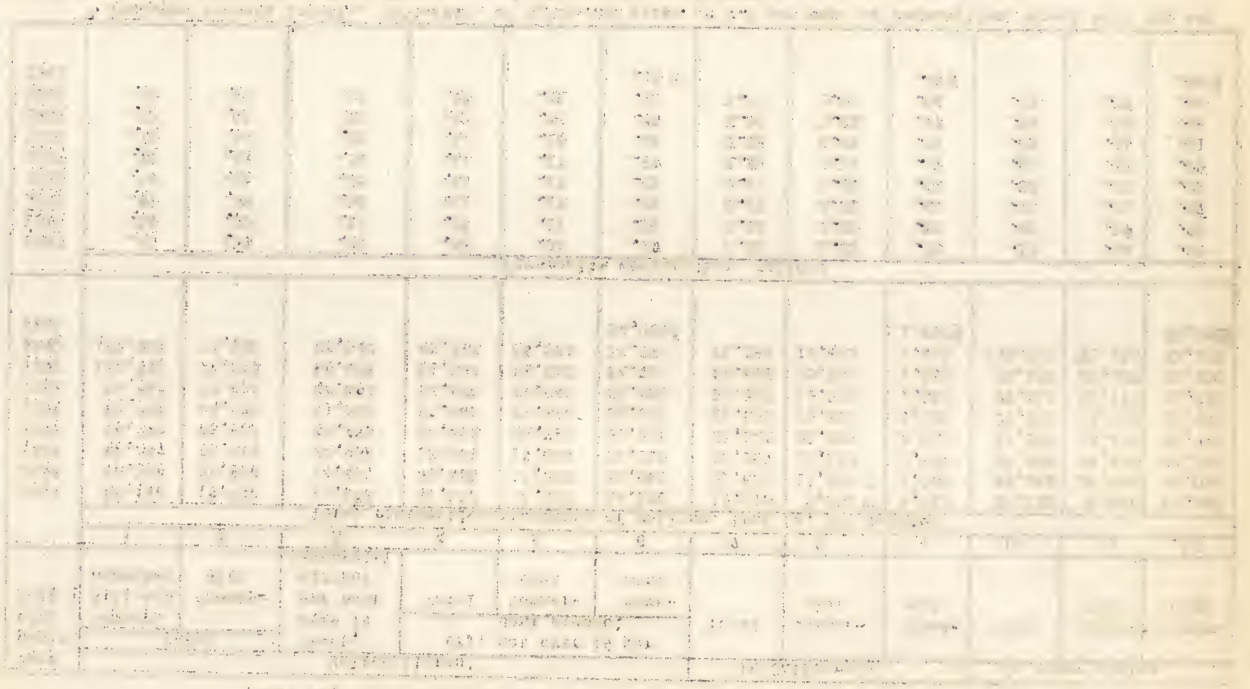


GRAFES

Table 17 United States and California Froduction, Stocks, Supply and Disappearance of Commercial Still Wine, Average 1999-1913, and Annual 1933-1941

\begin{tabular}{|c|c|c|c|c|c|c|c|c|c|c|c|c|}
\hline \multirow[b]{2}{*}{$\begin{array}{l}\text { Year } \\
\text { beginning } \\
\text { July I }\end{array}$} & \multicolumn{3}{|c|}{ Stocks, July 1} & \multicolumn{3}{|c|}{ Net finished production } & \multicolumn{3}{|c|}{ Total supply } & \multicolumn{3}{|c|}{ Disappearance } \\
\hline & Total & $\begin{array}{l}\text { Sweet - } \\
\text { over } 14 \% \\
\text { alcohol }\end{array}$ & $\begin{array}{l}\text { Dry -- } \\
\text { not ov- } \\
\text { er } 14 \%\end{array}$ & Total & $\begin{array}{l}\text { Sweet }- \\
\text { over } 14 \% \\
\text { alcohol }\end{array}$ & $\begin{array}{l}\text { Dry -- } \\
\text { not ov- } \\
\text { er } 14 \%\end{array}$ & Total & $\begin{array}{l}\text { Sweet }- \\
\text { over } 14 \% \\
\text { alcohol }\end{array}$ & $\begin{array}{l}\text { Dry -- } \\
\text { not ov- } \\
\text { er } 14 \%\end{array}$ & Total & $\begin{array}{l}\text { Sweet } \\
\text { over } 14 \% \\
\text { alcohol }\end{array}$ & $\begin{array}{l}\text { Dry - } \\
\text { not ov- } \\
\text { er } 14 \%\end{array}$ \\
\hline \multirow{4}{*}{$\begin{array}{l}\text { Average: } \\
\text { 1909-1913 } \\
\text { Annual: }\end{array}$} & 1 & 2 & 3 & 4 & 5 & 6 & 7 & 8 & 9 & 10 & 11 & 12 \\
\hline & \multirow{2}{*}{\multicolumn{12}{|c|}{ Thousands of gallons, that is, 000 omitted }} \\
\hline & \multicolumn{4}{|c|}{ United States } & & & & & & & \multirow{3}{*}{19,198} & \multirow{3}{*}{$30,247^{*}$} \\
\hline & -- & - & -- & 52,924 & 20,074 & $32,850^{*}$ & --1 & - & -- & & & \\
\hline $\begin{array}{l}\text { Hnnual: } \\
1933\end{array}$ & 25,542 & 11,597 & 13,945 & 39,239 & 17,583 & 21,656 & 64,781 & 29,180 & 35,601 & 14,613 & & \\
\hline 1934 & 50,168 & 19,064 & 30,504 & 41,580 & 27,311 & 14,669 & 92,148 & 46,975 & 45,173 & 35,671 & 23,396 & 12,275 \\
\hline 1935 & 56,477 & 23,579 & 32,898 & 69,821 & 55,363 & 14,458 & 126,298 & 78,942 & 47,356 & 47,826 & 31,855 & 15,971 \\
\hline 1936 & 78,472 & 47,087 & 31,385 & 52,031 & 36,490 & 15,541 & 130,503 & 83,577 & 46,926 & 62,395 & 41,208 & 21,187 \\
\hline 1937 & 68,108 & 42,369 & 25,739 & 95,308 & 59,416 & 35,892 & 163,416 & | 101,785 & 61,631 & 61,399 & 39,920 & 21,479 \\
\hline 1938 & 102,017 & 61,865 & 40,152 & 60,396 & 38,321 & 22,075 & 162,413 & 100,186 & 62,227 & 67,567 & 44,990 & 22,577 \\
\hline 1939 & 94,846 & 55,196 & 39,650 & 80,776 & 58,567 & 22,211 & $175,6 \approx 4$ & 113,763 & 61,861 & 82,379 & 57,583 & 24,796 \\
\hline 1940 & 93,245 & 56,180 & 37,065 & 113,998 & 82,865 & 31,133 & 207,243 & 139,045 & 68,198 & 89,356 & 62,331 & 27,025 \\
\hline $1941+$ & 117,887 & 76,714 & 41,173 & 118,000 & $81,000+$ & $37,000 t$ & $+235,887$ & $|+157,714|$ & $78,173+1$ & & & \\
\hline Average: & \multicolumn{11}{|c|}{ California } & \multirow{3}{*}{-} \\
\hline $1909-1913$ & -- & - & -- & 43,595 & 19,161 & $24,434^{*}$ & - & - & -- & --1 & -- & \\
\hline Annual: & & & & & & & & & & & & \\
\hline 1933 & $2 \approx, 620$ & 10,351 & 12,269 & 35,679 & 16,052 & 19,627 & 58,299 & 26,403 & 31,896 & 12,974 & 8,640 & 4,334 \\
\hline 1934 & 45,325 & 17,763 & 27,562 & 37,005 & 25,928 & 11,077 & $8 \%, 330$ & 43,691 & 38,639 & 34,944 & 23,366 & 11,578 \\
\hline 1935 & 47,386 & 20,325 & 27,061 & 65,690 & 54,013 & 11,677 & 113,076 & 74,338 & 38,738 & 45,206 & 31,696 & 13,510 \\
\hline 1936 & 67,870 & 42,642 & 25,228 & 46,679 & 34,700 & 11,979 & 114,549 & 77,342 & 37,207 & 58,212 & 40,574 & 17,638 \\
\hline 1937 & 56,337 & 36,768 & 19,569 & 85,351 & 57,302 & 28,049 & 141,688 & 94,070 & 47,618 & 54,363 & 38,718 & 15,645 \\
\hline 1938 & 87,325 & 55,352 & 31,973 & 50,342 & 35,581 & 14,761 & 137,667 & 90,933 & 46,734 & 57,155 & $42,6 \div 8$ & 14,527 \\
\hline 1939 & 80,512 & 48,305 & 32,207 & 71,478 & 55,304 & 16,174 & 151,990 & 103,609 & 48,381 & 74,759 & 54,862 & 19,897 \\
\hline 1940 & 77,231 & 48,747 & 28,484 & 100,818 & 79,008 & 21,810 & 178,049 & 127,755 & 50,294 & 78,511 & 60,355 & 18,156 \\
\hline $1941+$ & 99,538 & 67,400 & 32,138 & 104,00 ot & $78,000+$ & $26,000+$ & 203,53 है & $145,400^{\circ}$ & $58,138+1$ & & & \\
\hline
\end{tabular}

* Data on champugne and otherisparkling wines included for 1909-1913 but excluded for 1933-1941.

+ Rough prelininary 1941 production estir.tes subject to considerable revision.

Source of date: Compiled by S. W. Shear, Giannini Foundation. Averages for 1909-1913 from Giannini Foundation Mimeo. Report No. 34, June 1934. Data for 1933-1941: Cols. 1-3, from reports of the Bureau of Internal Revenue; cols. 4-6 besed on these reports; cols. 7-12, calculated from dets in cols. 1-6. 


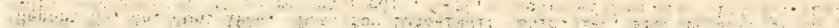

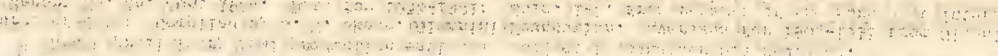

10.04 a

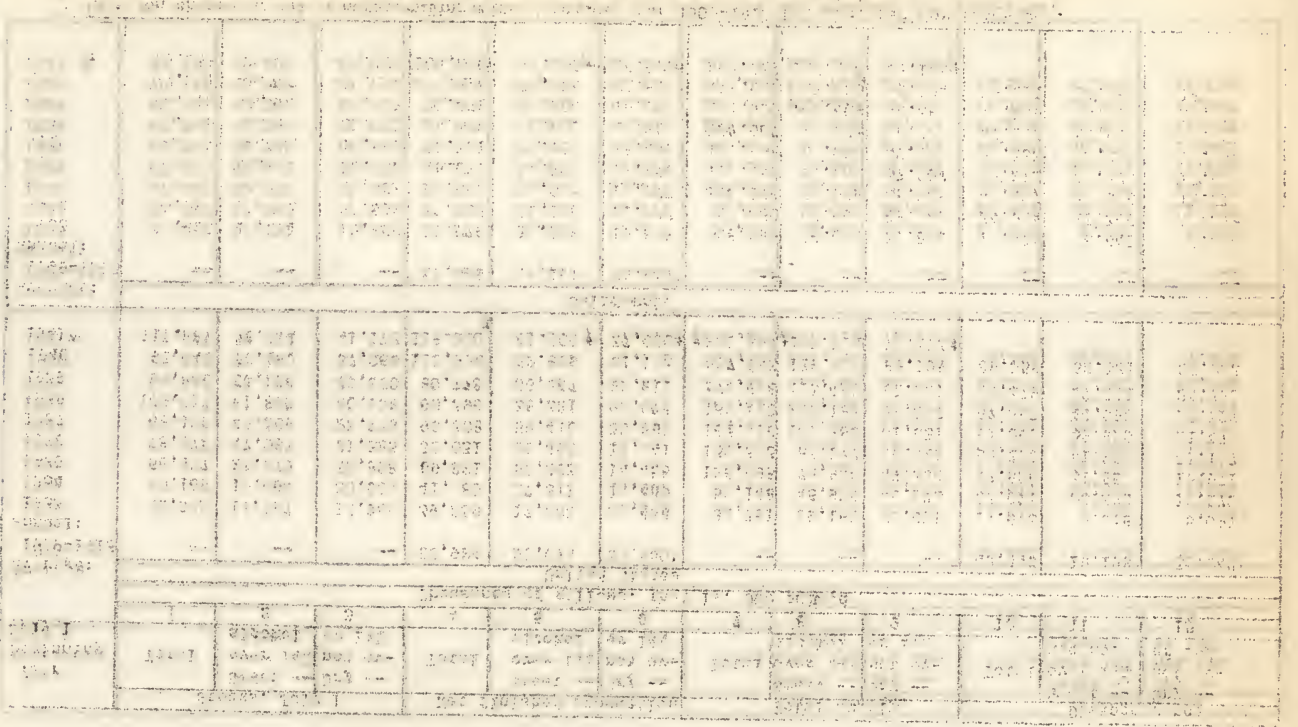

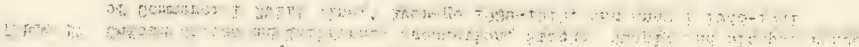

if 
GRAPES

Table 18, Supply and Disappearance of Californiz Fruit Brandy,* Averages 1890-1918, Annual 1933-1941

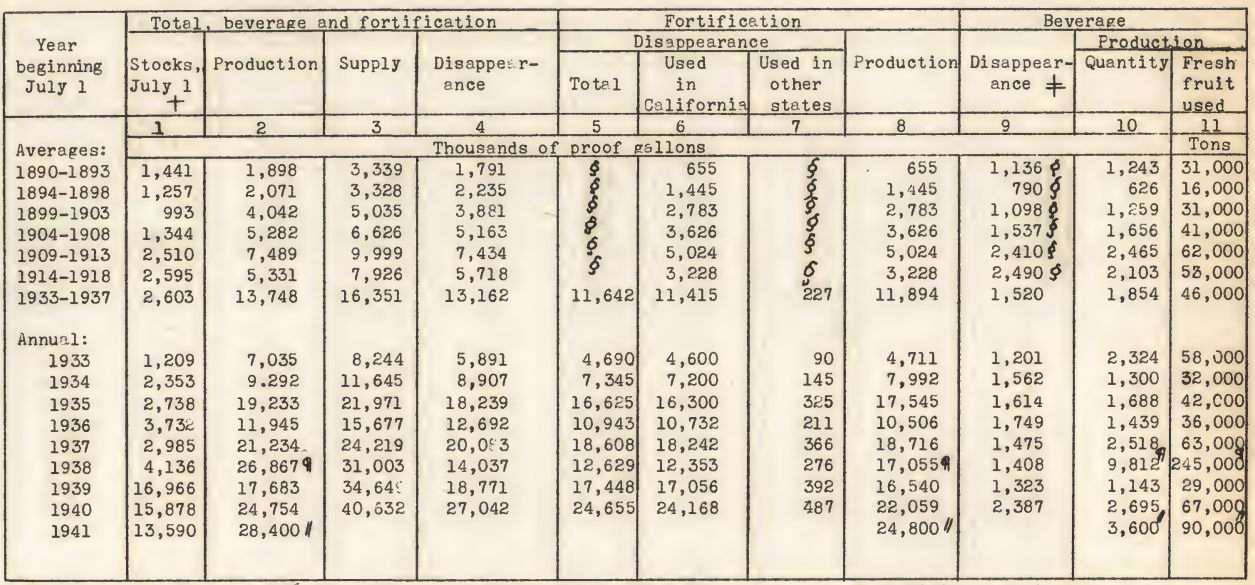

* $\mathrm{s}$ average of about $99 \%$ of California fruit brandy is grape brandy.

// Preliminary estimates

+ stocks remaining in C9lifornit speciel End bonded warehouses, 1890-1918; but only in bonded warehouses, $1933-1941$.

\# Includes annuil exports of from 1 to 319 thousand gallons, 1890-1918, and less then 500 g-110ns, $1933-1937$.

f The small quantities of California brandy used for fortificstion in stites other thü california ere not av ilable separately in 1890-1918, tut included in diseppearance of beverse brandy.

91938 includes lurge quantities of fortifying and beverage brandy made under the iine-Grupe Prorate irogram. Sources of data: Compiled by S.W. Shear, Giannini Foundation of Agricultural economics, 1890-1918 based on Shear, S. W. and G. G. Pearce, Supply and Frice Trends in theCalifornia Wine-Grape Industry, Table 35, June 1934; $1933-1941$ based on data of Bureau Internal Revenue, except cols. 8 and 10 from the Wine Institute. 


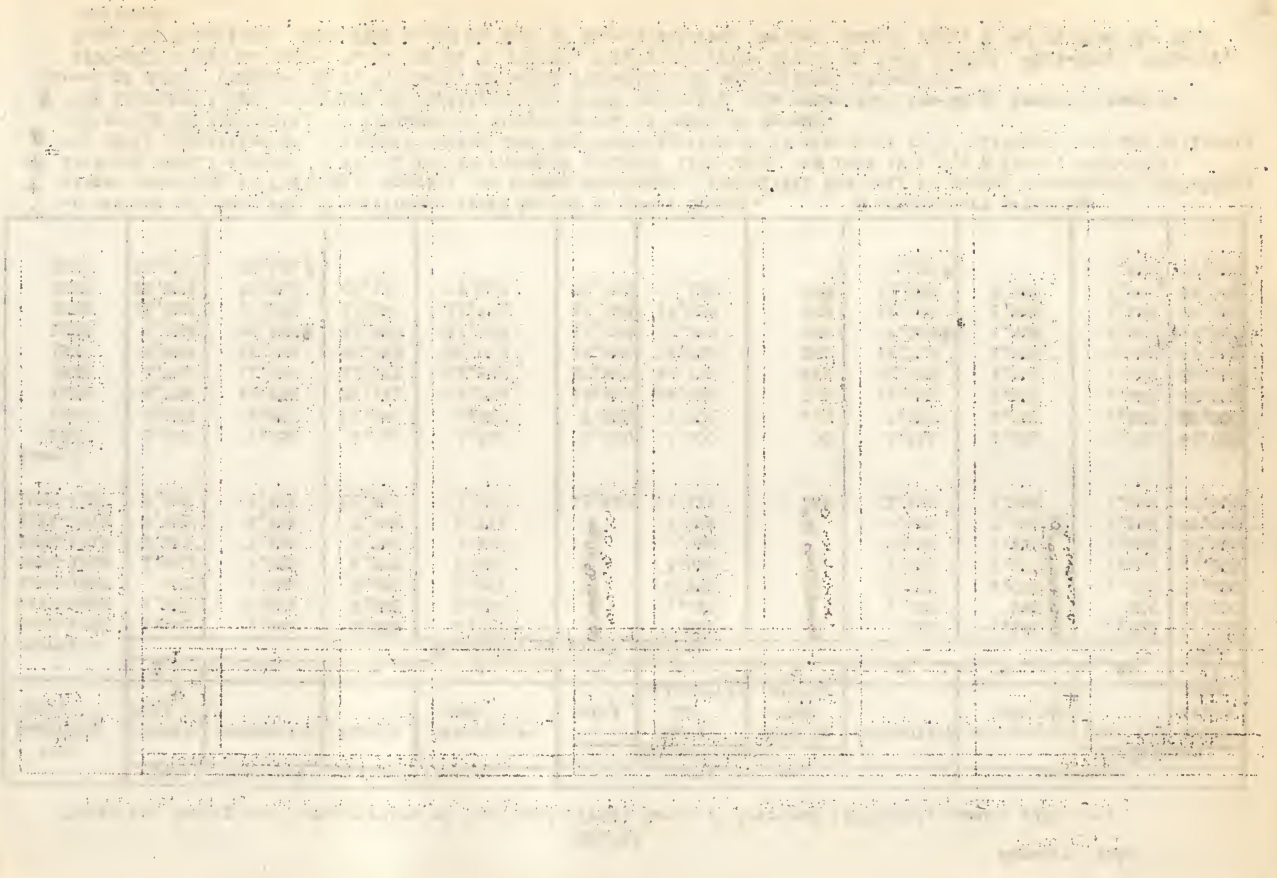


Table 19

United States Imports* of Still and Sparkling wines and Brandy by chief Countries of Origin, Avera,e 19-9-1913 and Annually 1934-1941

\begin{tabular}{|c|c|c|c|c|c|c|c|c|}
\hline \multirow{2}{*}{\begin{tabular}{|c|} 
Year \\
beginning \\
July 1 \\
\end{tabular}} & \multicolumn{8}{|c|}{ Still wine, includin vermouth } \\
\hline & Total & France & Italy & Spain & Portugal & cermany & Japan & Other \\
\hline \multirow{3}{*}{$\begin{array}{l}\text { Averages: } \\
1909-1913 \\
1934-1938\end{array}$} & \multicolumn{8}{|c|}{ Tine gallons } \\
\hline & $6,718,000$ & 772,000 & $3,000,000$ & $1,153,000$ & 175,000 & $|387,000|$ & 543,000 & 188,000 \\
\hline & $2,920,972$ & 863,708 & 995,157 & 478,105 & 110,425 & 150,233 & 138,375 & 176,968 \\
\hline \multicolumn{9}{|l|}{ Annual: } \\
\hline 1934 & $2,433,759$ & 711,541 & 708,327 & 340,588 & 92,428 & $1 \cup 0,252$ & 172,033 & 248,090 \\
\hline 1935 & $2,517,947$ & 707,428 & 780,248 & 427,946 & 81,553 & 135,626 & 145,478 & 239,668 \\
\hline 1936 & $3,458,588$ & 999,834 & $1,191,594$ & 604,059 & 119,513 & 164,872 & 138,276 & 240,840 \\
\hline 1937 & $3,046,152$ & 902,183 & $1,139,474$ & 516,416 & 125,368 & 155,534 & 124,132 & 83,015 \\
\hline 1938 & $3,148,014$ & 997,555 & $1,155,639$ & 501,518 & 133,262 & 174,800 & 111,955 & 73,217 \\
\hline 1939 & $3,898, \cup 84$ & $1,389,837$ & $1,541,481$ & 614,962 & 146,478 & 23,089 & 109,952 & 72,885 \\
\hline 1940 & $1,930,933$ & 425,912 & 388,300 & 554,892 & 139,000 & 25,715 & 76,524 & 325,530 \\
\hline \multicolumn{9}{|l|}{ July-Sept. } \\
\hline 1938 & 473,228 & 155,326 & 181,492 & 68,252 & 14,829 & 21,192 & 25,327 & 6,810 \\
\hline 1939 & 724,757 & 284,316 & 281,747 & 93,006 & 21,567 & 4,399 & 25,846 & 13,876 \\
\hline 1940 & 432,387 & 163,016 & 119,619 & 96,282 & 19.600 & 4,323 & 13,380 & 16,079 \\
\hline \multirow[t]{4}{*}{1941} & 390,303 & 40,717 & 30,121 & 121,751 & $30,0 \cup 3$ & 5,791 & 6,207 & 155,653 \\
\hline & \multicolumn{4}{|c|}{ Sparkling wines, includin ohampagne } & \multicolumn{4}{|c|}{ Brandy } \\
\hline & Total & France & Italy & other & Total & fracoe & Spain & Other \\
\hline & \multicolumn{4}{|c|}{ Wine gellons } & \multicolumn{4}{|c|}{ roor gallons } \\
\hline Averages : & & & & & & & & \\
\hline $\begin{array}{l}1909-1913 \\
1934-1938\end{array}$ & 692,000 & 618,000 & 5,000 & 69,000 & 569,000 & 466,000 & 5,000 & 98,000 \\
\hline $1934-1938$ & 435,539 & 399,095 & 10,609 & 25,835 & 610,099 & 553,204 & 20,018 & 36,877 \\
\hline \multicolumn{9}{|l|}{ Annual: } \\
\hline 1934 & 285,589 & 221,467 & 6,512 & 57,610 & 457,468 & 394,306 & 14,015 & 49,087 \\
\hline $\begin{array}{l}1935 \\
1936\end{array}$ & $\begin{array}{l}278,638 \\
564,783\end{array}$ & $\begin{array}{l}237,116 \\
521,564\end{array}$ & $\begin{array}{r}4,775 \\
15,960\end{array}$ & $\begin{array}{l}36,747 \\
27,259\end{array}$ & $\begin{array}{l}462,104 \\
730,984\end{array}$ & $\begin{array}{l}415,076 \\
652,046\end{array}$ & 15,671 & $\begin{array}{l}31,357 \\
50,407\end{array}$ \\
\hline 1937 & 538,749 & 522,104 & 13,344 & 3,301 & 700,391 & 037,023 & 28,724 & 34,644 \\
\hline 1938 & 509,936 & 493,225 & 12,454 & 4,257 & 699,547 & 666,906 & 13,750 & 18,891 \\
\hline 1939 & 641,154 & 624,701 & 10,884 & 5,569 & 882,182 & 055,572 & 19,085 & 7,525 \\
\hline 1940 & 254,216 & 232,814 & 6,784 & 14,618 & 502,354 & 375,941 & 85,823 & 40,590 \\
\hline \multicolumn{9}{|l|}{ July-Sept. } \\
\hline 1938 & 69,332 & 67,664 & 1,143 & 525 & 114,930 & 110,175 & 2,120 & 2,635 \\
\hline 1939 & 129,238 & 125,435 & 3,035 & 768 & 206,584 & 202,835 & 2,571 & 1,178 \\
\hline 1940 & 77,581 & 74,930 & 2,601 & 50 & 140,725 & 123,269 & 13,549 & 907 \\
\hline 1941 & 19,459 & 15,893 & 170 & 3,396 & 81,450 & 33,803 & 13,303 & 34,290 \\
\hline
\end{tabular}

* Dutiable irports only for consumption except 1909-1913 inolude reexports.

+ Data for Germany include Austria beginning :ay 6, 1938, and certain parts of Czechoslovakia and ienel acquired by Germany since then.

Sources of data: Compiled by S. . . Shear, Giannini Foundation of Asrioultural Economics. 1909-1913 from Foreign Commerce and Navigation of United States. 1934-1941 from U. S. Bureau Forei $n$ and Domestic Commerce, largely from Imports of Distilled

Liquors, Wines, Cordials, and alt liquors, mimeo. monthly 0. 3063. 
$\therefore+1+1+1+n$

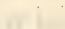

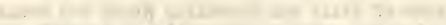

(1)

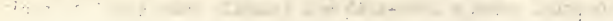

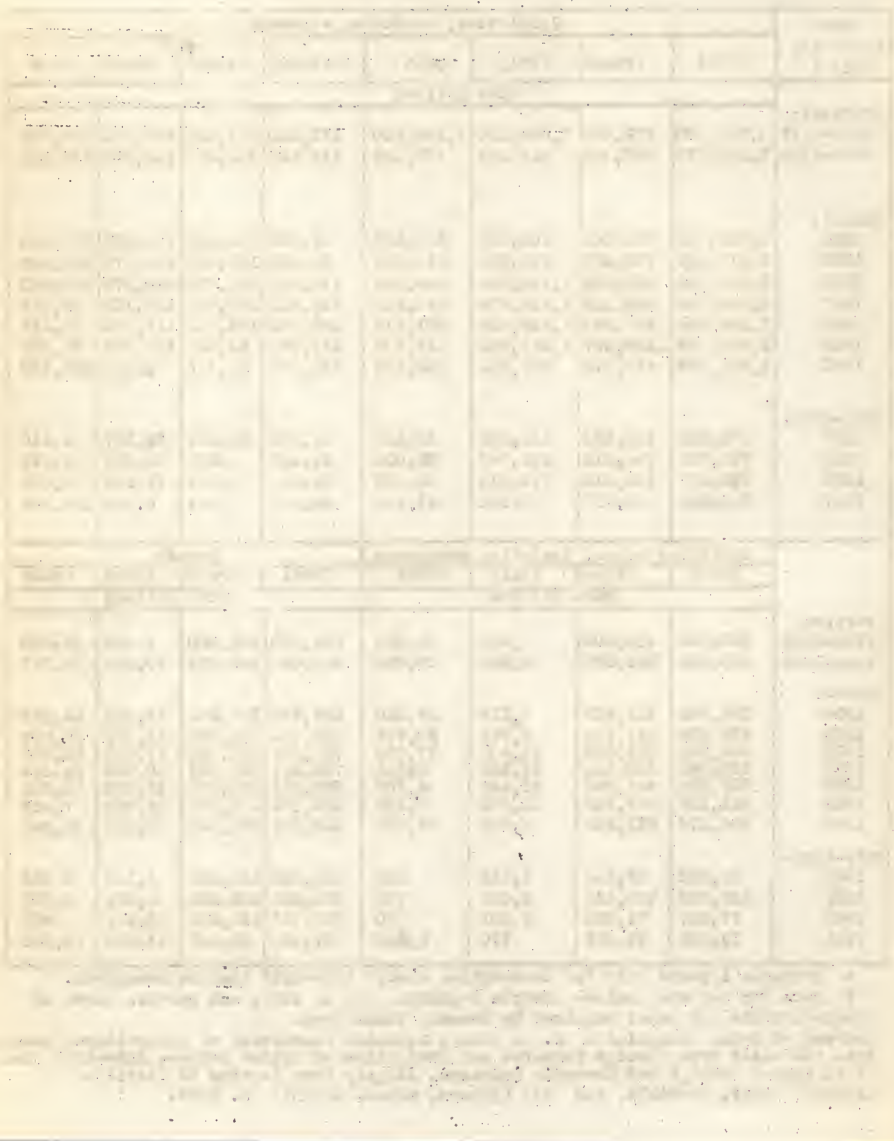


University of California, College of Agriculture

84. Agricultural Experiment Station, Berkeley, January, 1942

\section{DECIDUOUS FRUIT STATISTICS}

\section{PEACHES}

Table 1. California Peaches: Bearing Acreage, Production, Condition, Yield and Farm Value, 1928-1941

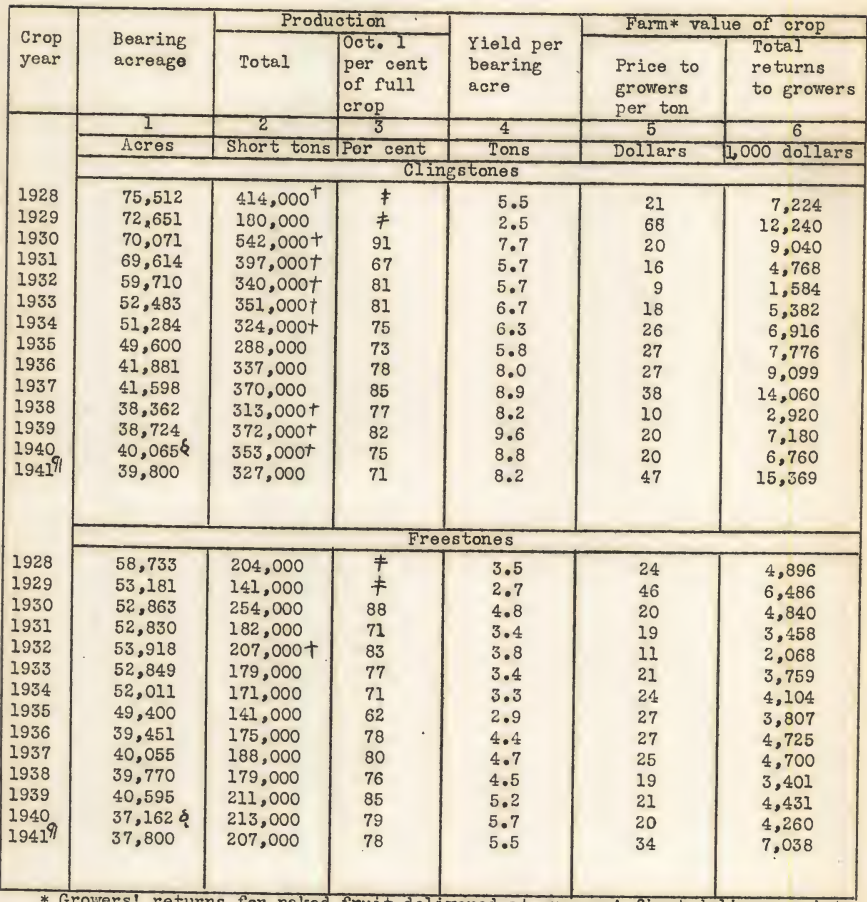

* Growers ${ }^{\prime}$ returns for naked fruit delivered at growers' first delivery point.

+ Includes unharvested tonnage: Clings - 1928, 70,000; 1930, 238,300; 1931, 193,$500 ; 1932,164,000$; $1933,85,700 ; 1934,58,000 ; 1938,21,000 ; 1939,13,000$; $1940,15,000$. Frees - 1930, 12,000; 1932, 19,000. See table 2, footnote $t$, for clingstones unharvested but purchased in 1930, 1931 and 1933.

FCondition for all California peuches in 1928, 87 per cent; 1929, 44 per cent.

\&Nonbearing acreuge 1940: Clings, 11,209; Frees, 7,710. 9l Preliminary. Source of data: Compiled by S. W. Shear, Giannini Foundation of Agricultural

Economics, from California Crop Reporting Service, except cols. 4 and 6 calculated. 


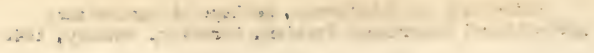

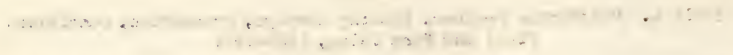

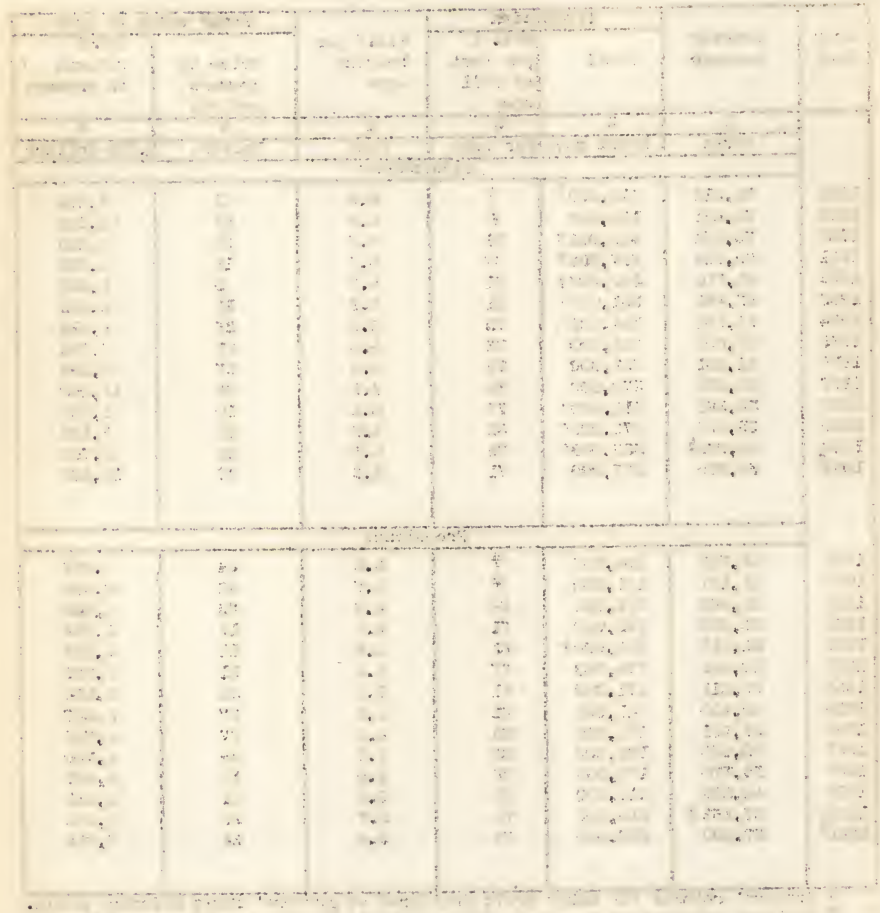

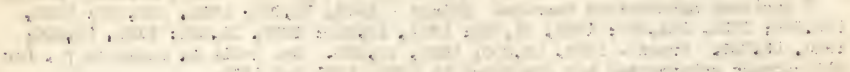

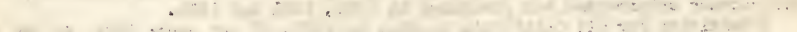

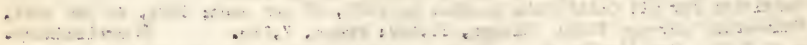

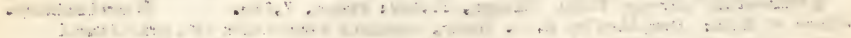

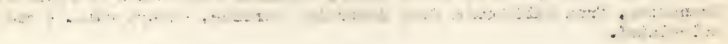




\section{PEFCHES}

Trable 2

California Clingstone Peaches: Production and Utilization, 1909-1941

\begin{tabular}{|c|c|c|c|c|c|c|c|}
\hline \multirow{2}{*}{$\begin{array}{l}\text { Crop } \\
\text { year }\end{array}$} & \multicolumn{3}{|c|}{ Production } & \multirow[b]{2}{*}{ Canned } & \multirow[b]{2}{*}{ Dried } & \multicolumn{2}{|c|}{ Used fresh } \\
\hline & Total & Unha rves ted & Harves ted & & & $\begin{array}{l}\text { Out of } \\
\text { state }\end{array}$ & In state* \\
\hline & \multicolumn{7}{|c|}{ Short tons, fresh equivalent weight } \\
\hline $\begin{array}{l}\text { terages: } \\
\text { 1909-1913 }\end{array}$ & 38,800 & 0 & & & 0 & & 3.180 \\
\hline $1914-1918$ & 74,800 & 0 & $\begin{array}{l}38,800 \\
74,800\end{array}$ & $\begin{array}{l}35,340 \\
68,160\end{array}$ & $\begin{array}{l}0 \\
0\end{array}$ & $\begin{array}{l}280 \\
820\end{array}$ & $\begin{array}{l}3,180 \\
5,820\end{array}$ \\
\hline $1919-1924$ & 145,000 & 0 & 145,000 & 132,820 & 0 & 2,580 & 9,600 \\
\hline $1925-1929$ & 295,800 & 27,000 & 268,800 & 252,700 & 0 & 4,580 & 11,520 \\
\hline $1930-1934$ & 390,800 & $147,900 t$ & 242,900 & 206,840 & 14,240 & 9,020 & 12,800 \\
\hline $1935-1939$ & 336,000 & 6,800 & 329,200 & 274,420 & 34,880 & 3,040 & 16,860 \\
\hline \multicolumn{8}{|l|}{ Annual: } \\
\hline 1919 & 128,000 & 0 & 128,000 & 118,500 & 0 & 1,700 & 7,800 \\
\hline 1920 & 132,000 & 0 & 132,000 & 121,100 & 0 & 2,500 & 8,400 \\
\hline 1921 & 109,000 & 0 & 109,000 & 96,800 & 0 & 2,800 & 9,400 \\
\hline 1922 & 195,000 & 0 & 195,000 & 182,400 & 0 & 2,600 & 10,000 \\
\hline 1923 & 169,000 & 0 & 169,000 & 153,300 & 0 & 4,800 & 10,900 \\
\hline 1924 & 137,000 & 0 & 137,000 & 124,800 & 0 & 1,100 & 11,100 \\
\hline 1925 & 232,000 & 0 & 232,000 & 215,300 & 0 & 4,800 & 11,900 \\
\hline 1926 & 331,000 & 0 & 331,000 & 317,500 & 0 & 700 & 12,800 \\
\hline 1927 & 322,000 & 65,000 & 257,000 & 235,400 & 0 & 9,100 & 12,500 \\
\hline 1928 & 414,000 & 70,000 & 344,000 & 322,000 & 0 & 7,800 & 14,200 \\
\hline 1929 & 180,000 & 0 & 180,000 & 173,300 & 0 & 500 & 6,200 \\
\hline 1930 & 542,000 & $238,300+$ & 303,700 & 288,100 & 0 & 8,700 & 6,900 \\
\hline 1931 & 397,000 & $193,500 t$ & 203,500 & 181,500 & 8,100 & 7,000 & 6,900 \\
\hline 1932 & 340,000 & 164,000 & 176,000 & 139,400 & 3,600 & 12,900 & 20,100 \\
\hline 1933 & 351,000 & $85,700 t$ & 265,300 & 233,900 & 11,500 & 7,300 & 12,600 \\
\hline 1934 & 324,000 & 58,000 & 266,000 & 191,300 & 48,000 & 9,200 & 17,500 \\
\hline 1935 & 288,000 & 0 & 288,000 & 243,400 & 34,400 & 1,200 & 9,000 \\
\hline 1936 & 337,000 & 0 & 337,000 & 265,000 & 50,000 & 2,600 & 19,400 \\
\hline 1937 & 370,000 & 0 & 370,000 & 322,700 & 34,000 & 2,900 & 10,400 \\
\hline 1938 & 313,000 & 21,000 & 292,000 & 241,800 & 26,000 & 2,900 & 21,300 \\
\hline 1939 & 372,000 & 13,000 & 359,000 & 299,200 & 30,000 & 5,600 & 24,200 \\
\hline 1940 & 353,000 & 15,000 & 338,000 & 283,200 & 41,400 & 3,000 & 10,400 \\
\hline $1941 \neq$ & 327,000 & 0 & 327,000 & 310,000 & 9,000 & 1,000 & 7,000 \\
\hline & & & & & & & \\
\hline
\end{tabular}

* Includes small quantities used fresh on the farm.

t Includes quantities purchased but not harvested under the industry control programs as follows: 1930, 148,300 tons; 1931, 94,500 tons; and 1933, 33,700 tons. F Preliminary 1941 estimates; utilization data are unofficial es timates.

Source of data: Compiled by S. W. Shear, Giannini Foundation of Agricultural

Economics, University of California, from releases of the California Cooperutive Crop Reporting Service. 



\section{PEACHES}

Table 3

California Freestone Peaches: Production and Utilization, 1909-1941

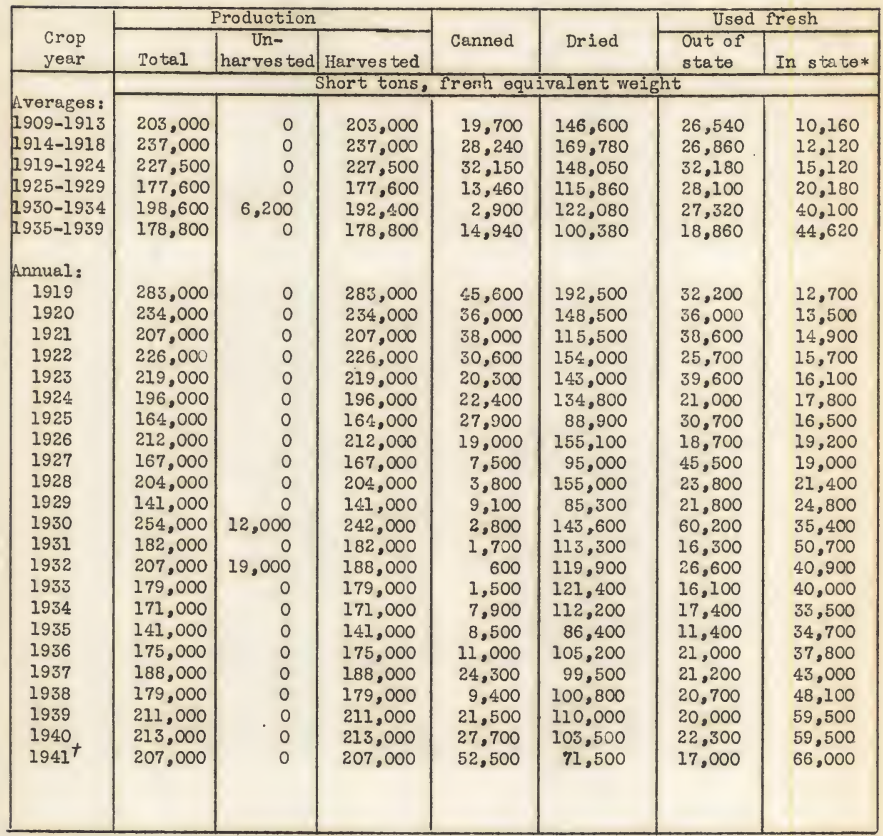

* Includes small quantities used fresh on the farm.

TPreliminary 1941 estimates; utilization data are unofficial estimates.

Source of data: Compiled by S. W. Shear, Giannini Foundation of Acricultural Economics, University of California, from releases of the California Cooperative Crop Reporting Service. 


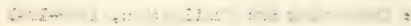

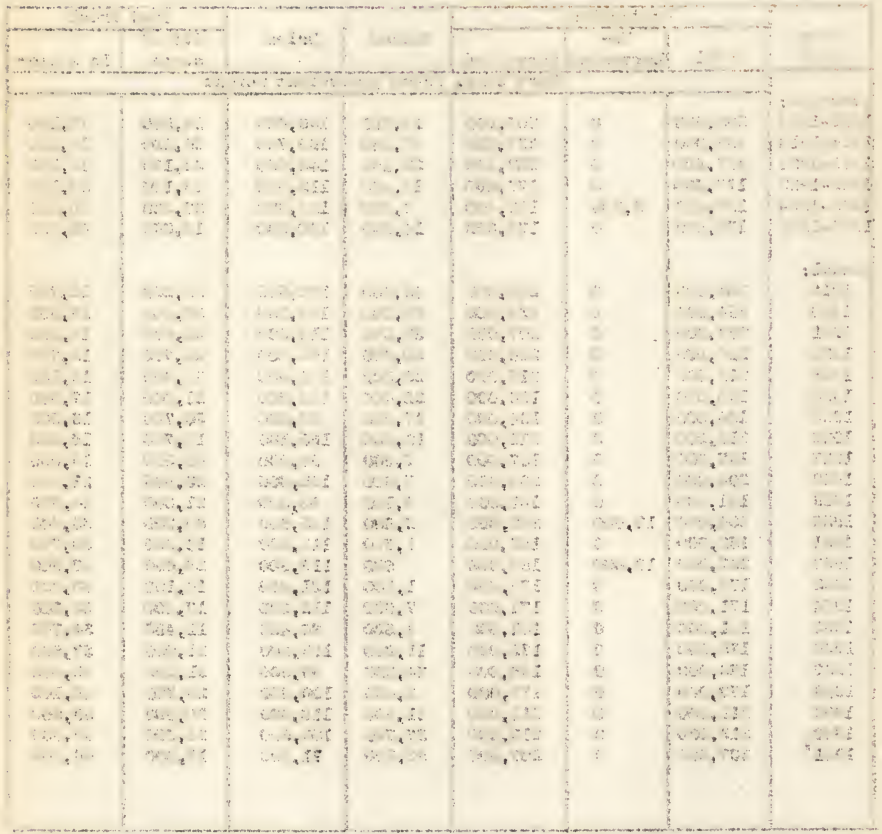

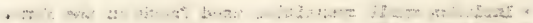

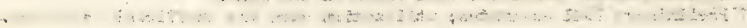

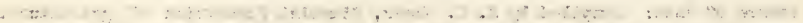

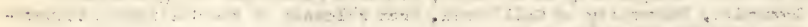

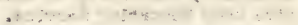




\section{PEACHES}

Table 4

United States Total* Production of Peaches, 1921-1941

\begin{tabular}{|c|c|c|c|c|c|}
\hline \multirow[b]{2}{*}{ Crop year } & \multirow[b]{2}{*}{$\begin{array}{c}\text { Unitod States, } \\
\text { total }\end{array}$} & \multirow[b]{2}{*}{$\begin{array}{l}\text { Other } \\
\text { states* }\end{array}$} & \multicolumn{3}{|c|}{ California } \\
\hline & & & Total & Freestones & Clingstones \\
\hline & \multicolumn{5}{|c|}{ Short tons } \\
\hline $\begin{array}{l}\text { Averages: } \\
1924-1928\end{array}$ & $1,315,300$ & & 475,800 & 188,600 & 287,200 \\
\hline $1929-1933$ & $1,275,300$ & 720,700 & 554,600 & 192,600 & 362,000 \\
\hline $1934-1938$ & $1,255,300$ & 758,100 & 497,200 & 170,800 & 326,400 \\
\hline \multicolumn{6}{|l|}{ Annual: } \\
\hline 1921 & 793,500 & 477,500 & 316,000 & 207,000 & 109,000 \\
\hline 1922 & $1,385,700$ & 964,700 & 421,000 & 226,000 & 195,000 \\
\hline 1923 & $1,082,900$ & 694,900 & 388,000 & 219,000 & 169,000 \\
\hline 1924 & $1,248,100$ & 915,100 & 333,000 & 196,000 & 137,000 \\
\hline 1925 & $1,098,400$ & 702,400 & 396,000 & 164,000 & 232,000 \\
\hline 1926 & $1,600,400$ & $1,057,400$ & 543,000 & 212,000 & 331,000 \\
\hline 1927 & $1,044,400$ & 555,400 & 489,000 & 167,000 & 322,000 \\
\hline 1928 & $1,585,400$ & 967,400 & 618,000 & 204,000 & 414,000 \\
\hline 1929 & $1,073,700$ & 752,700 & 321,000 & 141,000 & 180,000 \\
\hline 1930 & $1,328,200$ & 532,200 & 796,000 & 254,000 & 542,000 \\
\hline 1931 & $1,849,200$ & $1,270,200$ & 579,000 & 182,000 & 397,000 \\
\hline 1932 & $1,039,000$ & 492,000 & 547,000 & 207,000 & 340,000 \\
\hline 1933 & $1,086,500$ & 556,500 & 530,000 & 179,000 & 351,000 \\
\hline 1934 & $1,144,400$ & 649,400 & 495,000 & 171,000 & 324,000 \\
\hline 1935 & $1,312,500$ & 883,500 & 429,000 & 141,000 & 288,000 \\
\hline 1936 & $1,139,600$ & 627,600 & 512,000 & 175,000 & 337,000 \\
\hline 1937 & $1,433,300$ & 875,300 & 558,000 & 188,000 & 370,000 \\
\hline 1938 & $1,246,700$ & 754,700 & 492,000 & 179,000 & 313,000 \\
\hline 1939 & $1,465,700$ & 882,700 & 583,000 & 211,000 & 372,000 \\
\hline 1940 & $1,306,300$ & 740,300 & 566,000 & 213,000 & 353,000 \\
\hline $1941 \neq$ & $1,670,600$ & $1,136,600$ & 534,000 & 207,000 & 327,000 \\
\hline & & & & & \\
\hline
\end{tabular}

* Includes California unharvested production as shown in tubles 2 and 3 and unharvested tonnage in other states: 1926, 33,600; 1928, 25,000; 1931, 10,000; $1932,2,000$; 1938, 6,000; 1939, 12,000; and 1941, 31,700. Noarly all peaches produced in states other than Cal ifornia are freestones and are shipped fresh as practioally no peaches are dried commercially in other states and an avernge of only about 8,900 tons were canned in "other states," 1936-1940.

† Includes 1,344 tons harvested in Virginia but not used, and 1,440 tons in Colorado diverted from narketing channels in 1940.

F Preliminary estimates.

Sources of data: Compiled by S. W. Shear, Giannini Foundation of fgricultural Economics, University of California, from latest official reports of the United States and California Crop Reporting Service. Data other than California converted from bushels at 48 pounds per bushel and rounded to nearest hundred tons. 


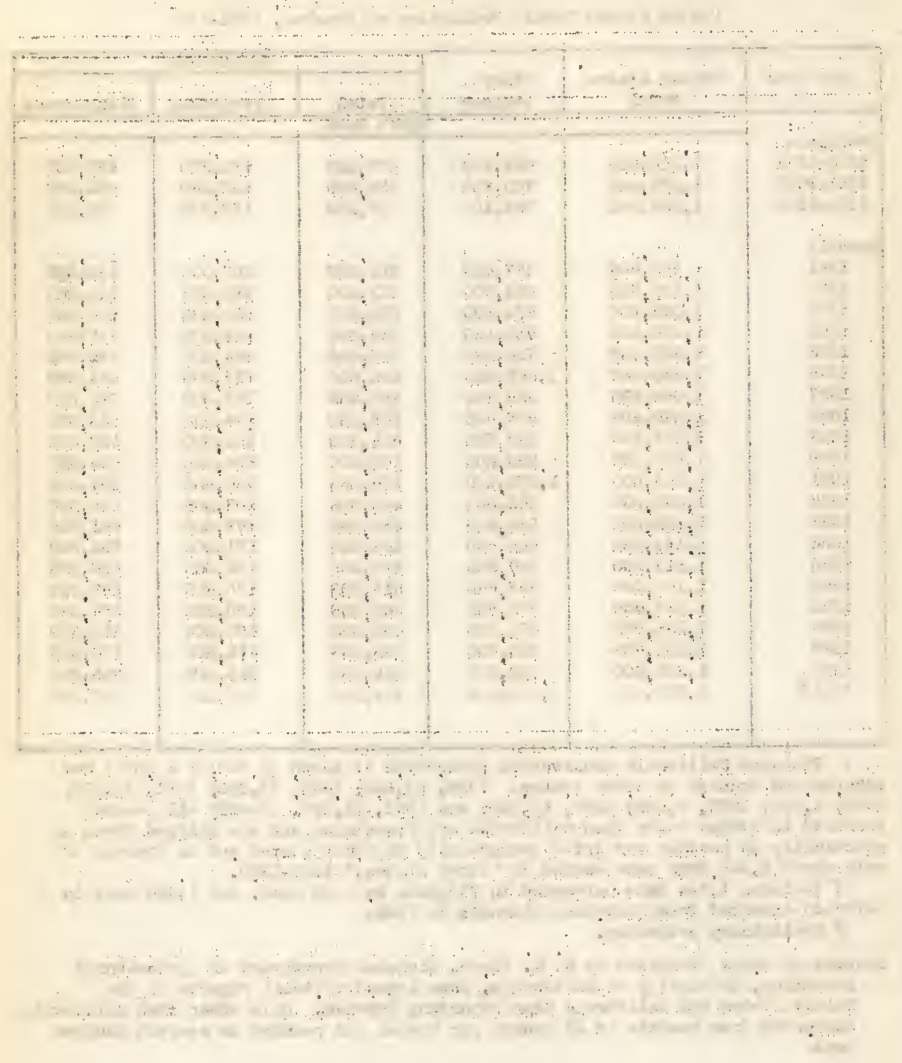


Table 5

Dried Peaches: California Production, Exports and Paciers' F.0.B. Prices, 1921-1941

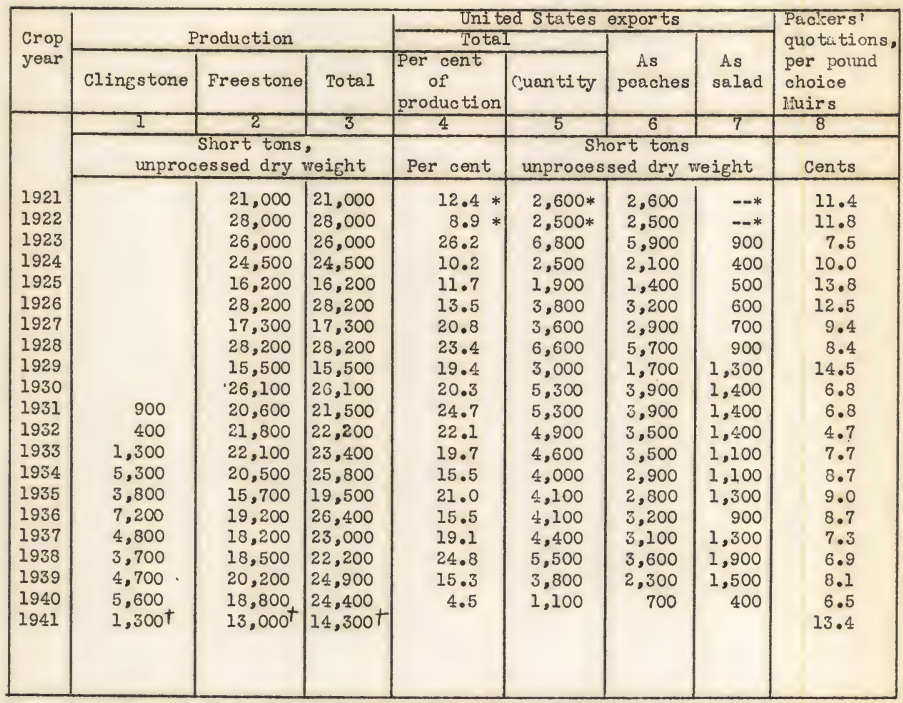

* Peach exports in salad are excluded in 1921 and 1922 as data not available. + Production for 1941 is a preliminary trade estimate subject to revision. Sources of data: Compiled by S. W. Shear, Giannini Foundation of Agricultural Economics, University of California.

Cols. 1-3: 1921-1933 from reports of the California Cooperative Crop Reporting Service. 1934-1940 are reliable trade estimates. Almost no clingstones were dried before 1931 and data a re not available.

Cols. 5-7, Exports of dried peaches and 21 per cent of exports of dried fruit salad, years beginning August 1 . Based on official data of United States Bureau of Foreign and Domestic Comerce, except salad in 1923-1929 estinated by S. $\pi$. Shear largely on inspections by Dried Fruit issociation of California.

Col. 8: Average of weekly quotations in the California Fruit Ilerrs, for August through December. 


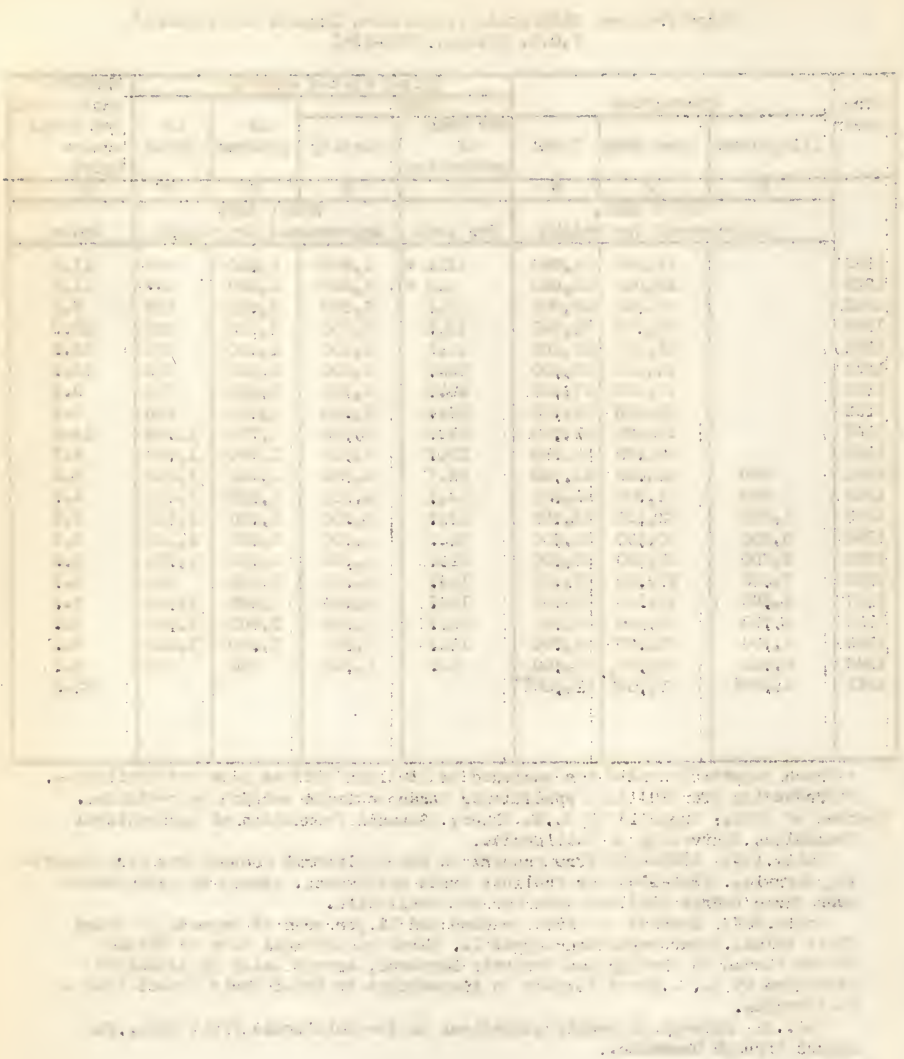


Table 6

Dried Peaches:* United States Domestic Exports by Countries of Destinatjon, Yeers Beginning July 1, 1930-1940

\begin{tabular}{|c|c|c|c|c|c|c|c|c|c|c|c|}
\hline $\begin{array}{l}\text { Country of } \\
\text { destination }\end{array}$ & $1930-31$ & $1931-32$ & $1932-33$ & $1933-34$ & $1934-35$ & $1935-36$ & $1936-37$ & $1937-38$ & $1938-39$ & $1939-40^{+}$ & $1940-41^{\#}$ \\
\hline & \multicolumn{11}{|c|}{ St.ort tons, net declared $\mathrm{dr}$; export weight } \\
\hline Total exports & 4,241 & 4,245 & 3,824 & 3,784 & 3,176 & 3,048 & 3,522 & 3,174 & 4,119 & 2,580 & $667 \neq$ \\
\hline Europe, total & 3,565 & 3,505 & 3,115 & 2,898 & 2,220 & 2,138 & 2,453 & 1,971 & 3,238 & 1,630 & 74 \\
\hline United Kingdom & 456 & 345 & 332 & 180 & 220 & 481 & 227 & 476 & 541 & 565 & 0 \\
\hline Other & 3,109 & 3,160 & 2,783 & 2,718 & 2,000 & 1,657 & 2,226 & 1,495 & 2,697 & 1,065 & 74 \\
\hline France & 810 & 941 & 894 & 627 & 814 & 1,080 & 1,454 & 792 & 1,109 & 160 & 0 \\
\hline Netherlands & 169 & 54 & 112 & 115 & 52 & 84 & 68 & 82 & 308 & 204 & 0 \\
\hline Belgium & 124 & 41 & 30 & 58 & 52 & 33 & 67 & 74 & 53 & 16 & 0 \\
\hline Norway & 5 & 40 & 38 & 20 & 24 & 18 & 36 & 30 & 61 & 41 & 0 \\
\hline Sweden & 42 & 189 & 211 & 132 & 102 & 186 & 404 & 263 & 311 & 291 & 73 \\
\hline Denmark & 224 & 595 & 148 & 212 & 92 & 34 & 20 & 22 & 582 & 249 & 0 \\
\hline Germany $\delta$ & 1,206 & 899 & 1,040 & 1,310 & 644 & 54 & 68 & 91 & 79 & 1 & 0 \\
\hline Italy & 428 & 345 & 188 & 151 & 113 & 10 & 2 & 4 & 34 & 7 & 0 \\
\hline Other & 101 & 56 & 122 & 93 & 107 & 158 & 107 & 137 & 160 & 96 & 1 \\
\hline $\begin{array}{l}\text { Except wurope, } \\
\text { total }\end{array}$ & 676 & 740 & 709 & 886 & $\$ 56$ & 910 & 1,069 & 1,203 & 881 & 950 & 593 \\
\hline Canada & 554 & 640 & 635 & 802 & 832 & 815 & 877 & 809 & 599 & 736 & 397 \\
\hline Other & 122 & 100 & 74 & 84 & 124 & 95 & 192 & 394 & 282 & 214 & 196 \\
\hline
\end{tabular}

* Do not include exports of dried peaches in dried fruit compote or salad. $\quad$ Preliminary.

F Nirs nontrs only, July 1940-March 1941 as data by countries of destination not availuble for later months. Total exporis for. July - June were 802 tons.

$\$$ Date for Germany include Austria beginning May 6, 1938, and certain parts of Czechoslovakia and Memel acquired by Germany since then.

Sources of deis: Compiled by S. H. Shear, Giannini Foundation of Agricultural Economics, University of California. 1930-1933: Data from U. S. Dept. Agr., Agricultural Statistics, 1937, p. 349.

1934-1939: From U. S. Dept. Agr., Office of Foreign Agricultural Relations.

1940: From U.S. Bureau of Foreign and Domestic Commerce, monthly mimeographed statements No. 3013. 


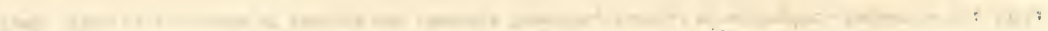

$\begin{aligned} & + \\ & \vdots\end{aligned} \ldots+\ldots$

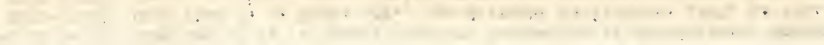

(20)

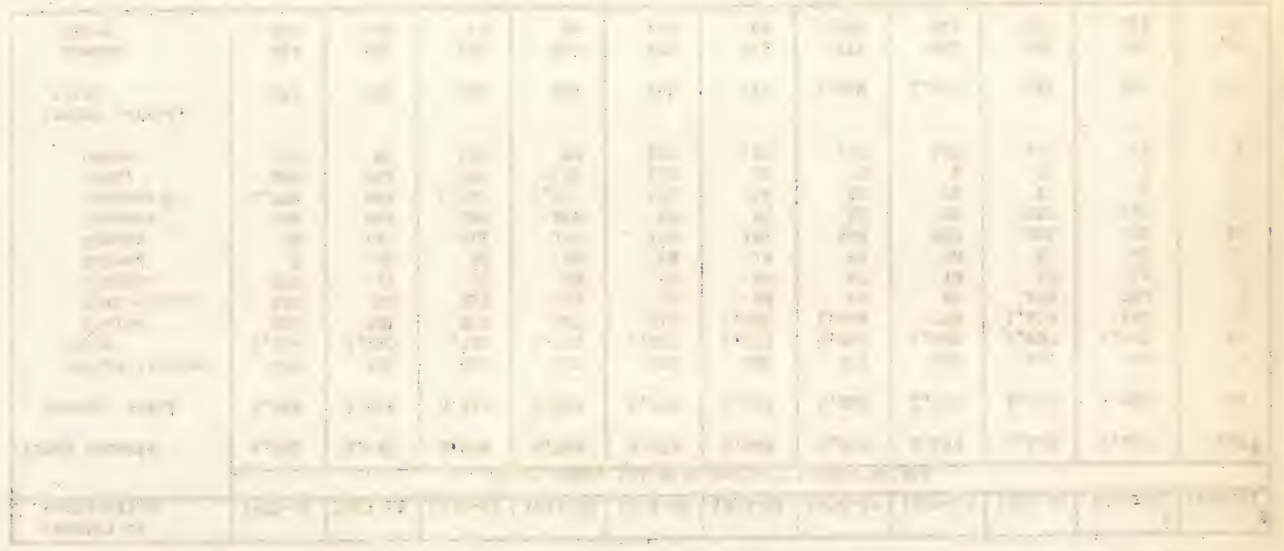

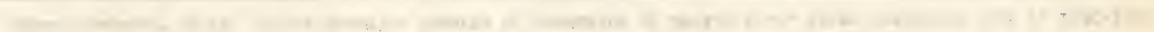


PELCHES

Table 7

Fresh Peaches: United States and California Carlot Shipnents and Delivered Eastem Price of California Elbertas, 1924-1941

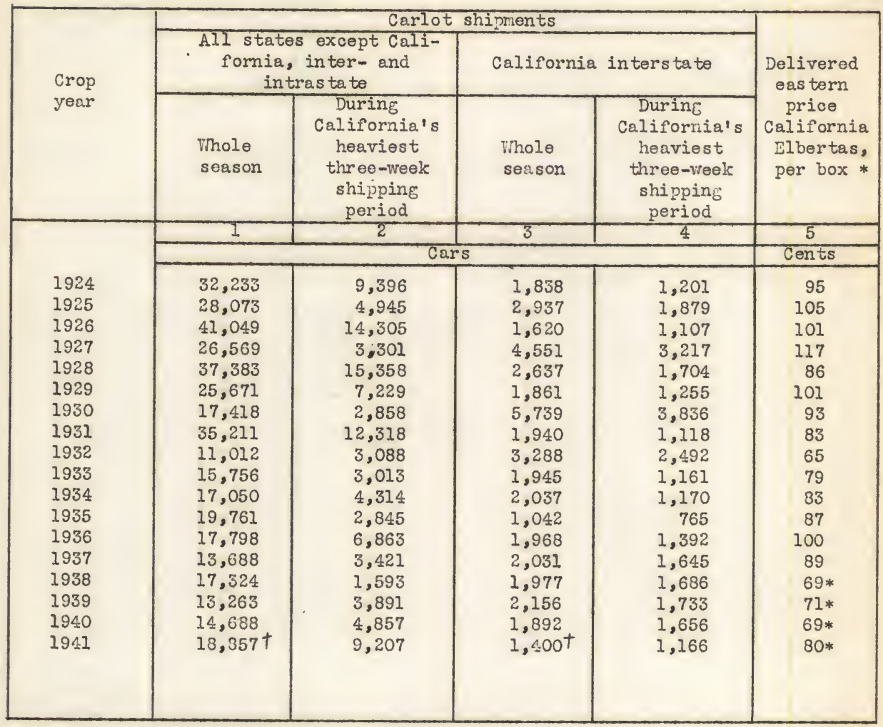

* Prices for 1924-1937 are for the old large box and for 1938-1941 for the short box now generally used which is about 10 per cont smaller than the old box. + Preliminary.

Sources of data: Compiled by S. W. Shear, Giamini Foundation of Agricultural Economics, University of California.

Cols. 1-4: Largely from mimeographed reports of the United States AEricultural Marketing Service and of the Federal-State Narket News Service.

Col. 5: Representative prices based upon private and auction sales of a large volume of Elbertas marlzeted by private and cooperative marleting organizations. 


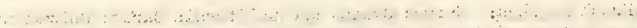

+...

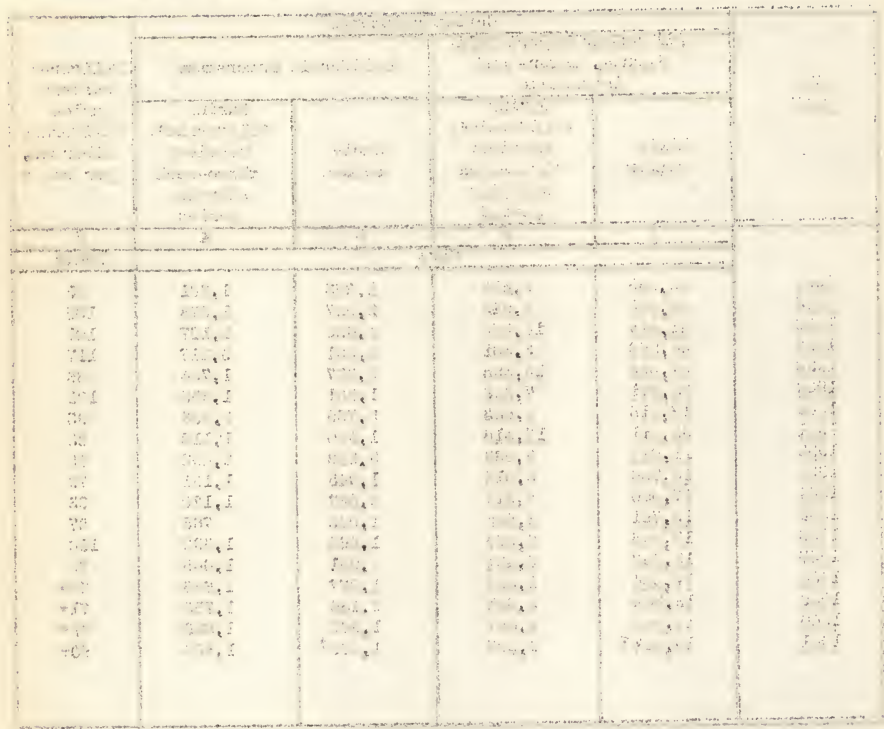

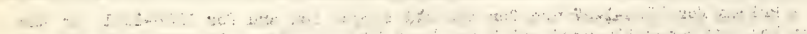

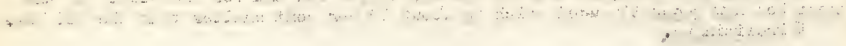

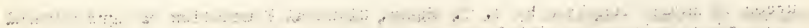

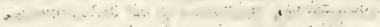

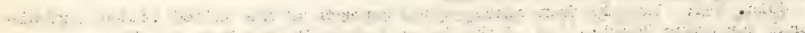

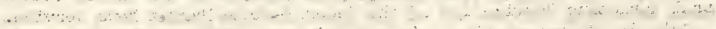

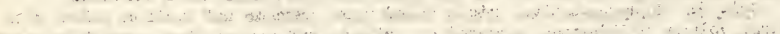

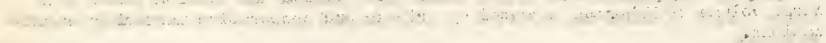


Table 8. California Cannec Peaches:* Pack, Carryover, Shipments, Exports, and Priccs, 1921-1941

\begin{tabular}{|c|c|c|c|c|c|c|c|c|c|}
\hline $\begin{array}{l}\text { Year } \\
\text { begin- } \\
\text { ning } \\
\text { June 1 } \\
\end{array}$ & $\begin{array}{c}\text { Fack } \\
*\end{array}$ & $\begin{array}{c}\text { Carryover } \\
\text { from } \\
\text { previous } \\
\text { year }\end{array}$ & $\begin{array}{c}\text { Available } \\
\text { for } \\
\text { shipment }\end{array}$ & $\begin{array}{l}\text { Carryover } \\
\text { into } \\
\text { following } \\
\text { year }\end{array}$ & $\begin{array}{l}\text { Shipments, } \\
\text { total }\end{array}$ & $\begin{array}{l}\text { United } \\
\text { States } \\
\text { exportst }\end{array}$ & $\begin{array}{l}\text { Domestic } \\
\text { shipments }\end{array}$ & $\begin{array}{l}\text { Frices } \\
\text { canners } \\
\text { received } \\
\text { per case } \\
\end{array}$ & $\begin{array}{l}\text { Usual grower } \\
\text { prices per ton, } \\
\text { No. } 1 \text { canning } \\
\text { clings }\end{array}$ \\
\hline & 1 & 2 & 3 & 4 & 5 & 6 & 7 & 8 & 9 \\
\hline & \multicolumn{7}{|c|}{ Thousands of cases, equivalent 24 . No. $2 \frac{1}{2}$ cans } & \multicolumn{2}{|c|}{ Dollars } \\
\hline 1921 & 5,633 & 920 & 6,553 & 326 & 6,227 & 1,108 & 5,119 & 4.13 & 35.00 \\
\hline 1922 & 8,784 & 326 & 9,110 & 2,109 & 7,001 & 1,214 & 5,787 & 4.25 & 60.00 \\
\hline 1923 & 7,158 & 2,109 & 9,267 & 1,575 & 7,692 & 1,147 & 6.545 & 3.67 & 30.00 \\
\hline 1924 & 6,141 & 1,575 & 7,716 & 798 & 6,918 & 1,281 & 5,637 & $4 \cdot 21$ & 45.00 \\
\hline 1925 & 10,143 & 798 & 10,941 & 574 & 10,367 & 1,856 & 8.511 & 3.78 & 35.00 \\
\hline 1926 & 14,059 & 574 & 14,633 & 3,906 & 10,727 & 1,681 & 9,046 & 3.66 & 40.00 \\
\hline 1927 & 10,813 & 3,906 & 14,719 & 1,516 & 13,203 & 2,040 & 11,163 & 3.17 & 22.50 \\
\hline 1928 & 14,596 & 1,516 & 16,112 & 3,149 & 12,963 & 2,163 & 10,800 & 3.21 & 20.00 \\
\hline 1929 & 8,100 & 3,149 & 11,249 & 1,677 & 9,572 & 1,727 & 7,845 & 4.08 & 80.00 \\
\hline 1930 & 13,294 & 1,677 & 14,971 & 3,951 & 11.020 & 1,618 & 9,402 & 2.88 & 20.00 \\
\hline 1931 & 8,421 & 3,951 & 12.372 & 4.845 & 7,527 & 1,469 & 6,058 & 2.55 & 14.50 \\
\hline 1932 & 6,438 & 4,845 & 11.283 & 1,361 & 9,922 & 1,733 & 8,189 & 1.97 & 6.50 \\
\hline 1933 & 10,309 & 1,361 & 11,670 & 2,390 & 9,280 & 1,799 & 7,481 & 2.31 & 20.00 \\
\hline 1934 & 8,598 & 2,390 & 10,988 & 1,856 & 9,132 & 1,126 & 8,006 & 2.69 & 30.00 \\
\hline 1935 & 23.216 & 1,856 & 13,072 & 2,042 & 11,030 & 2,305 & 8,725 & 2.51 & 30.00 \\
\hline 1936 & $10,711^{*} \neq$ & 2,042 & 12,753 & 1,567 & 11,186 & 1,309 & 9,877 & 2.66 & 30.00 \\
\hline 1937 & $13,248 \neq$ & 1,567 & 14,815 & 6,012 & 8,803 & 1.271 & 7,532 & 2.96 & 43.00 \\
\hline 1938 & $9,822 \neq$ & 6,012 & 15,834 & 3,006 & 12,828 & 2,160 & 10,668 & 2.30 & 7.50 \\
\hline 1939 & $11,462 \neq$ & 3,006 & 14.468 & 2,964 & 11,504 & 1,953 & 9,551 & 2.44 & 20.00 \\
\hline 1940 & $10,742 \neq$ & 2,964 & 13,706 & 953 & 12,753 & 87 & 12,666 & $2.30 \varnothing$ & 20.008 \\
\hline 1941 & $12,733 \neq$ & 953 & 13,686 & & & & & & $48.00 \delta$ \\
\hline
\end{tabular}

* Includes both freestones and clingstones.

† Pack and totál shipments include some, but exports exclude all, peaches repacked into salad and cocktail.

\# Excludes pickled clingstones, in cases: 1936, 115,619; 1937, 111,280; 1938, 24,944; 1939, 195,681; 1940, 156,665; and $1941,187,014$ Preliminary estimate.

Sources of data: Compiled by Giannini Foundation of Agricultural Economics, University of California.

Cols. 1, 2, and 4: Based uponmmeographed releases of Canners League of California.

Col. 8: Weighted averagenthly Summary of Foreign Commerce of the United States, converted at 45 pounds per case. discounts, as compiled by H. R. Well of all grades and sizes of cans on an unadvertised basis and excluding trade

Col. 9: The usual or "going" price generally paid growers by canners for No. 1 canning
allowance for term contracts or sales of lower grades or sales for other uses than canning. 


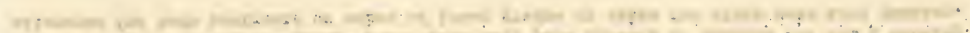

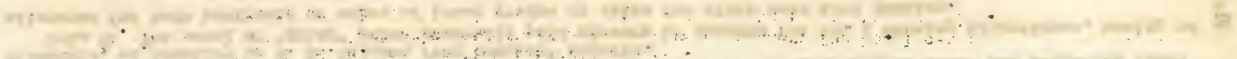

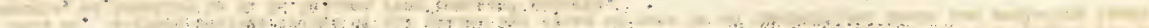

a

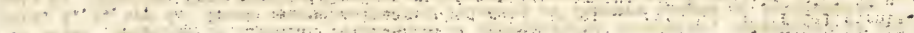

(3)

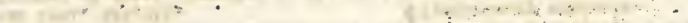

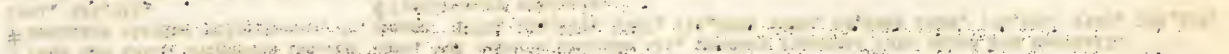

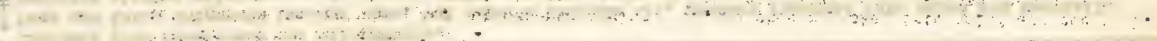

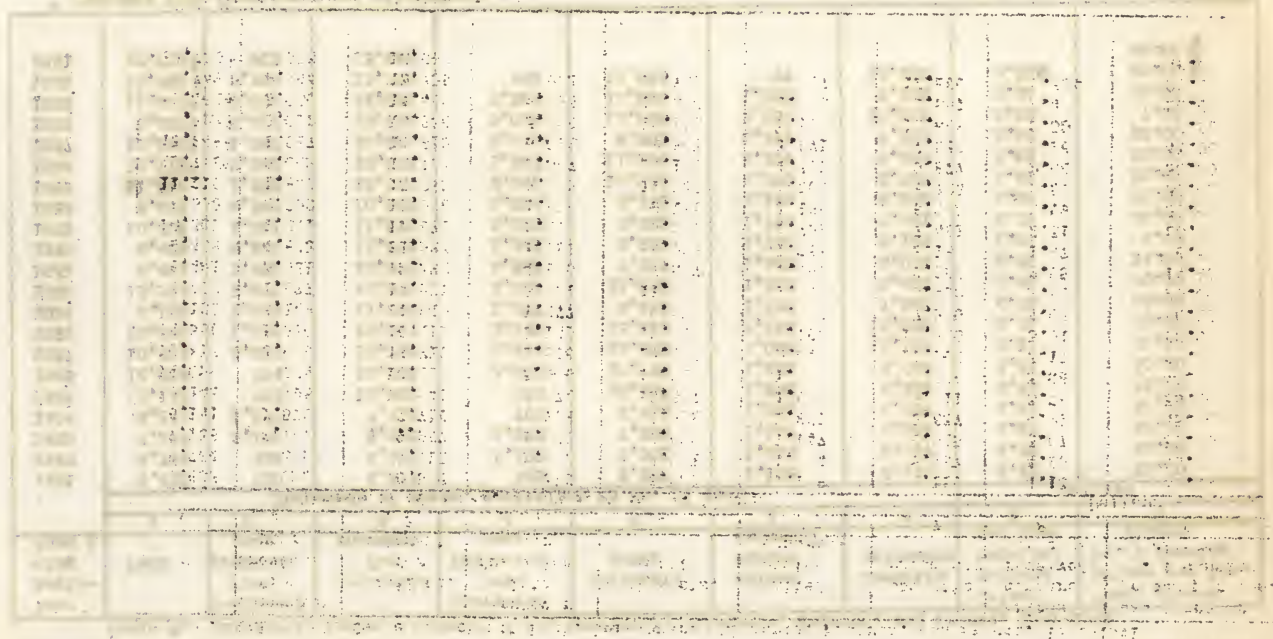




\section{Table 9}

California Canned Peaches: Pack, Carryover, and Movement, 1931-1941

\begin{tabular}{|c|c|c|c|c|c|c|c|c|c|c|c|}
\hline \multirow{3}{*}{$\begin{array}{l}\text { Year } \\
\text { begin- } \\
\text { ning } \\
\text { June } 1\end{array}$} & \multicolumn{3}{|c|}{ Pack } & \multirow{3}{*}{$\begin{array}{l}\text { Supp1y } \\
\text { June } 1\end{array}$} & \multicolumn{3}{|c|}{$\begin{array}{l}\text { Carryover, unshipped } \\
\text { (i.e. sold and unsold) }\end{array}$} & \multicolumn{4}{|c|}{ Movement } \\
\hline & \multirow[b]{2}{*}{ Freestones } & \multirow[b]{2}{*}{ Clingstones } & \multirow[b]{2}{*}{ Total } & & \multirow[b]{2}{*}{$\begin{array}{l}\text { June } 1 \\
\text { of year } \\
\text { indi- } \\
\text { cated }\end{array}$} & \multirow[b]{2}{*}{$\begin{array}{l}\text { De cem- } \\
\text { ber } 31\end{array}$} & \multirow[b]{2}{*}{$\begin{array}{l}\text { June } 1 \\
\text { of fol- } \\
\text { lowing } \\
\text { year }\end{array}$} & \multicolumn{2}{|c|}{ June-December } & \multirow[b]{2}{*}{$\begin{array}{c}\text { January- } \\
\text { May }\end{array}$} & \multirow[b]{2}{*}{$\begin{array}{l}\text { Season's } \\
\text { total } \\
\text { June-May }\end{array}$} \\
\hline & & & & & & & & $\begin{array}{l}\text { Per cent } \\
\text { of } \\
\text { season's } \\
\text { total }\end{array}$ & Quantity & & \\
\hline \multirow{4}{*}{$\begin{array}{l}1931 \\
1932\end{array}$} & 1 & 2 & 3 & 4 & 5 & 6 & 7 & 8 & 9 & 10 & 11 \\
\hline & \multicolumn{7}{|c|}{ Thousands of cases, equivalent 24 No. $2 \frac{1}{2}$ cans } & Per cent & \multicolumn{3}{|c|}{ Thousands of cases } \\
\hline & 72 & 8,349 & 8,421 & 12,372 & 3,951 & 7,151 & 4,845 & 69 & 5,221 & 2,306 & 7,527 \\
\hline & 24 & 6,414 & 6,438 & 11,283 & 4,845 & 4,503 & 1,361 & 68 & 6,780 & 3,142 & 9,922 \\
\hline 1933 & 65 & 10,244 & 10,309 & 11,670 & 1,361 & 4,305 & 2,390 & 79 & 7,365 & 1,915 & 9,280 \\
\hline 1934 & 340 & 8,258 & 8,598 & 10,988 & 2,390 & 3,826 & 1,856 & 78 & 7,162 & 1,970 & 9,132 \\
\hline 1935 & 366 & 10,850 & 11,216 & 13,072 & 1,856 & 6,699 & 2,042 & 58 & 6,373 & 4,657 & 11,030 \\
\hline 1936 & 475 & $10,236^{*}$ & $10,711 *$ & 12,753 & 2,042 & 5,051 & 1,567 & 69 & 7.702 & 3,484 & 11,186 \\
\hline 1937 & 1,043 & $12,205^{*}$ & $13,248^{*}$ & 14,815 & 1,567 & 8,315 & 6,012 & 74 & 6,500 & 2,303 & $8,8 C 3$ \\
\hline 1938 & 376 & $9,446^{*}$ & $9,822^{*}$ & 15,834 & 6,012 & 7,457 & 3,006 & 65 & 8,377 & 4,451 & 12,828 \\
\hline 1939 & 883 & $10,579^{*}$ & $11,462^{*}$ & 14,468 & 3,006 & 6,441 & 2,964 & 70 & 8,027 & 3,477 & 11,504 \\
\hline 1940 & 1,134 & $9,608^{*}$ & $10,742^{*}$ & $.13,706$ & 2,964 & 5,304 & 953 & 66 & 8,402 & 4,351 & 12,753 \\
\hline 1941 & 2,152 & $10,581^{*}$ & $12,733^{*}$ & 13,686 & 953 & $4,294+$ & & & $9,392^{\dagger}$ & & \\
\hline
\end{tabular}

* Excludes pickled clingstone peaches, in cases: 1936, 115,619; 1937, 111,280; 1938, 24,944; 1939, 195,681; $1940,156,665$; and 1941, 187, 014.

f As the stocks in hand December 31, 1941, exclude goods sold to the government, the movement for June - December 1941 includes sales to the government moved and not moved, and so the stocks are relatively too low and the movement too high, as compared with data for other years.

Source of data: Compiled by S. W. Shear, Giannini Foundation of Agricultural Economies, University of California, largely on the basis of data from mimeographed releases of the Canners League of California. 


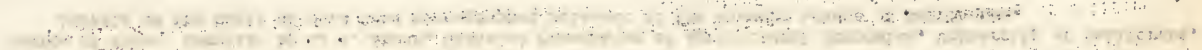

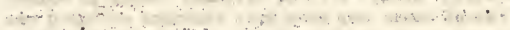

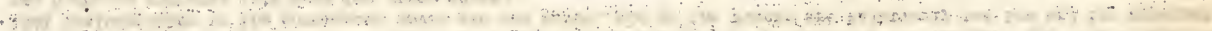

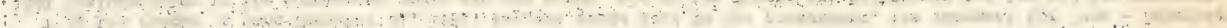

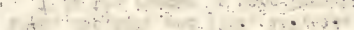

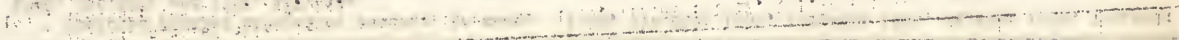

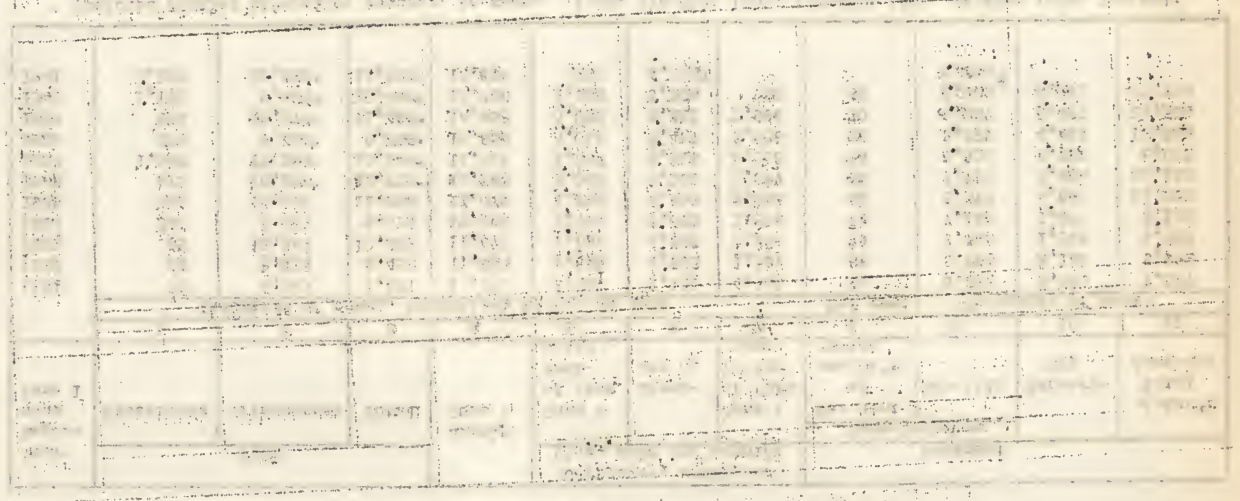

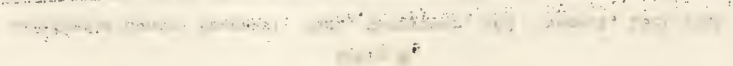


University of California, College of Agriculture

Agricultural Experiment Station, Berkeley, January, 1942

DECIDUOUS FRUIT STATISTICS

PFARS

Table 1

California Pears: Bearing Acreage, Production, Condition, Yield and Farm Value, 1919-1941

\begin{tabular}{|c|c|c|c|c|c|c|}
\hline \multirow[b]{2}{*}{$\begin{array}{l}\text { Crop } \\
\text { year }\end{array}$} & \multirow[b]{2}{*}{$\begin{array}{l}\text { Bearing } \\
\text { acreage }\end{array}$} & \multicolumn{2}{|c|}{ Production } & \multirow[b]{2}{*}{$\begin{array}{l}\text { Yield per } \\
\text { bearing } \\
\text { acre }\end{array}$} & \multicolumn{2}{|c|}{ Farm* value of crop } \\
\hline & & Total & $\begin{array}{l}\text { November } 1 \\
\text { per cent of } \\
\text { full crop }\end{array}$ & & $\begin{array}{l}\text { Price to } \\
\text { growers } \\
\text { per ton }\end{array}$ & $\begin{array}{l}\text { Total } \\
\text { returns } \\
\text { to growers }\end{array}$ \\
\hline & 1 & 2 & 3 & 4 & 5 & 6 \\
\hline & Acres & Short tons & Per cent & Short tons & Dollars & $\overline{1}, \overline{000}$ dollars \\
\hline 1919 & 23,100 & 111,000 & 98 & 4.8 & 72 & 7,992 \\
\hline 1920 & 29,400 & 105,000 & 85 & 3.6 & 90 . & 9,450 \\
\hline 1921 & 31,400 & 89,000 & 63 & 2.8 & 62 & 5,518 \\
\hline 1922 & 37,700 & 150,000 & 96 & 4.0 & 50 & 7,500 \\
\hline 1923 & 41,000 & 136,000 & 90 & 3.3 & 50 & 6,800 \\
\hline 1924 & 43,100 & 133,000 & 73 & 3.1 & 66 & 8,778 \\
\hline 1925 & 46,800 & 181,000 & 85 & 3.9 & 52 & 9,412 \\
\hline 1926 & 52,000 & 204,000 & 90 & 3.9 & 35 & 7,140 \\
\hline 1927 & 57,600 & $181,000+$ & 69 & 3.1 & 54 & 9,666 \\
\hline 1928 & 60,700 & $226,000 \dagger$ & 86 & 3.7 & 37 & 8,288 \\
\hline 1929 & 65,600 & 190,000 & 68 & 2.9 & 69 & 13,110 \\
\hline 1930 & 65,300 & $273,000 t$ & 84 & 4.2 & 23 & 5,566 \\
\hline 1931 & 66,400 & $217,000+$ & 67 & 3.3 & 24 & 4,848 \\
\hline 1932 & 69,600 & $244,000+$ & 70 & 3.5 & 12 & 2,160 \\
\hline 1933 & 70,500 & $221,000+$ & 65 & 3.1 & 21 & 3,801 \\
\hline 1934 & 69,000 & $233,000+$ & 68 & 3.4 & 32 & 7,168 \\
\hline 1935 & 61,000 & 163,000 & 49 & 2.7 & 28 & 4,564 \\
\hline 1936 & $53,800 \neq$ & 240,000 & 70 & 4.5 & 27 & 6,480 \\
\hline 1937 & $53,800 \neq$ & $224,000 t$ & 71 & 4.2 & 28 & 5,936 \\
\hline 1938 & $52,800 \neq$ & $282,000 t$ & 83 & 5.3 & 14 & 3,640 \\
\hline 1939 & $51,100 \ddagger$ & $253,000+$ & 74 & 5.0 & 26 & 6,448 \\
\hline 1940 & $47,000 \neq$ & $226,000+$ & 70 & 4.8 & 26 & 5,642 \\
\hline 19419 & 45,700 & 223,000 & 70 & 4.9 & 40 & 8,920 \\
\hline & & & & & & \\
\hline
\end{tabular}

point.

* Growers' returns for naked fruit delivered at growers' first delivery

† Includes unharvested tonnage: $1927,2,000 ; 1928,2,000 ; 1930,31,000$; $1931,15,000 ; 1932,64,000 ; 1933,40,000 ; 1934,9,000 ; 1937,12,000 ; 1938$, 22,$000 ; 1939,5,000$; and $1940,9,000$.

F Nonbearing acres: $1936,5,700 ; 1937,4,700 ; 1938,3,700 ; 1939,2,900$; and $1940,2,200$.

\& Preliminary estimates.

Sources of data:

Compiled by S. W. Shear, Giannini Foundation of Agricultural Economics, University of California, from United States and California Crop Keports, except cols. 4 and 6 calculated. 


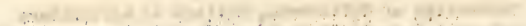

$\because \therefore \therefore \div$

. $.2 \ldots$

a. . . .

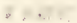

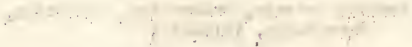

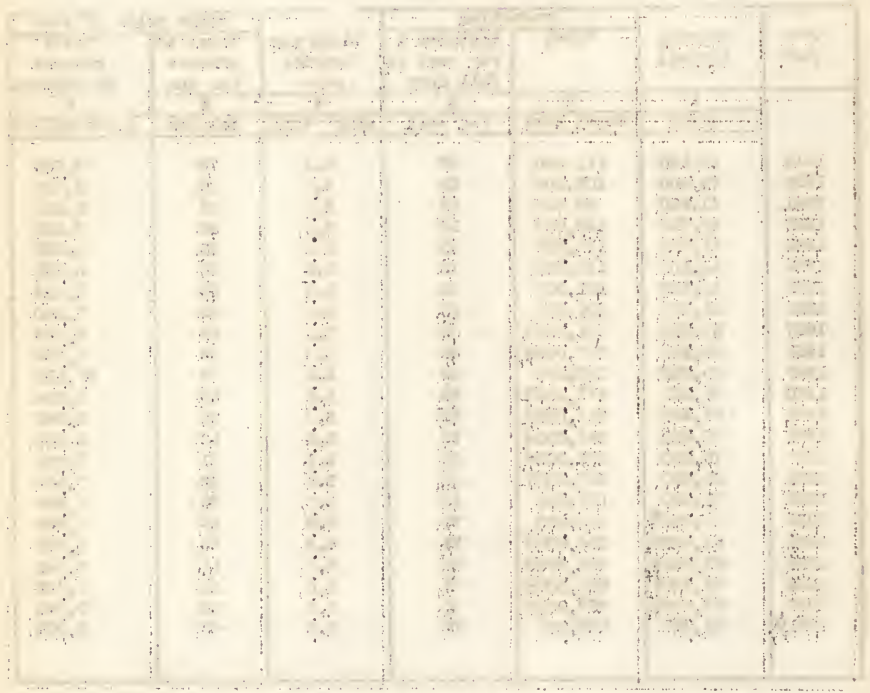

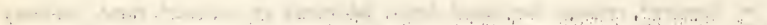

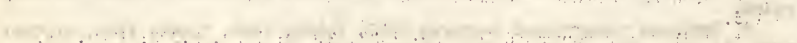

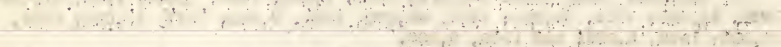

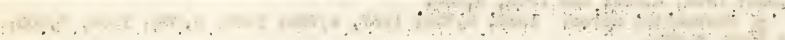
$\therefore+\cdots+\cdots$ (1...

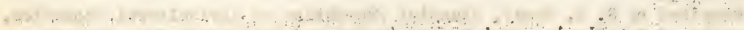

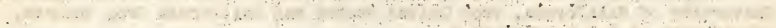

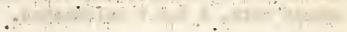


Table 2. California Bartletts and Other Pears: Bearing Acreage, Production, Condition, Yield, and Farm Value, 1927-1941

\begin{tabular}{|c|c|c|c|c|c|c|}
\hline \multirow[b]{2}{*}{$\begin{array}{l}\text { Crop } \\
\text { year }\end{array}$} & \multirow[b]{2}{*}{$\begin{array}{l}\text { Bearing } \\
\text { acreage }\end{array}$} & \multicolumn{2}{|c|}{ Production } & \multirow[b]{2}{*}{$\begin{array}{l}\text { Yield per } \\
\text { bearing } \\
\text { acre }\end{array}$} & \multirow{2}{*}{\multicolumn{2}{|c|}{$\begin{array}{ll}\text { Farm* value of crop } \\
\text { Price to } & \text { Total } \\
\text { growers } & \text { returnst } \\
\text { per ton } & \text { to growers }\end{array}$}} \\
\hline & & Total & $\begin{array}{l}\text { November I } \\
\text { per cent of } \\
\text { full crop }\end{array}$ & & & \\
\hline \multirow[b]{4}{*}{1927} & 1 & 2 & 3 & 4 & 5 & 6 \\
\hline & Acres & Short tons & Per cent & short tons & Dollars & 1,000 dollars \\
\hline & \multicolumn{6}{|c|}{ Bartletts } \\
\hline & 51,700 & $161,200 \neq$ & $-\xi$ & 3.1 & $-\infty$ & - \\
\hline 1928 & 54,100 & $203,000 \neq$ & -- & 3.8 & - & -- \\
\hline 1929 & 57,300 & 171,000 & -- & 3.0 & -- & - \\
\hline 1930 & 56,500 & $241,000 \neq$ & - & 4.3 & - & $-\infty$ \\
\hline 1931 & 57,200 & $195,000 \neq$ & - & 3.4 & - & -- \\
\hline 1932 & 59,900 & $217,000 \neq$ & -- & 3.6 & -- & -- \\
\hline 1933 & 60,500 & $193,000 \neq$ & - & 3.2 & -- & -- \\
\hline 1934 & 59,000 & $207,000 \neq$ & -- & 3.5 & - & - \\
\hline 1935 & 52,600 & 146,000 & - & 2.8 & 28 & 4,088 \\
\hline 1936 & 45,500 & 214,000 & -- & 4.7 & 27 & 5,778 \\
\hline 1937 & 45,500 & $202,000 \neq$ & -- & 4.4 & 28 & 5,376 \\
\hline 1938 & 44,500 & $234,000 \neq$ & -- & 5.3 & 14 & 2,996 \\
\hline 1939 & 43,300 & $221,000 \neq$ & 74 & 5.1 & 28 & 6,132 \\
\hline 1940 & $39,800 \$$ & $190,000 \neq$ & 69 & 4.8 & 27 & 4,995 \\
\hline $1941 \|$ & 39,300 & 204,000 & 74 & 5.2 & 41 & 8,364 \\
\hline \multirow{2}{*}{\multicolumn{7}{|c|}{19,800}} \\
\hline & 5,900 & 19,800 & - & 3.4 & - & - \\
\hline 1928 & 6,600 & 23,000 & -- & 3.5 & -- & -- \\
\hline 1929 & 8,300 & 19,000 & - & 2.3 & -- & -- \\
\hline 1930 & 8,800 & $32,000 \neq$ & -- & 3.6 & -- & -- \\
\hline 1931 & 9,200 & 22,000 & -- & 2.4 & -- & $-\infty$ \\
\hline 1932 & 9,700 & $27,000 \neq$ & - & 2.8 & -- & - \\
\hline 1933 & 10,000 & $28,000 \neq$ & -- & 2.8 & - & -- \\
\hline 1934 & 10,000 & $26,000 \neq$ & -- & 2.6 & - & - \\
\hline 1935 & 8,400 & 17,000 & -- & 2.0 & 29 & 493 \\
\hline 1936 & 8,300 & 26,000 & -- & 3.1 & 27 & 702 \\
\hline 1937 & 8,300 & $22,000 \neq$ & - & 2.7 & 29 & 580 \\
\hline 1938 & 8,300 & $48,000 \neq$ & - & 5.8 & 11 & 506 \\
\hline 1939 & 7,800 & $32,000 \neq$ & 72 & 4.1 & 13 & 377 \\
\hline 1940 & 7,2009 & $36,000 \neq$ & 79 & 5.0 & 17 & 544 \\
\hline $1941 \|$ & 6,400 & 19,000 & 46 & 3.0 & 38 & 722 \\
\hline
\end{tabular}

point.

Growers ${ }^{\prime}$ retums for naked fruit delivered at growers ${ }^{\prime}$ first delivery

t Sum of returns from Bartletts and other varieties in this column does not equal value given in table $1, \operatorname{col} .6$, because rounded prices per ton used in col. 5 .

\# Includes unharvested production show in col. 2 of tables 3 and 4 .

$\oint$ Dashes indicate data not available.

$\$$ Nonbearing acreage in 1940 was 1,600 of Bartletts and 600 of other varieties.

\| Preliminary.

Sources of data:

Compiled by S. W. Shear, Giannini Foundation of Agricultural Fcononics,

University of California, from United States and California Crop Reparts,

except cols. 4 and 6 cal culated. 

Table 3

California Bartlett Pears: Production and Utilization, 1919-1941

\begin{tabular}{|c|c|c|c|c|c|c|c|}
\hline \multirow[b]{2}{*}{$\begin{array}{l}\text { Crop } \\
\text { year }\end{array}$} & \multicolumn{3}{|c|}{ Production } & \multirow[b]{2}{*}{ Canned } & \multirow[b]{2}{*}{$\begin{array}{l}\text { Dried, } \\
\text { fresh } \\
\text { weight }\end{array}$} & \multicolumn{2}{|c|}{ Used fresh } \\
\hline & Total & $\begin{array}{c}\text { Un- } \\
\text { harvested }\end{array}$ & Harvested & & & $\begin{array}{c}\text { Shipped } \\
\text { fresh } \\
\text { out of } \\
\text { state }\end{array}$ & $\begin{array}{l}\text { Used } \\
\text { fresh } \\
\text { vithin } \\
\text { state }\end{array}$ \\
\hline \multirow{3}{*}{ Averages: } & 1 & 2 & 3 & 4 & 5 & 6 & 7 \\
\hline & \multicolumn{7}{|c|}{ Short tons, fresh equival ent weight } \\
\hline & 111,000 & 0 & 111,000 & 32,400 & 17,000 & 54,700 & 6,900 \\
\hline $1925-1929$ & 176,900 & 800 & 176,100 & 55,000 & 23,100 & 85,800 & 12,200 \\
\hline $1930-1934$ & 210,600 & 28,800 & 181,800 & 51,000 & 28,800 & 80,400 & 21,600 \\
\hline $1935-1939$ & 203,400 & 6,400 & 197,000 & 68,500 & 35,400 & 70,700 & 22,400 \\
\hline \multicolumn{8}{|l|}{ Annual: } \\
\hline 1919 & 104,600 & 0 & 104,600 & 28,200 & 24,800 & 45,000 & 6,600 \\
\hline 1920 & 99,000 & 0 & 99,000 & 31,200 & 14,900 & 47,000 & 5,900 \\
\hline 1921 & 83,600 & 0 & 83,600 & 23,000 & 6,600 & 47,600 & 6,400 \\
\hline 1922 & 137,500 & 0 & 137,500 & 45,000 & 27,500 & 57,800 & 7,200 \\
\hline 1923 & 122,600 & 0 & 122,600 & 30,000 & 11,000 & 74,500 & 7,100 \\
\hline 1924 & 119,000 & 0 & 119,000 & 37,000 & 17,300 & 56,400 & 8,300 \\
\hline 1925 & 163,600 & 0 & 163,600 & 58,000 & 19,300 & 76,900 & 9,400 \\
\hline 1926 & 185,600 & 0 & 185,600 & 51,000 & 23,400 & 101,300 & 9,900 \\
\hline 1927 & 161,200 & 2,000 & 159,200 & 49,000 & 19,000 & 79,200 & 12,000 \\
\hline 1928 & 203,000 & 2,000 & 201,000 & 61,500 & 30,600 & 93,600 & 15,300 \\
\hline 1929 & 171,000 & 0 & 171,000 & 55,300 & 23,100 & 78,100 & 14,500 \\
\hline 1930 & 241,000 & 30,000 & 211,000 & 49,300 & 24,800 & 116,100 & 20,800 \\
\hline 1931 & 195,000 & 15,000 & 180,000 & 47,600 & 24,000 & 87,500 & 20,900 \\
\hline 1932 & 217,000 & 60,000 & 157,000 & 37,300 & 30,000 & 67,200 & 22,500 \\
\hline 1933 & 193,000 & 33,000 & 160,000 & 50,700 & 38,300 & 52,100 & 18,900 \\
\hline 1934 & 207,000 & 6,000 & 201,000 & 70,100 & 27,100 & 79,100 & 24,700 \\
\hline 1935 & 146,000 & 0 & 146,000 & 36,000 & 33,600 & 54,200 & 22,200 \\
\hline 1936 & 214,000 & 0 & 214,000 & 74,500 & 44,400 & 72,100 & 23,000 \\
\hline 1937 & 202,000 & 10,000 & 192,000 & 76,100 & 19,200 & 78,800 & 17,900 \\
\hline 1938 & 234,000 & 20,000 & 214,000 & 70,500 & 35,400 & 82,900 & 25,200 \\
\hline 1939 & 221,000 & 2,000 & 219,000 & 85,600 & 44,400 & 65,500 & 23,500 \\
\hline 1940 & 190,000 & 5,000 & 185,000 & 86,300 & 16,900 & 62,200 & 19,600 \\
\hline $1941 *$ & 204,000 & 0 & 204,000 & 113,700 & 21,400 & 48,000 & 20,900 \\
\hline
\end{tabular}

* Data for 1941 are preliminary and utilization figures are unofficial estimates.

Sources of data:

Compiled by S. W. Shear, Giamini Foundation of Agricultural poonomics, University of California, largely from data of the United States and the Cal ifornia Crop Reporting Services. 
Table 4

California Production and Utilization* of Pears other than Bartletts and Fresh Use of All Pears, 1919-1941

\begin{tabular}{|c|c|c|c|c|c|c|c|}
\hline \multirow{3}{*}{$\begin{array}{l}\text { Crop } \\
\text { year }\end{array}$} & \multicolumn{5}{|c|}{ Other than Bartletts } & \multicolumn{2}{|c|}{$\begin{array}{l}\text { All varieties, } \\
\text { used fresh }\end{array}$} \\
\hline & \multicolumn{3}{|c|}{ Production } & \multicolumn{2}{|c|}{ Used fresh } & \multirow[b]{2}{*}{$\begin{array}{l}\text { Shipped } \\
\text { fresh } \\
\text { out of } \\
\text { state }\end{array}$} & \multirow[b]{2}{*}{$\begin{array}{l}\text { Used } \\
\text { fresh } \\
\text { within } \\
\text { state }\end{array}$} \\
\hline & Total & $\begin{array}{c}\text { Un- } \\
\text { harvested }\end{array}$ & Harvested* & $\begin{array}{l}\text { Shipped } \\
\text { fresh } \\
\text { out of } \\
\text { state }\end{array}$ & $\begin{array}{l}\text { Used } \\
\text { fresh } \\
\text { within } \\
\text { state }\end{array}$ & & \\
\hline \multirow{6}{*}{$\begin{array}{l}\text { Averages: } \\
1919-1924 \\
1925-1929 \\
1930-1934 \\
1935-1939\end{array}$} & I & 2 & 3 & 4 & 5 & 6 & 7 \\
\hline & \multicolumn{7}{|c|}{ Short tons, fresh equivalent weight } \\
\hline & 9,600 & 0 & 9,600 & 9,100 & 500 & 63,800 & 7,400 \\
\hline & 19,500 & 0 & 19,500 & 18,100 & 1,400 & 103,900 & 13,600 \\
\hline & 27,000 & 3,000 & 24,000 & 21,100 & 2,900 & 101,500 & 24,500 \\
\hline & 29,000 & 1,400 & $27,600 *$ & 23,300 & 4,000 & 94,000 & 26,400 \\
\hline \multicolumn{8}{|l|}{ Annual: } \\
\hline 1919 & 6,400 & 0 & 6,400 & 6,000 & 400 & 51,000 & 7,000 \\
\hline 1920 & 6,000 & 0 & 6,000 & 5,700 & 300 & 52,700 & 6,200 \\
\hline 1921 & 5,400 & 0 & 5,400 & 5,000 & 400 & 52,600 & 6,800 \\
\hline 1922 & 12,500 & 0 & 12,500 & 12,000 & 500 & 69,800 & 7,700 \\
\hline 1923 & 13,400 & 0 & 13,400 & 12,800 & 600 & 87,300 & 7,700 \\
\hline 1924 & 14,000 & 0 & 14,000 & 13,200 & 800 & 69,600 & 9,100 \\
\hline 1925 & 17,400 & 0 & 17,400 & 16,500 & 900 & 93,400 & 10,300 \\
\hline 1926 & 18,400 & 0 & 18,400 & 17,400 & 1,000 & 118,700 & 10,900 \\
\hline 1927 & 19,800 & 0 & 19,800 & 18,600 & 1,200 & 97,800 & 13,200 \\
\hline 1928 & 23,000 & 0 & 23,000 & 21,600 & 1,400 & 115,200 & 16,700 \\
\hline 1929 & 19,000 & 0 & 19,000 & 16,700 & 2,300 & 94,800 & 16,800 \\
\hline 1930 & 32,000 & 1,000 & 31,000 & 27,600 & 3,400 & 143,700 & 24,200 \\
\hline 1931 & 22,000 & 0 & 22,000 & 19,100 & 2,900 & 106,600 & 23,800 \\
\hline 1932 & 27,000 & 4,000 & 23,000 & 20,200 & 2,800 & 87,400 & 25,300 \\
\hline 1933 & 28,000 & 7,000 & 21,000 & 18,800 & 2,200 & 70,900 & 21,100 \\
\hline 1934 & 26,000 & 3,000 & 23,000 & 19,900 & 3,100 & 99,000 & 27,800 \\
\hline 1935 & 17,000 & 0 & $17,000 *$ & 14,000 & 2,600 & 68,200 & 24,800 \\
\hline 1936 & 26,000 & 0 & $26,000 *$ & 21,900 & 3,400 & 94,000 & 26,400 \\
\hline 1937 & 22,000 & 2,000 & $20,000 *$ & 16,300 & 3,200 & 95,100 & 21,100 \\
\hline 1938 & 48,000 & 2,000 & 46,000 & 41,700 & 4,300 & 124,600 & 29,500 \\
\hline 1939 & 32,000 & 3,000 & 29,000 & 22,300 & 6,700 & 87,800 & 30,200 \\
\hline 1940 & 36,000 & 4,000 & $32,000 *$ & 14,700 & 6,800 & 76,900 & 26,400 \\
\hline $1941+$ & 19,000 & 0 & $19,000 *$ & 5,000 & 3,200 & 53,000 & 24,100 \\
\hline
\end{tabular}

* Includes 200 fresh tons dried in 1936 and 100 in 1940, and 400 tons canned in 1935,500 in 1936 and 1937, 10,400 in 1940, and 10,800 in 1941 .

+ Data for 1941 are preliminary and utilization figures are unofficial estimates.

Sources of data:

Compiled by S. W. Shear, Giannini Foundation of Agricultural Economics,

University of California, largely from data of the United States and the

California Crop Reporting Services. 


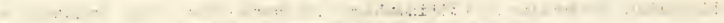
$\therefore \quad \therefore \quad \therefore \quad+\cdots+\cdots+\cdots$

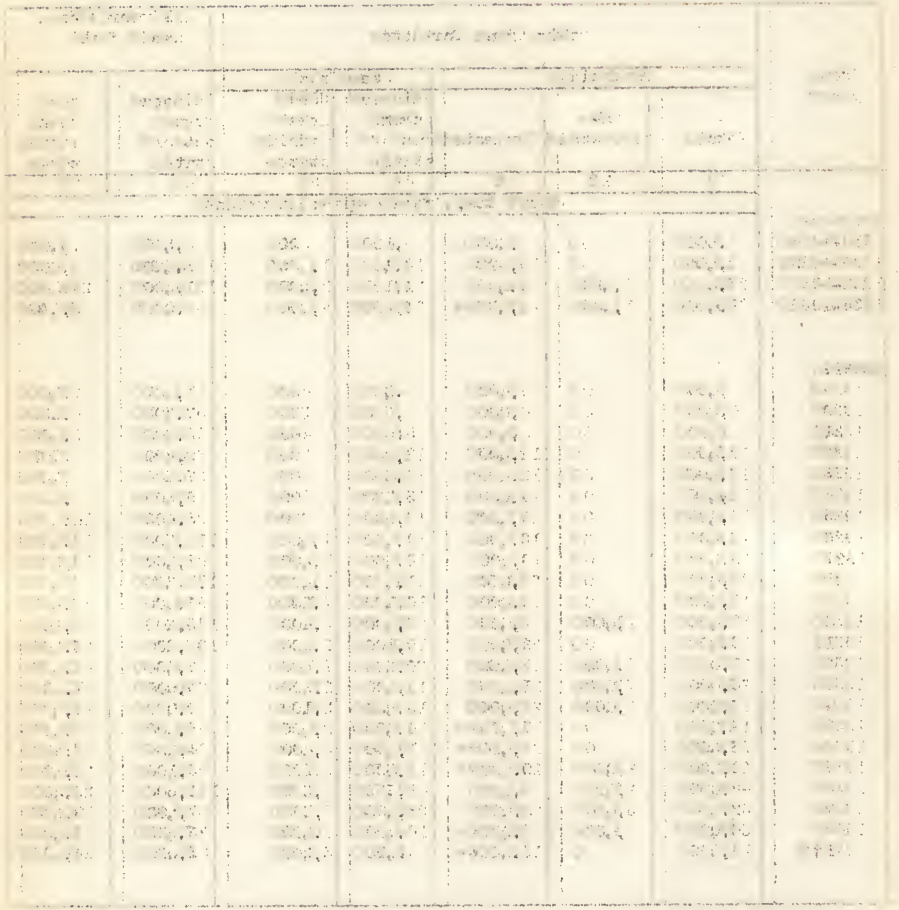

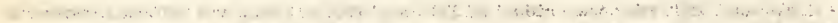

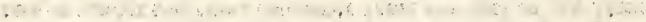

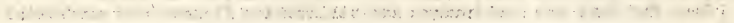


Table 5

Pacific Coast Bartlett Pear Production,* 1925-1941

\begin{tabular}{|c|c|c|c|c|c|}
\hline \multirow{2}{*}{ Crop year } & \multirow{2}{*}{$\begin{array}{c}\text { Pacific } \\
\text { Coast }\end{array}$} & \multirow{2}{*}{ Californiat } & \multicolumn{3}{|c|}{ Pacific Horthwest } \\
\hline & & & Total & Oregon & Washington \\
\hline \multirow[b]{3}{*}{ Averages: } & 1 & 2 & 3 & 4 & 5 \\
\hline & \multicolumn{5}{|c|}{ Short tons } \\
\hline & & 376.880 & 90760 & & \\
\hline \begin{tabular}{|l|}
$1925-1929$ \\
$1930-1934$
\end{tabular} & $\begin{array}{l}267,040 \\
324,330\end{array}$ & $\begin{array}{l}176,880 \\
210,600\end{array}$ & $\begin{array}{r}90,160 \\
113,730\end{array}$ & $\begin{array}{l}29,310 \\
32,170\end{array}$ & 60,850 \\
\hline $\begin{array}{l}1930-1934 \\
1935-1939\end{array}$ & 337,450 & 203,400 & 134,050 & 36,520 & 97,530 \\
\hline & & & & & 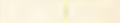 \\
\hline \multicolumn{6}{|l|}{ Annual: } \\
\hline 1925 & 241,630 & 163,600 & 78,030 & 25,580 & 52,450 \\
\hline 1926 & 291,030 & 185,600 & 105,430 & 28,380 & 77,050 \\
\hline 1927 & $221,950 *$ & $161,200 *$ & 60,750 & 21,250 & 39,500 \\
\hline 1928 & $308,930 *$ & $203,000 *$ & 105,930 & 37,850 & 68,080 \\
\hline 1929 & 271,680 & 171,000 & 100,680 & 33,500 & 67,180 \\
\hline 1930 & $363,420 *$ & $241,000 *$ & 122,420 & 36,220 & 86,200 \\
\hline 1931 & $292,680 *$ & $195,000 *$ & 97,680 & 30,000 & 67,680 \\
\hline 1932 & $326,260 *$ & $217,000 *$ & $109,260 *$ & 33,080 & $76,180 *$ \\
\hline 1933 & $313,300 *$ & $193,000 *$ & 120,300 & 29,450 & 90,850 \\
\hline 1934 & $325,980 *$ & $207,000 *$ & 118,980 & 32,080 & 86,900 \\
\hline 1935 & 275,450 & 146,000 & 129,450 & 36,250 & 93,200 \\
\hline 1936 & 356,000 & 214,000 & 142,000 & 42,000 & 100,000 \\
\hline 1937 & $323,370 *$ & $202,000 *$ & $121,370 *$ & $27,950 *$ & $93,420 *$ \\
\hline 1938 & $378,420 *$ & $234,000 *$ & $144,420 *$ & $35,920 *$ & $108,500 *$ \\
\hline 1939 & $354,000 *$ & $221,000 *$ & $133,000 *$ & $40,500 *$ & $92,500 *$ \\
\hline 1940 & $327,250 *$ & $190,000 *$ & $137,250 *$ & 42,250 & $95,000 *$ \\
\hline $1941 \ddagger$ & 343,200 & 204,000 & 139,200 & 43,580 & 95,620 \\
\hline & & & & & \\
\hline
\end{tabular}

* Includes unharvested tonnages: California, 1927, 2,000; 1928, 2,000; $1930,30,000 ; 1931,15,000 ; 1932,60, \overline{000 ; 1933}, 33,000 ; 1934,6,000 ; 1937$, 10,$000 ; 1938,20,000 ; 1939,2,000 ; 1940,5,000$. Washington, $1932,5,000$; $1937,2,100$; 1938, 25,420; 1939, 6,450; 1940, 3,850. Oregon, 1937, 1,200; $1938,5,750 ; 1939,2,020$.

+Average California Bartlett production in 1919-1924 was 111,000 tons; see table 3 for annual data. Estimates of Northwest Bartletts unavailable before 1925.

$\neq$ Preliminary.

Sources of data:

Compiled by S. W. Shear, Giannini Foundation of Agricultural. Fconomios, University of California. 1925-1938 from U. S. Dept. Agr. Crop Reporting Board, General Crop Report, December 1939, pp. 41 and 84 , December 19, 1939 (Mimeo.), washington, D.C., except 1938 unharvested production from U. S. Dept. Agr., Agrioultural Statistics, 1940, p. 247. 1939-1941 from recent United States Crop Reports. Oregon and "ashington data converted to tons at 50 pounds per bushel, and Califarnia at 48 pounds per bushel. 

Table 6

Pacific Coast Production* of Pears Other than Bartletts, 1925-1941

\begin{tabular}{|c|c|c|c|c|c|}
\hline \multirow{2}{*}{ Crop year } & \multirow{2}{*}{$\begin{array}{c}\text { Pacific } \\
\text { Coast }\end{array}$} & \multirow{2}{*}{ California $†$} & \multicolumn{3}{|c|}{ Pacific Northwest } \\
\hline & & & Tot al & Oregon & Mashington \\
\hline \multirow[b]{3}{*}{ Averages : } & $I$ & 2 & 3 & 4 & 5 \\
\hline & \multicolumn{5}{|c|}{ Short tons } \\
\hline & & & & & \\
\hline $1925-1929$ & 67,200 & 19,520 & 47,680 & 32,940 & 14,740 \\
\hline 1930-1934 & 91,160 & 27,000 & 64,160 & 36,780 & 27,380 \\
\hline 1935-1939 & 133,160 & 29,000 & 104,160 & 59,290 & 44,870 \\
\hline \multicolumn{6}{|l|}{ Annual: } \\
\hline 1925 & 44,230 & 17,400 & 26,830 & 17,180 & 9,650 \\
\hline 1926 & 66,970 & 18,400 & 48,570 & 35,620 & 12,950 \\
\hline 1927 & 65,700 & 19,800 & 45,900 & 35,000 & 10,900 \\
\hline 1928 & 85,970 & 23,000 & 62,970 & 38,650 & 24,320 \\
\hline 1929 & 73,130 & 19,000 & 54,130 & 38,250 & 15,880 \\
\hline 1930 & $112,080 *$ & $32,000 *$ & 80,080 & 48,780 & 31,300 \\
\hline 1931 & 70,180 & 22,000 & 48,180 & 20,000 & 28,180 \\
\hline 1932 & $92,640 *$ & $27,000 *$ & $65,640 *$ & $40,520 *$ & $25,120 *$ \\
\hline 1933 & $95,020 *$ & $28,000 *$ & $67,020 *$ & $41,020 *$ & $26,000 *$ \\
\hline 1934 & $85,880 *$ & $26,000 *$ & 59,880 & 33,580 & 26,300 \\
\hline 1935 & 101,920 & 17,000 & 84,920 & 48,120 & 36,800 \\
\hline 1936 & 113,000 & 26,000 & 87,000 & 52,000 & 35,000 \\
\hline 1937 & $129,380 *$ & $22,000 *$ & $107,380 *$ & $60,800 *$ & $46,580 *$ \\
\hline 1938 & $172,300 *$ & $48,000 *$ & $124,300 *$ & $70,300 *$ & $54,000 *$ \\
\hline 1939 & $149,200 *$ & $32,000 *$ & $117,200 *$ & $65,220 *$ & $51,980 *$ \\
\hline 1940 & $162,380 * \neq$ & $36,000 *$ & $126,380 * \neq$ & $68,880 \neq$ & $57,500 * \neq$ \\
\hline $1941 \S$ & 138,750 & 19,000 & 119,750 & 62,900 & 56,850 \\
\hline
\end{tabular}

* Includes unharvested tonnages: California, 1930, 1,000; 1932, 4,000; $1933,7,000 ; 1934,3,000 ; 1937,2,000 ; 1938,2,000 ; 1939,3,000 ; 1940,4,000$. Oregon, $1932,8,750 ; 1933,7,000 ; 1937,1,750 ; 1938,4,920 ; 1939,2,680$. vashington, 1932, 10,000; 1933, 12,500; 1937, 2,180; 1938, 6,520; 1939, 9,400; $\overline{1940,8,630}$.

+Average California production of pears other than Partletts in 1919-1924 was 9,600 tons; see table 4 for annual data. Istimates not available for the Northwest before 1925.

fIncludes 6,550 tons in Nashington and 2,000 tons in Oregon of pears harvested but not utilized because of grade and size requirements of marketing agreements.

$\oint$ Preliminary.

Sources of data:

Compiled by S. W. Shear, Giannini Foundation of Agricultural Economics,

University of Califomia. $1925-1938$ from U. S. Dept. Agr., Crop Reporting

Board, General Crop Report, December 1939, pp. 41 and 84, Decemher 19, 1939

(Mimeo.), "ashington, D. C., except 1938 unharvested prodiction from U. S.

Dept. Agr., Agricultural Statistics, 1940, p. 247. 1939-1941 from recent

United States Crop Reports. Oregon and ashington data converted to tons at

50 pounds per bushel, and California at 48 pounds per bushel. 
. . $\because: \cdot$

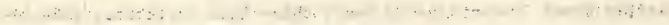

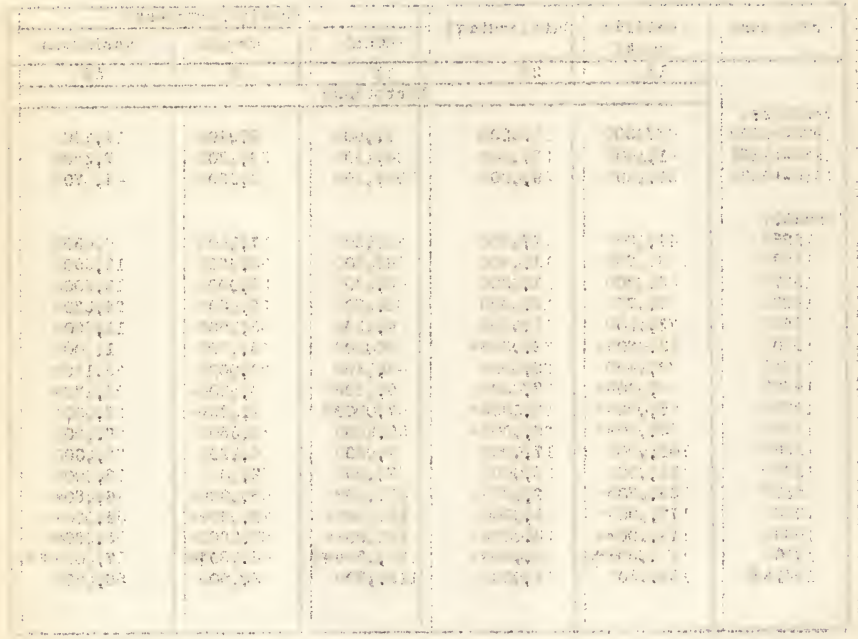

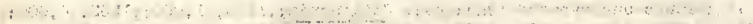

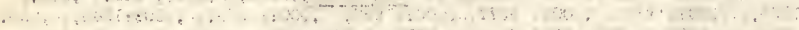

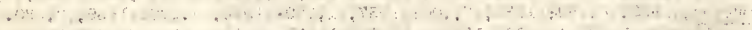

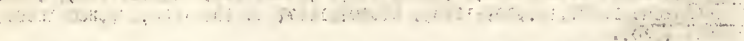

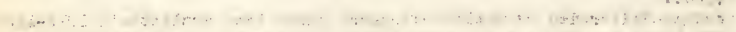

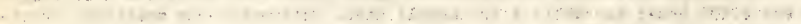
$\ldots+\cdots+\cdots, \cdots$

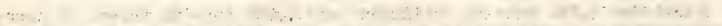

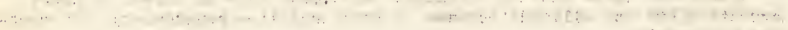
$\therefore+\cdots$ $\ldots+\cdots$

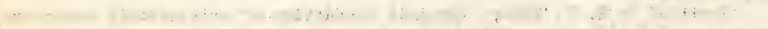

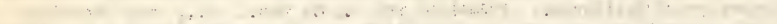

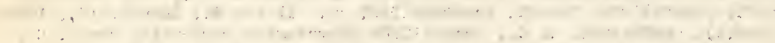
. $\ldots+\ldots, \ldots, \ldots+\ldots+\ldots, \ldots+\ldots$

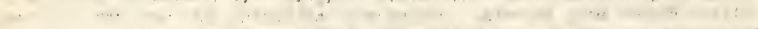

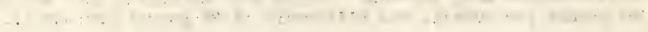


January, 1942

PEARS

Table 7. Pacific Coast Pears: Production and Utilization, 1919-1941

\begin{tabular}{|c|c|c|c|c|c|c|c|c|c|}
\hline \multirow[b]{3}{*}{$\begin{array}{l}\text { Crop } \\
\text { year }\end{array}$} & \multicolumn{4}{|c|}{$\begin{array}{l}\text { Harvested production, } \\
\text { all varieties }\end{array}$} & \multicolumn{5}{|c|}{ Utilization, Pacific Coast } \\
\hline & \multirow[b]{2}{*}{$\begin{array}{l}\text { United } \\
\text { states }\end{array}$} & \multicolumn{3}{|c|}{ Pacific Coast } & \multicolumn{3}{|c|}{ Canned* } & \multirow{2}{*}{$\begin{array}{l}\text { Dried, } \dagger \\
\text { California, } \\
\text { fresh } \\
\text { equiva- } \\
\text { lent }\end{array}$} & \multirow[b]{2}{*}{$\begin{array}{l}\text { other } \\
\text { uses, } \\
\text { mostly } \\
\text { fresh }\end{array}$} \\
\hline & & Total & $\begin{array}{l}\text { Cali- } \\
\text { fornia }\end{array}$ & $\begin{array}{l}\text { Oregon } \\
\text { and } \\
\text { Wash- } \\
\text { ington }\end{array}$ & $\begin{array}{l}\text { Total } \\
\text { Pacific }\end{array}$ & $\begin{array}{r}\text { Cali- } \\
\text { fornia }\end{array}$ & $\begin{array}{l}\text { Oregon } \\
\text { and } \\
\text { iash- } \\
\text { ington }\end{array}$ & & \\
\hline & 1 & 2 & 3 & 4 & 5 & 6 & 7 & 8 & 9 \\
\hline & \multicolumn{8}{|c|}{ Thousands of short tons, fresh equivalent weight } & \\
\hline 1919 & 368 & 173 & 111 & 62 & 38 & 28 & 10 & 25 & 110 \\
\hline 1920 & 431 & 166 & 105 & 61 & 41 & 31 & 10 & 15 & 110 \\
\hline 1921 & 285 & 161 & 89 & 72 & 33 & 23 & 10 & 7 & 121 \\
\hline 1922 & 506 & $\cdot 240$ & 150 & 90 & 66 & 45 & 21 & 28 & 146 \\
\hline 1923 & 427 & 242 & 136 & 106 & 46 & 30 & 16 & 11 & 185 \\
\hline 1924 & 463 & 218 & 133 & 85 & 56 & 37 & 19 & 17 & 145 \\
\hline 1925 & 498 & 286. & 181 & 105 & 90 & 58 & 32 & 19 & 177 \\
\hline 1926 & 613 & 358 & 204 & 154 & 85 & 51 & 34 & 23 & 250 \\
\hline 1927 & 449 & 286 & 179 & 107 & 69 & 49 & 20 & 19 & 193 \\
\hline 1928 & 589 & 393 & 224 & 169 & 112 & 62 & 50 & 31 & 250 \\
\hline 1929 & 532 & 345 & 190 & 155 & 117 & 55 & 62 & 23 & 205 \\
\hline 1930 & 633 & 444 & 242 & 202 & 114 & 49 & 05 & 25 & 305 \\
\hline 1.931 & 602 & 348 & 202 & 146 & 101 & 48 & 53 & 24 & 223 \\
\hline 1932 & 508 & 331 & 180 & 151 & 84 & 37 & 47 & 30 & 217 \\
\hline 1933 & 519 & 349 & 181 & 168 & 123 & 51 & 72 & 38 & 188 \\
\hline 1934 & 667 & 403 & 224 & 179 & 155 & 70 & 85 & 27 & 221 \\
\hline 1935 & 626 & 377 & 163 & 214 & 127 & 36 & 91 & 34 & 216 \\
\hline 1936 & 669 & 469 & 240 & 229 & 161 & 75 & 86 & 45 & 203 \\
\hline 1937 & 706 & 434 & 212 & 222 & 161 & 77 & 84 & 19 & 254 \\
\hline 1938 & 730 & 486 & 260 & 226 & 139 & 71 & 68 & 35 & 312 \\
\hline 1939 & 734 & 478 & 248 & 230 & 169 & 86 & 83 & 44 & 265 \\
\hline 1940 & $750 \neq$ & $430 \neq$ & 217 & $243 \neq$ & 192 & 97 & 95 & 17 & 251 \\
\hline 19416 & 761 & 482 & 223 & 259 & 229 & 124 & 105 & 21 & 232 \\
\hline
\end{tabular}

* Pacific Coast canned pears are all Bartletts except California canned other varieties as follows in tons: 1935,$400 ; 1930,500 ; 1937,500 ; 1940,10,400$; $1941,10,800$.

+ California dries nearly all the comnercial dried pears in the U. S. These were all Bartletts except 200 tons of other varieties in 1936 and 100 tons in 1940 .

F Excludes 6,550 tons in Washington and 2,000 tons in Oregon of pears harvested but not utilized in accordance with grade and size requirements of marketing agreercents.

$\delta$ Preliminary 1941 official production estimates and unofficial utilization.

Sources of data: Conpiled by S. W. Shear, Giannini foundation of Agricultural Economics, University of California, largely from United States, California, and Oregon Crop Reports, supplemented by carned pack statistics, converted at 38 cases per ton. Data on utilization for 1941 are preliminary trade estimates. 

Table 8. Delivered Auction Prices of Pacific Coast Fresh

Bartletts and California Pears by Chief "arieties, 1922-1941

\begin{tabular}{|c|c|c|c|c|c|c|c|c|}
\hline \multirow{4}{*}{$\begin{array}{l}\text { Crop } \\
\text { year }\end{array}$} & \multicolumn{3}{|c|}{ Bartletts } & \multirow{2}{*}{\multicolumn{5}{|c|}{$\begin{array}{c}\text { Other varieties, California } \\
\text { At New York auction }\end{array}$}} \\
\hline & \multirow{2}{*}{\multicolumn{2}{|c|}{$\begin{array}{l}\text { California, } \\
\text { at all markets }\end{array}$}} & \multirow{3}{*}{$\begin{array}{l}\text { Oregon and } \\
\text { washington, } \\
\text { at New York } \\
\text { auction }\end{array}$} & & & & & \\
\hline & & & & \multirow[b]{2}{*}{ Hardy } & \multirow[b]{2}{*}{ Boso } & \multirow[b]{2}{*}{ Anjou } & \multirow[b]{2}{*}{ Comice } & \multirow[b]{2}{*}{$\begin{array}{l}\text { Winter } \\
\text { Nel is }\end{array}$} \\
\hline & $\begin{array}{l}\text { Calculated } \\
\text { f.o.b. }\end{array}$ & $\begin{array}{c}\text { Delivered } \\
\text { auction }\end{array}$ & & & & & & \\
\hline & 1 & 2 & 3 & 4 & Б & 6 & 7 & 8 \\
\hline & \multicolumn{8}{|c|}{ Dollars per box* } \\
\hline 1922 & 1.49 & 2.75 & 2.21 & 3.03 & 4.09 & 2.85 & 2.96 & 2.98 \\
\hline 1923 & 1.72 & 3.00 & 3.28 & 3.62 & 3.59 & 3.04 & 3.24 & 3.61 \\
\hline 1924 & 2.51 & 3.85 & 3.60 & 4.16 & 4.44 & 3.94 & 4.07 & 4.28 \\
\hline 1925 & 1.49 & 2.75 & 2.99 & 3.43 & 4.15 & 3.38 & 3.38 & 3.57 \\
\hline 1926 & 1.40 & 2.65 & 2.40 & 2.67 & 3.31 & 3.03 & 2.92 & 2.97 \\
\hline 1927 & 2.02 & 3.32 & 3.21 & 3.77 & 4.16 & 4.09 & 4.10 & 3.47 \\
\hline 1928 & 1.66 & 2.86 & 2.75 & 2.74 & 3.16 & 3.01 & 3.15 & 2.80 \\
\hline 1929 & 2.35 & 3.60 & 3.52 & 3.45 & 3.67 & 3.40 & 3.52 & 3.05 \\
\hline 1930 & 1.10 & 2.31 & 1.78 & 2.02 & 2.37 & 2.38 & 2.37 & 2.11 \\
\hline 1931 & 1.36 & 2.60 & 2.67 & 2.83 & 2.65 & 2.87 & 2.94 & 2.20 \\
\hline 1932 & 0.72 & 1.93 & 1.74 & 1.94 & 1.69 & 1.73 & 1.75 & 1.47 \\
\hline 1933 & 1.09 & 2.30 & 1.97 & 1.54 & 1.67 & 2.10 & 1.88 & 2.24 \\
\hline 1934 & 1.40 & 2.52 & 2.45 & 2.25 & 2.08 & 2.50 & 2.42 & 2.12 \\
\hline 1935 & 1.24 & 2.35 & 2.19 & 1.90 & 1.97 & 1.97 & 2.32 & 1.86 \\
\hline 1936 & 1.21 & 2.32 & 2.35 & 2.24 & 2.15 & 2.41 & 2.30 & 2.44 \\
\hline 1937 & 1.34 & 2.45 & 2.27 & 2.10 & 1.97 & 1.89 & 2.62 & 1.67 \\
\hline 1938 & 0.82 & 1.93 & 1.93 & 1.72 & 1.73 & 1.82 & 1.94 & 1.78 \\
\hline 1939 & 1.41 & 2.52 & 2.14 & 1.61 & 1.80 & 1.84 & 1.92 & 1.82 \\
\hline 1940 & 1.21 & 2.32 & 2.16 & 1.77 & 1.71 & 1.84 & 2.17 & 1.91 \\
\hline $1941+$ & 1.68 & 2.79 & 2.77 & 2.88 & 2.81 & 2.34 & 2.58 & 1.65 \\
\hline
\end{tabular}

* Prices are for whole boxes only, except cols. 3-8 in 1922-1925 also include prices of half boxes in equivalent whole boxes.

+ Preliminary through January $30,1942$.

Sources of data:

Compiled by S. W. Shear, Glannini Foundation of Agricultural

Economics, University of California.

Col. 1: Delivered auction prices in col. 2 minus freight and refrigeration charges and a commission of 7 per cent.

Col. 2: Computed directly or indirectly from daily regorts of each auction market; recent years largely from Federal-State larket News Service reports and compilations by other offices of the U.S.D.A.

Cols. 3-8: Based on daily issues of the New York Daily Fruit

Reporter; data for 1926-1936 compiled by U. S. Bur. Agr. Fcon., Division of Statistical and Historical Research. Prices in col. 3 are weighted average prices of Oregon and Washington pears. 

January 1942

\section{PEARS}

Table 9

Fresh Pears: United States Exports and California and Pacific liorthwest Shipments, 1921-1941

\begin{tabular}{|c|c|c|c|c|c|c|c|c|}
\hline \multirow[b]{3}{*}{$\begin{array}{l}\text { Crop } \\
\text { year }\end{array}$} & \multirow{2}{*}{\multicolumn{3}{|c|}{$\begin{array}{l}\text { United States } \\
\text { fresh exports }\end{array}$}} & \multicolumn{4}{|c|}{ Fail shipments } & \\
\hline & & & & \multicolumn{3}{|c|}{$\begin{array}{l}\text { California } \\
\text { interstate }\end{array}$} & \multicolumn{2}{|c|}{$\begin{array}{l}\text { Oregon, Wash. and } \\
\text { Idaho, total* }\end{array}$} \\
\hline & $\begin{array}{l}\text { Total, } \\
\text { years } \\
\text { begin- } \\
\text { ning } \\
\text { July } 1\end{array}$ & $\begin{array}{l}\text { July } \\
\text { and } \\
\text { Aug.t }\end{array}$ & $\begin{array}{l}10 \text { months } \\
\text { beginning } \\
\text { sept. } 1\end{array}$ & $\begin{array}{c}\text { Total, } \\
\text { June } \\
\text { to } \\
\text { Lec. } 31 \\
\end{array}$ & $\begin{array}{l}\text { June, } \\
\text { July, } \\
\text { and, } \\
\text { Aug.t }\end{array}$ & $\begin{array}{c}4 \text { months } \\
\text { beginning } \\
\text { sept. } 1\end{array}$ & $\begin{array}{c}\text { Season's } \\
\text { total }\end{array}$ & $\begin{array}{l}\text { Season } \\
\text { through } \\
\text { Aug. } 31\end{array}$ \\
\hline \multirow{4}{*}{$\begin{array}{l}1921 \\
1922\end{array}$} & 1 & 2 & 3 & 4 & 5 & 6 & 7 & 8 \\
\hline & \multicolumn{3}{|c|}{ Short tons } & \multicolumn{4}{|c|}{ Cars } & \\
\hline & 11,650 & - & - & 4,291 & 3,557 & 734 & 3,900 & 1,792 \\
\hline & 18,392 & 4,745 & 13,647 & 5,751 & 4,240 & 1,511 & 4,560 & 1,341 \\
\hline 1923 & 25,119 & 6,730 & 18,389 & 7,020 & 6,144 & 876 & 6,869 & 2,402 \\
\hline 1924 & 20,726 & 5,663 & 15,063 & 5,804 & 4,948 & 858 & 3,965 & 1,612 \\
\hline 1925 & 35,603 & 8,010 & 27,593 & 7,767 & 6,488 & 1,279 & 5,794 & 1,855 \\
\hline 1926 & 36,939 & 10,022 & 26,917 & 9,826 & 8,602 & 1,224 & 8,208 & 3,522 \\
\hline 1927 & 25,528 & 6,804 & 18,724 & 8,053 & 6,287 & 1,766 & 5,570 & 1,080 \\
\hline 1928 & 41,424 & 11,291 & 30,133 & 9,456 & 7,528 & 1,928 & 10,336 & 3,223 \\
\hline 1929 & 31,012 & 6,786 & 24,226 & 7,624 & 5,302 & 2,322 & 8,275 & 1,434 \\
\hline 1930 & 67,335 & 14,011 & 53,324 & 10,979 & 8,483 & 2,496 & 11,319 & $2,5 j 1$ \\
\hline 1931 & 45,351 & 16,760 & 28,591 & $7,634 \neq$ & 6,787 & 839 & 7,482 & 2,297 \\
\hline 1932 & 59,993 & 13,993 & 46,000 & $5,923 \neq$ & 5,086 & 832 & 7,327 & 1,347 \\
\hline 1933 & 55,503 & 7,593 & 47,910 & 4,497 & 2,941 & 1,556 & 7,187 & 757 \\
\hline 1934 & 50,161 & 13,058 & 34,103 & 5,484 & 4,736 & 748 & 7,200 & 3,094 \\
\hline 1935 & 62,072 & 9,307 & 52,705 & 3,825 & 2,932 & 893 & 9,271 & 1,395 \\
\hline 1936 & 65,645 & 16,528 & 49,117 & 4,939 & 3,972 & 967 & 9,497 & 2,032 \\
\hline 1937 & 67,374 & 13,568 & 53,806 & 5,337 & 3,965 & 1,372 & 9,783 & 932 \\
\hline 1938 & 85,483 & 19,719 & 65,767 & 5,696 & 4,086 & 1,010 & 9,192 & 1,050 \\
\hline 1939 & 46,530 & 14,257 & 32,273 & 4,274 & 3,148 & 1,126 & 8,590 & 1,954 \\
\hline 1940 & 11,783 & 4,382 & 7,401 & 4,431 & 3,147 & 1,284 & 9,012 & 1,922 \\
\hline 1941 & & 3,621 & & 2,993 & 2,350 & 643 & 8,9086 & 1,430 \\
\hline
\end{tabular}

* Includes interstate and intrastate rail shipments.

+ June, July and August exports are tearly all California Bartletts and Hardys, and California interstate shipments in these months are very largely Bartletts.

F Total includes pears ori inating north of Roseville of $8 \mathrm{cars}$ in 1931 and

5 cars in 1932 which are not included in cols. 5 and ö for these years.

fiNor thwest shipment through Feb. 14, 1942.

Sources of data: Compiled by S. T. Shear, Giannini Foundation of Agricultural

Economics, University of California.

Cols. 1-3: From lionthly Summary oi Foreign Commerce.

Cols. 4-8: From reports of Federal-state Narket ews Service. 


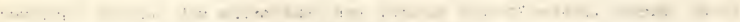
$\therefore \quad: \because \ldots$.

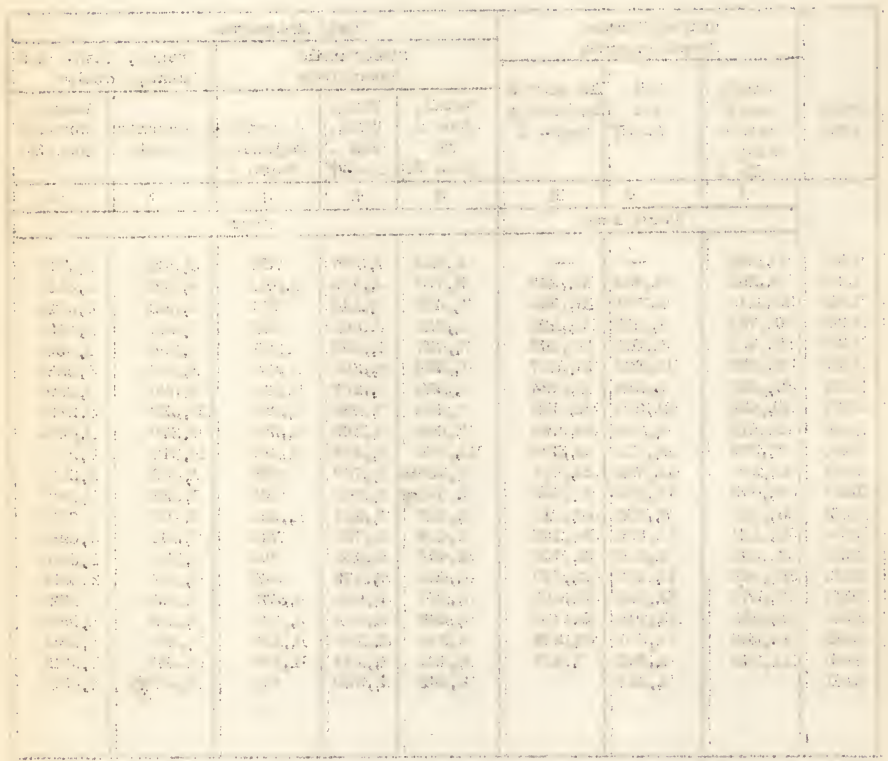

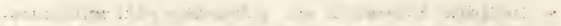

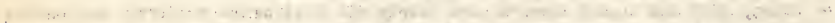

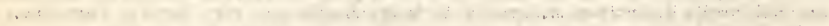

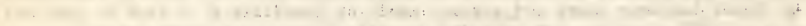

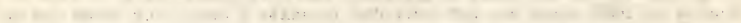
. $10 .+\ldots+\ldots$

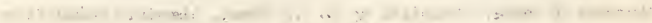

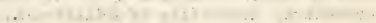

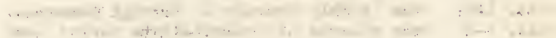


Table 10. Fresh Pears: United States Exports by Countries of Destination, Years Beginning July 1, 1930-1940

\begin{tabular}{|c|c|c|c|c|c|c|c|c|c|c|c|}
\hline $\begin{array}{l}\text { Country of } \\
\text { destination }\end{array}$ & $1930-31$ & $1931-32$ & $1932-33$ & $1933-34$ & $1934-35$ & $1935-36$ & $1936-37$ & $1937-38$ & $1938-39$ & $1939-40 *$ & $1940-41 t$ \\
\hline & \multicolumn{11}{|c|}{ Short tons, net declared fresh weight } \\
\hline Total exports & 67,335 & 45,351 & 59,994 & 55,504 & 50,318 & 62,072 & 65,659 & 67,374 & 85,486 & $4 C, 530$ & $11,498+$ \\
\hline Europe, total & 46,165 & 34,208 & 50,818 & 46,672 & 38,799 & 46,126 & 44,333 & 50,261 & 71,509 & 26,700 & 304 \\
\hline United Kingdom & 34,490 & 23,720 & 30,532 & 27,971 & 26,513 & 29,331 & 26,610 & 29,923 & 47,298 & 22,086 & 129 \\
\hline Other & 11,675 & 10,488 & 20,286 & 18,701 & 12,286 & 16,795 & 17,723 & 20,338 & 24,211 & 4,614 &, 275 \\
\hline France & 866 & 5,006 & 10,460 & 9,916 & 7,420 & 9,684 & 9,797 & 9,312 & 7,614 & 0 & 0 \\
\hline Netherlands & 4,906 & 2,294 & 5,300 & 5,344 & 1,611 & 2,676 & 2,900 & 3,424 & 7,087 & 288 & 0 \\
\hline Belgium & 564 & 360 & 11 & 252 & 32 & 216 & 512 & 1,280 & 1,802 & 61 & 0 \\
\hline Norway & 50 & 145 & 170 & 100 & 220 & 122 & 168 & 205 & 600 & 348 & 0 \\
\hline Sweden & 1,588 & 1,208 & 1,758 & 1,190 & 2,381 & 2,796 & 3,010 & 3,872 & 4,295 & 3,164 & 53 \\
\hline Denmark & 235 & 650 & 185 & 358 & 200 & 0 & $--\neq$ & 35 & 55 & 0 & 0 \\
\hline Germany $\oint$ & 3,310 & 769 & 2,344 & 1,477 & 134 & 762 & 594 & 1,481 & 1,408 & - & 0 \\
\hline Other & 156 & 56 & 58 & 64 & 288 & 539 & 742 & 729 & 1,350 & 753 & 122 \\
\hline Except Europe & 21,170 & 11,143 & 9,176 & 8,832 & 11,519 & 15,946 & 21,326 & 17,113 & 13,977 & 19,830 & 11,194 \\
\hline Canada & 15,051 & 8,137 & 5,908 & 4,518 & 6,187 & 9,652 & 13,984 & 8,943 & 6,755 & 13,174 & 5,621 \\
\hline Other & 6,119 & 3,006 & 3,268 & 4,314 & 5,332 & 6,294 & 7,342 & 8,170 & 7,222 & 6,656 & 5,573 \\
\hline Brazil & 2,376 & 1,036 & 1,404 & 1,735 & 1,818 & 1,981 & 2,614 & 2,298 & 2,344 & 3,192 & 3,046 \\
\hline Argentina & 1,670 & 739 & 716 & 580 & 390 & 356 & 206 & 169 & 254 & 30 & 65 \\
\hline Cuba & 607 & 410 & 329 & 197 & 439 & 524 & 670 & 757 & 711 & 666 & 724 \\
\hline Mexico & 402 & 54 & 80 & 68 & 94 & 96 & 198 & 312 & 279 & 281 & 346 \\
\hline Other & 1,064 & 767 & 739 & 1,734 & 2,591 & 3,337 & 3,654 & 4,634 & 3,634 & 2,487 & 1,392 \\
\hline
\end{tabular}

* Preliminary.

† Nine months only, July, 1940 - March, 1941, as data by countries of destination not available for later months.

Total exports for July - June were 11,783 tons.

$\neq$ Dashes indicate less than 0.5 tons.

f Germany includes Austria beginning kiay 6,1938, and parts of Czechoslovakia and Memel acquired since then.

Sources of data: Compiled by S. W. Shear, Giannini Foundation of Agricultural Economics, University of California, from official data of U. S. Bur. of For. and Dom. Com. largely as compiled by Office of Foreign Agricultural

Relations, U. S. Dept. Agr., and U. S. Dept. Agr., Agricultural Statistics, 1935 and 1939. 


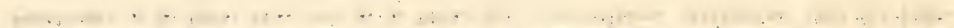

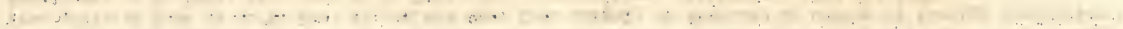

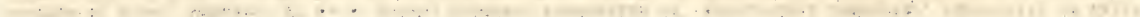

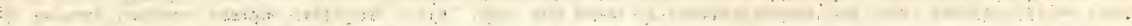

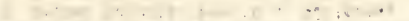
$\therefore \quad \therefore \quad \therefore \quad \div \ldots \div \ldots, \cdots, \cdots$

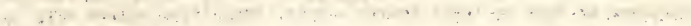
$+\cdots, \cdots: \cdots$

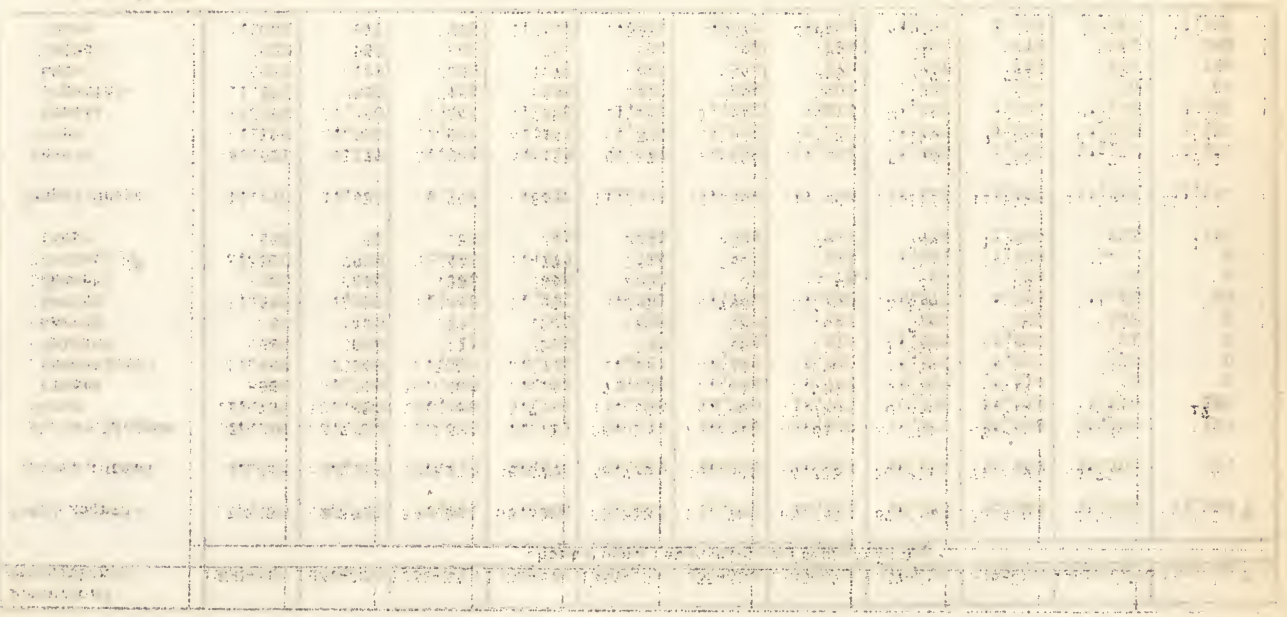

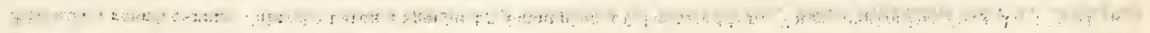


Table 11

Pacific Coast Canned Bartlett Pack, Carryover, Shipments, Exports, and Prices, and California Canning Price, 1922-1941

\begin{tabular}{|c|c|c|c|c|c|c|c|c|c|c|}
\hline \multirow[b]{3}{*}{$\begin{array}{l}\text { Year } \\
\text { begin- } \\
\text { ning } \\
\text { June } 1\end{array}$} & \multicolumn{9}{|c|}{ Pacific Coast } & \multirow{3}{*}{$\begin{array}{l}\text { Calif- } \\
\text { ornia } \\
\text { caming } \\
\text { price } \\
\text { paid } \\
\text { growers } \\
\text { per ton }\end{array}$} \\
\hline & \multirow[b]{2}{*}{ Pack } & \multirow{2}{*}{$\begin{array}{c}\text { carry } \\
\text { over } \\
\text { from } \\
\text { previous } \\
\text { year }\end{array}$} & \multirow{2}{*}{$\begin{array}{l}\text { Avail- } \\
\text { able } \\
\text { for } \\
\text { ship- } \\
\text { ment }\end{array}$} & \multirow{2}{*}{$\begin{array}{l}\text { Carry- } \\
\text { over } \\
\text { into } \\
\text { foll ow- } \\
\text { ing year }\end{array}$} & \multirow{2}{*}{$\begin{array}{l}\text { Total } \\
\text { ship- } \\
\text { ments }\end{array}$} & \multirow{2}{*}{$\begin{array}{c}\text { Domestic } \\
\text { ship- } \\
\text { ments }\end{array}$} & \multicolumn{2}{|c|}{$\begin{array}{c}\text { United States } \\
\text { Exports } *\end{array}$} & \multirow{2}{*}{$\begin{array}{l}\text { F.o.b. } \\
\text { prices } \\
\text { received } \\
\text { by canners } \\
\text { per case }\end{array}$} & \\
\hline & & & & & & & $\begin{array}{l}\text { Quan- } \\
\text { tity }\end{array}$ & $\begin{array}{c}\text { Fer cent } \\
\text { of } \\
\text { shipments }\end{array}$ & & \\
\hline & 1 & 2 & 3 & 4 & 5 & 6 & 7 & 8 & 9 & 10 \\
\hline & \multicolumn{7}{|c|}{ Thous ands of cases, equivalent 24 No. $2 \frac{1}{2}$ cans } & Per cent & \multicolumn{2}{|c|}{ Dollars } \\
\hline 1922 & 2,432 & 50 & 2,482 & 408 & 2,074 & 1,010 & $1,0,4$ & 51 & 5.21 & 70 \\
\hline 1923 & 1,713 & 408 & 2,121 & 142 & 1,979 & 1,085 & 894 & 45 & 4.67 & 35 \\
\hline 1924 & 2,119 & 142 & 2,261 & 51 & 2,210 & 1,014 & 1,196 & 54 & 5.40 & 55 \\
\hline 1925 & 3,429 & 51 & 3,480 & 507 & 2,973 & 1,293 & 1,680 & 57 & 5.44 & 70 \\
\hline 1926 & 3,260 & 507 & 3,767 & 401 & 3,366 & 1,957 & 1,409 & 42 & 4.31 & 37 \\
\hline 1927 & 2,639 & 401 & 3,040 & 167 & 2,873 & 1,637 & 1,230 & 43 & 4.60 & 44 \\
\hline 1928 & 4,116 & 167 & 4,283 & 292 & 3,991 & 2,170 & 1,821 & 46 & 4.13 & 40 \\
\hline 1929 & 4,206 & 292 & 4,498 & 952 & 3,546 & 2,383 & 1,163 & 33 & 4.82 & 80 \\
\hline 1930 & 4,153 & 952 & 5,105 & 893 & 4,212 & 2,617 & 1,595 & 38 & 3.53 & 30 \\
\hline 1931 & 3,635 & 893 & 4,528 & 870 & 3,658 & 1,990 & 1,638 & 46 & 2.82 & 20 \\
\hline 1932 & 3,117 & 870 & 3,987 & 429 & 3,558 & 2,200 & 1,358 & 38 & 2.48 & 14 \\
\hline 1933 & 4,377 & 429 & 4,806 & 273 & 4,533 & 2,767 & 1,760 & 39 & 2.64 & 17 \\
\hline 1934 & 5,505 & 273 & 5,778 & 1,291 & 4,487 & 2,984 & 1,503 & 33 & 3.05 & 35 \\
\hline 1935 & 4,230 & 1,291 & 5,521 & 957 & 4,564 & 2,670 & 1,894 & 41 & 2.92 & 32 \\
\hline 1936 & 5,355 & 957 & 6,312 & 850 & 5,462 & 3,997 & 1,465 & 27 & 2.92 & 26 \\
\hline 1937 & 4,321 & 850 & 5,171 & 1,150 & 4,021 & 2,681 & 1,340 & 33 & 3.07 & 25 \\
\hline 1938 & 4,090 & 1,150 & 5,240 & 400 & 4,840 & 3,114 & 1,720 & 36 & 2.77 & 13 \\
\hline 1939 & 4,057 & 400 & 4,457 & 280 & 4,177 & 2,768 & 1,409 & 34 & 3.27 & 27 \\
\hline 1940 & 4,824 & 280 & 5,104 & 900 & 4,204 & 4,150 & 54 & 1 & $3.06+$ & 28 \\
\hline 1941 & 5,500 & 900 & $6,400+1$ & & & & & & & $42+$ \\
\hline
\end{tabular}

* Exports exolude pears in canned fruit salad and cocktail, but data on pack and shipments include some califomia pears repacked into salad and cocktail.

+ Preliminary: subject to revision.

Sources of data: Compiled by the Giannini Foundation of Agricultural Economics. Cols. 1, 2, and 4: From records of canners cooperation through canners League of California and Northwest Canners Association, exoept Pacific Northwest carryovers included for June 1, 1937-1941 are estimates from incomplete data. cols. 3, 5, 6, and 8: calculated.

Col. 7: U. S. ionthly Sumnary of Foreign Commerce, converted at $45 \mathrm{lbs} \cdot /$ case

Col. 9: jeighted avera,e prices of all grades and sizes of cans on an unadvertised basis and excluding trade discounts, as compiled by $H$. R. Wellman from canners' reports.

Col. 10: Based upon data of the California Pear Growers Association and data secured informally from canners. 


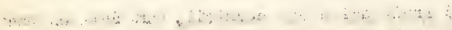

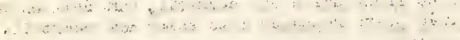

*

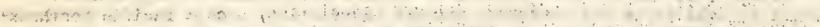

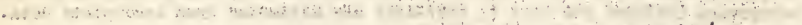

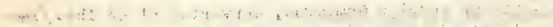

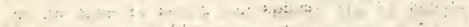


$\mathrm{Table} 12$

Dried Pears: California* Production, Exports, and Packers' F.o.b. Prices, 1921-1941

\begin{tabular}{|c|c|c|c|c|c|c|}
\hline \multirow[b]{3}{*}{ Crop year } & \multirow[b]{3}{*}{$\begin{array}{l}\text { California } \\
\text { production }\end{array}$} & \multicolumn{4}{|c|}{ United states exports } & \multirow{3}{*}{$\begin{array}{l}\text { Packers' } \\
\text { quotations } \\
\text { per pound, } \\
\text { choice } \\
\text { Northerms }\end{array}$} \\
\hline & & \multicolumn{2}{|c|}{ Total } & \multirow{2}{*}{$\begin{array}{c}\text { As } \\
\text { pears }\end{array}$} & \multirow{2}{*}{$\begin{array}{l}\text { As } \\
\text { salad }\end{array}$} & \\
\hline & & $\begin{array}{c}\text { Per cent } \\
\text { of } \\
\text { production }\end{array}$ & Quantity & & & \\
\hline \multirow[t]{2}{*}{$\bar{c}$} & 1 & 2 & 3 & 4 & 5 & 6 \\
\hline & $\begin{array}{l}\text { Short tons, } \\
\text { dry weight }\end{array}$ & Per cent & \multicolumn{3}{|c|}{$\begin{array}{l}\text { Short tons, unprocessed } \\
\text { dry weight }\end{array}$} & Cents \\
\hline 1921 & 1,200 & $--t$ & - & - & -- & 15.4 \\
\hline 1922 & 5,000 & -- & - & - & -- & 13.1 \\
\hline 1923 & 2,000 & 95.0 & 1,900 & 1,100 & 800 & 8.2 \\
\hline 1924 & 3,200 & 40.6 & 1,300 & 1,000 & 300 & 17.3 \\
\hline 1925 & 3,500 & 68.6 & 2,400 & 2,000 & 400 & 14.9 \\
\hline 1926 & 4,300 & 62.8 & 2,700 & 2,200 & 500 & 9.1 \\
\hline 1927 & 3,500 & 71.4 & 2,500 & 1,900 & 600 & 10.4 \\
\hline 1928 & 5,600 & 80.4 & 4,500 & 3,700 & 800 & 10.0 \\
\hline 1929 & 4,200 & 69.0 & 2,900 & 1,800 & 1,100 & 15.1 \\
\hline 1930 & 4,500 & $108.9 \neq$ & 4,900 & 3,700 & 1,200 & 7.1 \\
\hline 1931 & 4,400 & 93.2 & 4,100 & 2,900 & 1,200 & 6.6 \\
\hline 1932 & 5,500 & 76.4 & 4,200 & 3,100 & 1,100 & 5.7 \\
\hline 1933 & 7,000 & 70.0 & 4,900 & 4,000 & 900 & 6.8 \\
\hline 1934 & 4,900 & 75.5 & 3,700 & 2,800 & 900 & 7.6 \\
\hline 1935 & 6,100 & 77.0 & 4,700 & 3,600 & 1,100 & 7.7 \\
\hline 1936 & 8,100 & 51.9 & 4,200 & 3,500 & 700 & 7.3 \\
\hline 1937 & 3,500 & $114.3 \neq$ & 4,000 & 2,900 & 1,100 & 5.9 \\
\hline 1938 & 6,500 & 86.2 & 5,600 & 4,000 & 1,600 & 6.4 \\
\hline 1939 & 8,100 & 44.4 & 3,600 & 2,400 & 1,200 & 7.3 \\
\hline 1940 & 3,100 & 22.6 & 700 & 400 & 300 & 6.0 \\
\hline 1941 & 3,9009 & & & & & 8.1 \\
\hline
\end{tabular}

States.

* California produces practically all the pears dried in the United

+Dashes indicate data not available.

\# Excess of exports over production probably due to carryin.

\$Preliminary estimate.

Sources of data:

Compiled by S. W. Shear, Giannini Foundation.

Col. 1: 1921-1928 based largely upon packers' receipts as reported

by the Dried Fruit Association of California. 1929-1940 from California

Crop Reports of June. Converted from fresh to dry weight at 5.5 to 1 .

Cols. 3-5: Exports of dried pears and one sixth of exports of dried

fruit salad, years beginning September 1. 1923-1929 estimates by S. T.

Shear based largely on inspections by the Dried Fruit Association of California of dried pears and of dried fruit salad for foreign export. 1930-

1940 from U. S. Bur. Far and Dom. Com., lonthly Summary of Foreign Commerce.

Col. 6: Average of weekly quotations in Cal ifornia Fruit News far seasons through December. 
$\therefore+\cdots$

\author{
$\vdots \ldots+\cdots$

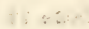

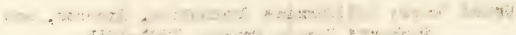

:

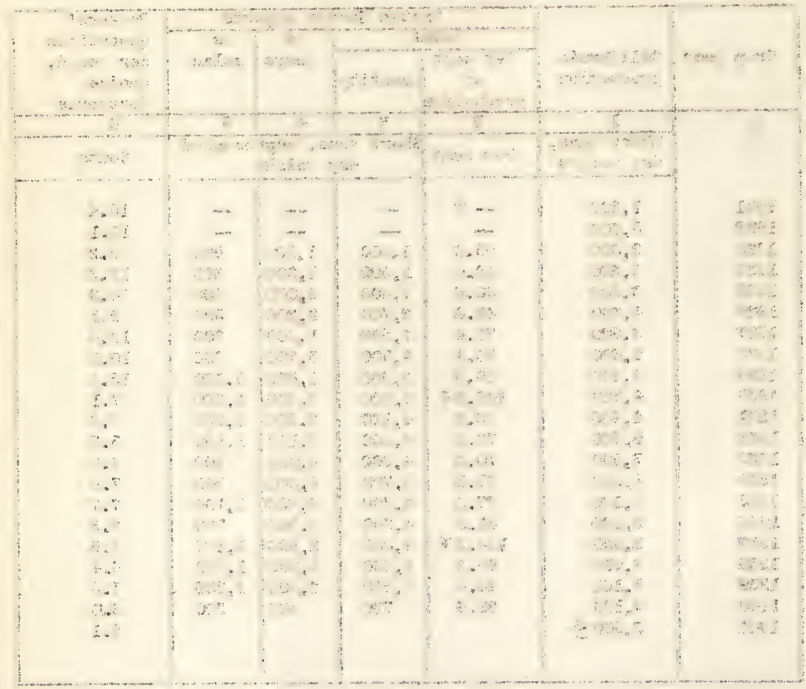

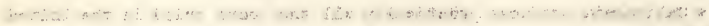

$25:-10$

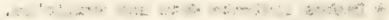

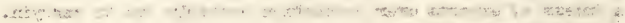

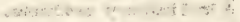

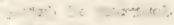

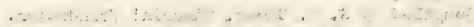

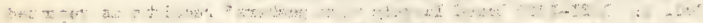

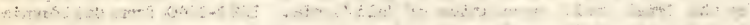

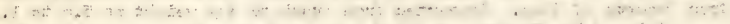

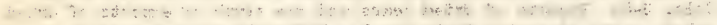

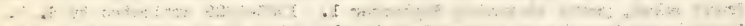

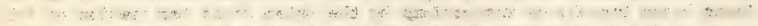

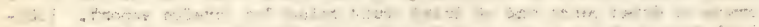

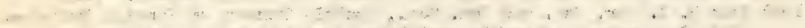

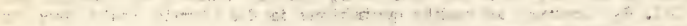

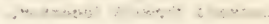


Table 13. Dried Pears*: United States Exports by Countries of Destination, Years Beginning July 1 , 1930-1940

\begin{tabular}{|c|c|c|c|c|c|c|c|c|c|c|c|}
\hline $\begin{array}{l}\text { Country of } \\
\text { destination }\end{array}$ & $1930-31$ & $1931-32$ & $1932-33$ & $1933-34$ & $1934-35$ & $1935-36$ & $1936-37$ & $1937-38$ & $1938-39$ & $1939-40^{+1}$ & $1940-41 \neq$ \\
\hline & \multicolumn{11}{|c|}{ Short tons, net declared dry export weight } \\
\hline Total exports & 4,018 & 3,039 & 3,129 & 4,204 & 2,843 & 3,780 & 3,675 & 2,789 & 4,358 & 2,466 & $306 \neq$ \\
\hline Europe, total & 3,862 & 2,906 & 3,032 & 4,032 & 2,596 & 3,512 & 3,315 & 2,468 & 4,107 & 2,210 & 123 \\
\hline United Kingdom & 587 & 528 & 288 & 269 & 278 & 650 & 439 & 515 & 599 & 607 & 0 \\
\hline Other & 3,275 & 2,378 & 2,744 & 3,763 & 2,318 & 2,862 & 2,876 & 1,953 & 3,508 & 1,603 & 123 \\
\hline France & 712 & 707 & 700 & 626 & 799 & 1,628 & 1,519 & 1,045 & 1,051 & 111 & 0 \\
\hline Netherlsnds & 474 & 212 & 323 & 255 & 278 & 487 & 584 & 233 & 950 & 855 & 0 \\
\hline Belgium & 61 & 31 & 20 & 40 & 53 & 41 & 69 & 47 & 601 & 59 & 0 \\
\hline Norway & 9 & 6 & 2 & 3 & 5 & 6 & 9 & 10 & 25 & 6 & 0 \\
\hline Sweden & 117 & 138 & 168 & 165 & 197 & 300 & 444 & 297 & 401 & 346 & 122 \\
\hline Denmark & 47 & 69 & 16 & 24 & 13 & 2 & 7 & 6 & 126 & 35 & 0 \\
\hline Germany $\mathfrak{s}$ & 1,726 & 1,076 & 1,434 & 2,509 & 824 & 255 & 100 & 110 & 88 & 1 & 0 \\
\hline Other & 129 & 139 & 81 & 141 & 149 & 143 & 144 & 205 & 266 & 190 & 1 \\
\hline Except Europe & 156 & 133 & 97 & 172 & 247 & 268 & 360 & 321 & 251 & 256 & 183 \\
\hline Canada & 57 & 83 & 48 & 90 & 64 & 144 & 209 & 204 & 118 & 148 & 103 \\
\hline Other & 99 & 50 & 49 & 82 & 183 & 124 & 151 & 117 & 133 & 108 & 80 \\
\hline Mexico & 28 & 2 & 7 & 8 & 12 & 12 & 14 & 15 & 14 & 15 & 16 \\
\hline Brazil & 3 & 1 & 3 & 7 & 4 & 4 & 4 & 5 & 6 & 6 & 3 \\
\hline China & 15 & 10 & 8 & 8 & 17 & 9 & 15 & 5 & 3 & 2 & 3 \\
\hline falestine & 3 & 2 & 4 & 25 & 79 & 51 & 36 & 16 & 24 & 7 & 0 \\
\hline Other & 50 & 35 & 27 & 34 & 71 & 48 & 82 & 76 & 86 & 78 & 58 \\
\hline
\end{tabular}

- Do not include exports of dried pears in dried fruit compote or salad (see table 12).

tPreliminary.

† Nine months only, July, 1940 - March, 1941, as data by countries of destination not available for later months. Total exports for July - June were 393 tons.

$\oint$ Germany includes Austria beginning liay 6, 1938, and parts of Czechoslovakia and iemel acquired since then. Source of data: Compiled by S. W. Shear, Giannini Foundation of Agricultural Economics, University of California, from official data of U. S. Bur. For. and Dom. Com., as compiled largely by office of Foreign Agricultural Relations, U. S. Dept. of Agriculture. 
$\therefore \quad \cdots+\cdots$

$\left[\begin{array}{c}0 \\ 2\end{array}\right.$

(2)

-

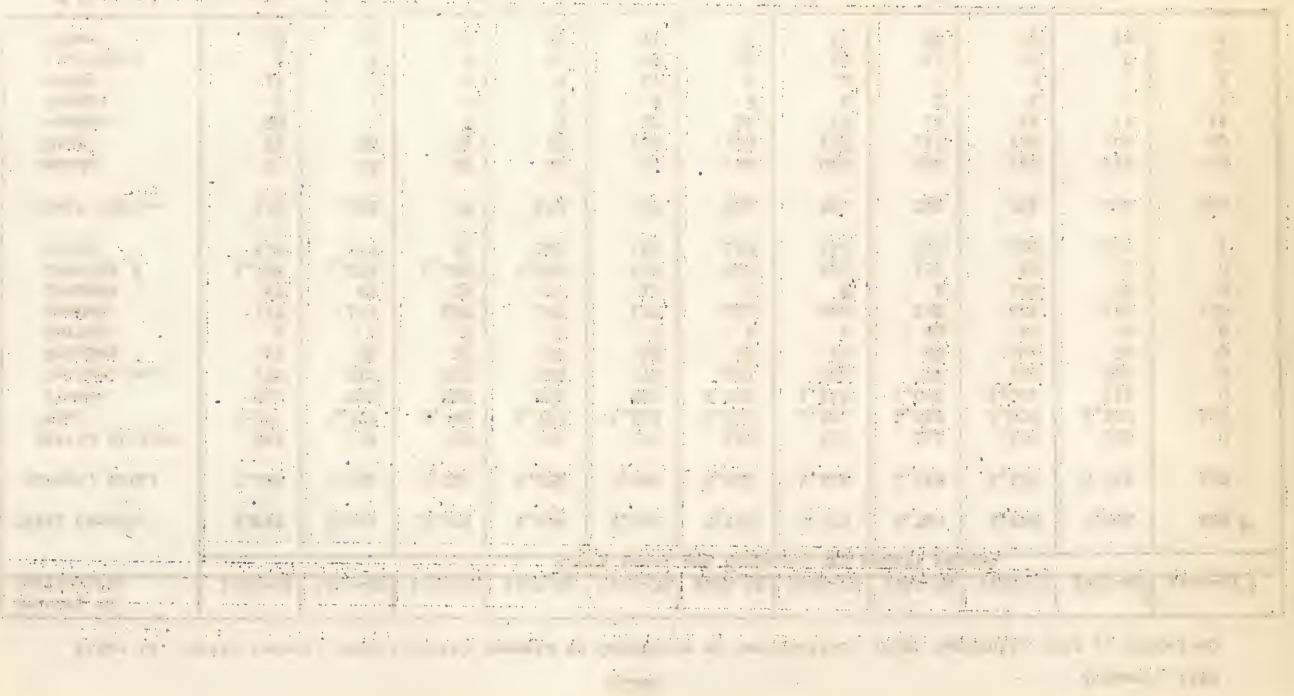


University of California, College of + gricul ture

A Gricultural Experiment Station, Berleley, January 1942

DECIDUOUS FRUIT STITISTICS

PLULIS

Table 1

California Plums: Bearing hereage, Production, Condition, Yield and Farm Value, 1919-1941

\begin{tabular}{|c|c|c|c|c|c|c|}
\hline \multirow[b]{2}{*}{$\begin{array}{l}\text { Crop } \\
\text { year }\end{array}$} & \multirow[b]{2}{*}{$\begin{array}{l}\text { Bearing } \\
\text { acreage }\end{array}$} & \multicolumn{2}{|c|}{ Production } & \multirow[b]{2}{*}{$\begin{array}{l}\text { Yield por } \\
\text { bearing } \\
\text { acre }\end{array}$} & \multicolumn{2}{|c|}{ Farm * value of crop } \\
\hline & & Total & $\begin{array}{l}\text { Sept. I } \\
\text { per cent } \\
\text { of } \\
\text { full crop }\end{array}$ & & $\begin{array}{l}\text { Price to } \\
\text { growers } \\
\text { per ton }\end{array}$ & $\begin{array}{l}\text { Total } \\
\text { returns } \\
\text { to growers }\end{array}$ \\
\hline & $I$ & 2 & 3 & 4 & 5 & 6 \\
\hline & Acres & Short tons & Per cent & Short tons & Dollars & 1,000 dollars \\
\hline 1919 & 17,250 & 42,000 & 90 & 2.4 & 74 & 3,108 \\
\hline 1920 & 17,300 & 35,000 & 75 & 2.0 & 102 & 3,570 \\
\hline 1921 & 19,715 & 42,000 & 69 & 2.1 & 58 & 2,436 \\
\hline 1922 & 22,434 & 48,000 & 82 & 2.1 & 45 & 2,160 \\
\hline 1923 & 23,800 & 69,000 & 100 & 2.9 & 34 & 2,346 \\
\hline 1924 & 25,398 & 39,000 & 60 & 1.5 & 57 & 2,223 \\
\hline 1925 & 28,268 & 51,000 & 65 & 1.8 & 47 & 2,397 \\
\hline 1926 & 30,081 & 71,000 & 87 & 2.4 & 29 & 2,059 \\
\hline 1927 & 33,004 & 57,000 & 64 & 1.7 & 53 & 3,021 \\
\hline 1928 & 33,578 & 66,000 & 80 & 2.0 & 45 & 2,970 \\
\hline 1929 & 32,584 & 40,000 & 45 & 1.2 & 91 & 3,640 \\
\hline 1930 & 31,882 & 82,000 & 87 & 2.6 & 35 & 2,870 \\
\hline 1931 & 31,572 & $65,000 t$ & 74 & 2.1 & 26 & 1,508 \\
\hline 1932 & 32,119 & $68,000 t$ & 77 & 2.1 & 18 & 1,044 \\
\hline 1933 & 31,172 & $57,000+$ & 68 & 1.8 & 26 & 1,300 \\
\hline 1934 & 31,358 & 62,000 & 73 & 2.0 & 32 & 1,984 \\
\hline 1935 & 26,900 & 48,000 & 53 & 1.8 & 39 & 1,872 \\
\hline 1936 & $24,943^{\ddagger}$ & 64,000 & 73 & 2.6 & 30 & 1,920 \\
\hline 1937 & $25,720 \neq$ & 66,000 & 64 & 2.6 & 49 & 3,234 \\
\hline 1938 & $26,032 \neq$ & 63,000 & 68 & 2.4 & 28 & 1,764 \\
\hline 1939 & $25,136 \neq$ & $71,000+$ & 70 & 2.8 & 31 & 1,984 \\
\hline 1940 & $21,960 \neq$ & $69,000 t$ & 74 & 3.1 & 41 & 2,624 \\
\hline 19418 & 22,000 & $74,000 t$ & 72 & 3.4 & 50 & 3,450 \\
\hline & & & & & & \\
\hline
\end{tabular}

* Growers' returns for naked fruit delivered at growers' first delivery point.

t Includes unharvested tonnage: $1931,7,000 ; 1932,10,000 ; 1933,7,000$;

$1939,7,000$; $1940,5,000$; and 1941, 5,000.

₹ Nonbearing acres: 1936, 3,023; 1937, 2,332; 1938, 1,997; 1939, 1,713; and 1940, 2,370.

¿ Preliminary estimates.

Source of data:

Compiled by S. W. Shear, Giannini Foundation of Agrioultural Economics, University of California, largely from reports of the California Cooperative Crop Reporting Service, except cols. 4 and 6 calculated. 
Table 2. California Plums Used Fresh and New York Auction Prices of Important Varieties, 1922-1941

\begin{tabular}{|c|c|c|c|c|c|c|c|c|c|c|}
\hline \multirow[b]{2}{*}{$\begin{array}{l}\text { Crop } \\
\text { year }\end{array}$} & \multicolumn{3}{|c|}{ Used fresh } & \multicolumn{6}{|c|}{ New York auction price per crate } & \multirow[b]{2}{*}{$\begin{array}{c}\text { Calculated } \mathbf{f} .0 . b . \\
\text { price of eleven } \\
\text { varieties }\end{array}$} \\
\hline & Total & $\begin{array}{l}\text { Within- } \\
\text { state }\end{array}$ & $\begin{array}{l}\text { Out of } \\
\text { state }\end{array}$ & Santa Rosa & President & Beauty & Wickson & Tragedy & $\begin{array}{l}\text { Average } \\
\text { of eleven } \\
\text { varieties }\end{array}$ & \\
\hline & 1 & 2 & 3 & 4 & 5 & 6 & 7 & 8 & 9 & 10 \\
\hline & \multicolumn{3}{|c|}{ Tons } & \multicolumn{6}{|c|}{ Dollars } & \\
\hline 1922 & 45,300 & 3,300 & 42,000 & 1.55 & 2.19 & 1.69 & 1.51 & 1.75 & 1.65 & 0.98 \\
\hline 1923 & 66,600 & 3,600 & 63,000 & 1.39 & 2.07 & 1.76 & 1.52 & 1.32 & 1.40 & 0.75 \\
\hline 1924 & 37,700 & 3,100 & 34,600 & 2.06 & 2.63 & 2.21 & 2.23 & 2.14 & 2.06 & 1.36 \\
\hline 1925 & 48,300 & 4,000 & 44,300 & 1.60 & 1.79 & 1.75 & 2.15 & 1.69 & 1.79 & . 1.11 \\
\hline 1926 & 67,600 & 5,000 & 62,600 & 1.30 & 1.79 & 1.60 & 1.42 & 1.50 & 1.45 & 0.79 \\
\hline 1927 & 54,900 & 5,900 & 49,000 & 1.60 & 2.04 & 1.81 & 2.30 & 1.79 & 1.81 & 2.13 \\
\hline 1928 & 63,100 & 7,000 & 56,100 & 1.53 & 2.34 & 2.02 & 1.74 & 1.65 & 1.68 & 1.04 \\
\hline 1929 & 38,000 & 5,500 & 32,500 & 2.32 & 2.18 & 2.23 & 2.91 & 2.86 & 2.36 & 1.67 \\
\hline 1930 & 78,900 & 8,200 & 70,700 & 1.67 & 1.49 & 2.13 & 1.71 & 1.30 & 1.51 & 0.88 \\
\hline 1931 & 56,500 & 8,900 & 47,600 & 1.17 & 1.75 & 1.49 & 1.67 & 1.50 & 1.40 & 0.75 \\
\hline 1932 & 56,800 & 10,100 & 46,700 & 1.17 & 1.60 & 1.15 & 1.33 & 1.39 & 1.25 & 0.61 \\
\hline 1933 & 48,200 & 7,800 & 40,400 & 1.36 & 1.54 & 1.57 & 1.22 & 1.16 & 1.34 & 0.70 \\
\hline 1934 & 60,200 & 8,900 & 51,300 & 1.36 & 1.55 & 1.42 & 1.41 & 1.38 & 1.38 & 0.80 \\
\hline 1935 & 45,900 & 8,900 & 37,000 & 2.04 & 1.90 & 1.60 & 1.60 & 1.43 & 1.60 & 1.00 \\
\hline 1936 & 62,700 & 10,600 & 52,100 & 1.29 & 1.50 & 1.28 & 1.35 & 1.50 & 1.34 & 0.76 \\
\hline 1937 & 63,000 & 12,500 & 50,500 & 1.92 & 1.72 & 1.73 & 1.66 & 2.07 & 1.74 & 1.13 \\
\hline 1938 & 62,000 & 11,600 & 50,400 & 1.66 & 1.27 & 1.34 & 1.23 & 1.27 & 1.35 & 0.74 \\
\hline 1939 & 62,600 & 14,300 & 48,300 & 1.64 & 1.86 & 1.41 & 1.28 & 1.59 & 1.54 & 0.92 \\
\hline 1940 & 63,100 & 10,900 & 52,200 & 1.71 & 1.66 & 2.18 & 1.32 & 1.59 & 1.66 & 1.03 \\
\hline $1941 *$ & 67,300 & 13,600 & 53,700 & 1.86 & 1.73 & 1.73 & 1.66 & 1.85 & 1.76 & 1.12 \\
\hline
\end{tabular}

* Preliminary.

Sources of data: Compiled by S. Whear, Giannini Foundation of Agricultural Economics, University of Cslifornia. Cols. 1-3: 1922-1927 and 1941: Col. 1: Harvested production minus canned tonnage based upon reports of the

Canners League of California; and col. 3: Largély based on Interstate shipments of California Deciduous-Tree

Fruits, Federal-State Market News Service, San Francisco (Mimeo.). 1928-1940: From reports of the California Cooperative Crop Reporting Service.

Cols. 4-9: Weighted average of delivered auction sales compiled largely from the New York Daily Fruit Reporter. Col. 10: Col. 9 minus freight, refrigeration, and 7 per cent sales commission. 


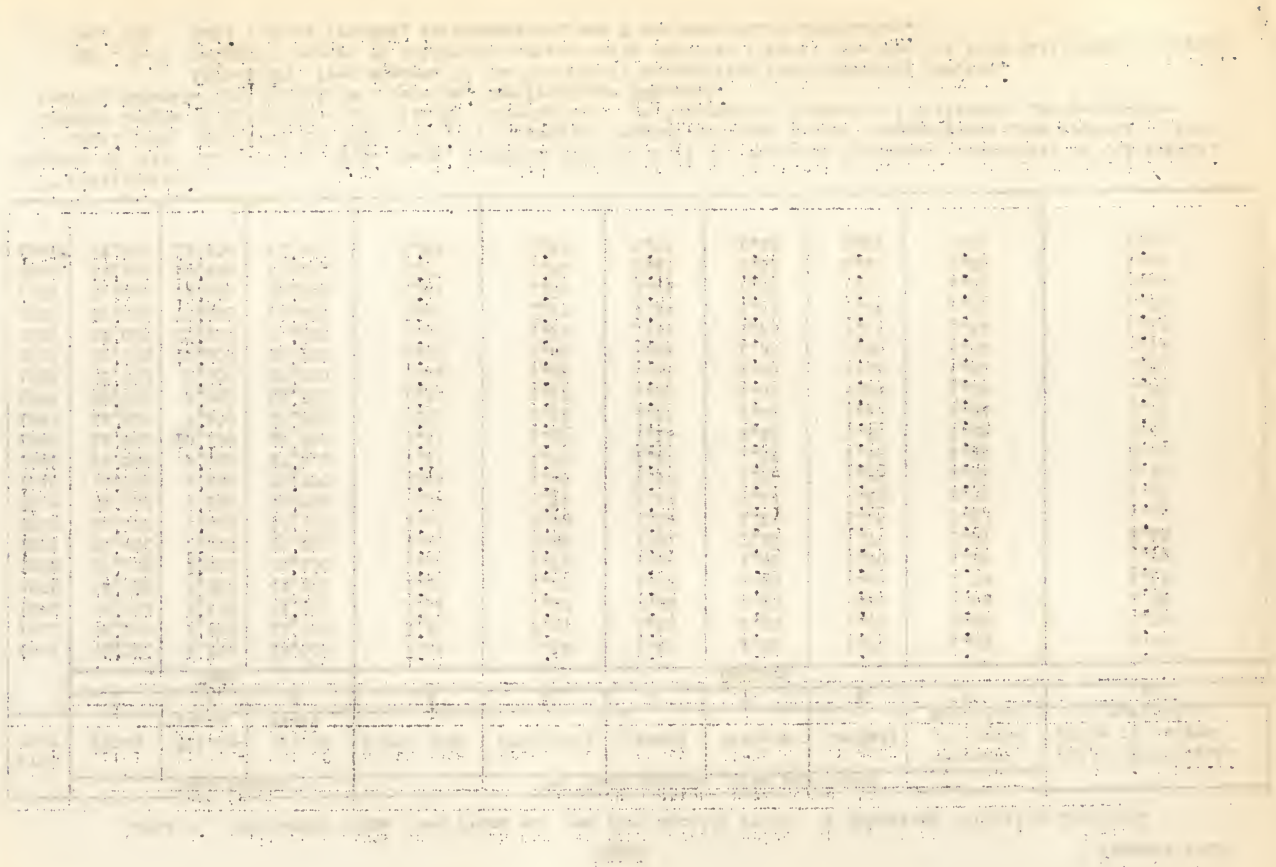




\section{PLUMS}

Table 3

Harvested Production and Canned Pacl: of California and Paciric llor thwest Plums and Fresh Prunes, 1919-1941

\begin{tabular}{|c|c|c|c|c|c|c|}
\hline \multirow{3}{*}{$\begin{array}{l}\text { Crop } \\
\text { year }\end{array}$} & \multicolumn{3}{|c|}{ California } & \multicolumn{3}{|c|}{ Oregon, Washincton, and Idaho * } \\
\hline & \multirow[b]{2}{*}{$\begin{array}{l}\text { Harvested } \\
\text { production }\end{array}$} & \multicolumn{2}{|l|}{ Canned * } & \multirow[b]{2}{*}{$\begin{array}{l}\text { Harvested } \\
\text { production }\end{array}$} & \multicolumn{2}{|c|}{ Canned $* t$} \\
\hline & & $\begin{array}{l}\text { Per cont or } \\
\text { production }\end{array}$ & Quantity & & $\begin{array}{l}\text { Per cent of } \\
\text { production }\end{array}$ & Quantity \\
\hline \multirow{4}{*}{$\begin{array}{l}1919 \\
1920\end{array}$} & 1 & 2 & 3 & 4 & 5 & 6 \\
\hline & Short tons & Per cent & Short tons & Short tons & Per cent & Short tons \\
\hline & 42,000 & 9.5 & 4,000 & 29,400 & 7.8 & 2,300 \\
\hline & 35,000 & 6.9 & 2,400 & 27,200 & 6.2 & 1,700 \\
\hline 1921 & 42,000 & 5.0 & 2,100 & 42,900 & 4.0 & 1,700 \\
\hline 1922 & 48,000 & 5.6 & 2,700 & 31,800 & 13.2 & 4,200 \\
\hline 1923 & 69,000 & 3.5 & 2,400 & 58,100 & 7.1 & 4,100 \\
\hline 1924 & 39,000 & 3.3 & 1,300 & 28,000 & 7.5 & 2,100 \\
\hline 1925 & 51,000 & 5.3 & 2,700 & 33,500 & 13.4 & 4,500 \\
\hline 1926 & 71,000 & 4.8 & 3,400 & 51,900 & 14.5 & 7,500 \\
\hline 1927 & 57,000 & 3.7 & 2,100 & 52,300 & 13.2 & 6,900 \\
\hline 1928 & 66,000 & 4.4 & 2,900 & 66,200 & 15.1 & 10,000 \\
\hline 1929 & 40,000 & 5.0 & 2,000 & 86,000 & 18.7 & 16,100 \\
\hline 1930 & 82,000 & 3.8 & 3,100 & 70,700 & 13.8 & 13,300 \\
\hline 1931 & 58,000 & 2.6 & 1,500 & 57,200 & 24.0 & 13,700 \\
\hline 1932 & 58,000 & 2.1 & 1,200 & 57,700 & 17.7 & 10,200 \\
\hline 1933 & 50,000 & 3.6 & 1,800 & 52,500 & 28.4 & 14,900 \\
\hline 1934 & 62,000 & 2.9 & 1,800 & 67,900 & 27.7 & 18,800 \\
\hline 1935 & 48,000 & 4.1 & 2,100 & 77,300 & 36.2 & 28,000 \\
\hline 1936 & 64,000 & 2.0 & 1,300 & 77,200 & 40.2 & 31,000 \\
\hline 1937 & 66,000 & 4.5 & 3,000 & 65,400 & 43.7 & 28,600 \\
\hline 1938 & 63,000 & 1.6 & 1,000 & 63,800 & 24.0 & 15,300 \\
\hline 1939 & 64,000 & 2.2 & 1,400 & 90,700 & 35.8 & 32,500 \\
\hline 1940 & 64,000 & 1.4 & 900 & 66,810 & 29.9 & 20,000 \\
\hline $1941 \neq$ & 69,000 & 2.5 & 1,700 & 85,800 & 43.9 & 37,700 \\
\hline & & & & & & \\
\hline
\end{tabular}

* The few hundred tons canned in Idaho omitted from the canned tonnage.

$t$ Includes small quantities for cold packing.

F Preliminary estimates.

Sources of data:

Compiled by S. T. Shear, Giannini Foundation of Agricultural Economics, University of California.

Col. 1: 1919-1936 from U. S. Dept. Lgr. Bur. Agr. Lcon., Revised Production of Plums and Prunes, 1919-1936 mimeo. July 30, 1937. 1937-1941

from U. S. Crop Reports.

Col. 3: 1919-1927 and 1941 based on reports of Canners League of California; equivalent cases of $24 \mathrm{Ho}$. $2 \frac{1}{\mathrm{~s}}$ cans converted at 63 cases per ton, except 1941 at 62. 1928-1940 from repor ts of Californie. Cooperative Crop Reporting Service.

Cols. 4 and 6: From U. S. Dept. Agr. General Crop keport. Dec. 1939, 1940 , and 1941 . 



\section{Table 4}

United States Harvested Production* of Plums and Fresh Prunes,

\begin{tabular}{|c|c|c|c|c|c|c|c|c|}
\hline \multirow[b]{2}{*}{$\begin{array}{l}\text { Crop } \\
\text { year }\end{array}$} & \multirow[b]{2}{*}{$\begin{array}{l}\text { Grand } \\
\text { total }\end{array}$} & \multirow[b]{2}{*}{$\begin{array}{c}\text { Pacific } \\
\text { Coast }\end{array}$} & \multirow[b]{2}{*}{$\begin{array}{l}\text { Cali- } \\
\text { fornia }\end{array}$} & \multicolumn{4}{|c|}{ Pacific Nor thwest } & \multirow[b]{2}{*}{ lichigan } \\
\hline & & & & Total & $\begin{array}{l}\text { Washing- } \\
\text { ton }\end{array}$ & Oregon & Idaho & \\
\hline \multirow[b]{3}{*}{1919} & 1 & 2 & 3 & 4 & 5 & 6 & 7 & 8 \\
\hline & \multicolumn{8}{|c|}{ Short tons, fresh weight } \\
\hline & 77,100 & 71,400 & 42,000 & 29,400 & 11,100 & 4,700 & 13,600 & 5,700 \\
\hline 1920 & 69,500 & 62,200 & 35,000 & 27,200 & 6,000 & 5,300 & 15,900 & 7,300 \\
\hline 1921 & 87,600 & 84,900 & 42,000 & 42,900 & 8,100 & 11,500 & 23,300 & 2,700 \\
\hline 1922 & 87,900 & 79,800 & 48,000 & 31,800 & 4,300 & 12,500 & 15,000 & 8,100 \\
\hline 1923 & 131,600 & 127,100 & 69,000 & 58,100 & 9,100 & 22,000 & 27,000 & 4,500 \\
\hline 1924 & $72,800 *$ & $67,000 *$ & 39,000 & $28,000 *$ & 8,100 & 9,900 & $10,000 *$ & 5,800 \\
\hline 1925 & 88,200 & 84,500 & 51,000 & 33,500 & 9,700 & 11,800 & 12,000 & 3,700 \\
\hline 1926 & $131,300 *$ & $122,900 *$ & 71,000 & $51,900 *$ & 12,900 & 20,500 & $18,500 *$ & 8,400 \\
\hline 1927 & 113,200 & 109,300 & 57,000 & 52,300 & 12,300 & 18,500 & 21,500 & 3,900 \\
\hline 1928 & 139,100 & 132,200 & 66,000 & 66,200 & 18,500 & 25,500 & 22,200 & 6,900 \\
\hline 1929 & $130,400 *$ & $126,000 *$ & 40,000 & $86,000 *$ & 24,400 & $34,200 *$ & 27,400 & 4,400 \\
\hline 1930 & $160,100 *$ & $152,700 *$ & 82,000 & $70,700 *$ & $21,400 *$ & $27,000 *$ & $22,300 *$ & 7,400 \\
\hline 1931 & $122,200 *$ & $115,200 *$ & $58,000 *$ & $57,200 *$ & 12,000 & $24,100 *$ & 21,100 & 7,000 \\
\hline 1932 & $121,900 *$ & $115,700 *$ & $58,000 *$ & $57,700 *$ & $14,200 *$ & $21,500 *$ & $22,000 *$ & 6,200 \\
\hline 1933 & $106,900 *$ & $102,500 *$ & $50,000 *$ & $52,500 *$ & $19,200 *$ & $27,000 *$ & 6,300 & 4,400 \\
\hline 1934 & $135,400 *$ & $129,900 *$ & 62,000 & $67,900 *$ & $21,100 *$ & $34,300 *$ & 12,500 & 5,500 \\
\hline 1935 & $131,300 *$ & $125,300 *$ & 48,000 & $77,300 *$ & $21,400 *$ & $34,600 *$ & 21,300 & 6,000 \\
\hline 1936 & 145,500 & 141,200 & 64,000 & 77,200 & 19,900 & 44,200 & 13,100 & 4,300 \\
\hline 1937 & $137,200 *$ & $131,400 *$ & 66,000 & $65,400 *$ & $15,500 *$ & $37,000 *$ & 12,900 & 5,800 \\
\hline 1938 & $129,700 *$ & $126,800 *$ & 63,000 & $63,800 *$ & $18,400 *$ & $30,200 *$ & $15,200 *$ & 2,900 \\
\hline 1939 & $161,000 *$ & $154,700 *$ & $64,000 *$ & $90,700 *$ & $22,600 *$ & $45,800 *$ & $22,300 *$ & 6,300 \\
\hline 1940 & $136,610 *$ & $130,810 *$ & $64,000 *$ & $66,810 *$ & 17,110 & $28,200 *$ & 21,500 & 5,800 \\
\hline 1941月 & $161,600 *$ & $154,800 *$ & $69,000 *$ & 85,800 & 18,300 & 45,400 & 22,100 & 6,800 \\
\hline
\end{tabular}

* Excludes tomnace unharvested on account of marlset conditions. Idaho: 1924, 2,$000 ; 1926,1,000 ; 1930,1,000 ; 1952,4,000 ; 1938,500$; and 1939, 1,200 : California: $1931,7,000 ; 1932,10,000 ; 1933,7,000 ; 1939,7,000 ; 1940,5,000$; and 1941 , 5,000. Also, unharvested production in Oregon and Washincton which is reported with the dried prunes is excluded from above table.

+ California and Mlichican data are mostly plums and prunes used fresh; Idaho, mostly prunes used fresh; Washington and Oregon, prunes used fresh and for conning. $\neq$ Preliminary estimates.

Sources of data: Compiled by S. W. Shear, Giannini Founclation of figrioultural

Economics, University of California.

Cols. 1, 2, and 4: Calculated from data in cols. 3 and 5-8.

Cols. 3, 7, and 8: 1019-1936 from U. S. Dept. Agr. Bur. Agr. Econ.,

Revised Production of Pluns and Prunes, 1919-1936 mimeo. July 30, 1937. 1937-

1941 from United Statos Crop Reports.

Cols. 5 and 6: From U. S. Dept. Agr. General Crop Report. December

1939,1940 , and 1941 . 


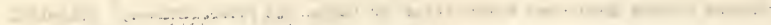

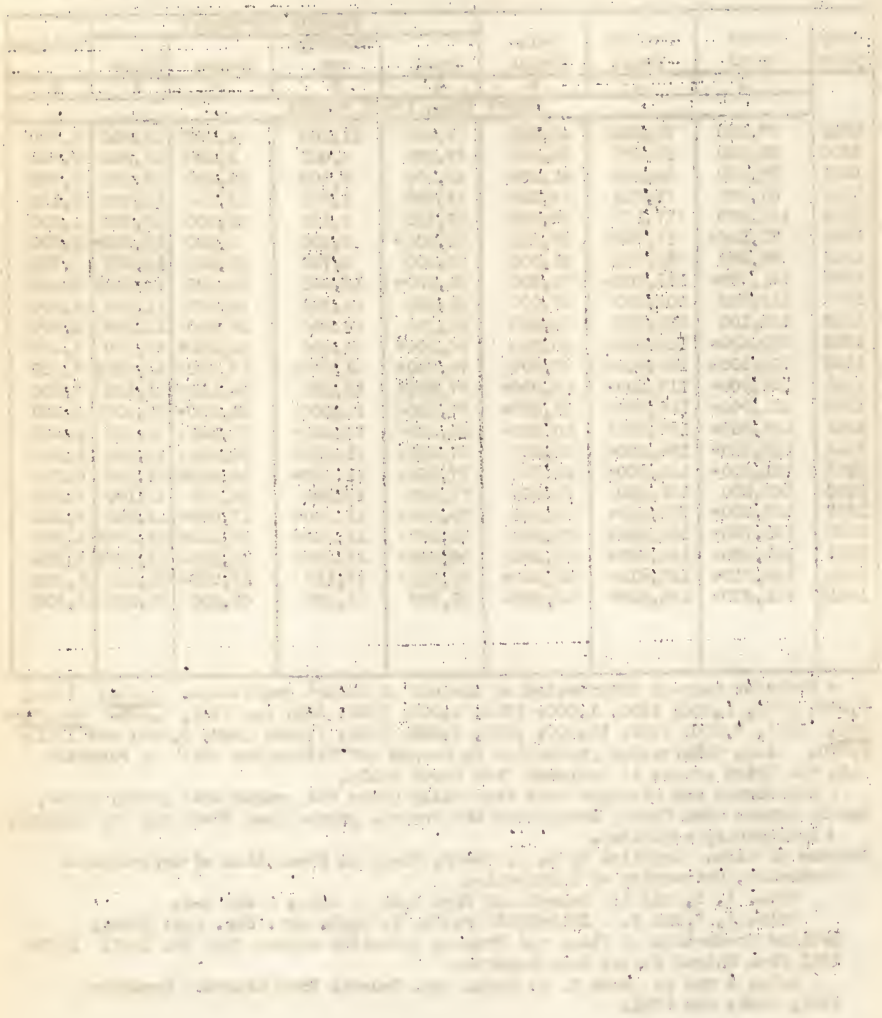


University of California, College of Agriculture

Agricultural Experiment Station, Berkeley, January, 1942

DECIDUOUS FRUIT S:ATSTICS

PRUNLS, DRIED

Table 1

California Prunes: Bearing hareage, Production, Condition, Yield, and Farm Value 1919-1941

\begin{tabular}{|c|c|c|c|c|c|c|c|}
\hline \multirow[b]{2}{*}{$\begin{array}{l}\text { Crop } \\
\text { year }\end{array}$} & \multirow[b]{2}{*}{$\begin{array}{l}\text { Bearing } \\
\text { acreage }\end{array}$} & \multicolumn{2}{|c|}{ Production } & \multirow[b]{2}{*}{$\begin{array}{l}\text { Yield } \\
\text { per } \\
\text { bearing } \\
\text { acre }\end{array}$} & \multicolumn{2}{|c|}{ Farm* value of crop } & \multirow[b]{2}{*}{$\begin{array}{l}\text { Gross } \\
\text { income } \\
\text { per } \\
\text { bearing } \\
\text { acre }\end{array}$} \\
\hline & & Total & $\begin{array}{l}\text { oct. I } \\
\text { per cent } \\
\text { of full } \\
\text { crop }\end{array}$ & & $\begin{array}{l}\text { Price to } \\
\text { growers } \\
\text { per ton }\end{array}$ & $\begin{array}{l}\text { Total } \\
\text { returns } \\
\text { to } \\
\text { growrers }\end{array}$ & \\
\hline & 1 & 2 & 3 & 4 & 5 & 6 & 7 \\
\hline & $\begin{array}{l}1,000 \\
\text { acres }\end{array}$ & $\begin{array}{l}1,000 \text { tons, } \\
\text { dry weight }\end{array}$ & $\begin{array}{l}\text { Per } \\
\text { cent }\end{array}$ & $\begin{array}{l}\text { Short } \\
\text { tons, dry } \\
\text { weight }\end{array}$ & Dollars & $\begin{array}{l}1,000 \\
\text { dollars }\end{array}$ & Dollars \\
\hline 1919 & 104 & 140 & 105 & 1.35 & 240 & 33,600 & 323 \\
\hline 1920 & 105 & 98 & 73 & 0.93 & 170 & 16,660 & 159 \\
\hline 1921 & 106 & 100 & 76 & 0.94 & 14.5 & 14,500 & 137 \\
\hline 1922 & 111 & 126 & 80 & 1.14 & 150 & 18,900 & 170 \\
\hline 1923 & 119 & 114 & 62 & 0.96 & 100 & 11,400 & 96 \\
\hline 1924 & 129 & 139 & 65 & 1.08 & 110 & 15,290 & 119 \\
\hline 1925 & 139 & 146 & 65 & 1.05 & 120 & 17,520 & 126 \\
\hline 1926 & 156 & 151 & 64 & 0.97 & 100 & 15,100 & 97 \\
\hline 1927 & 162 & 225 & 82 & 1.39 & 75 & 16,875 & 104 \\
\hline 1928 & 168 & 221 & 75 & 1.32 & 100 & 22,100 & 132 \\
\hline 1929 & 171 & 103 & 37 & 0.60 & 160 & 16,480 & 96 \\
\hline 1930 & 171 & $274 t$ & 82 & 1.60 & 63 & 16,443 & 96 \\
\hline 1931 & 170 & 214 & 55 & 1.26 & 50 & 10,700 & 63 \\
\hline 1932 & 169 & $172 t$ & 53 & 1.02 & 55 & 9,240 & 55 \\
\hline 1933 & 166 & 182 & 58 & 1.10 & 80 & 14,560 & 88 \\
\hline 1934 & 166 & 171 & 54 & 1.03 & 60 & 10,260 & 62 \\
\hline 1935 & 157 & 258 & 77 & 1.64 & 58 & 14,964 & 95 \\
\hline 1936 & $155 \neq$ & 159 & 48 & 1.03 & 80 & 12,720 & 82 \\
\hline 1937 & $156^{\circ}$ & 249 & 74 & 1.60 & 54 & 13,446 & 86 \\
\hline 1938 & $154 \neq$ & $288 t$ & 87 & 1.87 & 42 & 9,408 & 61 \\
\hline 1939 & $151 \%$ & 185 & 57 & 1.23 & 67 & 12,395 & 82 \\
\hline 1940 & $146 \neq$ & $184 t$ & 62 & 1.26 & 55 & 9,625 & 66 \\
\hline $1941 \phi$ & 142 & $193 t$ & 69 & 1.36 & 69 & 12,558 & 88 \\
\hline
\end{tabular}

* Grovers' returns for naked fruit at growers' first delivery point.

$t$ Includes unharvested tonnage: $1930,13,000 ; 1932,4,000 ; 1938,64,000$;

$1940,9,000$; and 1941, 11,000.

FNonbearing acres: 1936, 9,964; 1937, 9,316; 1938, 8,881; $1939,8,312 ; 1940$, 7,450 .

$\$$ Data are preliminary and subject to revision.

Sources of data: Compiled by S. T. Shear, Giannini Foundation, largely based on reports of the California Crop Reporting Service, except some of the farm prices and production in 1922 and 1923 are estimates by S. W. Shear. 
$\therefore$

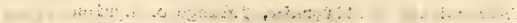

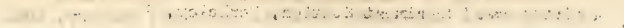

$\therefore \because \cdots+\cdots+\cdots+\cdots+\cdots$
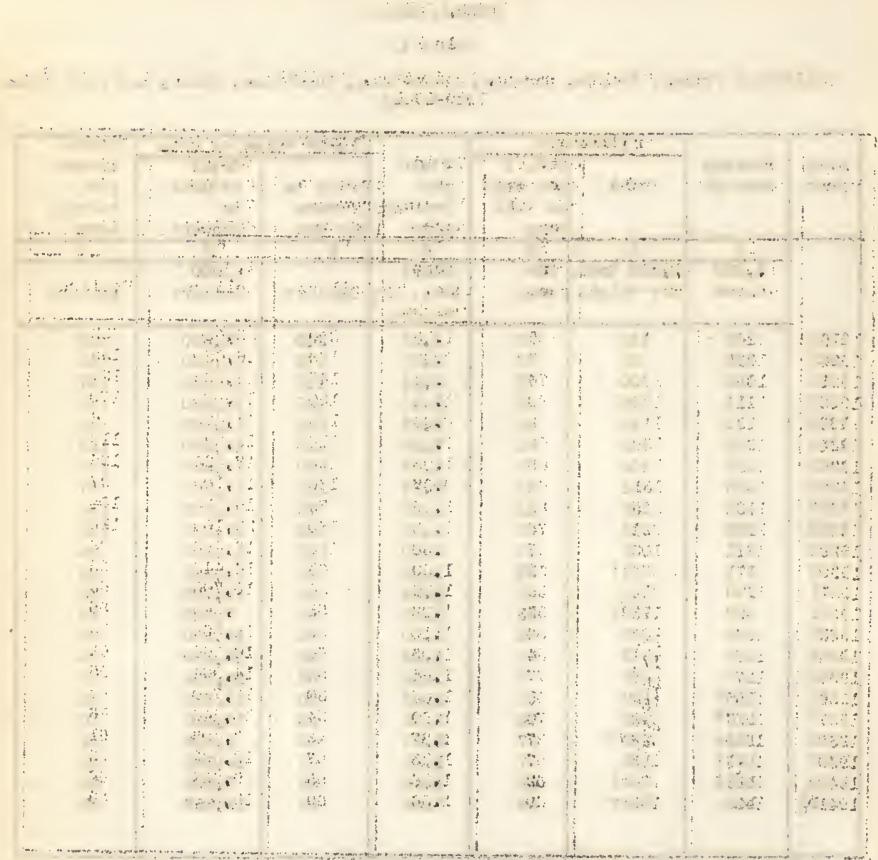

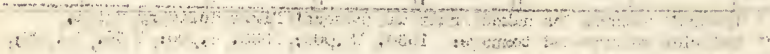

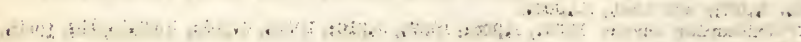

A.

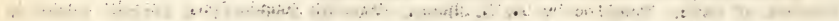

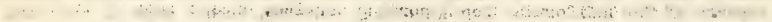

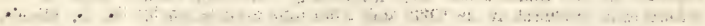


PRUNES, DRIED

January, 1942

Table 2

United States Production and Exports of Dried Prunes and california Prices, 1921-1941

\begin{tabular}{|c|c|c|c|c|c|c|c|c|}
\hline \multirow{4}{*}{$\begin{array}{l}\text { Crop } \\
\text { year }\end{array}$} & \multicolumn{5}{|c|}{ United States } & \multicolumn{3}{|c|}{ California } \\
\hline & \multirow{3}{*}{$\begin{array}{l}\text { Harvested } \\
\text { production }\end{array}$} & \multicolumn{4}{|c|}{ Exports } & \multirow{3}{*}{$\begin{array}{l}\text { Farm } \\
\text { prioe } \\
\text { per } \\
\text { pound }\end{array}$} & \multirow{3}{*}{$\begin{array}{l}\text { F.o.b. } \\
\text { packed } \\
\text { price } \\
\text { per } 1 \mathrm{~b} . \\
50 / 60 \text { 's }\end{array}$} & \multirow{3}{*}{$\begin{array}{l}\text { Wholesale } \\
\text { price per } \\
\text { lb. New } \\
\text { York City } \\
50 / 60 \text { 's }\end{array}$} \\
\hline & & \multicolumn{2}{|l|}{ Total } & \multirow{2}{*}{$\begin{array}{l}\text { As } \\
\text { Prunes }\end{array}$} & \multirow{2}{*}{$\begin{array}{l}\text { As } \\
\text { salad }\end{array}$} & & & \\
\hline & & $\begin{array}{c}\text { Per cent } \\
\text { of } \\
\text { production } \\
\end{array}$ & Quantity & & & & & \\
\hline \multirow{4}{*}{$\begin{array}{l}1921 \\
1922\end{array}$} & 1 & 2 & 3 & 4 & 5 & 6 & 7 & 8 \\
\hline & $\begin{array}{l}\text { Short tons } \\
\text { dry weight }\end{array}$ & Per cont & \multicolumn{3}{|c|}{$\begin{array}{c}\text { Short tons, unprocessed } \\
\text { dry weight }\end{array}$} & \multicolumn{3}{|c|}{ Cents } \\
\hline & 113,700 & $41+$ & $46,500+$ & 46,500 & $-t$ & 7.2 & 9.0 & 10.9 \\
\hline & 161,000 & $24 t$ & $39,100+$ & 39,100 & & 7.5 & 10.0 & 11.5 \\
\hline 1923 & 141,500 & & 74,300 & 72,300 & $2,000^{\prime}$ & 5.0 & 8.0 & $8 \cdot 6$ \\
\hline 1924 & 164,000 & 52 & 85,000 & 84,200 & 800 & 5.5 & 7.5 & 8.4 \\
\hline 1925 & 161,500 & 46 & 73,800 & 72,800 & 1,000 & 6.0 & 7.8 & 8.3 \\
\hline 1926 & 192,500 & 45 & 80,000 & 84,700 & 1,300 & $5 \cdot 0$ & 0.7 & $7 \cdot 4$ \\
\hline 1927 & 248,800 & 53 & 132,200 & 130,700 & 1,500 & 3.8 & $5 \cdot 6$ & 6.4 \\
\hline 1928 & 228,900 & 59 & 134,000 & 132,000 & 2,000 & $5 \cdot 0$ & 0.6 & 8.1 \\
\hline 1929 & 160,100 & 45 & 72,700 & 69,900 & 2,800 & 8.0 & $9 \cdot 6$ & 10.3 \\
\hline 1930 & 205,200 & 54 & 152,900 & 149,800 & 3,100 & 3.2 & 4.4 & 5.5 \\
\hline 1931 & 242,400 & 50 & 120,900 & 117,700 & 3,200 & 2.5 & 3.4 & 4.0 \\
\hline 1932 & 194,500 & 48 & 93,000 & 90,000 & 3,000 & 2.7 & 3.5 & $4 \cdot 7$ \\
\hline 1933 & 205,500 & 47 & 97,400 & 94,900 & 2,500 & 4.0 & $5 \cdot 7$ & 6.5 \\
\hline 1934 & 201,100 & 39 & 79,200 & 76,800 & 2,400 & 3.0 & 5.1 & 5.7 \\
\hline 1935 & 297,300 & 38 & 112,500 & 109,000 & 2,900 & 2.9 & 3.5 & 4.6 \\
\hline 1936 & 184,300 & 43 & 79,700 & 77,800 & 1,900 & 4.0 & 5.0 & 5.7 \\
\hline 1937 & 255,700 & 43 & $1 \cup 9,300$ & 106,400 & 2,900 & 2.7 & $3 \cdot 6$ & $4 \cdot 5$ \\
\hline 1938 & 238,300 & 44 & 105,000 & $1 \cup 1,500$ & 4,100 & 2.1 & 3.3 & $4 \cdot 6$ \\
\hline 1939 & 213,400 & 26 & 54,700 & 51,500 & 3,200 & 3.4 & 4.4 & 5.5 \\
\hline 1940 & 177,710 & 15 & 27,000 & 26,200 & 800 & 2.8 & 4.0 & 5.2 \\
\hline 1941キ & $188,410 \neq$ & & & & & $3.4 \neq$ & $5.8 \neq$ & $7.2 \neq$ \\
\hline
\end{tabular}

* Excludes Idaho production of almost zero to 900 tons a year.

+ Prune exports in salad excluded in 1921 and 1922 as data not available.

$\neq$ Preliminary estimates.

Sources of data: Compiled by S. W. Shear, Giannini Foucdation of Agricultural Economics.

Col. 1: Frcm reports of the United States and California Crop Reporting Service.

Cols. 3-5: Exports of dried prunes and 43 per cent of dried fruit salad exports years beginnine sept. 1. Based on U. S. Lionthly Sumary Forei $n$ Comeroe, except salad, 1923-1929, estimated largely on inspection data of Tried fruit Association of california.

Col. o: Estimates by $S$. W. Shear based partly on the California Crop Reports.

Col. 7: 1921-1931 based on packer reports to Giannini Foundation. 1932-1941 based on California Fruit lews quotations and monthly prune shipments.

Col. 3: Ionthly wholesale prices, reported currently in thew York Journal of Commerce, weighted by monthly California prune shipments. 

January, $1942 \quad 112$.

PRUNES, DRIED

Table 3

United States Exports of Dried Prunes* by Chief Countries of Destination, 1922-1940

\begin{tabular}{|c|c|c|c|c|c|c|c|c|c|}
\hline \multirow{2}{*}{\begin{tabular}{|c|} 
Year \\
beginning \\
September
\end{tabular}} & \multirow[b]{2}{*}{$\begin{array}{c}\text { Total } \\
\text { exports }\end{array}$} & \multicolumn{5}{|c|}{ Europe } & \multicolumn{3}{|c|}{ Other than Europe } \\
\hline & & Total & Germany & France & $\begin{array}{l}\text { United } \\
\text { Kingdom }\end{array}$ & $\begin{array}{l}\text { Other } \\
\text { Europe }\end{array}$ & Total & Canada & $\begin{array}{c}\text { Other } \\
\text { countries }\end{array}$ \\
\hline & \multicolumn{9}{|c|}{ Short tons, net declared dry woight $t$} \\
\hline Averages: & & & & & & & & & \\
\hline $1922-1926$ & 72,253 & 60,398 & 16,673 & 11,650 & 15,929 & 16,146 & 11,855 & 8,423 & 3,432 \\
\hline 1927-1931f & 122,517 & 107,500 & 36,312 & 19,224 & 19,754 & 32,210 & 15,017 & 9,074 & 5,943 \\
\hline $1929-1933 \neq$ & 106,699 & 93,507 & 30,066 & 17,378 & 17,606 & 28,457 & 13,192 & 8,041 & 5,151 \\
\hline $1934-1938 \neq$ & 97,012 & 80,009 & 7,321 & 20,445 & 19,386 & 32,857 & 17,003 & 8,869 & 8,134 \\
\hline \multicolumn{10}{|l|}{ Annual: } \\
\hline 1927 & 133,425 & 114,640 & 40,972 & 16,160 & 22,040 & 35,468 & 18,785 & 11,122 & 7,663 \\
\hline 1928 & 134,744 & 118,560 & 38,967 & 27,711 & 20,686 & 31,196 & 16,184 & 9,784 & 6,400 \\
\hline 1929 & 71,318 & 58,403 & 20,803 & 5,468 & 14,336 & 17,796 & 12,915 & 7,709 & 5,206 \\
\hline 1930 & 152,731 & 138,698 & 50,783 & 23,757 & 20,294 & 43,864 & 14,033 & 8,415 & 5,618 \\
\hline 1931 キ & 120,367 & 107,197 & 30,036 & 23,022 & 21,414 & 32,725 & 13,170 & 8,341 & 4,829 \\
\hline $1932 \neq$ & 92,208 & 79,311 & 18,352 & 21,550 & 16,097 & 23,312 & 12,897 & 8,062 & 4,835 \\
\hline $1933 \neq$ & 96,869 & 83,923 & 30,353 & 13,094 & 15,891 & 24,585 & 12,946 & 7,679 & 5,267 \\
\hline $1934 \mp$ & 78,923 & 63,765 & 5,148 & 14,006 & 16,671 & 27,940 & 15,158 & 9,113 & 6,045 \\
\hline $1935 \neq$ & 112,487 & 93,877 & 13,075 & 24,999 & 23,669 & 32,134 & 18,610 & 9,716 & 8,894 \\
\hline $1936 \neq$ & 79,970 & 63,559 & 4,812 & 20,826 & 15,150 & 22,771 & 16,411 & 8,920 & 7,491 \\
\hline $1937 \neq$ & 109,305 & 90,586 & 8,220 & 23,462 & 19,130 & 39,774 & 18,719 & 8,483 & 10,236 \\
\hline $1938 \neq$ & 104,377 & 88,256 & 5,350 & 18,931 & 22,311 & 41,664 & 16,121 & 8,115 & 8,006 \\
\hline 1939* & 53,020 & 37,020 & 4 & 1,663 & 16,364 & 18,989 & 16,000 & 8,774 & 7,226 \\
\hline 1940 q & $11,683^{4}$ & 1,929 & 0 & & & 1,921 & 9,754 & 5,901 & 3,853 \\
\hline & & & & & & & & & \\
\hline
\end{tabular}

* Do not include exports of dried prunes in dried fruit salad or compote.

tliet reight as shipped. Ilost prunes are exported processed, packed in boxes except to Germany which trkes most of them in bags in natural condition.

* Preliminary data as revised data by countries no longer published nonthly.

Seven nonths only, September 1940 - llarch 1941 as data by countries of destination not available for later months. Total exports for September - August were 27,014 tons.

Sources of data: Compiled by S. W. Shear, Giannini Foundation of igricultural Economics, University of California, from U. S. Dept. Com. Bur. For. and Dom. Com., llonthly Summary of Foreign Commerce and monthly mimeographed statements and U.S. Dept. Agr. Bur. Agr. Licon. (recently Agricultural Narketing Service) Federal-State llarket Hows Service. Prune Information Bulletins. (Himeo.) 

PRUNES, DRIED

Jañuary, 1942113.

Table 4. World Commercial Production of Dried Prunes by Countries Averages 1920-1938 and Annually 1924-1941

\begin{tabular}{|c|c|c|c|c|c|c|c|}
\hline \multirow[b]{2}{*}{$\begin{array}{l}\text { Crop } \\
\text { year }\end{array}$} & \multirow[b]{2}{*}{$\begin{array}{l}\text { World } \\
\text { total* }\end{array}$} & \multicolumn{3}{|c|}{ United States } & \multicolumn{3}{|c|}{ European } \\
\hline & & Total ${ }^{\dagger}$ & California & $\begin{array}{l}\text { Oregon and } \\
\text { Washington }\end{array}$ & Total ${ }^{\neq}$ & France & $\begin{array}{c}\text { Yugoslavia } \\
\text { (exports } \\
\text { only) }\end{array}$ \\
\hline \multirow{4}{*}{$\begin{array}{l}\text { Averages: } \\
1920-1923\end{array}$} & I & 2 & 3 & 4 & 5 & 6 & 7 \\
\hline & \multicolumn{7}{|c|}{ Short tons, dry weight } \\
\hline & & 133.300 & חח & & & & \\
\hline & & $\begin{array}{l}133,300 \\
199,100\end{array}$ & $\begin{array}{l}109,400 \\
176,400\end{array}$ & $\begin{array}{l}23,900 \\
22,700\end{array}$ & $\begin{array}{l}60,600 \\
37,300\end{array}$ & $\begin{array}{r}11,100 \\
5,300\end{array}$ & $\begin{array}{l}49,500 \\
32,000\end{array}$ \\
\hline $\begin{array}{l}1924-1928 \\
1929-1933\end{array}$ & $\begin{array}{l}236,400 \\
242,900\end{array}$ & 217,500 & 185,600 & 31,900 & 25,400 & $\begin{array}{l}5,500 \\
7,200\end{array}$ & $\begin{array}{l}32,000 \\
17,500\end{array}$ \\
\hline $\begin{array}{l}1929-1933 \\
1934-1938\end{array}$ & 267,500 & 235,300 & 212,200 & 23,100 & 32,200 & 4,900 & $\begin{array}{l}17,500 \\
16,900\end{array}$ \\
\hline \multicolumn{8}{|l|}{ Annual: } \\
\hline 1924 & 175,500 & 164,000 & 139,000 & 25,000 & 11,500 & 6,500 & 5,000 \\
\hline 1925 & 211,500 & 161,500 & 146,000 & 15,500 & 50,000 & 2,000 & 48,000 \\
\hline 1926 & 253,900 & 192,500 & 151,000 & 41,500 & 61,400 & 9,400 & 52,000 \\
\hline 1927 & 288,900 & 248,800 & 225,000 & 23,800 & 40,100 & 6,100 & 34,000 \\
\hline 1928 & 252,300 & 228,900 & 221,000 & 7,900 & 23,400 & 2,400 & 21,000 \\
\hline 1929 & 177,100 & 160,100 & 103,000 & 57,100 & 17,000 & 4,800 & 12,000 \\
\hline 1930 & 312,100 & 285,200 & $261,000+$ & 24,200 & 26,900 & $17,000^{\circ}$ & 9,500 \\
\hline 1931 & 256,300 & 242,400 & 214,000 & 28,400 & 13,900 & 4,000 & 9,500 \\
\hline 1932 & 228,100 & 194,500 & $168,000+$ & 26,500 & 33,600 & 2,500 & 30,000 \\
\hline 1933 & 240,800 & 205,500 & 182,000 & 23,500 & 35,300 & 7,500 & 26,500 \\
\hline 1934 & 235,900 & 201,100 & 171,000 & 30,100 & 34,800 & 6,000 & 24,100 \\
\hline 1935 & 330,300 & 297,300 & 258,000 & 39,300 & 33,000 & 5,000 & 11,100 \\
\hline 1936 & 239,600 & 184,300 & 159,000 & 25,300 & 55,300 & 8,000 & 36,300 \\
\hline 1937 & 261,900 & 255,700 & 249,000 & 6,700 & 6,200 & 1,000 & 3,200 \\
\hline 1938 & 269,900 & 238,300 & $224,000+$ & 14,300 & 31,600 & 4,500 & 9,900 \\
\hline 1939 & $275,500 q$ & 213,400 & 185,000 & 28,400 & 62,100 & 8,000 & 38,000 \\
\hline 1940 & & 177,710 & $175,000 t$ & 2,710 & II & H & II \\
\hline 1941$\}$ & & 188,410 & $182,000+$ & 6,410 & H & "I & " \\
\hline
\end{tabular}

* Vorld total excludes Australia and Union of South Africa which averaged

3,000 tons and 800 tons respeotively, 1934-1938.

+ Excludes quantities unharvested on account of market conditions. (See table 1 for California unharvested production. Oregon and Washington had unharvested tonnages neither dried nor used fresh every year since 1928 except 1936.)

F Total Europe is French production plus Yugoslavian exports and since 1929 also includes exports from Bulgaria and Rumania.

'́ Preliminary.

/l European exports not available for 1940 and 1941 but Bulgarian production in 1940 was 7,700 tons compared with 13,300 tons in 1939, and Yugoslavian production in 1940 was 3,300 tons compared with 80,300 tons in 1939 .

Sources of data: Compiled by S. W. Shear, Giannini Foundation.

$\mathrm{Col}$. 1: Sum of cols. 2 and 5.

Cols. 2-4: Estimates of U. S. Crop Reporting Board except California data, 1922 and 1923 are trade estimates.

Cols. 5-7: From reports of U. S. Dept. Agr., Foreign Agr. Service Division, released in Foreign. Prune Reports, Federal-State Market News Service. 


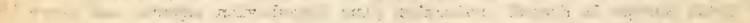

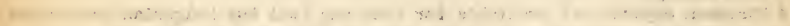

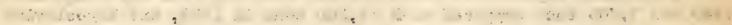

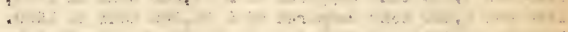

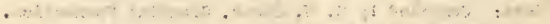

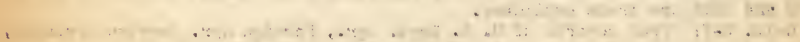

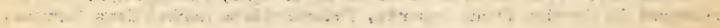

